\title{
RECENT RESULTS ON THE KOBAYASHI AND GREEN-GRIFFITHS-LANG CONJECTURES
}

\author{
JEAN-PIERRE DEMAILLY
}

\begin{abstract}
The study of entire holomorphic curves contained in projective algebraic varieties is intimately related to fascinating questions of geometry and number theory - especially through the concepts of curvature and positivity which are central themes in Kodaira's contributions to mathematics. The aim of these lectures is to present recent results concerning the geometric side of the problem. The Green-Griffiths-Lang conjecture stipulates that for every projective variety $X$ of general type over $\mathbb{C}$, there exists a proper algebraic subvariety $Y$ of $X$ containing all entire curves $f: \mathbb{C} \rightarrow X$. Using the formalism of directed varieties and jet bundles, we show that this assertion holds true in case $X$ satisfies a strong general type condition that is related to a certain jet-semi-stability property of the tangent bundle $T_{X}$. It is possible to exploit similar techniques to investigate a famous conjecture of Shoshichi Kobayashi (1970), according to which a generic algebraic hypersurface of dimension $n$ and of sufficiently large degree $d \geqslant d_{n}$ in the complex projective space $\mathbb{P}^{n+1}$ is hyperbolic: in the early 2000's, Yum-Tong Siu proposed a strategy that led in 2015 to a proof based on a clever use of slanted vector fields on jet spaces, combined with Nevanlinna theory arguments. In 2016, the conjecture has been settled in a different way by Damian Brotbek, making a more direct use of Wronskian differential operators and associated multiplier ideals; shortly afterwards, Ya Deng showed how the proof could be modified to yield an explicit value of $d_{n}$. We give here a short proof based on a substantial simplification of their ideas, producing a bound very similar to Deng's original estimate, namely $d_{n}=\left\lfloor\frac{1}{3}(e n)^{2 n+2}\right\rfloor$.
\end{abstract}

Key words: Kobayashi hyperbolic variety, directed manifold, genus of a curve, jet bundle, jet differential, jet metric, Chern connection and curvature, negativity of jet curvature, variety of general type, Kobayashi conjecture, Green-Griffiths conjecture, Lang conjecture.

MSC Classification (2010): 32H20, 32L10, 53C55, 14J40

Contribution to the $16^{\text {th }}$ Takagi lectures
in celebration of the $100^{\text {th }}$ anniversary of K. Kodaira's birth

CONTEnTs

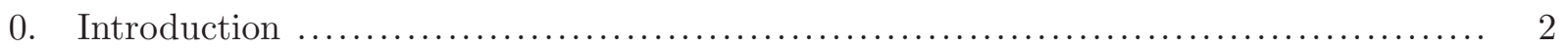

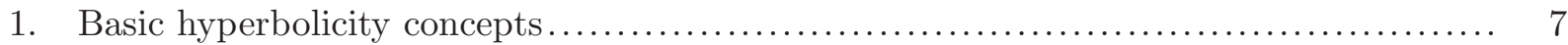

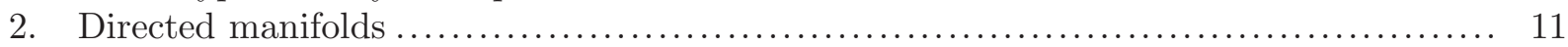

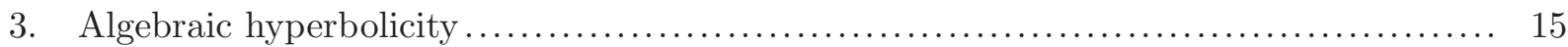

4. The Ahlfors-Schwarz lemma for metrics of negative curvature $\ldots \ldots \ldots \ldots \ldots \ldots \ldots \ldots \ldots . \ldots \ldots$

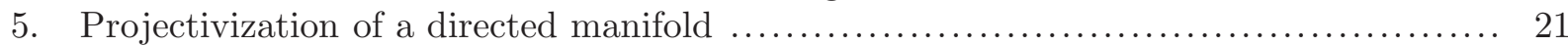

6. Jets of curves and Semple jet bundles ........................................... 23

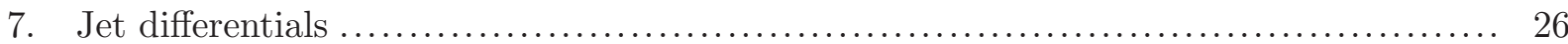

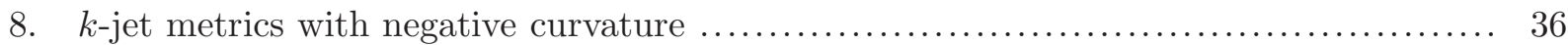

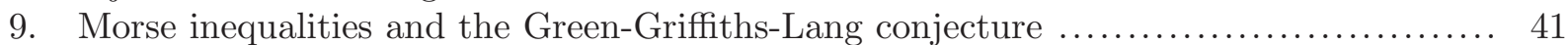

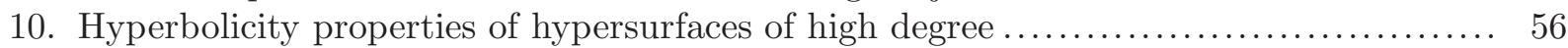

11. Strong general type condition and the GGL conjecture $\ldots \ldots \ldots \ldots \ldots \ldots \ldots \ldots \ldots \ldots \ldots \ldots \ldots$

12. Proof of the Kobayashi conjecture on generic hyperbolicity $\ldots \ldots \ldots \ldots \ldots \ldots \ldots \ldots \ldots . \ldots 3$

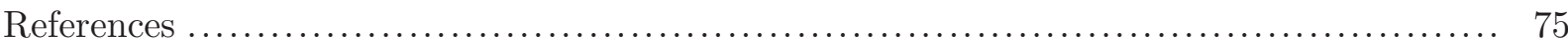

Expanded version of the Takagi lectures delivered at the University of Tokyo on November 6, 2015.

Work supported by the advanced ERC grant ALKAGE No. 670846 started in September 2015. 


\section{INTRODUCTION}

The goal of these lectures is to study the conjecture of Kobayashi [Kob70] on the hyperbolicity of generic hypersurfaces of high degree in projective space, and the related conjecture by GreenGriffiths [GrGr80] and Lang [Lang86] on the structure of entire curve loci.

Let us recall that a complex space $X$ is said to be hyperbolic in the sense of Kobayashi if analytic disks $f: \mathbb{D} \rightarrow X$ through a given point form a normal family. By a well known result of Brody [Bro78], a compact complex space is Kobayashi hyperbolic iff it does not contain any entire holomorphic curve $f: \mathbb{C} \rightarrow X$ ("Brody hyperbolicity").

In this paper entire holomorphic curves are assumed to be non-constant and simply called entire curves. If $X$ is not hyperbolic, a basic question is thus to analyze the geometry of entire holomorphic curves $f: \mathbb{C} \rightarrow X$, and especially to understand the entire curve locus of $X$, defined as the Zariski closure

$$
\operatorname{ECL}(X)={\overline{\bigcup_{f} f(\mathbb{C})}}^{\text {Zar }} .
$$

The Green-Griffiths-Lang conjecture, in its strong form, can be stated as follows.

0.2. GGL conjecture. Let $X$ be a projective variety of general type. Then $Y=\operatorname{ECL}(X)$ is a proper algebraic subvariety $Y \subsetneq X$.

Equivalently, there exists $Y \subsetneq X$ such that every entire curve $f: \mathbb{C} \rightarrow X$ satisfies $f(\mathbb{C}) \subset Y$. A weaker form of the GGL conjecture states that entire curves are algebraically degenerate, i.e. that $f(\mathbb{C}) \subset Y_{f} \subsetneq X$ where $Y_{f}$ may depend on $f$.

If $X \subset \mathbb{P}_{\mathbb{C}}^{N}$ is defined over a number field $\mathbb{K}_{0}$ (i.e. by polynomial equations with coefficients in $\mathbb{K}_{0}$ ), one defines the Mordell locus, denoted $\operatorname{Mordell}(X)$, to be the smallest complex subvariety $Y$ in $X$ such that the set of $\mathbb{K}$-points $X(\mathbb{K}) \backslash Y$ is finite for every number field $\mathbb{K} \supset \mathbb{K}_{0}$. Lang [Lang86] conjectured that one should always have $\operatorname{Mordell}(X)=\operatorname{ECL}(X)$ in this situation. This conjectural arithmetical statement would be a vast generalization of the Mordell-Faltings theorem, and is one of the strong motivations to study the geometric GGL conjecture as a first step. S. Kobayashi [Kob70] had earlier made the following tantalizing conjecture.

0.3. Conjecture (Kobayashi).

(a) $A$ (very) generic hypersurface $X \subset \mathbb{P}^{n+1}$ of degree $d \geqslant d_{n}$ large enough is hyperbolic, especially it does not possess any entire holomorphic curve $f: \mathbb{C} \rightarrow X$.

(b) The complement $\mathbb{P}^{n} \backslash H$ of a (very) generic hypersurface $H \subset \mathbb{P}^{n}$ of degree $d \geqslant d_{n}^{\prime}$ large enough is hyperbolic.

It should be noticed that the existence of a smooth hyperbolic hypersurface $X \subset \mathbb{P}^{n+1}$ in 0.3 (a), or a hyperbolic complement $\mathbb{P}^{n} \backslash H$ with $H$ smooth irreducible in $0.3(\mathrm{~b})$, is already a hard problem; many efforts were initially concentrated on this problem. As Zaidenberg observed, a smooth deformation of a union of $2 n+1$ hyperplanes in $\mathbb{P}^{n}$ is not necessarily Kobayashi hyperbolic, and the issue is non-trivial at all. The existence problem was initially solved for sufficiently high degree hypersurfaces through a number of examples:

- case (a) for $n=2$ and degree $d \geqslant 50$ by BrodyGreen [BrGr77];

- case (b) for $n=2$ by [AzSu80] (as a consequence of [BrGr77]);

- cases (a) and (b) for $n \geqslant 3$ by MasudaNoguchi [MaNo96].

Improvements in the degree estimates were later obtained in [Shi98], [Fuj01], [ShZa02], in addition to many other papers dealing with low dimensional varieties $(n=2,3)$.

We now describe a number of known results concerning the question of generic hyperbolicity, according to the Kobayashi conjectures $0.3(\mathrm{a}, \mathrm{b})$. M. Zaidenberg observed in [Zai87] that the complement of a general hypersurface of degree $2 n$ in $\mathbb{P}^{n}$ is not hyperbolic; as a consequence, one must take $d_{n}^{\prime} \geqslant 2 n+1$ in 0.3 (b). This observation, along with Fujimoto's classical result that 
the complement of $2 n+1$ hyperplanes of $\mathbb{P}^{n}$ in general position is hyperbolic and hyperbolically embedded in $\mathbb{P}^{n}$ ([Fuj72]) led Zaidenberg to propose the bound $d_{n}^{\prime}=2 n+1$ for $n \geqslant 1$. Another famous result due to Clemens [Cle86], Ein [Ein88, Ein91] and Voisin [Voi96], states that every subvariety $Y$ of a generic algebraic hypersurface $X \subset \mathbb{P}^{n+1}$ of degree $d \geqslant 2 n+1$ is of general type for $n \geqslant 2$ (for surfaces $X \subset \mathbb{P}^{3}$, Geng Xu [Xu94] also obtained some refined information for the genera of algebraic curves drawn in $X$ ). The bound was subsequently improved to $d \geqslant d_{n}=2 n$ for $n \geqslant 5$ by Pacienza [Pac04]. That the same bound $d_{n}$ holds for Kobayashi hyperbolicity would then be a consequence of the Green-Griffiths-Lang conjecture. By these results, one can hope in the compact case that the optimal bound $d_{n}$ is $d_{1}=4, d_{n}=2 n+1$ for $n=2,3,4$ and $d_{n}=2 n$ for $n \geqslant 5$. The case of complements $\mathbb{P}^{n} \backslash H$ (the so-called "logarithmic case") is a priori somewhat easier to deal with: in fact, on can then exploit the fact that the hyperbolicity of the hypersurface $X=\left\{w^{d}=P(z)\right\} \subset \mathbb{P}^{n+1}$ implies the hyperbolicity of the complement $\mathbb{P}^{n} \backslash H$, when $H=\{P(z)=0\}$. Pacienza and Rousseau [PaRo07] proved that for $H$ very general of degree $d \geqslant 2 n+2-k$, any $k$-dimensional $\log$-subvariety $(Y, D)$ of $\left(\mathbb{P}^{n}, H\right)$ is of log-general type, i.e. any log-resolution $\mu: \tilde{Y} \rightarrow Y$ of $(Y, D)$ has a big log-canonical bundle $K_{\widetilde{Y}}\left(\mu^{*} D\right)$.

One of the early important result in the direction of Conjecture 0.2 is the proof of the Bloch conjecture, as proposed by Bloch [Blo26a] and Ochiai [Och77]: this is the special case of the conjecture when the irregularity of $X$ satisfies $q=h^{0}\left(X, \Omega_{X}^{1}\right)>\operatorname{dim} X$. Various solutions have then been obtained in fundamental papers of Noguchi [Nog77a, Nog81a, Nog81b], Kawamata [Kaw80], Green-Griffiths [GrGr80], McQuillan [McQ96], and the book of Noguchi-Winkelmann [NoWi13], by means of different techniques. Especially, assuming $\mathrm{X}$ to be of (log-) general type, it is now known by [NWY07, NWY13] and [LuWi12] that if the (log-) irregularity is $q \geqslant \operatorname{dim} X$, then no entire curve $f: \mathbb{C} \rightarrow X$ has a Zariski dense image, and the GGL conjecture holds in the compact (i.e. non logarithmic) case. In the case of complex surfaces, major progress was achieved by Lu, Miyaoka and Yau [LuYa90], [LuMi95, 96], [Lu96]; McQuillan [McQ96] extended these results to the case of all surfaces satisfying $c_{1}^{2}>c_{2}$, in a situation where there are many symmetric differentials, e.g. sections of $H^{0}\left(X, S^{m} T_{X}^{*} \otimes \mathcal{O}(-1)\right), m \gg 1$ (cf. also [McQ99], [DeEG00] for applications to hyperbolicity). A more recent result is the deep statement due to Diverio, Merker and Rousseau [DMR10], confirming Conjecture 0.2 when $X \subset \mathbb{P}^{n+1}$ is a generic non-singular hypersurface of sufficiently large degree $d \geqslant 2^{n^{5}}$ (cf. $\S 10$ ); in the case $n=2$ of surfaces in $\mathbb{P}^{3}$, we are here in the more difficult situation where symmetric differentials do not exist (we have $c_{1}^{2}<c_{2}$ in this case). Conjecture 0.2 was also considered by S. Lang [Lang86, Lang87] in view of arithmetic counterparts of the above geometric statements.

Although these optimal conjectures are still unsolved at present, substantial progress was achieved in the meantime, for a large part via the technique of producing jet differentials. This is done either by direct calculations or by various indirect methods: Riemann-Roch calculations, vanishing theorems ... Vojta [Voj87] and McQuillan [McQ98] introduced the "diophantine approximation" method, which was soon recognized to be an important tool in the study of holomorphic foliations, in parallel with Nevanlinna theory and the construction of Ahlfors currents. Around 2000, Siu [Siu02, 04] showed that generic hyperbolicity results in the direction of the Kobayashi conjecture could be investigated by combining the algebraic techniques of Clemens, Ein and Voisin with the existence of certain "vertical" meromorphic vector fields on the jet space of the universal hypersurface of high degree; these vector fields are actually used to differentiate the global sections of the jet bundles involved, so as to produce new sections with a better control on the base locus. Also, during the years 2007-2010, it was realized [Dem07a, 07b, Dem11] that holomorphic Morse inequalities could be used to prove the existence of jet differentials; in 2010, Diverio, Merker and Rousseau [DMR10] were able in that way to prove the Green-Griffiths conjecture for generic hypersurfaces of high degree in projective space, e.g. for $d \geqslant 2^{n^{5}}$ - their proof makes an essential use of Siu's differentiation technique via meromorphic vector fields, as improved by Păun [Pau08] and Merker [Mer09] in 2008. The present study will be focused on the holomorphic Morse inequality technique; 
as an application, a partial answer to the Kobayashi and Green-Griffiths-Lang conjecture can be obtained in a very wide context : the basic general result achieved in [Dem11] consists of showing that for every projective variety of general type $X$, there exists a global algebraic differential operator $P$ on $X$ (in fact many such operators $P_{j}$ ) such that every entire curve $f: \mathbb{C} \rightarrow X$ must satisfy the differential equations $P_{j}\left(f ; f^{\prime}, \ldots, f^{(k)}\right)=0$. One also recovers from there the result of Diverio-Merker-Rousseau on the generic Green-Griffiths conjecture (with an even better bound asymptotically as the dimension tends to infinity), as well as a result of Diverio-Trapani [DT10] on the hyperbolicity of generic 3-dimensional hypersurfaces in $\mathbb{P}^{4}$. Siu [Siu04, Siu15] has introduced a more explicit but more computationally involved approach based on the use of "slanted vector fields" on jet spaces, extending ideas of Clemens [Cle86] and Voisin [Voi96] (cf. section 10 for details); [Siu15] explains how this strategy can be used to assert the Kobayashi conjecture for $d \geqslant d_{n}$, with a very large bound and non-effective bound $d_{n}$ instead of $2 n+1$.

As we will see here, it is useful to work in a more general context and to consider the category of directed varieties. When the problems under consideration are birationally invariant, as is the case of the Green-Griffiths-Lang conjecture, varieties can be replaced by non singular models; for this reason, we will mostly restrict ourselves to the case of non-singular varieties in the rest of the introduction. A directed projective manifold is a pair $(X, V)$ where $X$ is a projective manifold equipped with an analytic linear subspace $V \subset T_{X}$, i.e. a closed irreducible complex analytic subset $V$ of the total space of $T_{X}$, such that each fiber $V_{x}=V \cap T_{X, x}$ is a complex vector space. If $X$ is not connected, $V$ should rather be assumed to be irreducible merely over each connected component of $X$, but we will hereafter assume that our manifolds are connected. A morphism $\Phi:(X, V) \rightarrow(Y, W)$ in the category of directed manifolds is an analytic map $\Phi: X \rightarrow Y$ such that $\Phi_{*} V \subset W$. We refer to the case $V=T_{X}$ as being the absolute case, and to the case $V=T_{X / S}=\operatorname{Ker} d \pi$ for a fibration $\pi: X \rightarrow S$, as being the relative case; $V$ may also be taken to be the tangent space to the leaves of a singular analytic foliation on $X$, or maybe even a non-integrable linear subspace of $T_{X}$. We are especially interested in entire curves that are tangent to $V$, namely non-constant holomorphic morphisms $f:\left(\mathbb{C}, T_{\mathbb{C}}\right) \rightarrow(X, V)$ of directed manifolds. In the absolute case, these are just arbitrary entire curves $f: \mathbb{C} \rightarrow X$.

0.4. Generalized GGL conjecture. Let $(X, V)$ be a projective directed manifold. Define the entire curve locus of $(X, V)$ to be the Zariski closure of the locus of entire curves tangent to $V$, i.e.

$$
\operatorname{ECL}(X, V)={\overline{\bigcup_{f:\left(\mathbb{C}, T_{\mathbb{C}}\right) \rightarrow(X, V)} f(\mathbb{C})}}^{\text {Zar }} .
$$

Then, if $(X, V)$ is of general type in the sense that the canonical sheaf sequence $K_{V}^{\bullet}$ is big (cf. Prop 2.11 below), $Y=\operatorname{ECL}(X, V)$ is a proper algebraic subvariety $Y \subsetneq X$.

[We will say that $(X, V)$ is Brody hyperbolic if $\operatorname{ECL}(X, V)=\emptyset$; by Brody's reparametrization technique, this is equivalent to Kobayashi hyperbolicity whenever $X$ is compact.]

In case $V$ has no singularities, the canonical sheaf $K_{V}$ is defined to be $(\operatorname{det}(\mathcal{O}(V)))^{*}$ where $\mathcal{O}(V)$ is the sheaf of holomorphic sections of $V$, but in general this naive definition would not work. Take for instance a generic pencil of elliptic curves $\lambda P(z)+\mu Q(z)=0$ of degree 3 in $\mathbb{P}_{\mathbb{C}}^{2}$, and the linear space $V$ consisting of the tangents to the fibers of the rational map $\mathbb{P}_{\mathbb{C}}^{2}-\rightarrow \mathbb{P}_{\mathbb{C}}^{1}$ defined by $z \mapsto Q(z) / P(z)$. Then $V$ is given by

$$
0 \longrightarrow \mathcal{O}(V) \longrightarrow \mathcal{O}\left(T_{\mathbb{P}_{\mathbb{C}}^{2}} \stackrel{P d Q-Q d P}{\longrightarrow} \mathcal{O}_{\mathbb{P}_{\mathbb{C}}^{2}}(6) \otimes \mathcal{J}_{S} \longrightarrow 0\right.
$$

where $S=\operatorname{Sing}(V)$ consists of the 9 points $\{P(z)=0\} \cap\{Q(z)=0\}$, and $\mathcal{J}_{S}$ is the corresponding ideal sheaf of $S$. Since $\operatorname{det}\left(\mathcal{O}\left(T_{\mathbb{P}^{2}}\right)\right)=\mathcal{O}(3)$, we see that $\left(\operatorname{det}(\mathcal{O}(V))^{*}=\mathcal{O}(3)\right.$ is ample, thus the generalized GGL conjecture 0.4 would not have a positive answer (all leaves are elliptic or singular rational curves and thus covered by entire curves). An even more "degenerate" example is obtained with a generic pencil of conics, in which case $\left(\operatorname{det}(\mathcal{O}(V))^{*}=\mathcal{O}(1)\right.$ and $\# S=4$. 
If we want to get a positive answer to the generalized GGL conjecture 0.4, it is therefore indispensable to give a definition of $K_{V}$ that incorporates in a suitable way the singularities of $V$; this will be done in Def. 2.12 (see also Prop. 2.11). The goal is then to give a positive answer to the conjecture under some possibly more restrictive conditions for the pair $(X, V)$. These conditions will be expressed in terms of the tower of Semple jet bundles

$$
\left(X_{k}, V_{k}\right) \rightarrow\left(X_{k-1}, V_{k-1}\right) \rightarrow \ldots \rightarrow\left(X_{1}, V_{1}\right) \rightarrow\left(X_{0}, V_{0}\right):=(X, V)
$$

which we define more precisely in Section 1, following [Dem95]. It is constructed inductively by setting $X_{k}=P\left(V_{k-1}\right)$ (projective bundle of lines of $V_{k-1}$ ), and all $V_{k}$ have the same rank $r=\operatorname{rank} V$, so that $\operatorname{dim} X_{k}=n+k(r-1)$ where $n=\operatorname{dim} X$. Entire curve loci have their counterparts for all stages of the Semple tower, namely, one can define

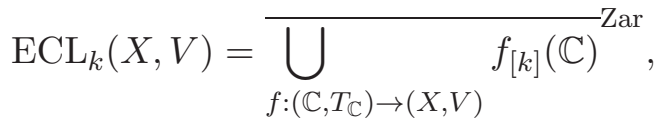

where $f_{[k]}:\left(\mathbb{C}, T_{\mathbb{C}}\right) \rightarrow\left(X_{k}, V_{k}\right)$ is the $k$-jet of $f$. These are by definition algebraic subvarieties of $X_{k}$, and if we denote by $\pi_{k, \ell}: X_{k} \rightarrow X_{\ell}$ the natural projection from $X_{k}$ to $X_{\ell}, 0 \leqslant \ell \leqslant k$, we get immediately

$$
\pi_{k, \ell}\left(\operatorname{ECL}_{k}(X, V)\right)=\operatorname{ECL}_{\ell}(X, V), \quad \operatorname{ECL}_{0}(X, V)=\operatorname{ECL}(X, V) .
$$

Let $\mathcal{O}_{X_{k}}(1)$ be the tautological line bundle over $X_{k}$ associated with the projective structure. We define the $k$-stage Green-Griffiths locus of $(X, V)$ to be

$$
\operatorname{GG}_{k}(X, V)=\overline{\left(X_{k} \backslash \Delta_{k}\right) \cap \bigcap_{m \in \mathbb{N}}\left(\text { base locus of } \mathcal{O}_{X_{k}}(m) \otimes \pi_{k, 0}^{*} A^{-1}\right)}
$$

where $A$ is any ample line bundle on $X$ and $\Delta_{k}=\bigcup_{2 \leqslant \ell \leqslant k} \pi_{k, \ell}^{-1}\left(D_{\ell}\right)$ is the union of "vertical divisors" (see (6.9) and (7.17); the vertical divisors play no role and have to be removed in this context; for this, one uses the fact that $f_{[k]}(\mathbb{C})$ is not contained in any component of $\Delta_{k}$, cf. [Dem95]). Clearly, $\mathrm{GG}_{k}(X, V)$ does not depend on the choice of $A$.

0.9. Basic vanishing theorem for entire curves. Let $(X, V)$ be an arbitrary directed variety with $X$ non-singular, and let $A$ be an ample line bundle on $X$. Then any entire curve $f:\left(\mathbb{C}, T_{\mathbb{C}}\right) \rightarrow(X, V)$ satisfies the differential equations $P\left(f ; f^{\prime}, \ldots, f^{(k)}\right)=0$ arising from sections $\sigma \in H^{0}\left(X_{k}, \mathcal{O}_{X_{k}}(m) \otimes \pi_{k, 0}^{*} A^{-1}\right)$. As a consequence, one has

$$
\mathrm{ECL}_{k}(X, V) \subset \mathrm{GG}_{k}(X, V) .
$$

The main argument goes back to [GrGr80]. We will give here a complete proof of Theorem 0.9, based only on the arguments [Dem95], namely on the Ahlfors-Schwarz lemma (the alternative proof given in [SiYe96b] uses Nevanlinna theory and is analytically more involved). By (0.7) and Theorem 0.9 we infer that

$$
\operatorname{ECL}(X, V) \subset \mathrm{GG}(X, V),
$$

where $\mathrm{GG}(X, V)$ is the global Green-Griffiths locus of $(X, V)$ defined by

$$
\mathrm{GG}(X, V)=\bigcap_{k \in \mathbb{N}} \pi_{k, 0}\left(\mathrm{GG}_{k}(X, V)\right) .
$$

The main result of [Dem11] (Theorem 2.37 and Cor. 3.4) implies the following useful information: 0.12. Theorem. Assume that $(X, V)$ is of "general type", i.e. that the pluricanonical sheaf sequence $K_{V}^{\bullet}$ is big on $X$. Then there exists an integer $k_{0}$ such that $\mathrm{GG}_{k}(X, V)$ is a proper algebraic subset of $X_{k}$ for $k \geqslant k_{0}\left[\right.$ though $\pi_{k, 0}\left(\mathrm{GG}_{k}(X, V)\right)$ might still be equal to $X$ for all $\left.k\right]$. 
In fact, if $F$ is an invertible sheaf on $X$ such that $K_{V}^{\bullet} \otimes F$ is big (cf. Prop. 2.11), the probabilistic estimates of [Dem11, Cor. 2.38 and Cor. 3.4] produce global sections of

$$
\mathcal{O}_{X_{k}}(m) \otimes \pi_{k, 0}^{*} \mathcal{O}\left(\frac{m}{k r}\left(1+\frac{1}{2}+\ldots+\frac{1}{k}\right) F\right)
$$

for $m \gg k \gg 1$. The (long and elaborate) proof uses a curvature computation and singular holomorphic Morse inequalities to show that the line bundles involved in (0.11) are big on $X_{k}$ for $k \gg 1$. One applies this to $F=A^{-1}$ with $A$ ample on $X$ to produce sections and conclude that $\mathrm{GG}_{k}(X, V) \subsetneq X_{k}$.

Thanks to (0.10), the GGL conjecture is satisfied whenever $\operatorname{GG}(X, V) \subsetneq X$. By [DMR10], this happens for instance in the absolute case when $X$ is a generic hypersurface of degree $d \geqslant 2^{n^{5}}$ in $\mathbb{P}^{n+1}$ (see also [Pau08] for better bounds in low dimensions, and [Siu02, Siu04]). However, as already mentioned in [Lang86], very simple examples show that one can have $\mathrm{GG}(X, V)=X$ even when $(X, V)$ is of general type, and this already occurs in the absolute case as soon as $\operatorname{dim} X \geqslant 2$. A typical example is a product of directed manifolds

$$
(X, V)=\left(X^{\prime}, V^{\prime}\right) \times\left(X^{\prime \prime}, V^{\prime \prime}\right), \quad V=\operatorname{pr}^{\prime *} V^{\prime} \oplus \operatorname{pr}^{\prime \prime *} V^{\prime \prime} .
$$

The absolute case $V=T_{X}, V^{\prime}=T_{X^{\prime}}, V^{\prime \prime}=T_{X^{\prime \prime}}$ on a product of curves is the simplest instance. It is then easy to check that $\operatorname{GG}(X, V)=X$, cf. (3.2). Diverio and Rousseau [DR15] have given many more such examples, including the case of indecomposable varieties $\left(X, T_{X}\right)$, e.g. Hilbert modular surfaces, or more generally compact quotients of bounded symmetric domains of rank $\geqslant 2$.

The problem here is the failure of some sort of stability condition that is introduced in Remark 11.10. This leads us to make the assumption that the directed pair $(X, V)$ is strongly of general type: by this, we mean that the induced directed structure $(Z, W)$ on each non-vertical subvariety $Z \subset X_{k}$ that projects onto $X$ either has $\operatorname{rank} W=0$ or is of general type modulo $X_{\bullet} \rightarrow X$, in the sense that $K_{W_{\ell}}^{\bullet} \otimes \mathcal{O}_{Z_{\ell}}(p)_{\mid Z_{\ell}}$ is big for some stage of the Semple tower of $(Z, W)$ and some $p \geqslant 0$ (see Section 11 for details - one may have to replace $Z_{\ell}$ by a suitable modification). Our main result can be stated as follows:

0.15. Theorem (partial solution to the generalized GGL conjecture). Let $(X, V)$ be a directed pair that is strongly of general type. Then the Green-Griffiths-Lang conjecture holds true for $(X, V)$, namely $\operatorname{ECL}(X, V)$ is a proper algebraic subvariety of $X$.

The proof proceeds through a complicated induction on $n=\operatorname{dim} X$ and $k=\operatorname{rank} V$, which is the main reason why we have to introduce directed varieties, even in the absolute case. An interesting feature of this result is that the conclusion on $\operatorname{ECL}(X, V)$ is reached without having to know anything about the Green-Griffiths locus $\operatorname{GG}(X, V)$, even a posteriori. Nevertheless, this is not yet enough to confirm the GGL conjecture. Our hope is that pairs $(X, V)$ that are of general type without being strongly of general type - and thus exhibit some sort of "jet-instability" - can be investigated by different methods, e.g. by the diophantine approximation techniques of McQuillan [McQ98]. However, Theorem 0.15 provides a sufficient criterion for Kobayashi hyperbolicity [Kob70], thanks to the following concept of algebraic jet-hyperbolicity.

0.16. Definition. A directed variety $(X, V)$ will be said to be algebraically jet-hyperbolic if the induced directed variety structure $(Z, W)$ on every non-vertical irreducible algebraic variety $Z$ of $X_{k}$ with $\operatorname{rank} W \geqslant 1$ is such that $K_{W_{\ell}}^{\bullet} \otimes \mathcal{O}_{Z_{\ell}}(p)_{\mid Z_{\ell}}$ is big for some stage of the Semple tower of $(Z, W)$ and some $p \geqslant 0$ [possibly after taking a suitable modification of $Z_{\ell}$; see Sections 11,12 for the definition of induced directed structures and further details]. We also say that a projective manifold $X$ is algebraically jet-hyperbolic if $\left(X, T_{X}\right)$ is.

In this context, Theorem 0.15 yields the following connection between algebraic jet-hyperbolicity and the analytic concept of Kobayashi hyperbolicity.

0.17. Theorem. Let $(X, V)$ be a directed variety structure on a projective manifold $X$. Assume that $(X, V)$ is algebraically jet-hyperbolic. Then $(X, V)$ is Kobayashi hyperbolic. 
The following conjecture would then make a bridge between these theorems and the GGL and Kobayashi conjectures.

0.18. Conjecture. Let $X \subset \mathbb{P}^{n+c}$ be a complete intersection of hypersurfaces of respective degrees $d_{1}, \ldots, d_{c}, \operatorname{codim} X=c$.

(a) If $X$ is non-singular and of general type, i.e. if $\sum d_{j} \geqslant n+c+2$, then $X$ is in fact strongly of general type.

(b) If $X$ is (very) generic and $\sum d_{j} \geqslant 2 n+c$, then $X$ is algebraically jet-hyperbolic.

Since Conjecture 0.18 only deals with algebraic statements, our hope is that a proof can be obtained through a suitable deepening of the techniques introduced by Clemens, Ein, Voisin and Siu. Under the slightly stronger condition $\sum d_{j} \geqslant 2 n+c+1$, Voisin showed indeed that every subvariety $Y \subset X$ is of general type, if $X$ is generic. To prove the Kobayashi conjecture in its optimal incarnation, we would need to show that such $Y$ 's are strongly of general type.

In the direction of getting examples of low degrees, Dinh Tuan Huynh [DTH16a] showed that there are families of hyperbolic hypersurfaces of degree $2 n+2$ in $\mathbb{P}^{n+1}$ for $2 \leqslant n \leqslant 5$, and in [DTH16b] he showed that certain small deformations (in Euclidean topology) of a union of $\left\lceil(n+3)^{2} / 4\right\rceil$ hyperplanes in general position in $\mathbb{P}^{n+1}$ are hyperbolic. In [Ber18], G. Bérczi stated a positivity conjecture for Thom polynomials of Morin singularities (see also [BeSz12]), and announced that it would imply a polynomial bound $d_{n}=2 n^{9}+1$ for the generic hyperbolicity of hypersurfaces. By using the "technology" of Semple towers and following new ideas introduced by D. Brotbek [Brot17] and Ya Deng [Deng16], we prove here the following effective (although nonoptimal) version of the Kobayashi conjecture on generic hyperbolicity.

0.19. Theorem. Let $Z$ be a projective $(n+1)$-dimensional manifold and $A$ a very ample line bundle on $Z$. Then, for a general section $\sigma \in H^{0}\left(Z, A^{d}\right)$ and $d \geqslant d_{n}$, the hypersurface $X_{\sigma}=\sigma^{-1}(0)$ is Kobayashi hyperbolic and, in fact, satisfies the stronger property of being algebraically jet hyperbolic. The bound $d_{n}$ for the degree can be taken to be $d_{n}:=\left\lfloor\frac{1}{3}(e n)^{2 n+2}\right\rfloor$.

I would like to thank Damian Brotbek, Ya Deng, Simone Diverio, Gianluca Pacienza, Erwan Rousseau, Mihai Păun and Mikhail Zaidenberg for very stimulating discussions on these questions. These notes also owe a lot to their work. I also with to thank the unknown referees for a large number of corrections and very useful suggestions.

\section{BASIC HYPERBOLICITY CONCEPTS}

\section{A. KoBAyASHi HYPERBOLICITY}

We first recall a few basic facts concerning the concept of hyperbolicity, according to S. Kobayashi [Kob70, Kob76, Kob98]. Let $X$ be a complex space. Given two points $p, q \in X$, let us consider a chain of analytic disks from $p$ to $q$, that is a sequence of holomorphic maps $f_{0}, f_{1}, \ldots, f_{k}: \Delta \rightarrow X$ from the unit disk $\Delta=D(0,1) \subset \mathbb{C}$ to $X$, together with pairs of points $a_{0}, b_{0}, \ldots, a_{k}, b_{k}$ of $\Delta$ such that

$$
p=f_{0}\left(a_{0}\right), \quad q=f_{k}\left(b_{k}\right), \quad f_{i}\left(b_{i}\right)=f_{i+1}\left(a_{i+1}\right), \quad i=0, \ldots, k-1 .
$$

Denoting this chain by $\alpha$, we define its length $\ell(\alpha)$ to be

$$
\ell(\alpha)=d_{P}\left(a_{1}, b_{1}\right)+\cdots+d_{P}\left(a_{k}, b_{k}\right)
$$

where $d_{P}$ is the Poincaré distance on $\Delta$, and the Kobayashi pseudodistance $d_{X}^{K}$ on $X$ to be

$$
d_{X}^{K}(p, q)=\inf _{\alpha} \ell(\alpha) .
$$

A Finsler metric (resp. pseudometric) on a vector bundle $E$ is a homogeneous positive (resp. nonnegative) function $N$ on the total space $E$, that is,

$$
N(\lambda \xi)=|\lambda| N(\xi) \quad \text { for all } \lambda \in \mathbb{C} \text { and } \xi \in E,
$$


but in general $N$ is not assumed to be subadditive (i.e. convex) on the fibers of $E$. A Finsler (pseudo-)metric on $E$ is thus nothing but a Hermitian (semi-)norm on the tautological line bundle $\mathcal{O}_{P(E)}(-1)$ of lines of $E$ over the projectivized bundle $Y=P(E)$. The Kobayashi-Royden infinitesimal pseudometric on $X$ is the Finsler pseudometric on the tangent bundle $T_{X}$ defined by

$$
\mathbf{k}_{X}(\xi)=\inf \left\{\lambda>0 ; \exists f: \Delta \rightarrow X, f(0)=x, \lambda f^{\prime}(0)=\xi\right\}, \quad x \in X, \xi \in T_{X, x} .
$$

Here, if $X$ is not smooth at $x$, we take $T_{X, x}=\left(\mathfrak{m}_{X, x} / \mathfrak{m}_{X, x}^{2}\right)^{*}$ to be the Zariski tangent space, i.e. the tangent space of a minimal smooth ambient vector space containing the germ $(X, x)$; all tangent vectors may not be reached by analytic disks and in those cases we put $\mathbf{k}_{X}(\xi)=+\infty$. When $X$ is a smooth manifold, it follows from the work of H.L. Royden ([Roy71], [Roy74]) that $\mathbf{k}_{X}$ is upper-continuous on $T_{X}$ and that $d_{X}^{K}$ is the integrated pseudodistance associated with the pseudometric, i.e.

$$
d_{X}^{K}(p, q)=\inf _{\gamma} \int_{\gamma} \mathbf{k}_{X}\left(\gamma^{\prime}(t)\right) d t
$$

where the infimum is taken over all piecewise smooth curves joining $p$ to $q$; in the case of complex spaces, a similar formula holds, involving jets of analytic curves of arbitrary order, cf. S. Venturini [Ven96]. When $X$ is a non-singular projective variety, it has been shown in [DeLS94] that the Kobayashi pseudodistance and the Kobayashi-Royden infinitesimal pseudometric can be computed by looking only at analytic disks that are contained in algebraic curves.

1.3. Definition. A complex space $X$ is said to be hyperbolic (in the sense of Kobayashi) if $d_{X}^{K}$ is actually a distance, namely if $d_{X}^{K}(p, q)>0$ for all pairs of distinct points $(p, q)$ in $X$.

When $X$ is hyperbolic, it is interesting to investigate when the Kobayashi metric is complete: one then says that $X$ is a complete hyperbolic space. However, we will be mostly concerned with compact spaces here, so completeness is irrelevant in that case.

Another important property is the monotonicity of the Kobayashi pseudometric with respect to holomorphic mappings. In fact, if $\Phi: X \rightarrow Y$ is a holomorphic map, it is easy to see from the definition that

$$
d_{Y}^{K}(\Phi(p), \Phi(q)) \leqslant d_{X}^{K}(p, q), \quad \text { for all } p, q \in X .
$$

The proof merely consists of taking the composition $\Phi \circ f_{i}$ for all chains of analytic disks connecting $p$ and $q$ in $X$. Clearly the Kobayashi pseudodistance $d_{\mathbb{C}}^{K}$ on $X=\mathbb{C}$ is identically zero, as one can see by looking at arbitrarily large analytic disks $\Delta \rightarrow \mathbb{C}, t \mapsto \lambda t$. Therefore, if there is an entire curve $\Phi: \mathbb{C} \rightarrow X$, namely a non-constant holomorphic map defined on the whole complex plane $\mathbb{C}$, then by monotonicity $d_{X}^{K}$ is identically zero on the image $\Phi(\mathbb{C})$ of the curve, and therefore $X$ cannot be hyperbolic. When $X$ is hyperbolic, it follows that $X$ cannot contain rational curves $C \simeq \mathbb{P}^{1}$, or elliptic curves $\mathbb{C} / \Lambda$, or more generally any non-trivial image $\Phi: W=\mathbb{C}^{p} / \Lambda \rightarrow X$ of a $p$-dimensional complex torus (quotient of $\mathbb{C}^{p}$ by a lattice). The only case where hyperbolicity is easy to assess is the case of curves $\left(\operatorname{dim}_{\mathbb{C}} X=1\right)$.

1.5. Case of complex curves. Up to bihomorphism, any smooth complex curve $X$ belongs to one (and only one) of the following three types:

(a) (rational curve) $X \simeq \mathbb{P}^{1}$;

(b) $($ parabolic type $) \hat{X} \simeq \mathbb{C}, X \simeq \mathbb{C}, \mathbb{C}^{*}$ or $X \simeq \mathbb{C} / \Lambda$ (elliptic curve $)$;

(c) (hyperbolic type) $\widehat{X} \simeq \Delta$. All compact curves $X$ of genus $g \geqslant 2$ enter in this category, as well as $X=\mathbb{P}^{1} \backslash\{a, b, c\} \simeq \mathbb{C} \backslash\{0,1\}$, or $X=\mathbb{C} / \Lambda \backslash\{a\}$ (elliptic curve minus one point).

In fact, as the disk is simply connected, every holomorphic map $f: \Delta \rightarrow X$ lifts to the universal cover $\widehat{f}: \Delta \rightarrow \widehat{X}$, so that $f=\rho \circ \widehat{f}$ where $\rho: \widehat{X} \rightarrow X$ is the projection map, and the conclusions $(\mathrm{a}, \mathrm{b}, \mathrm{c})$ follow easily from the Poincaré-Koebe uniformization theorem: every simply connected Riemann surface is biholomorphic to $\mathbb{C}$, the unit disk $\Delta$ or the complex projective line $\mathbb{P}^{1}$.

In some rare cases, the one-dimensional case can be used to study the case of higher dimensions. For instance, it is easy to see by looking at projections that the Kobayashi pseudodistance on a 
product $X \times Y$ of complex spaces is given by

$$
\begin{aligned}
& d_{X \times Y}^{K}\left((x, y),\left(x^{\prime}, y^{\prime}\right)\right)=\max \left(d_{X}^{K}\left(x, x^{\prime}\right), d_{Y}^{K}\left(y, y^{\prime}\right)\right), \\
& \mathbf{k}_{X \times Y}\left(\xi, \xi^{\prime}\right)=\max \left(\mathbf{k}_{X}(\xi), \mathbf{k}_{Y}\left(\xi^{\prime}\right)\right),
\end{aligned}
$$

and from there it follows that a product of hyperbolic spaces is hyperbolic. As a consequence $(\mathbb{C} \backslash\{0,1\})^{2}$, which is also a complement of five lines in $\mathbb{P}^{2}$, is hyperbolic.

\section{B. BRODY CRITERION FOR HYPERBOLICITY}

Throughout this subsection, we assume that $X$ is a complex manifold. In this context, we have the following well-known result of Brody [Bro78]. Its main interest is to relate hyperbolicity to the non-existence of entire curves.

1.7. Brody reparametrization lemma. Let $\omega$ be a Hermitian metric on $X$ and let $f: \Delta \rightarrow X$ be a holomorphic map. For every $\varepsilon>0$, there exists a radius $R \geqslant(1-\varepsilon)\left\|f^{\prime}(0)\right\|_{\omega}$ and a homographic transformation $\psi$ of the disk $D(0, R)$ onto $(1-\varepsilon) \Delta$ such that

$$
\left\|(f \circ \psi)^{\prime}(0)\right\|_{\omega}=1, \quad\left\|(f \circ \psi)^{\prime}(t)\right\|_{\omega} \leqslant \frac{1}{1-|t|^{2} / R^{2}} \quad \text { for every } t \in D(0, R) .
$$

Proof. Select $t_{0} \in \Delta$ such that $\left(1-|t|^{2}\right)\left\|f^{\prime}((1-\varepsilon) t)\right\|_{\omega}$ reaches its maximum for $t=t_{0}$. The reason for this choice is that $\left(1-|t|^{2}\right)\left\|f^{\prime}((1-\varepsilon) t)\right\|_{\omega}$ is the norm of the differential $f^{\prime}((1-\varepsilon) t): T_{\Delta} \rightarrow T_{X}$ with respect to the Poincaré metric $|d t|^{2} /\left(1-|t|^{2}\right)^{2}$ on $T_{\Delta}$, which is conformally invariant under Aut $(\Delta)$. One then adjusts $R$ and $\psi$ so that $\psi(0)=(1-\varepsilon) t_{0}$ and $\left|\psi^{\prime}(0)\right|\left\|f^{\prime}(\psi(0))\right\|_{\omega}=1$. As $\left|\psi^{\prime}(0)\right|=\frac{1-\varepsilon}{R}\left(1-\left|t_{0}\right|^{2}\right)$, the only possible choice for $R$ is

$$
R=(1-\varepsilon)\left(1-\left|t_{0}\right|^{2}\right)\left\|f^{\prime}(\psi(0))\right\|_{\omega} \geqslant(1-\varepsilon)\left\|f^{\prime}(0)\right\|_{\omega} .
$$

The inequality for $(f \circ \psi)^{\prime}$ follows from the fact that the Poincaré norm is maximum at the origin, where it is equal to 1 by the choice of $R$.

Using the Ascoli-Arzelà theorem we obtain immediately:

1.8. Corollary (Brody). Let $(X, \omega)$ be a compact complex Hermitian manifold. Given a sequence of holomorphic mappings $f_{\nu}: \Delta \rightarrow X$ such that $\lim \left\|f_{\nu}^{\prime}(0)\right\|_{\omega}=+\infty$, one can find a sequence of homographic transformations $\psi_{\nu}: D\left(0, R_{\nu}\right) \rightarrow(1-1 / \nu) \Delta$ with $\lim R_{\nu}=+\infty$, such that, after passing possibly to a subsequence, $\left(f_{\nu} \circ \psi_{\nu}\right)$ converges uniformly on every compact subset of $\mathbb{C}$ towards a non-constant holomorphic map $g: \mathbb{C} \rightarrow X$ with $\left\|g^{\prime}(0)\right\|_{\omega}=1$ and $\sup _{t \in \mathbb{C}}\left\|g^{\prime}(t)\right\|_{\omega} \leqslant 1$.

An entire curve $g: \mathbb{C} \rightarrow X$ such that $\sup _{\mathbb{C}}\left\|g^{\prime}\right\|_{\omega}=M<+\infty$ is called a Brody curve; this concept does not depend on the choice of $\omega$ when $X$ is compact, and one can always assume $M=1$ by rescaling the parameter $t$.

1.9. Brody criterion. Let $X$ be a compact complex manifold. The following properties are equivalent:

(a) $X$ is hyperbolic;

(b) $X$ does not possess any entire curve $f: \mathbb{C} \rightarrow X$;

(c) $X$ does not possess any Brody curve $g: \mathbb{C} \rightarrow X$;

(d) The Kobayashi infinitesimal metric $\mathbf{k}_{X}$ is uniformly bounded below, namely

$$
\mathbf{k}_{X}(\xi) \geqslant c\|\xi\|_{\omega}, \quad c>0,
$$

for any Hermitian metric $\omega$ on $X$.

Proof. (a) $\Rightarrow$ (b). If $X$ possesses an entire curve $f: \mathbb{C} \rightarrow X$, then by looking at arbitrary large disks $D(0, R) \subset \mathbb{C}$, it is easy to see that the Kobayashi distance of any two points in $f(\mathbb{C})$ is zero, so $X$ is not hyperbolic.

(b) $\Rightarrow(c)$. This is trivial. 
(c) $\Rightarrow$ (d). If (d) does not hold, there exists a sequence of tangent vectors $\xi_{\nu} \in T_{X, x_{\nu}}$ with $\left\|\xi_{\nu}\right\|_{\omega}=1$ and $\mathbf{k}_{X}\left(\xi_{\nu}\right) \rightarrow 0$. By definition, this means that there exists an analytic curve $f_{\nu}: \Delta \rightarrow X$ with $f(0)=x_{\nu}$ and $\left\|f_{\nu}^{\prime}(0)\right\|_{\omega} \geqslant\left(1-\frac{1}{\nu}\right) / \mathbf{k}_{X}\left(\xi_{\nu}\right) \rightarrow+\infty$. One can then produce a Brody curve $g=\mathbb{C} \rightarrow X$ by Corollary 1.8, contradicting (c).

$(d) \Rightarrow(\mathrm{a})$. In fact $(\mathrm{d})$ implies after integrating that $d_{X}^{K}(p, q) \geqslant c d_{\omega}(p, q)$ where $d_{\omega}$ is the geodesic distance associated with $\omega$, so $d_{X}^{K}$ must be non degenerate.

Notice also that if $f: \mathbb{C} \rightarrow X$ is an entire curve such that $\left\|f^{\prime}\right\|_{\omega}$ is unbounded, one can apply the Corollary 1.8 to $f_{\nu}(t):=f\left(t+a_{\nu}\right)$ where the sequence $\left(a_{\nu}\right)$ is chosen such that $\left\|f_{\nu}^{\prime}(0)\right\|_{\omega}=$ $\left\|f\left(a_{\nu}\right)\right\|_{\omega} \rightarrow+\infty$. Brody's result then produces reparametrizations $\psi_{\nu}: D\left(0, R_{\nu}\right) \rightarrow D\left(a_{\nu}, 1-1 / \nu\right)$ and a Brody curve $g=\lim f \circ \psi_{\nu}: \mathbb{C} \rightarrow X$ such that sup $\left\|g^{\prime}\right\|_{\omega}=1$ and $g(\mathbb{C}) \subset \overline{f(\mathbb{C})}$. It may happen that the image $g(\mathbb{C})$ of such a limiting curve is disjoint from $f(\mathbb{C})$. In fact Winkelmann [Win07] has given a striking example, actually a projective 3-fold $X$ obtained by blowing-up a 3-dimensional abelian variety $Y$, such that every Brody curve $g: \mathbb{C} \rightarrow X$ lies in the exceptional divisor $E \subset X$; however, entire curves $f: \mathbb{C} \rightarrow X$ can be dense, as one can see by taking $f$ to be the lifting of a generic complex line embedded in the abelian variety $Y$. For further precise information on the localization of Brody curves, we refer the reader to the remarkable results of [Duv08].

The absence of entire holomorphic curves in a given complex manifold is often referred to as Brody hyperbolicity. Thus, in the compact case, Brody hyperbolicity and Kobayashi hyperbolicity coincide (but Brody hyperbolicity is in general a strictly weaker property when $X$ is non-compact).

\section{C. Geometric applichtions}

We give here two immediate consequences of the Brody criterion: the openness property of hyperbolicity and a hyperbolicity criterion for subvarieties of complex tori. By definition, a holomorphic family of compact complex manifolds is a holomorphic proper submersion $\mathcal{X} \rightarrow S$ between two complex manifolds.

1.10. Proposition. Let $\pi: \mathcal{X} \rightarrow S$ be a holomorphic family of compact complex manifolds. Then the set of $s \in S$ such that the fiber $X_{s}=\pi^{-1}(s)$ is hyperbolic is open in the Euclidean topology.

Proof. Let $\omega$ be an arbitrary Hermitian metric on $\mathcal{X},\left(X_{s_{\nu}}\right)_{s_{\nu} \in S}$ a sequence of non-hyperbolic fibers, and $s=\lim s_{\nu}$. By the Brody criterion, one obtains a sequence of entire maps $f_{\nu}: \mathbb{C} \rightarrow X_{s_{\nu}}$ such that $\left\|f_{\nu}^{\prime}(0)\right\|_{\omega}=1$ and $\left\|f_{\nu}^{\prime}\right\|_{\omega} \leqslant 1$. Ascoli's theorem shows that there is a subsequence of $f_{\nu}$ converging uniformly to a limit $f: \mathbb{C} \rightarrow X_{s}$, with $\left\|f^{\prime}(0)\right\|_{\omega}=1$. Hence $X_{s}$ is not hyperbolic and the collection of non-hyperbolic fibers is closed in $S$.

Consider now an $n$-dimensional complex torus $W$, i.e. an additive quotient $W=\mathbb{C}^{n} / \Lambda$, where $\Lambda \subset \mathbb{C}^{n}$ is a (cocompact) lattice. By taking a composition of entire curves $\mathbb{C} \rightarrow \mathbb{C}^{n}$ with the projection $\mathbb{C}^{n} \rightarrow W$ we obtain an infinite dimensional space of entire curves in $W$.

1.11. Theorem. Let $X \subset W$ be a compact complex submanifold of a complex torus. Then $X$ is hyperbolic if and only if it does not contain any translate of a subtorus.

Proof. If $X$ contains some translate of a subtorus, then it contains lots of entire curves and so $X$ is not hyperbolic.

Conversely, suppose that $X$ is not hyperbolic. Then by the Brody criterion there exists an entire curve $f: \mathbb{C} \rightarrow X$ such that $\left\|f^{\prime}\right\|_{\omega} \leqslant\left\|f^{\prime}(0)\right\|_{\omega}=1$, where $\omega$ is the flat metric on $W$ inherited from $\mathbb{C}^{n}$. This means that any lifting $\widetilde{f}=\left(\widetilde{f}, \ldots, \widetilde{f}_{\nu}\right): \mathbb{C} \rightarrow \mathbb{C}^{n}$ is such that

$$
\sum_{j=1}^{n}\left|f_{j}^{\prime}\right|^{2} \leqslant 1 .
$$

Then, by Liouville's theorem, $\tilde{f}^{\prime}$ is constant and therefore $\tilde{f}$ is affine linear. But then the closure of the image of $f$ is a translate $a+H$ of a connected (possibly real) subgroup $H$ of $W$. We conclude 
that $X$ contains the analytic Zariski closure of $a+H$, namely $a+H^{\mathbb{C}}$ where $H^{\mathbb{C}} \subset W$ is the smallest closed complex subgroup of $W$ containing $H$.

\section{DiRECTED MANIFOLDS}

\section{A. BASIC DEFINITIONS CONCERNING DIRECTED MANIFOLDS}

Let us consider a pair $(X, V)$ consisting of an $n$-dimensional complex manifold $X$ equipped with a linear subspace $V \subset T_{X}$ : if we assume $X$ to be connected, this is by definition an irreducible closed analytic subspace of the total space of $T_{X}$ such that each fiber $V_{x}=V \cap T_{X, x}$ is a vector subspace of $T_{X, x}$. If $\mathcal{W} \subset \Omega_{X}^{1}$ is the sheaf of 1 -forms vanishing on $V$, then $\mathcal{W}$ is coherent (this follows from the direct image theorem by looking at the proper morphism $\left.P(V) \subset P\left(T_{X}\right) \rightarrow X\right)$, and $V$ is locally defined by

$$
V_{x}=\left\{\xi \in T_{X, x} ; \alpha_{j}(x) \cdot \xi=0,1 \leqslant j \leqslant N\right\}, \quad \alpha_{j} \in H^{0}\left(U, \Omega_{X}^{1}\right), \quad x \in U,
$$

where $\left(\alpha_{1}, \ldots, \alpha_{N}\right)$, is a local family of generators of $\mathcal{W}$ on a small open set $U$. We can also associate to $V$ a coherent sheaf $\mathcal{V}:=\mathcal{W}^{\perp}=\operatorname{Hom}\left(\Omega_{X}^{1} / \mathcal{W}, \mathcal{O}_{X}\right) \subset \mathcal{O}\left(T_{X}\right)$, which is a saturated subsheaf of $\mathcal{O}\left(T_{X}\right)$, i.e. such that $\mathcal{O}\left(T_{X}\right) / \mathcal{V}$ has no torsion; then $\mathcal{V}$ is also reflexive, i.e. $\mathcal{V}^{* *}=\mathcal{V}$. We will refer to such a pair as being a (complex) directed manifold, and we will in general think of $V$ as a linear space (rather than considering the associated saturated subsheaf $\mathcal{V} \subset \mathcal{O}\left(T_{X}\right)$ ). A morphism $\Phi:(X, V) \rightarrow(Y, W)$ in the category of complex directed manifolds is a holomorphic map such that $\Phi_{*}(V) \subset W$.

Here, the rank $x \mapsto \operatorname{dim}_{\mathbb{C}} V_{x}$ is Zariski lower semi-continuous, and it may a priori jump. The $\operatorname{rank} r:=\operatorname{rank}(V) \in\{0,1, \ldots, n\}$ of $V$ is by definition the dimension of $V_{x}$ at a generic point. The dimension may be larger at non-generic points; this happens e.g. on $X=\mathbb{C}^{n}$ for the rank 1 linear space $V$ generated by the Euler vector field: $V_{z}=\mathbb{C} \sum_{1 \leqslant j \leqslant n} z_{j} \frac{\partial}{\partial z_{j}}$ for $z \neq 0$, and $V_{0}=\mathbb{C}^{n}$. Our philosophy is that directed manifolds are also useful to study the "absolute case", i.e. the case $V=T_{X}$, because there are certain functorial constructions which are quite natural in the category of directed manifolds (see e.g. $\S 5,6,7$ ). We think of directed manifolds as a kind of "relative situation", covering e.g. the case when $V$ is the relative tangent space to a holomorphic map $X \rightarrow S$. It is important to notice that the local sections of $\mathcal{V}$ need not generate the fibers of $V$ at singular points, as one sees already in the case of the Euler vector field when $n \geqslant 2$. We also want to stress that no assumption need be made on the Lie bracket tensor $[\bullet, \bullet]: \mathcal{V} \times \mathcal{V} \rightarrow \mathcal{O}\left(T_{X}\right) / \mathcal{V}$, i.e. we do not assume any kind of integrability for $\mathcal{V}$ or $\mathcal{W}$.

The singular set $\operatorname{Sing}(V)$ is by definition the set of points where $\mathcal{V}$ is not locally free, it can also be defined as the indeterminacy set of the (meromorphic) classifying map $\alpha: X \rightarrow G_{r}\left(T_{X}\right)$, $z \mapsto V_{z}$ to the Grassmannian of $r$ dimensional subspaces of $T_{X}$. We thus have $V_{\uparrow X \backslash \operatorname{Sing}(V)}=\alpha^{*} S$ where $S \rightarrow G_{r}\left(T_{X}\right)$ is the tautological subbundle of $G_{r}\left(T_{X}\right)$. The singular set $\operatorname{Sing}(V)$ is an analytic subset of $X$ of codim $\geqslant 2$, and hence $V$ is always a holomorphic subbundle outside of codimension 2 . Thanks to this remark, one can most often treat linear spaces as vector bundles (possibly modulo passing to the Zariski closure along $\operatorname{Sing}(V)$ ).

\section{B. HyPERBolicity PROPERTIES OF DIRECTED MANIFOLDS}

Most of what we have done in $\S 1$ can be extended to the category of directed manifolds.

2.1. Definition. Let $(X, V)$ be a complex directed manifold.

(i) The Kobayashi-Royden infinitesimal metric of $(X, V)$ is the Finsler metric on $V$ defined for any $x \in X$ and $\xi \in V_{x}$ by

$$
\mathbf{k}_{(X, V)}(\xi)=\inf \left\{\lambda>0 ; \exists f: \Delta \rightarrow X, f(0)=x, \lambda f^{\prime}(0)=\xi, f^{\prime}(\Delta) \subset V\right\} .
$$

Here $\Delta \subset \mathbb{C}$ is the unit disk and the map $f$ is an arbitrary holomorphic map which is tangent to $V$, i.e., such that $f^{\prime}(t) \in V_{f(t)}$ for all $t \in \Delta$. We say that $(X, V)$ is infinitesimally hyperbolic if $\mathbf{k}_{(X, V)}$ is positive definite on every fiber $V_{x}$ and satisfies a uniform lower bound $\mathbf{k}_{(X, V)}(\xi) \geqslant$ 
$\varepsilon\|\xi\|_{\omega}$ in terms of any smooth Hermitian metric $\omega$ on $X$, when $x$ describes a compact subset of $X$.

(ii) More generally, the Kobayashi-Eisenman infinitesimal pseudometric of $(X, V)$ is the pseudometric defined on all decomposable p-vectors $\xi=\xi_{1} \wedge \cdots \wedge \xi_{p} \in \Lambda^{p} V_{x}, 1 \leqslant p \leqslant r=\operatorname{rank}(V)$, by

$$
\mathbf{e}_{(X, V)}^{p}(\xi)=\inf \left\{\lambda>0 ; \exists f: \mathbb{B}_{p} \rightarrow X, f(0)=x, \lambda f_{*}\left(\tau_{0}\right)=\xi, f_{*}\left(T_{\mathbb{B}_{p}}\right) \subset V\right\},
$$

where $\mathbb{B}_{p}$ is the unit ball in $\mathbb{C}^{p}$ and $\tau_{0}=\partial / \partial t_{1} \wedge \cdots \wedge \partial / \partial t_{p}$ is the unit p-vector of $\mathbb{C}^{p}$ at the origin. We say that $(X, V)$ is infinitesimally p-measure hyperbolic if $\mathbf{e}_{(X, V)}^{p}$ is positive definite on every fiber $\Lambda^{p} V_{x}$ and satisfies a locally uniform lower bound in terms of any smooth metric.

If $\Phi:(X, V) \rightarrow(Y, W)$ is a morphism of directed manifolds, it is immediate to check that we have the monotonicity property

$$
\begin{aligned}
& \mathbf{k}_{(Y, W)}\left(\Phi_{*} \xi\right) \leqslant \mathbf{k}_{(X, V)}(\xi), \quad \forall \xi \in V, \\
& \mathbf{e}_{(Y, W)}^{p}\left(\Phi_{*} \xi\right) \leqslant \mathbf{e}_{(X, V)}^{p}(\xi), \quad \forall \xi=\xi_{1} \wedge \cdots \wedge \xi_{p} \in \Lambda^{p} V .
\end{aligned}
$$

The following proposition shows that virtually all reasonable definitions of the hyperbolicity property are equivalent if $X$ is compact (in particular, the additional assumption that there is locally uniform lower bound for $\mathbf{k}_{(X, V)}$ is not needed). We merely say in that case that $(X, V)$ is hyperbolic.

2.3. Proposition. For an arbitrary directed manifold $(X, V)$, the Kobayashi-Royden infinitesimal metric $\mathbf{k}_{(X, V)}$ is upper semi-continuous on the total space of $V$. If $X$ is compact, $(X, V)$ is infinitesimally hyperbolic if and only if there are no entire curves $g: \mathbb{C} \rightarrow X$ tangent to $V$. In that case, $\mathbf{k}_{(X, V)}$ is a continuous (and positive definite) Finsler metric on $V$.

Proof. The proof is almost identical to the standard proof for $\mathbf{k}_{X}$, for which we refer to Royden [Roy71, Roy74]. One of the main ingredients is that one can find a Stein neighborhood of the graph of any analytic disk (thanks to a result of [Siu76], cf. also [Dem90a] for more general results). This allows to obtain "free" small deformations of any given analytic disk, as there are many holomorphic vector fields on a Stein manifold.

Another easy observation is that the concept of $p$-measure hyperbolicity gets weaker and weaker as $p$ increases (we leave it as an exercise to the reader, this is mostly just linear algebra).

2.4. Proposition. If $(X, V)$ is p-measure hyperbolic, then it is $(p+1)$-measure hyperbolic for all $p \in\{1, \ldots, \operatorname{rank}(V)-1\}$.

Again, an argument extremely similar to the proof of 1.10 shows that relative hyperbolicity is an open property.

2.5. Proposition. Let $(\mathcal{X}, \mathcal{V}) \rightarrow S$ be a holomorphic family of compact directed manifolds (by this, we mean a proper holomorphic map $\mathcal{X} \rightarrow S$ together with an analytic linear subspace $\mathcal{V} \subset T_{\mathcal{X} / S} \subset$ $T_{\mathcal{X}}$ of the relative tangent bundle, defining a deformation $\left(X_{s}, V_{s}\right)_{s \in S}$ of the fibers). Then the set of $s \in S$ such that the fiber $\left(X_{s}, V_{s}\right)$ is hyperbolic is open in $S$ with respect to the Euclidean topology.

Let us mention here an impressive result proved by Marco Brunella [Bru03, Bru05, Bru06] concerning the behavior of the Kobayashi metric on foliated varieties.

2.6. Theorem (Brunella). Let $X$ be a compact Kähler manifold equipped with a (possibly singular) rank 1 holomorphic foliation which is not a foliation by rational curves. Then the canonical bundle $K_{\mathcal{F}}=\mathcal{F}^{*}$ of the foliation is pseudoeffective (i.e. the curvature of $K_{\mathcal{F}}$ is $\geqslant 0$ in the sense of currents).

The proof is obtained by putting on $K_{\mathcal{F}}$ precisely the metric induced by the Kobayashi metric on the leaves whenever they are generically hyperbolic (i.e. covered by the unit disk). The case of parabolic leaves (covered by $\mathbb{C}$ ) has to be treated separately. 
Let $(X, V)$ be a directed projective manifold where $V$ is possibly singular, and let $r=\operatorname{rank} V$. If $\mu: \widehat{X} \rightarrow X$ is a proper modification (a composition of blow-ups with smooth centers, say), we get a directed manifold $(\widehat{X}, \widehat{V})$ by taking $\widehat{V}$ to be the closure of $\mu_{*}^{-1}\left(V^{\prime}\right)$, where $V^{\prime}=V_{\uparrow X^{\prime}}$ is the restriction of $V$ over a Zariski open set $X^{\prime} \subset X \backslash \operatorname{Sing}(V)$ such that $\mu: \mu^{-1}\left(X^{\prime}\right) \rightarrow X^{\prime}$ is a biholomorphism. We say that $(\widehat{X}, \widehat{V})$ is a modification of $(X, V)$ and write $\widehat{V}=\mu^{*} V$.

We will be interested in taking modifications realized by iterated blow-ups of certain non-singular subvarieties of the singular set $\operatorname{Sing}(V)$, so as to eventually "improve" the singularities of $V$; outside of $\operatorname{Sing}(V)$ the effect of blowing-up will be irrelevant. The canonical sheaf $K_{V}$, resp. the pluricanonical sheaf sequence $K_{V}^{[m]}$, will be defined here in several steps, using the concept of bounded pluricanonical forms that was already introduced in [Dem11].

2.7. Definition. For a directed pair $(X, V)$ with $X$ non-singular, we define ${ }^{b} K_{V}$, resp. ${ }^{b} K_{V}^{[m]}$, for any integer $m \geqslant 0$, to be the rank 1 analytic sheaves such that

$$
\begin{aligned}
{ }^{b} K_{V}(U) & =\text { sheaf of locally bounded sections of } \mathcal{O}_{X}\left(\Lambda^{r} V^{* *}\right)\left(U \cap X^{\prime}\right), \\
{ }^{b} K_{V}^{[m]}(U) & =\text { sheaf of locally bounded sections of } \mathcal{O}_{X}\left(\left(\Lambda^{r} V^{\prime *}\right)^{\otimes m}\right)\left(U \cap X^{\prime}\right),
\end{aligned}
$$

where $r=\operatorname{rank}(V), X^{\prime}=X \backslash \operatorname{Sing}(V), V^{\prime}=V_{\uparrow X^{\prime}}$, and "locally bounded" means bounded with respect to a smooth Hermitian metric $h$ on $T_{X}$, on every set $W \cap X^{\prime}$ such that $W$ is relatively compact in $U$.

In the trivial case $r=0$, we simply set ${ }^{b} K_{V}^{[m]}=\mathcal{O}_{X}$ for all $m$; clearly $\operatorname{ECL}(X, V)=\emptyset$ in that case, so there is not much to say. The above definition of ${ }^{b} K_{V}^{[m]}$ may look like an analytic one, but it can easily be turned into an equivalent algebraic definition:

2.8. Proposition. Consider the natural morphism $\mathcal{O}\left(\Lambda^{r} T_{X}^{*}\right) \rightarrow \mathcal{O}\left(\Lambda^{r} V^{*}\right)$ where $r=\operatorname{rank} V$ and $\mathcal{O}\left(\Lambda^{r} V^{*}\right)$ is defined as the quotient of $\mathcal{O}\left(\Lambda^{r} T_{X}^{*}\right)$ by r-forms that have zero restrictions to $\mathcal{O}\left(\Lambda^{r} V^{*}\right)$ on $X \backslash \operatorname{Sing}(V)$. The bidual $\mathcal{L}_{V}=\mathcal{O}_{X}\left(\Lambda^{r} V^{*}\right)^{* *}$ is an invertible sheaf, and our natural morphism can be written

$$
\mathcal{O}\left(\Lambda^{r} T_{X}^{*}\right) \rightarrow \mathcal{O}\left(\Lambda^{r} V^{*}\right)=\mathcal{L}_{V} \otimes \mathcal{J}_{V} \subset \mathcal{L}_{V}
$$

where $\mathcal{J}_{V}$ is a certain ideal sheaf of $\mathcal{O}_{X}$ whose zero set is contained in $\operatorname{Sing}(V)$, and the arrow on the left is surjective by definition. Then

$$
{ }^{b} K_{V}^{[m]}=\mathcal{L}_{V}^{\otimes m} \otimes \overline{\mathcal{J}_{V}^{m}}
$$

where $\overline{\mathcal{J}_{V}^{m}}$ is the integral closure of $\mathcal{J}_{V}^{m}$ in $\mathcal{O}_{X}$. In particular, ${ }^{b} K_{V}^{[m]}$ is always a coherent sheaf.

Proof. Let $\left(u_{k}\right)$ be a set of generators of $\mathcal{O}\left(\Lambda^{r} V^{*}\right)$ obtained (say) as the images of a basis $\left(d z_{I}\right)_{|I|=r}$ of $\Lambda^{r} T_{X}^{*}$ in some local coordinates near a point $x \in X$. Write $u_{k}=g_{k} \ell$ where $\ell$ is a local generator of $\mathcal{L}_{V}$ at $x$. Then $\mathcal{J}_{V}=\left(g_{k}\right)$ by definition. The boundedness condition expressed in Def. 2.7 means that we take sections of the form $f \ell^{\otimes m}$ where $f$ is a holomorphic function on $U \cap X^{\prime}$ (and $U$ a neighborhood of $x$ ), such that

$$
|f| \leqslant C\left(\sum\left|g_{k}\right|\right)^{m}
$$

for some constant $C>0$. But then $f$ extends holomorphically to $U$ into a function that lies in the integral closure $\overline{\mathcal{J}}_{V}^{m}$ (it is well known that the latter is characterized analytically by condition $\left(2.8_{3}\right)$ ). This proves Prop. 2.8 .

2.9. Lemma. Let $(X, V)$ be a directed variety.

(a) For any modification $\mu:(\widehat{X}, \widehat{V}) \rightarrow(X, V)$, there are always well defined injective natural morphisms of rank 1 sheaves

$$
{ }^{b} K_{V}^{[m]} \hookrightarrow \mu_{*}\left({ }^{b} K_{\widehat{V}}^{[m]}\right) \hookrightarrow \mathcal{L}_{V}^{\otimes m}
$$


(b) The direct image $\mu_{*}\left({ }^{b} K_{\widehat{V}}^{[m]}\right)$ may only increase when we replace $\mu$ by a "higher" modification $\widetilde{\mu}=\mu^{\prime} \circ \mu: \widetilde{X} \rightarrow \widehat{X} \rightarrow X$ and $\widehat{V}=\mu^{*} V$ by $\widetilde{V}=\widetilde{\mu}^{*} V$, i.e. there are injections

$$
\mu_{*}\left({ }^{b} K_{\widehat{V}}^{[m]}\right) \hookrightarrow \widetilde{\mu}_{*}\left({ }^{b} K_{\widetilde{V}}^{[m]}\right) \hookrightarrow \mathcal{L}_{V}^{\otimes m} .
$$

We refer to this property as the monotonicity principle.

Proof. (a) The existence of the first arrow is seen as follows: the differential $\mu_{*}=d \mu: \widehat{V} \rightarrow \mu^{*} V$ is smooth, so it is bounded with respect to ambient Hermitian metrics on $X$ and $\widehat{X}$. Going to the duals reverses the arrows while preserving boundedness with respect to the metrics. We thus get an arrow

$$
\mu^{*}\left({ }^{b} V^{\star}\right) \hookrightarrow{ }^{b} \widehat{V}^{\star} .
$$

By taking the top exterior power, followed by the $m$-th tensor product and the integral closure of the ideals involved, we get an injective arrow $\mu^{*}\left({ }^{b} K_{V}^{[m]}\right) \hookrightarrow{ }^{b} K_{\widehat{V}}^{[m]}$. Finally we apply the direct image functor $\mu_{*}$ and the canonical morphism $\mathcal{F} \rightarrow \mu_{*} \mu^{*} \mathcal{F}$ to get the first inclusion morphism. The second arrow comes from the fact that $\mu^{*}\left({ }^{b} K_{V}^{[m]}\right)$ coincides with $\mathcal{L}_{V}^{\otimes m}$ (and with $\left.\operatorname{det}\left(V^{*}\right)^{\otimes m}\right)$ on the complement of the codimension 2 set $S=\operatorname{Sing}(V) \cup \mu(\operatorname{Exc}(\mu))$, and the fact that for every open set $U \subset X$, sections of $\mathcal{L}_{V}$ defined on $U \backslash S$ automatically extend to $U$ by Riemann's extension theorem (or Hartog's extension theorem ...), even without any boundedness assumption.

(b) Given $\mu^{\prime}: \widetilde{X} \rightarrow \widehat{X}$, we argue as in (a) that there is a bounded morphism $d \mu^{\prime}: \widetilde{V} \rightarrow \widehat{V}$.

By the monotonicity principle and the strong Noetherian property of coherent ideals, we infer that there exists a maximal direct image when $\mu: \widehat{X} \rightarrow X$ runs over all non-singular modifications of $X$. The following definition is thus legitimate.

2.10. Definition. We define the pluricanonical sheaves $K_{V}^{m}$ of $(X, V)$ to be the inductive limits

$$
K_{V}^{[m]}:=\underset{\mu}{\lim } \mu_{*}\left({ }^{b} K_{\widehat{V}}^{[m]}\right)=\max _{\mu} \mu_{*}\left({ }^{b} K_{\widehat{V}}^{[m]}\right)
$$

taken over the family of all modifications $\mu:(\widehat{X}, \widehat{V}) \rightarrow(X, V)$, with the trivial (filtering) partial order. The canonical sheaf $K_{V}$ itself is defined to be the same as $K_{V}^{[1]}$. By construction, we have for every $m \geqslant 0$ inclusions

$$
{ }^{b} K_{V}^{[m]} \hookrightarrow K_{V}^{[m]} \hookrightarrow \mathcal{L}_{V}^{\otimes m}
$$

and $K_{V}^{[m]}=\mathcal{J}_{V}^{[m]} \cdot \mathcal{L}_{V}^{\otimes m}$ for a certain sequence of integrally closed ideals $\mathcal{J}_{V}^{[m]} \subset \mathcal{O}_{X}$.

It is clear from this construction that $K_{V}^{[m]}$ is birationally invariant, i.e. that $K_{V}^{[m]}=\mu_{*}\left(K_{V^{\prime}}^{[m]}\right)$ for every modification $\mu:\left(X^{\prime}, V^{\prime}\right) \rightarrow(X, V)$. Moreover the sequence is submultiplicative, i.e. there are injections

$$
K_{V}^{\left[m_{1}\right]} \otimes K_{V}^{\left[m_{2}\right]} \hookrightarrow K_{V}^{\left[m_{1}+m_{2}\right]}
$$

for all non-negative integers $m_{1}, m_{2}$; the corresponding sequence of ideals $\mathcal{J}_{V}^{[m]}$ is thus also submultiplicative. By blowing up $\mathcal{J}_{V}^{[m]}$ and taking a desingularization $\widehat{X}$ of the blow-up, one can always find a log-resolution of $\mathcal{J}_{V}^{[m]}$, i.e. a modification $\mu_{m}: \widehat{X}_{m} \rightarrow X$ such that $\mu_{m}^{*} \mathcal{J}_{V}^{[m]} \subset \mathcal{O}_{\widehat{X}_{m}}$ is an invertible ideal sheaf; it follows that

$$
\mu_{m}^{*} K_{V}^{[m]}=\mu_{m}^{*} \mathcal{J}_{V}^{[m]} \cdot\left(\mu_{m}^{*} \mathcal{L}_{V}\right)^{\otimes m}
$$

is an invertible sheaf on $\widehat{X}_{m}$. We do not know whether $\mu_{m}$ can be taken independent of $m$, nor whether the inductive limit introduced in Definition 2.10 is reached for a $\mu$ that is independent of $m$. If such a "uniform" $\mu$ exists, it could be thought of as some sort of replacement for the resolution of singularities of directed structures (which do not exist in the naive sense that $V$ could be made non-singular). By means of a standard Serre-Siegel argument, one can easily show 
2.11. Proposition. Let $(X, V)$ be a directed variety $(X, V)$ and $F$ be an invertible sheaf on $X$. The following properties are equivalent:

(a) there exists a constant $c>0$ and $m_{0}>0$ such that $h^{0}\left(X, K_{V}^{[m]} \otimes F^{\otimes m}\right) \geqslant c m^{n}$ for $m \geqslant m_{0}$, where $n=\operatorname{dim} X$;

(b) the space of sections $H^{0}\left(X, K_{V}^{[m]} \otimes F^{\otimes m}\right)$ provides a generic embedding of $X$ in projective space for sufficiently large $m$;

(c) there exists $m>0$ and a log-resolution $\mu_{m}: \widehat{X}_{m} \rightarrow X$ of $K_{V}^{[m]}$ such that $\mu_{m}^{*}\left(K_{V}^{[m]} \otimes F^{\otimes m}\right)$ is a big invertible sheaf on $\widehat{X}_{m}$;

(d) there exists $m>0$, a modification $\widetilde{\mu}_{m}:\left(\widetilde{X}_{m}, \widetilde{V}_{m}\right) \rightarrow(X, V)$ and a log-resolution $\mu_{m}^{\prime}: \widehat{X}_{m} \rightarrow \widetilde{X}$ of ${ }^{b} K_{\widetilde{V}_{m}}^{[m]}$ such that $\mu_{m}^{* *}\left({ }^{b} K_{\widetilde{V}_{m}}^{[m]} \otimes \widetilde{\mu}_{m}^{*} F^{\otimes m}\right)$ is a big invertible sheaf on $\widehat{X}_{m}$.

We will express any of these equivalent properties by saying that the twisted pluricanonical sheaf sequence $K_{V}^{\bullet} \otimes F^{\bullet}$ is big.

In the special case $F=\mathcal{O}_{X}$, we introduce

2.12. Definition. We say that $(X, V)$ is of general type if $K_{V}^{\bullet}$ is big.

\subsection{Remarks.}

(a) At this point, it is important to stress the difference between "our" canonical sheaf $K_{V}$, and the sheaf $\mathcal{L}_{V}$, which is considered by some experts as "the canonical sheaf of the foliation" defined by $V$, in the integrable case. Notice that $\mathcal{L}_{V}$ can also be obtained as the direct image $\mathcal{L}_{V}=i_{*} \mathcal{O}\left(\operatorname{det} V^{*}\right)$ associated with the injection $i: X \backslash \operatorname{Sing}(V) \hookrightarrow X$. The discrepancy already occurs with the rank 1 linear space $V \subset T_{\mathbb{P}_{\mathbb{C}}^{n}}$ consisting at each point $z \neq 0$ of the tangent to the line (0z) (so that necessarily $V_{0}=T_{\mathbb{P}_{\mathbb{C}}^{n}, 0}$ ). As a sheaf (and not as a linear space), $i_{*} \mathcal{O}(V)$ is the invertible sheaf generated by the vector field $\xi=\sum z_{j} \partial / \partial z_{j}$ on the affine open set $\mathbb{C}^{n} \subset \mathbb{P}_{\mathbb{C}}^{n}$, and therefore $\mathcal{L}_{V}:=i_{*} \mathcal{O}\left(V^{*}\right)$ is generated over $\mathbb{C}^{n}$ by the unique 1 -form $u$ such that $u(\xi)=1$. Since $\xi$ vanishes at 0 , the generator $u$ is unbounded with respect to a smooth metric $h_{0}$ on $T_{\mathbb{P}_{\mathbb{C}}^{n}}$, and it is easily seen that $K_{V}$ is the non-invertible sheaf $K_{V}=\mathcal{L}_{V} \otimes \mathfrak{m}_{\mathbb{P}_{\mathbb{C}}^{n}, 0}$. We can make it invertible by considering the blow-up $\mu: \widetilde{X} \rightarrow X$ of $X=\mathbb{P}_{\mathbb{C}}^{n}$ at 0 , so that $\mu^{*} K_{V}$ is isomorphic to $\mu^{*} \mathcal{L}_{V} \otimes \mathcal{O}_{\widetilde{X}}(-E)$ where $E$ is the exceptional divisor. The integral curves $C$ of $V$ are of course lines through 0 , and when a standard parametrization is used, their derivatives do not vanish at 0 , while the sections of $i_{*} \mathcal{O}(V)$ do - a first sign that $i_{*} \mathcal{O}(V)$ and $i_{*} \mathcal{O}\left(V^{*}\right)$ are the wrong objects to consider.

(b) When $V$ is of rank 1 , we get a foliation by curves on $X$. If $(X, V)$ is of general type (i.e. $K_{V}^{\bullet}$ is big), we will see in Prop. 4.9 that almost all leaves of $V$ are hyperbolic, i.e. covered by the unit disk. This would not be true if $K_{V}^{\bullet}$ was replaced by $\mathcal{L}_{V}$, In fact, the examples of pencils of conics or cubic curves in $\mathbb{P}^{2}$ already produce this phenomenon, as we have seen in the introduction, right after the generalized GGL conjecture 0.4. For this second reason, we believe that $K_{V}^{\bullet}$ is a more appropriate concept of "canonical sheaf" than $\mathcal{L}_{V}$ is.

(c) When $\operatorname{dim} X=2$, a singularity of a (rank 1) foliation $V$ is said to be simple if the linear part of the local vector field generating $\mathcal{O}(V)$ has two distinct eigenvalues $\lambda \neq 0, \mu \neq 0$ such that the quotient $\lambda / \mu$ is not a positive rational number. Seidenberg's theorem [Sei68] says there always exists a composition of blow-ups $\mu: \widehat{X} \rightarrow X$ such that $\widehat{V}=\mu^{*} V$ only has simple singularities.

It is easy to check that the inductive limit canonical sheaf $K_{V}^{[m]}=\mu_{*}\left({ }^{b} K_{\widehat{V}}^{[m]}\right)$ is reached whenever $\widehat{V}=\mu^{*} V$ has simple singularities.

\section{Algebraic hyperbolicity}

In the case of projective algebraic varieties, hyperbolicity is expected to be related to other properties of a more algebraic nature. Theorem 3.1 below is a first step in this direction. 
3.1. Theorem. Let $(X, V)$ be a compact complex directed manifold and let $\sum \omega_{j k} d z_{j} \otimes d \bar{z}_{k}$ be a Hermitian metric on $T_{X}$, with associated positive $(1,1)$-form $\omega=\frac{i}{2} \sum \omega_{j k} d z_{j} \wedge d \bar{z}_{k}$. Consider the following three properties, which may or not be satisfied by $(X, V)$ :

(i) $(X, V)$ is hyperbolic.

(ii) There exists $\varepsilon>0$ such that every compact irreducible curve $C \subset X$ tangent to $V$ satisfies

$$
-\chi(\bar{C})=2 g(\bar{C})-2 \geqslant \varepsilon \operatorname{deg}_{\omega}(C),
$$

where $\operatorname{deg}_{\omega}(C)=\int_{C} \omega$, and where $g(\bar{C})$ is the genus of the normalization $\bar{C}$ of $C$ and $\chi(\bar{C})$ its Euler characteristic (the degree coincides with the usual concept of degree if $X$ is projective, embedded in $\mathbb{P}^{N}$ via a very ample line bundle $A$, and $\omega=\Theta_{A, h_{A}}>0$; such an estimate is of course independent of the choice of $\omega$, provided that $\varepsilon$ is changed accordingly.)

(iii) There does not exist any non-constant holomorphic map $\Phi: Z \rightarrow X$ from an abelian variety $Z$ to $X$ such that $\Phi_{*}\left(T_{Z}\right) \subset V$.

Then (i) $\Rightarrow$ (ii) $\Rightarrow$ (iii).

Proof. (i) $\Rightarrow$ (ii). If $(X, V)$ is hyperbolic, there is a constant $\varepsilon_{0}>0$ such that $\mathbf{k}_{(X, V)}(\xi) \geqslant \varepsilon_{0}\|\xi\|_{\omega}$ for all $\xi \in V$. Now, let $C \subset X$ be a compact irreducible curve tangent to $V$ and let $\nu: \bar{C} \rightarrow C$ be its normalization. As $(X, V)$ is hyperbolic, $\bar{C}$ cannot be a rational or elliptic curve. Hence $\bar{C}$ admits the disk as its universal covering $\rho: \Delta \rightarrow \bar{C}$.

The Kobayashi-Royden metric $\mathbf{k}_{\Delta}$ is the Finsler metric $|d z| /\left(1-|z|^{2}\right)$ associated with the Poincaré metric $|d z|^{2} /\left(1-|z|^{2}\right)^{2}$ on $\Delta$, and $\mathbf{k}_{\bar{C}}$ is such that $\rho^{*} \mathbf{k}_{\bar{C}}=\mathbf{k}_{\Delta}$. In other words, the metric $\mathbf{k}_{\bar{C}}$ is induced by the unique Hermitian metric on $\bar{C}$ of constant Gaussian curvature -4 . If $\sigma_{\Delta}=$ $\frac{i}{2} d z \wedge d \bar{z} /\left(1-|z|^{2}\right)^{2}$ and $\sigma_{\bar{C}}$ are the corresponding area measures, the Gauss-Bonnet formula (integral of the curvature $=2 \pi \chi(\bar{C}))$ yields

$$
\int_{\bar{C}} d \sigma_{\bar{C}}=-\frac{1}{4} \int_{\bar{C}} \operatorname{curv}\left(\mathbf{k}_{\bar{C}}\right)=-\frac{\pi}{2} \chi(\bar{C})
$$

On the other hand, if $j: C \rightarrow X$ is the inclusion, the monotonicity property (2.2) applied to the holomorphic map $j \circ \nu: \bar{C} \rightarrow X$ shows that

$$
\mathbf{k}_{\bar{C}}(t) \geqslant \mathbf{k}_{(X, V)}\left((j \circ \nu)_{*} t\right) \geqslant \varepsilon_{0}\left\|(j \circ \nu)_{*} t\right\|_{\omega}, \quad \forall t \in T_{\bar{C}} .
$$

From this, we infer $d \sigma_{\bar{C}} \geqslant \varepsilon_{0}^{2}(j \circ \nu)^{*} \omega$, thus

$$
-\frac{\pi}{2} \chi(\bar{C})=\int_{\bar{C}} d \sigma_{\bar{C}} \geqslant \varepsilon_{0}^{2} \int_{\bar{C}}(j \circ \nu)^{*} \omega=\varepsilon_{0}^{2} \int_{C} \omega .
$$

Property (ii) follows with $\varepsilon=2 \varepsilon_{0}^{2} / \pi$.

(ii) $\Rightarrow$ (iii). First observe that (ii) excludes the existence of elliptic and rational curves tangent to $V$. Assume that there is a non-constant holomorphic map $\Phi: Z \rightarrow X$ from an abelian variety $Z$ to $X$ such that $\Phi_{*}\left(T_{Z}\right) \subset V$. We must have $\operatorname{dim} \Phi(Z) \geqslant 2$, otherwise $\Phi(Z)$ would be a curve covered by images of holomorphic maps $\mathbb{C} \rightarrow \Phi(Z)$, and so $\Phi(Z)$ would be elliptic or rational, contradiction. Select a sufficiently general curve $\Gamma$ in $Z$ (e.g., a curve obtained as an intersection of very generic divisors in a given very ample linear system $|L|$ in $Z$ ). Then all isogenies $u_{m}: Z \rightarrow Z, s \mapsto m s$ map $\Gamma$ in a $1: 1$ way to curves $u_{m}(\Gamma) \subset Z$, except maybe for finitely many double points of $u_{m}(\Gamma)$ when $\operatorname{dim} Z=2$ : we leave this as an exercise to the reader, using Bertini type arguments). It follows that the normalization of $u_{m}(\Gamma)$ is isomorphic to $\Gamma$. If $\Gamma$ is general enough and $\tau_{a}: Z \rightarrow Z$, $w \mapsto w+a$ denote translations of $Z$, similar arguments show that for general $a \in Z$ the images

$$
C_{m, a}:=\Phi\left(\tau_{a}\left(u_{m}(\Gamma)\right)\right) \subset X
$$


are also generically $1: 1$ images of $\Gamma$, thus $\bar{C}_{m, a} \simeq \Gamma$ and $g\left(\bar{C}_{m, a}\right)=g(\Gamma)$. We claim that on average $C_{m, a}$ has degree $\geqslant$ Const $m^{2}$. In fact, if $\mu$ is the translation invariant probability measure on $Z$

$$
\int_{C_{m, a}} \omega=\int_{\Gamma} u_{m}^{*}\left(\tau_{a}^{*} \Phi^{*} \omega\right), \quad \text { and hence } \int_{a \in Z}\left(\int_{C_{m, a}} \omega\right) d \mu(a)=\int_{\Gamma} u_{m}^{*} \beta
$$

where $\beta=\int_{a \in Z}\left(\tau_{a}^{*} \Phi^{*} \omega\right) d \mu(a)$ is a translation invariant $(1,1)$-form on $Z$. Therefore $\beta$ is a constant coefficient $(1,1)$-form, so $u_{m}^{*} \beta=m^{2} \beta$ and the right hand side is $\mathrm{cm}^{2}$ with $c=\int_{\Gamma} \beta>0$. For a suitable choice of $a_{m} \in Z$, we have $\operatorname{deg}_{\omega} C_{m, a_{m}} \geqslant c m^{2}$ and $\left(2 g\left(\bar{C}_{m, a_{m}}\right)-2\right) / \operatorname{deg}_{\omega}\left(C_{m, a_{m}}\right) \rightarrow 0$, contradiction.

3.2. Definition. We say that a projective directed manifold $(X, V)$ is "algebraically hyperbolic" if it satisfies Property 3.1 (ii), namely, if there exists $\varepsilon>0$ such that every algebraic curve $C \subset X$ tangent to $V$ satisfies

$$
2 g(\bar{C})-2 \geqslant \varepsilon \operatorname{deg}_{\omega}(C) .
$$

A nice feature of algebraic hyperbolicity is that it satisfies an algebraic analogue of the openness property.

3.3. Proposition. Let $(\mathcal{X}, \mathcal{V}) \rightarrow S$ be an algebraic family of projective algebraic directed manifolds (given by a projective morphism $\mathcal{X} \rightarrow S$ ). Then the set of $t \in S$ such that the fiber $\left(X_{t}, V_{t}\right)$ is algebraically hyperbolic is open with respect to the "countable Zariski topology" of $S$ (by definition, this is the topology for which closed sets are countable unions of algebraic sets).

Proof. After replacing $S$ by a Zariski open subset, we may assume that the total space $\mathcal{X}$ itself is quasi-projective. Let $\omega$ be the Kähler metric on $\mathcal{X}$ obtained by pulling back the Fubini-Study metric via an embedding in a projective space. If the integers $d>0, g \geqslant 0$ are fixed, the set $A_{d, g}$ of $t \in S$ such that $X_{t}$ contains an algebraic 1-cycle $C=\sum m_{j} C_{j}$ tangent to $V_{t}$ with $\operatorname{deg}_{\omega}(C)=d$ and $g(\bar{C})=\sum m_{j} g\left(\bar{C}_{j}\right) \leqslant g$ is a closed algebraic subset of $S$ (this follows from the existence of a relative cycle space of curves of given degree, and from the fact that the geometric genus is Zariski lower semi-continuous). Now, the set of non algebraically hyperbolic fibers is by definition

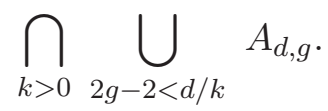

This concludes the proof (of course, one has to know that the countable Zariski topology is actually a topology, namely that the class of countable unions of algebraic sets is stable under arbitrary intersections; this can be easily checked by an induction on dimension).

3.4. Remark. More explicit versions of the openness property have been dealt with in the literature. H. Clemens ([Cle86] and [CKM88]) has shown that on a very generic surface of degree $d \geqslant 5$ in $\mathbb{P}^{3}$, the curves of type $(d, k)$ are of genus $g>k d(d-5) / 2$ (recall that a very generic surface $X \subset \mathbb{P}^{3}$ of degree $\geqslant 4$ has Picard group generated by $\mathcal{O}_{X}(1)$ thanks to the Noether-Lefschetz theorem; thus any curve on the surface is a complete intersection with another hypersurface of degree $k$; such a curve is said to be of type $(d, k)$; genericity is taken here in the sense of the countable Zariski topology). Improving on this result of Clemens, Geng Xu [Xu94] proved that every curve contained in a very generic surface of degree $d \geqslant 5$ satisfies the sharp bound $g \geqslant d(d-3) / 2-2$. In April 2018, I. Coskun and E. Riedl improved the above bounds, and got the more precise bound $g \geqslant 1+(d k(d-5)+k) / 2$; this result actually shows that a very generic surface of degree $d \geqslant 5$ is algebraically hyperbolic in the sense of Definition 3.2. In higher dimension, L. Ein ([Ein88], [Ein91]) proved that every subvariety of a very generic hypersurface $X \subset \mathbb{P}^{n+1}$ of degree $d \geqslant 2 n+1(n \geqslant 2)$, is of general type. This was reproved by a simple efficient technique by C. Voisin in [Voi96], along with other improvements.

3.5. Remark. In view of Proposition 1.10, it would be interesting to know whether algebraic hyperbolicity is open with respect to the Euclidean topology; still more interesting would be 
to know whether Kobayashi hyperbolicity is open for the countable Zariski topology (of course, both properties would follow immediately if one knew that algebraic hyperbolicity and Kobayashi hyperbolicity coincide, but they seem otherwise highly non-trivial to establish). The latter openness property has raised an important amount of work around the following more particular question: is a (very) generic hypersurface $X \subset \mathbb{P}^{n+1}$ of degree $d$ large enough (say $d \geqslant 2 n+1$ ) Kobayashi hyperbolic? Again, "very generic" is to be taken here in the sense of the countable Zariski topology. Brody-Green [BrGr77] and Nadel [Nad89] produced examples of hyperbolic surfaces in $\mathbb{P}^{3}$ for all degrees $d \geqslant 50$, and Masuda-Noguchi [MaNo96] gave examples of such hypersurfaces in $\mathbb{P}^{n}$ for arbitrary $n \geqslant 2$, of degree $d \geqslant d_{0}(n)$ large enough. The hyperbolicity of complements $\mathbb{P}^{n} \backslash H$ of generic divisors may be inferred from the compact case; in fact if $H=\left\{P\left(z_{0}, \ldots, z_{n}\right)=0\right\}$ is a smooth generic divisor of degree $d$, one may look at the hypersurface

$$
X=\left\{z_{n+1}^{d}=P\left(z_{0}, \ldots, z_{n}\right)\right\} \subset \mathbb{P}^{n+1}
$$

which is a cyclic $d: 1$ covering of $\mathbb{P}^{n}$. Since any holomorphic map $f: \mathbb{C} \rightarrow \mathbb{P}^{n} \backslash H$ can be lifted to $X$, it is clear that the hyperbolicity of $X$ would imply the hyperbolicity of $\mathbb{P}^{n} \backslash H$. The hyperbolicity of complements of divisors in $\mathbb{P}^{n}$ has been investigated by many authors. In the case $n=2$, Huynh, $\mathrm{Vu}$ and Xie [HVX17, Theorem 1.2] have announced that $\mathbb{P}^{2} \backslash C$ is hyperbolic for a very general curve $C$ of degree $d \geqslant 11$ (and that a very general surface $X \subset \mathbb{P}^{3}$ of degree $d \geqslant 15$ is hyperbolic, [HVX17, Theorem 1.5]). The reader can also consult [CFZ17, §4] for more details and references in these directions.

In the "absolute case" $V=T_{X}$, it seems reasonable to expect that Properties 3.1 (i), (ii) are equivalent, i.e. that Kobayashi and algebraic hyperbolicity coincide. However, it was observed by Serge Cantat [Can00] that Property 3.1 (iii) is not sufficient to imply the hyperbolicity of $X$, at least when $X$ is a general complex surface: a general (non-algebraic) K3 surface is known to have no elliptic curves and does not admit either any surjective map from an abelian variety; however such a surface is not Kobayashi hyperbolic. We are uncertain about the sufficiency of Property 3.1 (iii) when $X$ is assumed to be projective.

\section{The Ahlfors-Schwarz lemma for metrics of negative Curvature}

One of the most basic ideas is that hyperbolicity should somehow be related with suitable negativity properties of the curvature. For instance, it is a standard fact already observed in Kobayashi [Kob70] that the negativity of $T_{X}$ (or the ampleness of $T_{X}^{*}$ ) implies the hyperbolicity of $X$. There are many ways of improving or generalizing this result. We present here a few simple examples of such generalizations.

\section{A. Exploiting Curvature via potential theory}

If $(V, h)$ is a holomorphic vector bundle equipped with a smooth Hermitian metric, we denote by $\nabla_{h}=\nabla_{h}^{\prime}+\nabla_{h}^{\prime \prime}$ the associated Chern connection and by $\Theta_{V, h}=\frac{i}{2 \pi} \nabla_{h}^{2}$ its Chern curvature tensor.

4.1. Proposition. Let $(X, V)$ be a compact directed manifold. Assume that $V$ is non-singular and that $V^{*}$ is ample. Then $(X, V)$ is hyperbolic.

Proof (from an original idea of [Kob75]). Recall that a vector bundle $E$ is said to be ample if $S^{m} E$ has enough global sections $\sigma_{1}, \ldots, \sigma_{N}$ so as to generate 1-jets of sections at any point, when $m$ is large. One obtains a Finsler metric $N$ on $E^{*}$ by putting

$$
N(\xi)=\left(\sum_{1 \leqslant j \leqslant N}\left|\sigma_{j}(x) \cdot \xi^{m}\right|^{2}\right)^{1 / 2 m}, \quad \xi \in E_{x}^{*},
$$

and $N$ is then a strictly plurisubharmonic function on the total space of $E^{*}$ minus the zero section (in other words, the line bundle $\mathcal{O}_{P\left(E^{*}\right)}(1)$ has a metric of positive curvature). By the ampleness assumption on $V^{*}$, we thus have a Finsler metric $N$ on $V$ which is strictly plurisubharmonic outside the zero section. By the Brody lemma, if $(X, V)$ is not hyperbolic, there is an entire curve 
$g: \mathbb{C} \rightarrow X$ tangent to $V$ such that $\sup _{\mathbb{C}}\left\|g^{\prime}\right\|_{\omega} \leqslant 1$ for some given Hermitian metric $\omega$ on $X$. Then $N\left(g^{\prime}\right)$ is a bounded subharmonic function on $\mathbb{C}$ which is strictly subharmonic on $\left\{g^{\prime} \neq 0\right\}$. This is a contradiction, for any bounded subharmonic function on $\mathbb{C}$ must be constant.

\section{B. AhlFors-Schwarz LEMma}

Proposition 4.1 can be generalized a little bit further by means of the Ahlfors-Schwarz lemma (see e.g. [Lang87]; we refer to [Dem95] for the generalized version presented here; the proof is merely an application of the maximum principle plus a regularization argument).

4.2. Ahlfors-Schwarz lemma. Let $\gamma(t)=\gamma_{0}(t) i d t \wedge d \bar{t}$ be a Hermitian metric on $\Delta_{R}$ where $\log \gamma_{0}$ is a subharmonic function such that $i \partial \bar{\partial} \log \gamma_{0}(t) \geqslant A \gamma(t)$ in the sense of currents, for some positive constant $A$. Then $\gamma$ can be compared with the Poincaré metric of $\Delta_{R}$ as follows:

$$
\gamma(t) \leqslant \frac{2}{A} \frac{R^{-2}|d t|^{2}}{\left(1-|t|^{2} / R^{2}\right)^{2}}
$$

More generally, let $\gamma=i \sum \gamma_{j k} d t_{j} \wedge d \bar{t}_{k}$ be an almost everywhere positive Hermitian form on the ball $B(0, R) \subset \mathbb{C}^{p}$, such that $-\operatorname{Ricci}(\gamma):=i \partial \bar{\partial} \log \operatorname{det}(\gamma) \geqslant A \gamma$ in the sense of currents, for some constant $A>0$ (this means in particular that $\operatorname{det}(\gamma)=\operatorname{det}\left(\gamma_{j k}\right)$ is such that $\log \operatorname{det}(\gamma)$ is plurisubharmonic). Then the $\gamma$-volume form is controlled by the Poincaré volume form :

$$
\operatorname{det}(\gamma) \leqslant\left(\frac{p+1}{A R^{2}}\right)^{p} \frac{1}{\left(1-|t|^{2} / R^{2}\right)^{p+1}} .
$$

\section{C. Applichtions of the Ahlfors-Schwarz lemma to hyperbolicity}

Let $(X, V)$ be a projective directed variety. We assume throughout this subsection that $X$ is non-singular.

4.3. Proposition. Assume that $V$ itself is non-singular and that the dual bundle $V^{*}$ is "very big" in the following sense: there exists an ample line bundle $L$ and a sufficiently large integer $m$ such that the global sections in $H^{0}\left(X, S^{m} V^{*} \otimes L^{-1}\right)$ generate all fibers over $X \backslash Y$, for some analytic subset $Y \subsetneq X$. Then all entire curves $f: \mathbb{C} \rightarrow X$ tangent to $V$ satisfy $f(\mathbb{C}) \subset Y$.

Proof. Let $\sigma_{1}, \ldots, \sigma_{N} \in H^{0}\left(X, S^{m} V^{*} \otimes L^{-1}\right)$ be a basis of sections generating $S^{m} V^{*} \otimes L^{-1}$ over $X \backslash Y$. If $f: \mathbb{C} \rightarrow X$ is tangent to $V$, we define a semi-positive Hermitian form $\gamma(t)=\gamma_{0}(t)|d t|^{2}$ on $\mathbb{C}$ by putting

$$
\gamma_{0}(t)=\sum\left\|\sigma_{j}(f(t)) \cdot f^{\prime}(t)^{m}\right\|_{L^{-1}}^{2 / m}
$$

where \|\|$_{L}$ denotes a Hermitian metric with positive curvature on $L$. If $f(\mathbb{C}) \not \subset Y$, the form $\gamma$ is not identically 0 and we then find

$$
i \partial \bar{\partial} \log \gamma_{0} \geqslant \frac{2 \pi}{m} f^{*} \Theta_{L}
$$

where $\Theta_{L}$ is the curvature form. The positivity assumption combined with an obvious homogeneity argument yield

$$
\frac{2 \pi}{m} f^{*} \Theta_{L} \geqslant \varepsilon\left\|f^{\prime}(t)\right\|_{\omega}^{2}|d t|^{2} \geqslant \varepsilon^{\prime} \gamma(t)
$$

for any given Hermitian metric $\omega$ on $X$. Now, for any $t_{0}$ with $\gamma_{0}\left(t_{0}\right)>0$, the Ahlfors-Schwarz lemma shows that $f$ can only exist on a disk $D\left(t_{0}, R\right)$ such that $\gamma_{0}\left(t_{0}\right) \leqslant \frac{2}{\varepsilon^{\prime}} R^{-2}$, contradiction.

There are similar results for $p$-measure hyperbolicity, see e.g. [Carl72] and [Nog77b]:

4.4. Proposition. Assume that $V$ is non-singular and that $\Lambda^{p} V^{*}$ is ample. Then $(X, V)$ is infinitesimally p-measure hyperbolic. More generally, assume that $\Lambda^{p} V^{*}$ is very big with base locus contained in $Y \subsetneq X$ (see Proposition 3.3). Then $\mathbf{e}^{p}$ is non-degenerate over $X \backslash Y$. 
Proof. By the ampleness assumption, there is a smooth Finsler metric $N$ on $\Lambda^{p} V$ which is strictly plurisubharmonic outside the zero section. We select also a Hermitian metric $\omega$ on $X$. For any holomorphic map $f: \mathbb{B}_{p} \rightarrow X$ we define a semi-positive Hermitian metric $\widetilde{\gamma}$ on $\mathbb{B}_{p}$ by putting $\widetilde{\gamma}=f^{*} \omega$. Since $\omega$ need not have any good curvature estimate, we introduce the function $\delta(t)=$ $N_{f(t)}\left(\Lambda^{p} f^{\prime}(t) \cdot \tau_{0}\right)$, where $\tau_{0}=\partial / \partial t_{1} \wedge \cdots \wedge \partial / \partial t_{p}$, and select a metric $\gamma=\lambda \widetilde{\gamma}$ conformal to $\widetilde{\gamma}$ such that $\operatorname{det}(\gamma)=\delta$. Then $\lambda^{p}$ is equal to the ratio $N / \Lambda^{p} \omega$ on the element $\Lambda^{p} f^{\prime}(t) \cdot \tau_{0} \in \Lambda^{p} V_{f(t)}$. Since $X$ is compact, it is clear that the conformal factor $\lambda$ is bounded by an absolute constant independent of $f$. From the curvature assumption we then get

$$
i \partial \bar{\partial} \log \operatorname{det}(\gamma)=i \partial \bar{\partial} \log \delta \geqslant\left(f, \Lambda^{p} f^{\prime}\right)^{*}(i \partial \bar{\partial} \log N) \geqslant \varepsilon f^{*} \omega \geqslant \varepsilon^{\prime} \gamma
$$

By the Ahlfors-Schwarz lemma we infer that $\operatorname{det}(\gamma(0)) \leqslant C$ for some constant $C$, i.e., $N_{f(0)}\left(\Lambda^{p} f^{\prime}(0)\right.$. $\left.\tau_{0}\right) \leqslant C^{\prime}$. This means that the Kobayashi-Eisenman pseudometric $\mathbf{e}_{(X, V)}^{p}$ is positive definite everywhere and uniformly bounded from below. In the case $\Lambda^{p} V^{*}$ is very big with base locus $Y$, we use essentially the same arguments, but we then only have $N$ being positive definite on $X \backslash Y$.

4.5. Corollary ([Gri71], KobO71]). If $X$ is a projective variety of general type, the KobayashiEisenmann volume form $\mathbf{e}^{n}, n=\operatorname{dim} X$, can degenerate only along a proper algebraic set $Y \subsetneq X$.

The converse of Corollary 4.5 is expected to be true, namely, the generic non-degeneracy of $\mathbf{e}^{n}$ should imply that $X$ is of general type; this is only known for surfaces (see [GrGr80] and [MoMu82]):

4.6. General Type Conjecture (Green-Griffiths [GrGr80]). A projective algebraic variety $X$ is measure hyperbolic (i.e. $\mathbf{e}^{n}$ degenerates only along a proper algebraic subvariety) if and only if $X$ is of general type.

An essential step in the proof of the necessity of having general type subvarieties would be to show that manifolds of Kodaira dimension 0 (say, Calabi-Yau manifolds and holomorphic symplectic manifolds, all of which have $c_{1}(X)=0$ ) are not measure hyperbolic, e.g. by exhibiting enough families of curves $C_{s, \ell}$ covering $X$ such that $\left(2 g\left(\bar{C}_{s, \ell}\right)-2\right) / \operatorname{deg}\left(C_{s, \ell}\right) \rightarrow 0$.

4.7. Conjectural corollary (Lang). A projective algebraic variety $X$ is hyperbolic if and only if all its algebraic subvarieties (including $X$ itself) are of general type.

4.8. Remark. The GGL conjecture implies the "if" part of 4.7, and the General Type Conjecture 4.6 implies the "only if" part of 4.7. In fact if the GGL conjecture holds and every subvariety $Y$ of $X$ is of general type, then it is easy to infer that every entire curve $f: \mathbb{C} \rightarrow X$ has to be constant by induction on $\operatorname{dim} X$, because in fact $f$ maps $\mathbb{C}$ to a certain subvariety $Y \subsetneq X$. Therefore $X$ is hyperbolic. Conversely, if Conjecture 4.6 holds and $X$ has a certain subvariety $Y$ which is not of general type, then $Y$ is not measure hyperbolic. However Proposition 2.4 shows that hyperbolicity implies measure hyperbolicity. Therefore $Y$ is not hyperbolic and so $X$ itself is not hyperbolic either.

We end this section by another easy application of the Ahlfors-Schwarz lemma for the case of rank 1 (possibly singular) foliations.

4.9. Proposition. Let $(X, V)$ be a projective directed manifold. Assume that $V$ is of rank 1 and that $K_{V}^{\bullet}$ is big. Then $S$ be the union of the singular set $\operatorname{Sing}(V)$ and of the base locus of $K_{V}^{\bullet}$ (namely the intersection of the images $\mu_{m}\left(B_{m}\right)$ of the base loci $B_{m}$ of the invertible sheaves $\mu_{m}^{*} K_{V}^{[m]}, m>0$, obtained by taking log-resolutions). Then $\operatorname{ECL}(X, V) \subset S$, in other words, all non-hyperbolic leaves of $V$ are contained in $S$.

Proof. By Prop. 2.11 (d), we can take a blow-up $\widetilde{\mu}_{m}: \widetilde{X}_{m} \rightarrow X$ and a log-resolution $\mu_{m}^{\prime}: \widehat{X}_{m} \rightarrow \widetilde{X}_{m}$ such that $F_{m}=\mu_{m}^{\prime *}\left({ }^{b} K_{\widetilde{V}_{m}}^{[m]}\right)$ is a big invertible sheaf. This means that (after possibly increasing $m$ ) we can find sections $\sigma_{1}, \ldots \sigma_{N} \in H^{0}\left(\widehat{X}_{m}, F_{m}\right)$ that define a (singular) Hermitian metric with strictly positive curvature on $F_{m}$, cf. Def. 8.1 below. Now, for every entire curve $f:\left(\mathbb{C}, T_{\mathbb{C}}\right) \rightarrow(X, V)$ not contained in $S$, we can choose $m$ and a lifting $\widetilde{f}:\left(\mathbb{C}, T_{\mathbb{C}}\right) \rightarrow(\widetilde{X}, \widetilde{V})$ such that $\widetilde{f}(\mathbb{C})$ is not 
contained in the base locus of our sections. Again, we can define a semi-positive Hermitian form $\gamma(t)=\gamma_{0}(t)|d t|^{2}$ on $\mathbb{C}$ by putting

$$
\gamma_{0}(t)=\sum\left\|\sigma_{j}(f(t)) \cdot f^{\prime}(t)^{m}\right\|_{L^{-1}}^{2 / m} .
$$

Then $\gamma$ is not identically zero and we have $i \partial \bar{\partial} \log \gamma_{0} \geqslant \varepsilon \gamma$ by the strict positivity of the curvature. One should also notice that $\gamma_{0}$ is locally bounded from above by the assumption that the $\sigma_{j}$ 's come from locally bounded sections on $\widetilde{X}_{m}$. This contradicts the Ahlfors-Schwarz lemma, and thus it cannot happen that $f(\mathbb{C}) \not \subset S$.

\section{Projectivization of a DiRected manifold}

\section{A. The 1-JeT FUNCTOR}

The basic idea is to introduce a functorial process which produces a new complex directed manifold $(\widetilde{X}, \widetilde{V})$ from a given one $(X, V)$. The new structure $(\widetilde{X}, \widetilde{V})$ plays the role of a space of 1-jets over $X$. Fisrt assume that $V$ is non-singular. We let

$$
\widetilde{X}=P(V), \quad \widetilde{V} \subset T_{\widetilde{X}}
$$

be the projectivized bundle of lines of $V$, together with a subbundle $\widetilde{V}$ of $T_{\widetilde{X}}$ defined as follows: for every point $(x,[v]) \in \widetilde{X}$ associated with a vector $v \in V_{x} \backslash\{0\}$,

$$
\widetilde{V}_{(x,[v])}=\left\{\xi \in T_{\tilde{X},(x,[v])} ; \pi_{*} \xi \in \mathbb{C} v\right\}, \quad \mathbb{C} v \subset V_{x} \subset T_{X, x},
$$

where $\pi: \widetilde{X}=P(V) \rightarrow X$ is the natural projection and $\pi_{*}: T_{\widetilde{X}} \rightarrow \pi^{*} T_{X}$ is its differential. On $\widetilde{X}=P(\underset{V}{V})$ we have a tautological line bundle $\mathcal{O}_{\widetilde{X}}(-1) \subset \pi^{*} V$ such that $\mathcal{O}_{\tilde{X}}(-1)_{(x,[v])}=\mathbb{C} v$. The bundle $\widetilde{V}$ is characterized by the two exact sequences

$$
\begin{aligned}
& 0 \longrightarrow T_{\tilde{X} / X} \longrightarrow \widetilde{V} \stackrel{\pi_{*}}{\longrightarrow} \mathcal{O}_{\widetilde{X}}(-1) \longrightarrow 0, \\
& 0 \longrightarrow \mathcal{O}_{\tilde{X}} \longrightarrow \pi^{*} V \otimes \mathcal{O}_{\widetilde{X}}(1) \longrightarrow T_{\widetilde{X} / X} \longrightarrow 0,
\end{aligned}
$$

where $T_{\widetilde{X} / X}$ denotes the relative tangent bundle of the fibration $\pi: \widetilde{X} \rightarrow X$. The first sequence is a direct consequence of the definition of $\widetilde{V}$, whereas the second is a relative version of the Euler exact sequence describing the tangent bundle of the fibers $P\left(V_{x}\right)$. From these exact sequences we infer

$$
\operatorname{dim} \widetilde{X}=n+r-1, \quad \operatorname{rank} \widetilde{V}=\operatorname{rank} V=r,
$$

and by taking determinants we find $\operatorname{det}\left(T_{\tilde{X} / X}\right)=\pi^{*} \operatorname{det}(V) \otimes \mathcal{O}_{\widetilde{X}}(r)$. Thus

$$
\operatorname{det}(\widetilde{V})=\pi^{*} \operatorname{det}(V) \otimes \mathcal{O}_{\tilde{X}}(r-1) .
$$

By definition, $\pi:(\widetilde{X}, \widetilde{V}) \rightarrow(X, V)$ is a morphism of complex directed manifolds. Clearly, our construction is functorial, i.e., for every morphism of directed manifolds $\Phi:(X, V) \rightarrow(Y, W)$, there is a commutative diagram

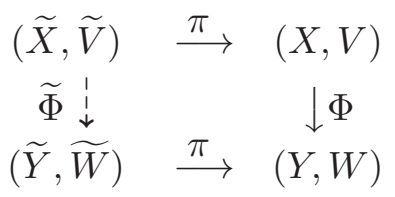

where the left vertical arrow is the meromorphic map $P(V) \rightarrow P(W)$ induced by the differential $\Phi_{*}: V \rightarrow \Phi^{*} W\left(\widetilde{\Phi}\right.$ is actually holomorphic if $\Phi_{*}: V \rightarrow \Phi^{*} W$ is injective). 


\section{B. Lifting of CURVes to the 1-Jet Bundle}

Suppose that we are given a holomorphic curve $f: \Delta_{R} \rightarrow X$ parametrized by the disk $\Delta_{R}$ of centre 0 and radius $R$ in the complex plane, and that $f$ is a tangent curve of the directed manifold, i.e., $f^{\prime}(t) \in V_{f(t)}$ for every $t \in \Delta_{R}$. If $f$ is non-constant, there is a well defined and unique tangent line $\left[f^{\prime}(t)\right] \in P\left(V_{f(t)}\right)$ for every $t$, even at stationary points, and the map

$$
\tilde{f}: \Delta_{R} \rightarrow \tilde{X}, \quad t \mapsto \widetilde{f}(t):=\left(f(t),\left[f^{\prime}(t)\right]\right)
$$

is holomorphic; in fact, at a stationary point $t_{0}$, we can write $f^{\prime}(t)=\left(t-t_{0}\right)^{s} u(t)$ with $s \in \mathbb{N}^{*}$ and $u\left(t_{0}\right) \neq 0$, and we define the tangent line at $t_{0}$ to be $\left[u\left(t_{0}\right)\right]$, so that $\tilde{f}(t)=(f(t),[u(t)])$ near $t_{0}$; even for $t=t_{0}$, we still denote $\left[f^{\prime}\left(t_{0}\right)\right]=\left[u\left(t_{0}\right)\right]$ for simplicity of notation. By definition $f^{\prime}(t) \in \mathcal{O}_{\widetilde{X}}(-1) \widetilde{f}(t)=\mathbb{C} u(t)$, so the derivative $f^{\prime}$ defines a section

$$
f^{\prime}: T_{\Delta_{R}} \rightarrow \widetilde{f}^{*} \mathcal{O}_{\tilde{X}}(-1)
$$

Moreover $\pi \circ \tilde{f}=f$, and thus

$$
\pi_{*} \widetilde{f}^{\prime}(t)=f^{\prime}(t) \in \mathbb{C} u(t) \Longrightarrow \widetilde{f}^{\prime}(t) \in \widetilde{V}_{(f(t), u(t))}=\widetilde{V}_{\tilde{f}(t)}
$$

and we see that $\tilde{f}$ is a tangent trajectory of $(\widetilde{X}, \widetilde{V})$. We say that $\widetilde{f}$ is the canonical lifting of $f$ to $\widetilde{X}$. Conversely, if $g: \Delta_{R} \rightarrow \widetilde{X}$ is a tangent trajectory of $(\widetilde{X}, \widetilde{V})$, then by definition of $\widetilde{V}$ we see that $f=\pi \circ g$ is a tangent trajectory of $(X, V)$ and that $g=\widetilde{f}$ (unless $g$ is contained in a vertical fiber $P\left(V_{x}\right)$, in which case $f$ is constant).

For any point $x_{0} \in X$, there are local coordinates $\left(z_{1}, \ldots, z_{n}\right)$ on a neighborhood $\Omega$ of $x_{0}$ such that the fibers $\left(V_{z}\right)_{z \in \Omega}$ can be defined by linear equations

$$
V_{z}=\left\{\xi=\sum_{1 \leqslant j \leqslant n} \xi_{j} \frac{\partial}{\partial z_{j}} ; \xi_{j}=\sum_{1 \leqslant k \leqslant r} a_{j k}(z) \xi_{k} \text { for } j=r+1, \ldots, n\right\},
$$

where $\left(a_{j k}\right)$ is a holomorphic $(n-r) \times r$ matrix. It follows that a vector $\xi \in V_{z}$ is completely determined by its first $r$ components $\left(\xi_{1}, \ldots, \xi_{r}\right)$, and the affine chart $\xi_{j} \neq 0$ of $P(V)_{\mid \Omega}$ can be described by the coordinate system

$$
\left(z_{1}, \ldots, z_{n} ; \frac{\xi_{1}}{\xi_{j}}, \ldots, \frac{\xi_{j-1}}{\xi_{j}}, \frac{\xi_{j+1}}{\xi_{j}}, \ldots, \frac{\xi_{r}}{\xi_{j}}\right) .
$$

Let $f \simeq\left(f_{1}, \ldots, f_{n}\right)$ be the components of $f$ in the coordinates $\left(z_{1}, \ldots, z_{n}\right)$ (we suppose here $R$ so small that $f\left(\Delta_{R}\right) \subset \Omega$ ). It should be observed that $f$ is uniquely determined by its initial value $x$ and by the first $r$ components $\left(f_{1}, \ldots, f_{r}\right)$. Indeed, as $f^{\prime}(t) \in V_{f(t)}$, we can recover the other components by integrating the system of ordinary differential equations

$$
f_{j}^{\prime}(t)=\sum_{1 \leqslant k \leqslant r} a_{j k}(f(t)) f_{k}^{\prime}(t), \quad j>r,
$$

on a neighborhood of 0 , with initial data $f(0)=x$. We denote by $m=m\left(f, t_{0}\right)$ the multiplicity of $f$ at any point $t_{0} \in \Delta_{R}$, that is, $m\left(f, t_{0}\right)$ is the smallest integer $m \in \mathbb{N}^{*}$ such that $f_{j}^{(m)}\left(t_{0}\right) \neq 0$ for some $j$. By (5.10), we can always suppose $j \in\{1, \ldots, r\}$, for example $f_{r}^{(m)}\left(t_{0}\right) \neq 0$. Then $f^{\prime}(t)=\left(t-t_{0}\right)^{m-1} u(t)$ with $u_{r}\left(t_{0}\right) \neq 0$, and the lifting $\tilde{f}$ is described in the coordinates of the affine chart $\xi_{r} \neq 0$ of $P(V)_{\Gamma \Omega}$ by

$$
\widetilde{f} \simeq\left(f_{1}, \ldots, f_{n} ; \frac{f_{1}^{\prime}}{f_{r}^{\prime}}, \ldots, \frac{f_{r-1}^{\prime}}{f_{r}^{\prime}}\right)
$$

\section{C. Curvature properties of the 1-Jet Bundle}

We end this section with a few curvature computations. Assume that $V$ is non singular and equipped with a smooth Hermitian metric $h$. Denote by $\nabla_{h}=\nabla_{h}^{\prime}+\nabla_{h}^{\prime \prime}$ the associated Chern 
connection and by $\Theta_{V, h}=\frac{i}{2 \pi} \nabla_{h}^{2}$ its Chern curvature tensor. For every point $x_{0} \in X$, there exists a "normalized" holomorphic frame $\left(e_{\lambda}\right)_{1 \leqslant \lambda \leqslant r}$ on a neighborhood of $x_{0}$, such that

$$
\left\langle e_{\lambda}, e_{\mu}\right\rangle_{h}=\delta_{\lambda \mu}-\sum_{1 \leqslant j, k \leqslant n} c_{j k \lambda \mu} z_{j} \bar{z}_{k}+O\left(|z|^{3}\right),
$$

with respect to any holomorphic coordinate system $\left(z_{1}, \ldots, z_{n}\right)$ centered at $x_{0}$. A computation of $d^{\prime}\left\langle e_{\lambda}, e_{\mu}\right\rangle_{h}=\left\langle\nabla_{h}^{\prime} e_{\lambda}, e_{\mu}\right\rangle_{h}$ and $\nabla_{h}^{2} e_{\lambda}=d^{\prime \prime} \nabla_{h}^{\prime} e_{\lambda}$ then gives

$$
\begin{aligned}
\nabla_{h}^{\prime} e_{\lambda} & =-\sum_{j, k, \mu} c_{j k \lambda \mu} \bar{z}_{k} d z_{j} \otimes e_{\mu}+O\left(|z|^{2}\right), \\
\Theta_{V, h}\left(x_{0}\right) & =\frac{i}{2 \pi} \sum_{j, k, \lambda, \mu} c_{j k \lambda \mu} d z_{j} \wedge d \bar{z}_{k} \otimes e_{\lambda}^{*} \otimes e_{\mu} .
\end{aligned}
$$

The above curvature tensor can also be viewed as a Hermitian form on $T_{X} \otimes V$. In fact, one associates with $\Theta_{V, h}$ the Hermitian form $\left\langle\Theta_{V, h}\right\rangle$ on $T_{X} \otimes V$ defined for all $(\zeta, v) \in T_{X} \times_{X} V$ by

$$
\left\langle\Theta_{V, h}\right\rangle(\zeta \otimes v)=\sum_{1 \leqslant j, k \leqslant n, 1 \leqslant \lambda, \mu \leqslant r} c_{j k \lambda \mu} \zeta_{j} \bar{\zeta}_{k} v_{\lambda} \bar{v}_{\mu}
$$

Let $h_{1}$ be the Hermitian metric on the tautological line bundle $\mathcal{O}_{P(V)}(-1) \subset \pi^{*} V$ induced by the metric $h$ of $V$. We compute the curvature $(1,1)$-form $\Theta_{h_{1}}\left(\mathcal{O}_{P(V)}(-1)\right)$ at an arbitrary point $\left(x_{0},\left[v_{0}\right]\right) \in P(V)$, in terms of $\Theta_{V, h}$. For simplicity, we suppose that the frame $\left(e_{\lambda}\right)_{1 \leqslant \lambda \leqslant r}$ has been chosen in such a way that $\left[e_{r}\left(x_{0}\right)\right]=\left[v_{0}\right] \in P(V)$ and $\left|v_{0}\right|_{h}=1$. We get holomorphic local coordinates $\left(z_{1}, \ldots, z_{n} ; \xi_{1}, \ldots, \xi_{r-1}\right)$ on a neighborhood of $\left(x_{0},\left[v_{0}\right]\right)$ in $P(V)$ by assigning

$$
\left(z_{1}, \ldots, z_{n} ; \xi_{1}, \ldots, \xi_{r-1}\right) \longmapsto\left(z,\left[\xi_{1} e_{1}(z)+\cdots+\xi_{r-1} e_{r-1}(z)+e_{r}(z)\right]\right) \in P(V) .
$$

Then the function

$$
\eta(z, \xi)=\xi_{1} e_{1}(z)+\cdots+\xi_{r-1} e_{r-1}(z)+e_{r}(z)
$$

defines a holomorphic section of $\mathcal{O}_{P(V)}(-1)$ in a neighborhood of $\left(x_{0},\left[v_{0}\right]\right)$. By using the expansion (5.12) for $h$, we find

$$
\begin{aligned}
|\eta|_{h_{1}}^{2}=|\eta|_{h}^{2}=1+|\xi|^{2} & -\sum_{1 \leqslant j, k \leqslant n} c_{j k r r} z_{j} \bar{z}_{k}+O\left((|z|+|\xi|)^{3}\right), \\
\Theta_{h_{1}}\left(\mathcal{O}_{P(V)}(-1)\right)_{\left(x_{0},\left[v_{0}\right]\right)} & =-\frac{i}{2 \pi} \partial \bar{\partial} \log |\eta|_{h_{1}}^{2} \\
& =\frac{i}{2 \pi}\left(\sum_{1 \leqslant j, k \leqslant n} c_{j k r r} d z_{j} \wedge d \bar{z}_{k}-\sum_{1 \leqslant \lambda \leqslant r-1} d \xi_{\lambda} \wedge d \bar{\xi}_{\lambda}\right) .
\end{aligned}
$$

\section{Jets of Curves And Semple Jet Bundles}

\section{A. SEMPle TOWER OF NON-SINGULAR DIRECTED VARIETIES}

Let $X$ be a complex $n$-dimensional manifold. Following ideas of Green-Griffiths [GrGr80], we let $J_{k} X \rightarrow X$ be the bundle of $k$-jets of germs of parametrized curves in $X$, that is, the set of equivalence classes of holomorphic maps $f:(\mathbb{C}, 0) \rightarrow(X, x)$, with the equivalence relation $f \sim g$ if and only if all derivatives $f^{(j)}(0)=g^{(j)}(0)$ coincide for $0 \leqslant j \leqslant k$, when computed in some local coordinate system of $X$ near $x$. The projection map $J_{k} X \rightarrow X$ is simply $f \mapsto f(0)$. If $\left(z_{1}, \ldots, z_{n}\right)$ are local holomorphic coordinates on an open set $\Omega \subset X$, the elements $f$ of any fiber $J_{k} X_{x}, x \in \Omega$, can be seen as $\mathbb{C}^{n}$-valued maps

$$
f=\left(f_{1}, \ldots, f_{n}\right):(\mathbb{C}, 0) \rightarrow \Omega \subset \mathbb{C}^{n},
$$

and they are completely determined by their Taylor expansion of order $k$ at $t=0$

$$
f(t)=x+t f^{\prime}(0)+\frac{t^{2}}{2 !} f^{\prime \prime}(0)+\cdots+\frac{t^{k}}{k !} f^{(k)}(0)+O\left(t^{k+1}\right) .
$$


In these coordinates, the fiber $J_{k} X_{x}$ can thus be identified with the set of $k$-tuples of vectors $\left(\xi_{1}, \ldots, \xi_{k}\right)=\left(f^{\prime}(0), \ldots, f^{(k)}(0)\right) \in\left(\mathbb{C}^{n}\right)^{k}$. It follows that $J_{k} X$ is a holomorphic fiber bundle with typical fiber $\left(\mathbb{C}^{n}\right)^{k}$ over $X$ (however, $J_{k} X$ is not a vector bundle for $k \geqslant 2$, because of the nonlinearity of coordinate changes; see formula (7.2) in $\S 7$ ).

According to the philosophy developed throughout this paper, we describe the concept of jet bundle in the general situation of complex directed manifolds. If $X$ is equipped with a holomorphic subbundle $V \subset T_{X}$, we associate to $V$ a $k$-jet bundle $J_{k} V$ as follows, assuming $V$ non-singular throughout subsection 6.A.

6.1. Definition. Let $(X, V)$ be a complex directed manifold. We define $J_{k} V \rightarrow X$ to be the bundle of $k$-jets of curves $f:(\mathbb{C}, 0) \rightarrow X$ which are tangent to $V$, i.e., such that $f^{\prime}(t) \in V_{f(t)}$ for all $t$ in a neighborhood of 0 , together with the projection map $f \mapsto f(0)$ onto $X$.

It is easy to check that $J_{k} V$ is actually a subbundle of $J_{k} X$. In fact, by using (5.8) and (5.10), we see that the fibers $J_{k} V_{x}$ are parametrized by

$$
\left(\left(f_{1}^{\prime}(0), \ldots, f_{r}^{\prime}(0)\right) ;\left(f_{1}^{\prime \prime}(0), \ldots, f_{r}^{\prime \prime}(0)\right) ; \ldots ;\left(f_{1}^{(k)}(0), \ldots, f_{r}^{(k)}(0)\right)\right) \in\left(\mathbb{C}^{r}\right)^{k}
$$

for all $x \in \Omega$, and hence $J_{k} V$ is a locally trivial $\left(\mathbb{C}^{r}\right)^{k}$-subbundle of $J_{k} X$. Alternatively, we can pick a local holomorphic connection $\nabla$ on $V$ such that for any germs $w=\sum_{1 \leqslant j \leqslant n} w_{j} \frac{\partial}{\partial z_{j}} \in \mathcal{O}\left(T_{X, x}\right)$ and $v=\sum_{1 \leqslant \lambda \leqslant r} v_{\lambda} e_{\lambda} \in \mathcal{O}(V)_{x}$ in a local trivializing frame $\left(e_{1}, \ldots, e_{r}\right)$ of $V_{\uparrow \Omega}$ we have

$$
\nabla_{w} v(x)=\sum_{1 \leqslant j \leqslant n, 1 \leqslant \lambda \leqslant r} w_{j} \frac{\partial v_{\lambda}}{\partial z_{j}} e_{\lambda}(x)+\sum_{1 \leqslant j \leqslant n, 1 \leqslant \lambda, \mu \leqslant r} \Gamma_{j \lambda}^{\mu}(x) w_{j} v_{\lambda} e_{\mu}(x) .
$$

We can of course take the frame obtained from (5.8) by lifting the vector fields $\partial / \partial z_{1}, \ldots, \partial / \partial z_{r}$, and the "trivial connection" given by the zero Christoffel symbols $\Gamma=0$. One then obtains a trivialization $J^{k} V_{\uparrow \Omega} \simeq V_{\uparrow \Omega}^{\oplus k}$ by considering

$$
J_{k} V_{x} \ni f \mapsto\left(\xi_{1}, \xi_{2}, \ldots, \xi_{k}\right)=\left(\nabla f(0), \nabla^{2} f(0), \ldots, \nabla^{k} f(0)\right) \in V_{x}^{\oplus k}
$$

and computing inductively the successive derivatives $\nabla f(t)=f^{\prime}(t)$ and $\nabla^{s} f(t)$ via

$$
\nabla^{s} f=\left(f^{*} \nabla\right)_{d / d t}\left(\nabla^{s-1} f\right)=\sum_{1 \leqslant \lambda \leqslant r} \frac{d}{d t}\left(\nabla^{s-1} f\right)_{\lambda} e_{\lambda}(f)+\sum_{1 \leqslant j \leqslant n, 1 \leqslant \lambda, \mu \leqslant r} \Gamma_{j \lambda}^{\mu}(f) f_{j}^{\prime}\left(\nabla^{s-1} f\right)_{\lambda} e_{\mu}(f) .
$$

This identification depends of course on the choice of $\nabla$ and cannot be defined globally in general (unless we are in the rare situation where $V$ has a global holomorphic connection.

We now describe a convenient process for constructing "projectivized jet bundles", which will later appear as natural quotients of our jet bundles $J_{k} V$ (or rather, as suitable desingularized compactifications of the quotients). Such spaces have already been considered since a long time, at least in the special case $X=\mathbb{P}^{2}, V=T_{\mathbb{P}^{2}}$ (see Gherardelli [Ghe41], Semple [Sem54]), and they have been mostly used as a tool for establishing enumerative formulas dealing with the order of contact of plane curves (see [Coll88], [CoKe94]); the article [ASS97] is also concerned with such generalizations of jet bundles, as well as [LaTh96] by Laksov and Thorup.

We define inductively the projectivized $k$-jet bundle $X_{k}$ (or Semple $k$-jet bundle) and the associated subbundle $V_{k} \subset T_{X_{k}}$ by

$$
\left(X_{0}, V_{0}\right)=(X, V), \quad\left(X_{k}, V_{k}\right)=\left(\widetilde{X}_{k-1}, \widetilde{V}_{k-1}\right) .
$$

In other words, $\left(X_{k}, V_{k}\right)$ is obtained from $(X, V)$ by iterating $k$-times the lifting construction $(X, V) \mapsto(\widetilde{X}, \widetilde{V})$ described in $\S 5$. By $(5.2-5.7)$, we find

$$
\operatorname{dim} X_{k}=n+k(r-1), \quad \operatorname{rank} V_{k}=r,
$$


together with exact sequences

$$
\begin{aligned}
& 0 \longrightarrow T_{X_{k} / X_{k-1}} \longrightarrow V_{k} \stackrel{\left(\pi_{k}\right)_{*}}{\longrightarrow} \mathcal{O}_{X_{k}}(-1) \longrightarrow 0, \\
& 0 \longrightarrow \mathcal{O}_{X_{k}} \longrightarrow \pi_{k}^{*} V_{k-1} \otimes \mathcal{O}_{X_{k}}(1) \longrightarrow T_{X_{k} / X_{k-1}} \longrightarrow 0,
\end{aligned}
$$

where $\pi_{k}$ is the natural projection $\pi_{k}: X_{k} \rightarrow X_{k-1}$ and $\left(\pi_{k}\right)_{*}$ its differential. Formula (5.4) yields

$$
\operatorname{det} V_{k}=\pi_{k}^{*} \operatorname{det} V_{k-1} \otimes \mathcal{O}_{X_{k}}(r-1) \text {. }
$$

Every non-constant tangent trajectory $f: \Delta_{R} \rightarrow X$ of $(X, V)$ lifts to a well defined and unique tangent trajectory $f_{[k]}: \Delta_{R} \rightarrow X_{k}$ of $\left(X_{k}, V_{k}\right)$. Moreover, the derivative $f_{[k-1]}^{\prime}$ gives rise to a section

$$
f_{[k-1]}^{\prime}: T_{\Delta_{R}} \rightarrow f_{[k]}^{*} \mathcal{O}_{X_{k}}(-1) .
$$

In coordinates, one can compute $f_{[k]}$ in terms of its components in the various affine charts (5.9) occurring at each step: we get inductively

$$
f_{[k]}=\left(F_{1}, \ldots, F_{N}\right), \quad f_{[k+1]}=\left(F_{1}, \ldots, F_{N}, \frac{F_{s_{1}}^{\prime}}{F_{s_{r}}^{\prime}}, \ldots, \frac{F_{s_{r-1}}^{\prime}}{F_{s_{r}}^{\prime}}\right)
$$

where $N=n+k(r-1)$ and $\left\{s_{1}, \ldots, s_{r}\right\} \subset\{1, \ldots, N\}$. If $k \geqslant 1,\left\{s_{1}, \ldots, s_{r}\right\}$ contains the last $r-1$ indices of $\{1, \ldots, N\}$ corresponding to the "vertical" components of the projection $X_{k} \rightarrow X_{k-1}$, and in general, $s_{r}$ is an index such that $m\left(F_{s_{r}}, 0\right)=m\left(f_{[k]}, 0\right)$, that is, $F_{s_{r}}$ has the smallest vanishing order among all components $F_{s}\left(s_{r}\right.$ may be vertical or not, and the choice of $\left\{s_{1}, \ldots, s_{r}\right\}$ need not be unique).

By definition, there is a canonical injection $\mathcal{O}_{X_{k}}(-1) \hookrightarrow \pi_{k}^{*} V_{k-1}$, and a composition with the projection $\left(\pi_{k-1}\right)_{*}$ (analogue for order $k-1$ of the arrow $\left(\pi_{k}\right)_{*}$ in sequence (6.4)) yields for any $k \geqslant 2$ a natural line bundle morphism

$$
\mathcal{O}_{X_{k}}(-1) \longleftrightarrow \pi_{k}^{*} V_{k-1} \stackrel{\left(\pi_{k}\right)^{*}\left(\pi_{k-1}\right)^{*}}{\longrightarrow} \pi_{k}^{*} \mathcal{O}_{X_{k-1}}(-1),
$$

which admits precisely $D_{k}=P\left(T_{X_{k-1} / X_{k-2}}\right) \subset P\left(V_{k-1}\right)=X_{k}$ as its zero divisor (clearly, $D_{k}$ is a hyperplane subbundle of $X_{k}$ ). Hence we find

$$
\mathcal{O}_{X_{k}}(1)=\pi_{k}^{*} \mathcal{O}_{X_{k-1}}(1) \otimes \mathcal{O}\left(D_{k}\right) .
$$

Now, we consider the composition of projections

$$
\pi_{j, k}=\pi_{j+1} \circ \cdots \circ \pi_{k-1} \circ \pi_{k}: X_{k} \longrightarrow X_{j} .
$$

Then $\pi_{0, k}: X_{k} \rightarrow X_{0}=X$ is a locally trivial holomorphic fiber bundle over $X$, and the fibers $X_{k, x}=\pi_{0, k}^{-1}(x)$ are $k$-stage towers of $\mathbb{P}^{r-1}$-bundles. Since we have (in both directions) morphisms $\left(\mathbb{C}^{r}, T_{\mathbb{C}^{r}}\right) \leftrightarrow(X, V)$ of directed manifolds which are bijective on the level of bundle morphisms, the fibers are all isomorphic to a "universal" non-singular projective algebraic variety of dimension $k(r-1)$ which we will denote by $\mathcal{R}_{r, k}$; it is not hard to see that $\mathcal{R}_{r, k}$ is rational (as will indeed follow from the proof of Theorem 7.11 below).

\section{B. Semple tower of Singular DiREcted VARIETIES}

Let $(X, V)$ be a directed variety. We assume $X$ non-singular, but here $V$ is allowed to have singularities. We are going to give a natural definition of the $\operatorname{Semple} \operatorname{tower}\left(X_{k}, V_{k}\right)$ in that case.

Let us take $X^{\prime}=X \backslash \operatorname{Sing}(V)$ and $V^{\prime}=V_{\uparrow X^{\prime}}$. By subsection 6.A, we have a well defined Semple tower $\left(X_{k}^{\prime}, V_{k}^{\prime}\right)$ over the Zariski open set $X^{\prime}$. We also have an "absolute" Semple tower $\left(X_{k}^{a}, V_{k}^{a}\right)$ obtained from $\left(X_{0}^{a}, V_{0}^{a}\right)=\left(X, T_{X}\right)$, which is non-singular. The injection $V^{\prime} \subset T_{X}$ induces by functoriality (cf. (5.5)) an injection

$$
\left(X_{k}^{\prime}, V_{k}^{\prime}\right) \subset\left(X_{k}^{a}, V_{k}^{a}\right)
$$


6.12. Definition. Let $(X, V)$ be a directed variety, with $X$ non-singular. When $\operatorname{Sing}(V) \neq \emptyset$, we define $X_{k}$ and $V_{k}$ to be the respective closures of $X_{k}^{\prime}, V_{k}^{\prime}$ associated with $X^{\prime}=X \backslash \operatorname{Sing}(V)$ and $V^{\prime}=V_{\uparrow X^{\prime}}$, where the closure is taken in the non-singular absolute Semple tower $\left(X_{k}^{a}, V_{k}^{a}\right)$ obtained from $\left(X_{0}^{a}, V_{0}^{a}\right)=\left(X, T_{X}\right)$.

We leave the reader check that the following functoriality property still holds.

6.13. Fonctoriality. If $\Phi:(X, V) \rightarrow(Y, W)$ is a morphism of directed varieties such that $\Phi_{*}$ : $T_{X} \rightarrow \Phi^{*} T_{Y}$ is injective (i.e. $\Phi$ is an immersion), then there is a corresponding natural morphism $\Phi_{[k]}:\left(X_{k}, V_{k}\right) \rightarrow\left(Y_{k}, W_{k}\right)$ at the level of Semple bundles. If one merely assumes that the differential $\Phi_{*}: V \rightarrow \Phi^{*} W$ is non-zero, there is still a natural meromorphic map $\Phi_{[k]}:\left(X_{k}, V_{k}\right) \rightarrow\left(Y_{k}, W_{k}\right)$ for all $k \geqslant 0$.

In case $V$ is singular, the $k$-th stage $X_{k}$ of the Semple tower will also be singular, but we can replace $\left(X_{k}, V_{k}\right)$ by a suitable modification $\left(\widehat{X}_{k}, \widehat{V}_{k}\right)$ if we want to work with a non-singular model $\widehat{X}_{k}$ of $X_{k}$. The exceptional set of $\widehat{X}_{k}$ over $X_{k}$ can be chosen to lie above $\operatorname{Sing}(V) \subset X$, and proceeding inductively with respect to $k$, we can also arrange the modifications in such a way that we get a tower structure $\left(\widehat{X}_{k+1}, \widehat{V}_{k+1}\right) \rightarrow\left(\widehat{X}_{k}, \widehat{V}_{k}\right)$; however, in general, it will not be possible to achieve that $\widehat{V}_{k}$ is a subbundle of $T_{\widehat{X}_{k}}$.

\section{Jet DifFERENTIALS}

\section{A. Green-Griffiths Jet Differentials}

We first introduce the concept of jet differentials in the sense of Green-Griffiths [GrGr80]. The goal is to provide an intrinsic geometric description of holomorphic differential equations that a germ of curve $f:(\mathbb{C}, 0) \rightarrow X$ may satisfy. In the sequel, we fix a directed manifold $(X, V)$ and suppose implicitly that all germs of curves $f$ are tangent to $V$.

Let $\mathbb{G}_{k}$ be the group of germs of $k$-jets of biholomorphisms of $(\mathbb{C}, 0)$, that is, the group of germs of biholomorphic maps

$$
t \mapsto \varphi(t)=a_{1} t+a_{2} t^{2}+\cdots+a_{k} t^{k}, \quad a_{1} \in \mathbb{C}^{*}, a_{j} \in \mathbb{C}, j \geqslant 2,
$$

in which the composition law is taken modulo terms $t^{j}$ of degree $j>k$. Then $\mathbb{G}_{k}$ is a $k$-dimensional nilpotent complex Lie group, which admits a natural fiberwise right action on $J_{k} V$. The action consists of reparametrizing $k$-jets of maps $f:(\mathbb{C}, 0) \rightarrow X$ by a biholomorphic change of parameter $\varphi:(\mathbb{C}, 0) \rightarrow(\mathbb{C}, 0)$, that is, $(f, \varphi) \mapsto f \circ \varphi$. There is an exact sequence of groups

$$
1 \rightarrow \mathbb{G}_{k}^{\prime} \rightarrow \mathbb{G}_{k} \rightarrow \mathbb{C}^{*} \rightarrow 1
$$

where $\mathbb{G}_{k} \rightarrow \mathbb{C}^{*}$ is the obvious morphism $\varphi \mapsto \varphi^{\prime}(0)$, and $\mathbb{G}_{k}^{\prime}=\left[\mathbb{G}_{k}, \mathbb{G}_{k}\right]$ is the group of $k$-jets of biholomorphisms tangent to the identity. Moreover, the subgroup $\mathbb{H} \simeq \mathbb{C}^{*}$ of homotheties $\varphi(t)=\lambda t$ is a (non-normal) subgroup of $\mathbb{G}_{k}$, and we have a semi-direct decomposition $\mathbb{G}_{k}=\mathbb{G}_{k}^{\prime} \ltimes \mathbb{H}$. The corresponding action on $k$-jets is described in coordinates by

$$
\lambda \cdot\left(f^{\prime}, f^{\prime \prime}, \ldots, f^{(k)}\right)=\left(\lambda f^{\prime}, \lambda^{2} f^{\prime \prime}, \ldots, \lambda^{k} f^{(k)}\right) .
$$

Following [GrGr80], we introduce the vector bundle $E_{k, m}^{\mathrm{GG}} V^{*} \rightarrow X$ whose fibers are complex valued polynomials $Q\left(f^{\prime}, f^{\prime \prime}, \ldots, f^{(k)}\right)$ on the fibers of $J_{k} V$, of weighted degree $m$ with respect to the $\mathbb{C}^{*}$ action defined by $\mathbb{H}$, that is, such that

$$
Q\left(\lambda f^{\prime}, \lambda^{2} f^{\prime \prime}, \ldots, \lambda^{k} f^{(k)}\right)=\lambda^{m} Q\left(f^{\prime}, f^{\prime \prime}, \ldots, f^{(k)}\right)
$$

for all $\lambda \in \mathbb{C}^{*}$ and $\left(f^{\prime}, f^{\prime \prime}, \ldots, f^{(k)}\right) \in J_{k} V$. Here we view $\left(f^{\prime}, f^{\prime \prime}, \ldots, f^{(k)}\right)$ as indeterminates with components

$$
\left(\left(f_{1}^{\prime}, \ldots, f_{r}^{\prime}\right) ;\left(f_{1}^{\prime \prime}, \ldots, f_{r}^{\prime \prime}\right) ; \ldots ;\left(f_{1}^{(k)}, \ldots, f_{r}^{(k)}\right)\right) \in\left(\mathbb{C}^{r}\right)^{k}
$$


Notice that the concept of polynomial on the fibers of $J_{k} V$ makes sense, for all coordinate changes $z \mapsto w=\Psi(z)$ on $X$ induce polynomial transition automorphisms on the fibers of $J_{k} V$, given by a formula

$$
(\Psi \circ f)^{(j)}=\Psi^{\prime}(f) \cdot f^{(j)}+\sum_{s=2}^{s=j} \sum_{j_{1}+j_{2}+\cdots+j_{s}=j} c_{j_{1} \ldots j_{s}} \Psi^{(s)}(f) \cdot\left(f^{\left(j_{1}\right)}, \ldots, f^{\left(j_{s}\right)}\right)
$$

with suitable integer constants $c_{j_{1} \ldots j_{s}}$ (this is easily checked by induction on $s$ ). In the "absolute case" $V=T_{X}$, we simply write $E_{k, m}^{\mathrm{GG}} T_{X}^{*}=E_{k, m}^{\mathrm{GG}}$. If $V \subset V^{\prime} \subset V^{a}:=T_{X}$ are holomorphic subbundles, there are natural inclusions

$$
J_{k} V \subset J_{k} V^{\prime} \subset J_{k} V^{a}, \quad X_{k} \subset X_{k}^{\prime} \subset X_{k}^{a} .
$$

The restriction morphisms induce surjective arrows

$$
E_{k, m}^{\mathrm{GG}} T_{X}^{*} \rightarrow E_{k, m}^{\mathrm{GG}} V^{*} \rightarrow E_{k, m}^{\mathrm{GG}} V^{*},
$$

and in particular $E_{k, m}^{\mathrm{GG}} V^{*}$ can be seen as a quotient of $E_{k, m}^{\mathrm{GG}} T_{X}^{*}$. (The notation $V^{*}$ is used here to make the contravariance property implicit from the notation). Another useful consequence of these inclusions is that one can extend the definition of $J_{k} V$ and $X_{k}$ to the case where $V$ is an arbitrary linear space, simply by taking the closure of $J_{k} V_{X \backslash \operatorname{Sing}(V)}$ and $X_{k \mid X \backslash \operatorname{Sing}(V)}$ in the smooth bundles $J_{k} X$ and $X_{k}^{a}$, respectively.

If $Q \in E_{k, m}^{\mathrm{GG}} V^{*}$ is decomposed into multihomogeneous components of multidegree $\left(\ell_{1}, \ell_{2}, \ldots, \ell_{k}\right)$ in $f^{\prime}, f^{\prime \prime}, \ldots, f^{(k)}$ (the decomposition is of course coordinate dependent), these multidegrees must satisfy the relation

$$
\ell_{1}+2 \ell_{2}+\cdots+k \ell_{k}=m \text {. }
$$

The bundle $E_{k, m}^{\mathrm{GG}} V^{*}$ will be called the bundle of jet differentials of order $k$ and weighted degree $m$. It is clear from (7.2) that a coordinate change $f \mapsto \Psi \circ f$ transforms every monomial $\left(f^{(\bullet)}\right)^{\ell}=$ $\left(f^{\prime}\right)^{\ell_{1}}\left(f^{\prime \prime}\right)^{\ell_{2}} \cdots\left(f^{(k)}\right)^{\ell_{k}}$ of partial weighted degree $|\ell|_{s}:=\ell_{1}+2 \ell_{2}+\cdots+s \ell_{s}, 1 \leqslant s \leqslant k$, into a polynomial $\left((\Psi \circ f)^{(\bullet)}\right)^{\ell}$ in $\left(f^{\prime}, f^{\prime \prime}, \ldots, f^{(k)}\right)$ whose non-zero monomials have the same partial weighted degree of order $s$ if $\ell_{s+1}=\cdots=\ell_{k}=0$, and a larger or equal partial degree of order $s$ otherwise. Hence, for each $s=1, \ldots, k$, we get a well defined (i.e., coordinate invariant) decreasing filtration $F_{s}^{\bullet}$ on $E_{k, m}^{\mathrm{GG}} V^{*}$ as follows:

$$
F_{s}^{p}\left(E_{k, m}^{\mathrm{GG}} V^{*}\right)=\left\{\begin{array}{l}
Q\left(f^{\prime}, f^{\prime \prime}, \ldots, f^{(k)}\right) \in E_{k, m}^{\mathrm{GG}} V^{*} \text { involving } \\
\text { only monomials }\left(f^{(\bullet)}\right)^{\ell} \text { with }|\ell|_{s} \geqslant p
\end{array}\right\}, \quad \forall p \in \mathbb{N} .
$$

The graded terms $\operatorname{Gr}_{k-1}^{p}\left(E_{k, m}^{\mathrm{GG}} V^{*}\right)$ associated with the filtration $F_{k-1}^{p}\left(E_{k, m}^{\mathrm{GG}} V^{*}\right)$ are precisely the homogeneous polynomials $Q\left(f^{\prime}, \ldots, f^{(k)}\right)$ whose monomials $\left(f^{\bullet}\right)^{\ell}$ all have partial weighted degree $|\ell|_{k-1}=p$ (hence their degree $\ell_{k}$ in $f^{(k)}$ is such that $m-p=k \ell_{k}$, and $\operatorname{Gr}_{k-1}^{p}\left(E_{k, m}^{\mathrm{GG}} V^{*}\right)=0$ unless $k \mid m-p)$. The transition automorphisms of the graded bundle are induced by coordinate changes $f \mapsto \Psi \circ f$, and they are described by substituting the arguments of $Q\left(f^{\prime}, \ldots, f^{(k)}\right)$ according to formula (7.2), namely $f^{(j)} \mapsto(\Psi \circ f)^{(j)}$ for $j<k$, and $f^{(k)} \mapsto \Psi^{\prime}(f) \circ f^{(k)}$ for $j=k$ (when $j=k$, the other terms fall in the next stage $F_{k-1}^{p+1}$ of the filtration). Therefore $f^{(k)}$ behaves as an element of $V \subset T_{X}$ under coordinate changes. We thus find

$$
G_{k-1}^{m-k \ell_{k}}\left(E_{k, m}^{\mathrm{GG}} V^{*}\right)=E_{k-1, m-k \ell_{k}}^{\mathrm{GG}} V^{*} \otimes S^{\ell_{k}} V^{*} .
$$

Combining all filtrations $F_{s}^{\bullet}$ together, we find inductively a filtration $F^{\bullet}$ on $E_{k, m}^{\mathrm{GG}} V^{*}$ such that the graded terms are

$$
\operatorname{Gr}^{\ell}\left(E_{k, m}^{\mathrm{GG}} V^{*}\right)=S^{\ell_{1}} V^{*} \otimes S^{\ell_{2}} V^{*} \otimes \cdots \otimes S^{\ell_{k}} V^{*}, \quad \ell \in \mathbb{N}^{k}, \quad|\ell|_{k}=m .
$$


The bundles $E_{k, m}^{\mathrm{GG}} V^{*}$ have other interesting properties. In fact,

$$
E_{k, \bullet}^{\mathrm{GG}} V^{*}:=\bigoplus_{m \geqslant 0} E_{k, m}^{\mathrm{GG}} V^{*}
$$

is in a natural way a bundle of graded algebras (the product is obtained simply by taking the product of polynomials). There are natural inclusions $E_{k, \bullet}^{\mathrm{GG}} V^{*} \subset E_{k+1, \bullet}^{\mathrm{GG}} V^{*}$ of algebras, and hence $E_{\infty, \bullet}^{\mathrm{GG}} V^{*}=\bigcup_{k \geqslant 0} E_{k, \bullet}^{\mathrm{GG}} V^{*}$ is also an algebra. Moreover, the sheaf of holomorphic sections $\mathcal{O}\left(E_{\infty, \bullet}^{\mathrm{GG}} V^{*}\right)$ admits a canonical derivation $D^{\mathrm{GG}}$ given by a collection of $\mathbb{C}$-linear maps

$$
D^{\mathrm{GG}}: \mathcal{O}\left(E_{k, m}^{\mathrm{GG}} V^{*}\right) \rightarrow \mathcal{O}\left(E_{k+1, m+1}^{\mathrm{GG}} V^{*}\right),
$$

constructed in the following way. A holomorphic section of $E_{k, m}^{\mathrm{GG}} V^{*}$ on a coordinate open set $\Omega \subset X$ can be seen as a differential operator on the space of germs $f:(\mathbb{C}, 0) \rightarrow \Omega$ of the form

$$
Q(f)=\sum_{\left|\alpha_{1}\right|+2\left|\alpha_{2}\right|+\cdots+k\left|\alpha_{k}\right|=m} a_{\alpha_{1} \ldots \alpha_{k}}(f)\left(f^{\prime}\right)^{\alpha_{1}}\left(f^{\prime \prime}\right)^{\alpha_{2}} \cdots\left(f^{(k)}\right)^{\alpha_{k}}
$$

in which the coefficients $a_{\alpha_{1} \ldots \alpha_{k}}$ are holomorphic functions on $\Omega$. Then $D^{\mathrm{GG}} Q$ is given by the formal derivative $\left(D^{\mathrm{GG}} Q\right)(f)(t)=d(Q(f)) / d t$ with respect to the 1-dimensional parameter $t$ in $f(t)$. For example, in dimension 2 , if $Q \in H^{0}\left(\Omega, \mathcal{O}\left(E_{2,4}^{\mathrm{GG}}\right)\right)$ is the section of weighted degree 4

$$
Q(f)=a\left(f_{1}, f_{2}\right) f_{1}^{\prime 3} f_{2}^{\prime}+b\left(f_{1}, f_{2}\right) f_{1}^{\prime \prime 2},
$$

we find that $D^{\mathrm{GG}} Q \in H^{0}\left(\Omega, \mathcal{O}\left(E_{3,5}^{\mathrm{GG}}\right)\right)$ is given by

$$
\begin{aligned}
\left(D^{\mathrm{GG}} Q\right)(f) & =\frac{\partial a}{\partial z_{1}}\left(f_{1}, f_{2}\right) f_{1}^{\prime 4} f_{2}^{\prime}+\frac{\partial a}{\partial z_{2}}\left(f_{1}, f_{2}\right) f_{1}^{\prime 3} f_{2}^{\prime 2}+\frac{\partial b}{\partial z_{1}}\left(f_{1}, f_{2}\right) f_{1}^{\prime} f_{1}^{\prime \prime 2} \\
& +\frac{\partial b}{\partial z_{2}}\left(f_{1}, f_{2}\right) f_{2}^{\prime} f_{1}^{\prime \prime 2}+a\left(f_{1}, f_{2}\right)\left(3 f_{1}^{\prime 2} f_{1}^{\prime \prime} f_{2}^{\prime}+f_{1}^{\prime 3} f_{2}^{\prime \prime}\right)+b\left(f_{1}, f_{2}\right) 2 f_{1}^{\prime \prime} f_{1}^{\prime \prime \prime} .
\end{aligned}
$$

Associated with the graded algebra bundle $E_{k, \bullet}^{\mathrm{GG}} V^{*}$, we have an analytic fiber bundle

$$
X_{k}^{\mathrm{GG}}:=\operatorname{Proj}\left(E_{k, \bullet}^{\mathrm{GG}} V^{*}\right)=\left(J_{k} V \backslash\{0\}\right) / \mathbb{C}^{*}
$$

over $X$, which has weighted projective spaces $\mathbb{P}\left(1^{[r]}, 2^{[r]}, \ldots, k^{[r]}\right)$ as fibers (these weighted projective spaces are singular for $k>1$, but they only have quotient singularities, see [Dol81]; here $J_{k} V \backslash\{0\}$ is the set of non-constant jets of order $k$; we refer e.g. to Hartshorne's book [Har77] for a definition of the Proj functor). As such, it possesses a canonical sheaf $\mathcal{O}_{X_{k}^{\mathrm{GG}}}(1)$ such that $\mathcal{O}_{X_{k}^{\mathrm{GG}}}(m)$ is invertible when $m$ is a multiple of $\operatorname{lcm}(1,2, \ldots, k)$. Under the natural projection $\pi_{k}: X_{k}^{\mathrm{GG}} \rightarrow X$, the direct image $\left(\pi_{k}\right)_{*} \mathcal{O}_{X_{k}^{G G}}(m)$ coincides with polynomials

$$
P\left(z ; \xi_{1}, \ldots, \xi_{k}\right)=\sum_{\alpha_{\ell} \in \mathbb{N}^{r}, 1 \leqslant \ell \leqslant k} a_{\alpha_{1} \ldots \alpha_{k}}(z) \xi_{1}^{\alpha_{1}} \ldots \xi_{k}^{\alpha_{k}}
$$

of weighted degree $\left|\alpha_{1}\right|+2\left|\alpha_{2}\right|+\ldots+k\left|\alpha_{k}\right|=m$ on $J^{k} V$ with holomorphic coefficients; in other words, we obtain precisely the sheaf of sections of the bundle $E_{k, m}^{\mathrm{GG}} V^{*}$ of jet differentials of order $k$ and degree $m$.

7.9. Proposition. By construction, if $\pi_{k}: X_{k}^{\mathrm{GG}} \rightarrow X$ is the natural projection, we have the direct image formula

$$
\left(\pi_{k}\right)_{*} \mathcal{O}_{X_{k}^{\mathrm{GG}}}(m)=\mathcal{O}\left(E_{k, m}^{\mathrm{GG}} V^{*}\right)
$$

for all $k$ and $m$. 


\section{B. INVARIANT JET DIFFERENTIALS}

In the geometric context, we are not really interested in the bundles $\left(J_{k} V \backslash\{0\}\right) / \mathbb{C}^{*}$ themselves, but rather in their quotients $\left(J_{k} V \backslash\{0\}\right) / \mathbb{G}_{k}$ (would such nice complex space quotients exist!). We will see that the Semple bundle $X_{k}$ constructed in $\S 6$ plays the role of such a quotient. First we introduce a canonical subalgebra of the bundle algebra $E_{k, \bullet}^{\mathrm{GG}} V^{*}$.

7.10. Definition. We introduce a subbundle $E_{k, m} V^{*} \subset E_{k, m}^{\mathrm{GG}} V^{*}$, called the bundle of invariant jet differentials of order $k$ and degree $m$, defined as follows: $E_{k, m} V^{*}$ is the set of polynomial differential operators $Q\left(f^{\prime}, f^{\prime \prime}, \ldots, f^{(k)}\right)$ which are invariant under arbitrary changes of parametrization, i.e., for every $\varphi \in \mathbb{G}_{k}$

$$
Q\left((f \circ \varphi)^{\prime},(f \circ \varphi)^{\prime \prime}, \ldots,(f \circ \varphi)^{(k)}\right)=\varphi^{\prime}(0)^{m} Q\left(f^{\prime}, f^{\prime \prime}, \ldots, f^{(k)}\right) .
$$

Alternatively, $E_{k, m} V^{*}=\left(E_{k, m}^{\mathrm{GG}} V^{*}\right)^{\mathbb{G}_{k}^{\prime}}$ is the set of invariants of $E_{k, m}^{\mathrm{GG}} V^{*}$ under the action of $\mathbb{G}_{k}^{\prime}$. Clearly, $E_{\infty, \bullet} V^{*}=\bigcup_{k \geqslant 0} \bigoplus_{m \geqslant 0} E_{k, m} V^{*}$ is a subalgebra of $E_{k, m}^{\mathrm{GG}} V^{*}$ (observe however that this algebra is not invariant under the derivation $D^{\mathrm{GG}}$, since e.g. $f_{j}^{\prime \prime}=D^{\mathrm{GG}} f_{j}$ is not an invariant polynomial). In addition to this, there are natural induced filtrations $F_{s}^{p}\left(E_{k, m} V^{*}\right)=E_{k, m} V^{*} \cap$ $F_{s}^{p}\left(E_{k, m}^{\mathrm{GG}} V^{*}\right)$ (all locally trivial over $X$ ). These induced filtrations will play an important role later on.

7.11. Theorem. Suppose that $V$ has rank $r \geqslant 2$. Let $\pi_{0, k}: X_{k} \longrightarrow X$ be the Semple jet bundles constructed in section 6 , and let $J_{k} V^{\text {reg }}$ be the bundle of regular $k$-jets of maps $f:(\mathbb{C}, 0) \rightarrow X$, that $i s$, jets $f$ such that $f^{\prime}(0) \neq 0$.

(i) The quotient $J_{k} V^{\mathrm{reg}} / \mathbb{G}_{k}$ has the structure of a locally trivial bundle over $X$, and there is a holomorphic embedding $J_{k} V^{\mathrm{reg}} / \mathbb{G}_{k} \hookrightarrow X_{k}$ over $X$, which identifies $J_{k} V^{\mathrm{reg}} / \mathbb{G}_{k}$ with $X_{k}^{\mathrm{reg}}$ (thus $X_{k}$ is a relative compactification of $J_{k} V^{\mathrm{reg}} / \mathbb{G}_{k}$ over $\left.X\right)$.

(ii) The direct image sheaf

$$
\left(\pi_{0, k}\right)_{*} \mathcal{O}_{X_{k}}(m) \simeq \mathcal{O}\left(E_{k, m} V^{*}\right)
$$

can be identified with the sheaf of holomorphic sections of $E_{k, m} V^{*}$.

(iii) For every $m>0$, the relative base locus of the linear system $\left|\mathcal{O}_{X_{k}}(m)\right|$ is equal to the set $X_{k}^{\text {sing }}$ of singular $k$-jets. Moreover, $\mathcal{O}_{X_{k}}(1)$ is relatively big over $X$.

Proof. (i) For $f \in J_{k} V^{\text {reg }}$, the lifting $\tilde{f}$ is obtained by taking the derivative $\left(f,\left[f^{\prime}\right]\right)$ without any cancellation of zeroes in $f^{\prime}$, and hence we get a uniquely defined $(k-1)$-jet $f:(\mathbb{C}, 0) \rightarrow \widetilde{X}$. Inductively, we get a well defined $(k-j)$-jet $f_{[j]}$ in $X_{j}$, and the value $f_{[k]}(0)$ is independent of the choice of the representative $f$ for the $k$-jet. As the lifting process commutes with reparametrization, i.e., $(f \circ \varphi)^{\sim}=\widetilde{f} \circ \varphi$ and more generally $(f \circ \varphi)_{[k]}=f_{[k]} \circ \varphi$, we conclude that there is a well defined set-theoretic map

$$
J_{k} V^{\mathrm{reg}} / \mathbb{G}_{k} \rightarrow X_{k}^{\mathrm{reg}}, \quad f \bmod \mathbb{G}_{k} \mapsto f_{[k]}(0) .
$$

This map is better understood in coordinates as follows. Fix coordinates $\left(z_{1}, \ldots, z_{n}\right)$ near a point $x_{0} \in X$, such that $V_{x_{0}}=\operatorname{Vect}\left(\partial / \partial z_{1}, \ldots, \partial / \partial z_{r}\right)$. Let $f=\left(f_{1}, \ldots, f_{n}\right)$ be a regular $k$-jet tangent to $V$. Then there exists $i \in\{1,2, \ldots, r\}$ such that $f_{i}^{\prime}(0) \neq 0$, and there is a unique reparametrization $t=\varphi(\tau)$ such that $f \circ \varphi=g=\left(g_{1}, g_{2}, \ldots, g_{n}\right)$ with $g_{i}(\tau)=\tau$ (we just express the curve as a graph over the $z_{i}$-axis, by means of a change of parameter $\tau=f_{i}(t)$, i.e. $\left.t=\varphi(\tau)=f_{i}^{-1}(\tau)\right)$. Suppose $i=r$ for the simplicity of notation. The space $X_{k}$ is a $k$-stage tower of $\mathbb{P}^{r-1}$-bundles. In the corresponding inhomogeneous coordinates on these $\mathbb{P}^{r-1}$ 's, the point $f_{[k]}(0)$ is given by the collection of derivatives

$$
\left(\left(g_{1}^{\prime}(0), \ldots, g_{r-1}^{\prime}(0)\right) ;\left(g_{1}^{\prime \prime}(0), \ldots, g_{r-1}^{\prime \prime}(0)\right) ; \ldots ;\left(g_{1}^{(k)}(0), \ldots, g_{r-1}^{(k)}(0)\right)\right) .
$$

[Recall that the other components $\left(g_{r+1}, \ldots, g_{n}\right)$ can be recovered from $\left(g_{1}, \ldots, g_{r}\right)$ by integrating the differential system (5.10)]. Thus the map $J_{k} V^{\text {reg }} / \mathbb{G}_{k} \rightarrow X_{k}$ is a bijection onto $X_{k}^{\mathrm{reg}}$, and the fibers of these isomorphic bundles can be seen as unions of $r$ affine charts $\simeq\left(\mathbb{C}^{r-1}\right)^{k}$, associated with 
each choice of the axis $z_{i}$ used to describe the curve as a graph. The change of parameter formula $\frac{d}{d \tau}=\frac{1}{f_{r}^{\prime}(t)} \frac{d}{d t}$ expresses all derivatives $g_{i}^{(j)}(\tau)=d^{j} g_{i} / d \tau^{j}$ in terms of the derivatives $f_{i}^{(j)}(t)=d^{j} f_{i} / d t^{j}$

$$
\begin{aligned}
\left(g_{1}^{\prime}, \ldots, g_{r-1}^{\prime}\right) & =\left(\frac{f_{1}^{\prime}}{f_{r}^{\prime}}, \ldots, \frac{f_{r-1}^{\prime}}{f_{r}^{\prime}}\right) ; \\
\left(g_{1}^{\prime \prime}, \ldots, g_{r-1}^{\prime \prime}\right) & =\left(\frac{f_{1}^{\prime \prime} f_{r}^{\prime}-f_{r}^{\prime \prime} f_{1}^{\prime}}{f_{r}^{\prime 3}}, \ldots, \frac{f_{r-1}^{\prime \prime} f_{r}^{\prime}-f_{r}^{\prime \prime} f_{r-1}^{\prime}}{f_{r}^{\prime 3}}\right) ; \ldots ; \\
\left(g_{1}^{(k)}, \ldots, g_{r-1}^{(k)}\right) & =\left(\frac{f_{1}^{(k)} f_{r}^{\prime}-f_{r}^{(k)} f_{1}^{\prime}}{f_{r}^{\prime k+1}}, \ldots, \frac{f_{r-1}^{(k)} f_{r}^{\prime}-f_{r}^{(k)} f_{r-1}^{\prime}}{f_{r}^{\prime k+1}}\right)+(\text { order }<k) .
\end{aligned}
$$

Also, it is easy to check that $f_{r}^{\prime 2 k-1} g_{i}^{(k)}$ is an invariant polynomial in $f^{\prime}, f^{\prime \prime}, \ldots, f^{(k)}$ of total degree $2 k-1$, i.e., a section of $E_{k, 2 k-1}$.

(ii) Since the bundles $X_{k}$ and $E_{k, m} V^{*}$ are both locally trivial over $X$, it is sufficient to identify sections $\sigma$ of $\mathcal{O}_{X_{k}}(m)$ over a fiber $X_{k, x}=\pi_{0, k}^{-1}(x)$ with the fiber $E_{k, m} V_{x}^{*}$, at any point $x \in X$. Let $f \in J_{k} V_{x}^{\text {reg }}$ be a regular $k$-jet at $x$. By (6.6), the derivative $f_{[k-1]}^{\prime}(0)$ defines an element of the fiber of $\mathcal{O}_{X_{k}}(-1)$ at $f_{[k]}(0) \in X_{k}$. Hence we get a well defined complex valued operator

$$
Q\left(f^{\prime}, f^{\prime \prime}, \ldots, f^{(k)}\right)=\sigma\left(f_{[k]}(0)\right) \cdot\left(f_{[k-1]}^{\prime}(0)\right)^{m} .
$$

Clearly, $Q$ is holomorphic on $J_{k} V_{x}^{\text {reg }}$ (by the holomorphicity of $\sigma$ ), and the $\mathbb{G}_{k}$-invariance condition of Definition 7.10 is satisfied since $f_{[k]}(0)$ does not depend on reparametrization and

$$
(f \circ \varphi)_{[k-1]}^{\prime}(0)=f_{[k-1]}^{\prime}(0) \varphi^{\prime}(0) .
$$

Now, $J_{k} V_{x}^{\text {reg }}$ is the complement of a linear subspace of codimension $r$ in $J_{k} V_{x}$, and hence $Q$ extends holomorphically to all of $J_{k} V_{x} \simeq\left(\mathbb{C}^{r}\right)^{k}$ by Riemann's extension theorem (here we use the hypothesis $r \geqslant 2$; if $r=1$, the situation is anyway not interesting since $X_{k}=X$ for all $k$ ). Thus $Q$ admits an everywhere convergent power series

$$
Q\left(f^{\prime}, f^{\prime \prime}, \ldots, f^{(k)}\right)=\sum_{\alpha_{1}, \alpha_{2}, \ldots, \alpha_{k} \in \mathbb{N}^{r}} a_{\alpha_{1} \ldots \alpha_{k}}\left(f^{\prime}\right)^{\alpha_{1}}\left(f^{\prime \prime}\right)^{\alpha_{2}} \cdots\left(f^{(k)}\right)^{\alpha_{k}} .
$$

The $\mathbb{G}_{k}$-invariance asserted in Definition 7.10 implies in particular that $Q$ must be multihomogeneous in the sense of (7.1), and thus $Q$ must be a polynomial. We conclude that $Q \in E_{k, m} V_{x}^{*}$, as desired.

Conversely, for all $w$ in a neighborhood of any given point $w_{0} \in X_{k, x}$, we can find a holomorphic family of germs $f_{w}:(\mathbb{C}, 0) \rightarrow X$ such that $\left(f_{w}\right)_{[k]}(0)=w$ and $\left(f_{w}\right)_{[k-1]}^{\prime}(0) \neq 0$ (just take the projections to $X$ of integral curves of $\left(X_{k}, V_{k}\right)$ integrating a nonvanishing local holomorphic section of $V_{k}$ near $\left.w_{0}\right)$. Then every $Q \in E_{k, m} V_{x}^{*}$ yields a holomorphic section $\sigma$ of $\mathcal{O}_{X_{k}}(m)$ over the fiber $X_{k, x}$ by putting

$$
\sigma(w)=Q\left(f_{w}^{\prime}, f_{w}^{\prime \prime}, \ldots, f_{w}^{(k)}\right)(0)\left(\left(f_{w}\right)_{[k-1]}^{\prime}(0)\right)^{-m}
$$

(iii) By what we saw in (i)-(ii), every section $\sigma$ of $\mathcal{O}_{X_{k}}(m)$ over the fiber $X_{k, x}$ is given by a polynomial $Q \in E_{k, m} V_{x}^{*}$, and this polynomial can be expressed on the Zariski open chart $f_{r}^{\prime} \neq 0$ of $X_{k, x}^{\text {reg }}$ as

$$
Q\left(f^{\prime}, f^{\prime \prime}, \ldots, f^{(k)}\right)=f_{r}^{\prime m} \widehat{Q}\left(g^{\prime}, g^{\prime \prime}, \ldots, g^{(k)}\right),
$$

where $\widehat{Q}$ is a polynomial and $g$ is the reparametrization of $f$ such that $g_{r}(\tau)=\tau$. In fact $\widehat{Q}$ is obtained from $Q$ by substituting $f_{r}^{\prime}=1$ and $f_{r}^{(j)}=0$ for $j \geqslant 2$, and conversely $Q$ can be recovered easily from $\widehat{Q}$ by using the substitutions (7.12).

In this context, the jet differentials $f \mapsto f_{1}^{\prime}, \ldots, f \mapsto f_{r}^{\prime}$ can be viewed as sections of $\mathcal{O}_{X_{k}}(1)$ on a neighborhood of the fiber $X_{k, x}$. Since these sections vanish exactly on $X_{k}^{\text {sing }}$, the relative base locus of $\mathcal{O}_{X_{k}}(m)$ is contained in $X_{k}^{\text {sing }}$ for every $m>0$. We see that $\mathcal{O}_{X_{k}}(1)$ is big by considering the 
sections of $\mathcal{O}_{X_{k}}(2 k-1)$ associated with the polynomials $Q\left(f^{\prime}, \ldots, f^{(k)}\right)=f_{r}^{\prime 2 k-1} g_{i}^{(j)}, 1 \leqslant i \leqslant r-1$, $1 \leqslant j \leqslant k$; indeed, these sections separate all points in the open chart $f_{r}^{\prime} \neq 0$ of $X_{k, x}^{\mathrm{reg}}$.

Now, we check that every section $\sigma$ of $\mathcal{O}_{X_{k}}(m)$ over $X_{k, x}$ must vanish on $X_{k, x}^{\text {sing }}$. Pick an arbitrary element $w \in X_{k}^{\text {sing }}$ and a germ of curve $f:(\mathbb{C}, 0) \rightarrow X$ such that $f_{[k]}(0)=w, f_{[k-1]}^{\prime}(0) \neq 0$ and $s=m(f, 0) \gg 0$ (such an $f$ exists by [Dem95, Corollary 5.14]). There are local coordinates $\left(z_{1}, \ldots, z_{n}\right)$ on $X$ such that $f(t)=\left(f_{1}(t), \ldots, f_{n}(t)\right)$ where $f_{r}(t)=t^{s}$. Let $Q, \widehat{Q}$ be the polynomials associated with $\sigma$ in these coordinates and let $\left(f^{\prime}\right)^{\alpha_{1}}\left(f^{\prime \prime}\right)^{\alpha_{2}} \cdots\left(f^{(k)}\right)^{\alpha_{k}}$ be a monomial occurring in $Q$, with $\alpha_{j} \in \mathbb{N}^{r},\left|\alpha_{j}\right|=\ell_{j}, \ell_{1}+2 \ell_{2}+\cdots+k \ell_{k}=m$. Putting $\tau=t^{s}$, the curve $t \mapsto f(t)$ becomes a Puiseux expansion $\tau \mapsto g(\tau)=\left(g_{1}(\tau), \ldots, g_{r-1}(\tau), \tau\right)$ in which $g_{i}$ is a power series in $\tau^{1 / s}$, starting with exponents of $\tau$ at least equal to 1 . The derivative $g^{(j)}(\tau)$ may involve negative powers of $\tau$, but the exponent is always $\geqslant 1+\frac{1}{s}-j$. Hence the Puiseux expansion of $\widehat{Q}\left(g^{\prime}, g^{\prime \prime}, \ldots, g^{(k)}\right)$ can only involve powers of $\tau$ of exponent $\geqslant-\max _{\ell}\left(\left(1-\frac{1}{s}\right) \ell_{2}+\cdots+\left(k-1-\frac{1}{s}\right) \ell_{k}\right)$. Finally $f_{r}^{\prime}(t)=$ $s t^{s-1}=s \tau^{1-1 / s}$, and so the lowest exponent of $\tau$ in $Q\left(f^{\prime}, \ldots, f^{(k)}\right)$ is at least equal to

$$
\begin{aligned}
\left(1-\frac{1}{s}\right) m-\max _{\ell} & \left(\left(1-\frac{1}{s}\right) \ell_{2}+\cdots+\left(k-1-\frac{1}{s}\right) \ell_{k}\right) \\
& \geqslant \min _{\ell}\left(1-\frac{1}{s}\right) \ell_{1}+\left(1-\frac{1}{s}\right) \ell_{2}+\cdots+\left(1-\frac{k-1}{s}\right) \ell_{k},
\end{aligned}
$$

where the minimum is taken over all monomials $\left(f^{\prime}\right)^{\alpha_{1}}\left(f^{\prime \prime}\right)^{\alpha_{2}} \cdots\left(f^{(k)}\right)^{\alpha_{k}},\left|\alpha_{j}\right|=\ell_{j}$, occurring in $Q$. Choosing $s \geqslant k$, we already find that the minimal exponent is positive, and hence $Q\left(f^{\prime}, \ldots, f^{(k)}\right)(0)=0$, so that $\sigma(w)=0$ by $(7.14)$.

Theorem 7.11 (iii) shows that $\mathcal{O}_{X_{k}}(1)$ is never relatively ample over $X$ for $k \geqslant 2$. In order to overcome this difficulty, we define for every $a_{\bullet}=\left(a_{1}, \ldots, a_{k}\right) \in \mathbb{Z}^{k}$ a line bundle $\mathcal{O}_{X_{k}}\left(a_{\bullet}\right)$ on $X_{k}$ such that

$$
\mathcal{O}_{X_{k}}\left(a_{\bullet}\right)=\pi_{1, k}^{*} \mathcal{O}_{X_{1}}\left(a_{1}\right) \otimes \pi_{2, k}^{*} \mathcal{O}_{X_{2}}\left(a_{2}\right) \otimes \cdots \otimes \mathcal{O}_{X_{k}}\left(a_{k}\right) .
$$

By (6.9), we have $\pi_{j, k}^{*} \mathcal{O}_{X_{j}}(1)=\mathcal{O}_{X_{k}}(1) \otimes \mathcal{O}_{X_{k}}\left(-\pi_{j+1, k}^{*} D_{j+1}-\cdots-D_{k}\right)$. Therefore by putting $D_{j}^{*}=\pi_{j+1, k}^{*} D_{j+1}$ for $1 \leqslant j \leqslant k-1$ and $D_{k}^{*}=0$, we find an identity

$$
\begin{aligned}
& \mathcal{O}_{X_{k}}\left(a_{\bullet}\right)=\mathcal{O}_{X_{k}}\left(b_{k}\right) \otimes \mathcal{O}_{X_{k}}\left(-b_{\bullet} \cdot D^{*}\right), \quad \text { where } \\
& b_{\bullet}=\left(b_{1}, \ldots, b_{k}\right) \in \mathbb{Z}^{k}, \quad b_{j}=a_{1}+\cdots+a_{j}, \\
& b_{\bullet} \cdot D^{*}=\sum_{1 \leqslant j \leqslant k-1} b_{j} \pi_{j+1, k}^{*} D_{j+1} .
\end{aligned}
$$

In particular, if $b_{\bullet} \in \mathbb{N}^{k}$, i.e., $a_{1}+\cdots+a_{j} \geqslant 0$, we get a morphism

$$
\mathcal{O}_{X_{k}}\left(a_{\bullet}\right)=\mathcal{O}_{X_{k}}\left(b_{k}\right) \otimes \mathcal{O}_{X_{k}}\left(-b_{\bullet} \cdot D^{*}\right) \rightarrow \mathcal{O}_{X_{k}}\left(b_{k}\right) .
$$

The following result gives a sufficient condition for the relative nefness or ampleness of weighted jet bundles. Let us recall that a line bundle $L \rightarrow X$ on a projective variety $X$ is said to be nef if $L \cdot C \geqslant 0$ for all irreducible algebraic curves $C \subset X$, and that a vector bundle $E \rightarrow X$ is said to be nef if $\mathcal{O}_{\mathbb{P}(E)}(1)$ is nef on $\mathbb{P}(E):=P\left(E^{*}\right)$; any vector bundle generated by global sections is nef (cf. [DePS94] for more details).

7.19. Proposition. Take a very ample line bundle $A$ on $X$, and consider on $X_{k}$ the line bundle

$$
L_{k}=\mathcal{O}_{X_{k}}\left(3^{k-1}, 3^{k-2}, \ldots, 3,1\right) \otimes \pi_{k, 0}^{*} A^{\otimes 3^{k}}
$$

defined inductively by $L_{0}=A$ and $L_{k}=\mathcal{O}_{X_{k}}(1) \otimes \pi_{k, k-1}^{*} L_{k-1}^{\otimes 3}$. Then $V_{k}^{*} \otimes L_{k}^{\otimes 2}$ is a nef vector bundle on $X_{k}$, which is in fact generated by its global sections, for all $k \geqslant 0$. Equivalently, for all $k \geqslant 1$,

$$
L_{k}^{\prime}=\mathcal{O}_{X_{k}}(1) \otimes \pi_{k, k-1}^{*} L_{k-1}^{\otimes 2}=\mathcal{O}_{X_{k}}\left(2 \cdot 3^{k-2}, 2 \cdot 3^{k-3}, \ldots, 6,2,1\right) \otimes \pi_{k, 0}^{*} A^{\otimes 2 \cdot 3^{k-1}}
$$


is nef over $X_{k}$ and generated by sections.

The statement concerning $L_{k}^{\prime}$ is obtained by projectivizing the vector bundle $E=V_{k-1}^{*} \otimes L_{k-1}^{\otimes 2}$ on $X_{k-1}$, whose associated tautological line bundle is $\mathcal{O}_{\mathbb{P}(E)}(1)=L_{k}^{\prime}$ on $\mathbb{P}(E)=P\left(V_{k-1}\right)=X_{k}$. Also one gets inductively that

$$
L_{k}=\mathcal{O}_{\mathbb{P}\left(V_{k-1} \otimes L_{k-1}^{\otimes 2}\right)}(1) \otimes \pi_{k, k-1}^{*} L_{k-1} \quad \text { is very ample on } X_{k} .
$$

Proof. Let $X \subset \mathbb{P}^{N}$ be the embedding provided by $A$, so that $A=\mathcal{O}_{\mathbb{P}^{N}}(1)_{\mid X}$. As is well known, if $Q$ is the tautological quotient vector bundle on $\mathbb{P}^{N}$, the twisted cotangent bundle

$$
T_{\mathbb{P}^{N}}^{*} \otimes \mathcal{O}_{\mathbb{P}^{N}}(2)=\Lambda^{N-1} Q
$$

is nef; hence its quotients $T_{X}^{*} \otimes A^{\otimes 2}$ and $V_{0}^{*} \otimes L_{0}^{\otimes 2}=V^{*} \otimes A^{\otimes 2}$ are nef (any tensor power of nef vector bundles is nef, and so is any quotient). We now proceed by induction, assuming $V_{k-1}^{*} \otimes L_{k-1}^{\otimes 2}$ to be nef, $k \geqslant 1$. By taking the second wedge power of the central term in $\left(6.4^{\prime}\right)$, we get an injection

$$
0 \longrightarrow T_{X_{k} / X_{k-1}} \longrightarrow \Lambda^{2}\left(\pi_{k}^{\star} V_{k-1} \otimes \mathcal{O}_{X_{k}}(1)\right) \text {. }
$$

By dualizing and twisting with $\mathcal{O}_{X_{k-1}}(2) \otimes \pi_{k}^{\star} L_{k-1}^{\otimes 2}$, we find a surjection

$$
\pi_{k}^{\star} \Lambda^{2}\left(V_{k-1}^{\star} \otimes L_{k-1}\right) \longrightarrow T_{X_{k} / X_{k-1}}^{\star} \otimes \mathcal{O}_{X_{k}}(2) \otimes \pi_{k}^{\star} L_{k-1}^{\otimes 2} \longrightarrow 0 .
$$

By the induction hypothesis, we see that $T_{X_{k} / X_{k-1}}^{\star} \otimes \mathcal{O}_{X_{k}}(2) \otimes \pi_{k}^{\star} L_{k-1}^{\otimes 2}$ is nef. Next, the dual of (6.4) yields an exact sequence

$$
0 \longrightarrow \mathcal{O}_{X_{k}}(1) \longrightarrow V_{k}^{\star} \longrightarrow T_{X_{k} / X_{k-1}}^{\star} \longrightarrow 0 .
$$

As an extension of nef vector bundles is nef, the nefness of $V_{k}^{*} \otimes L_{k}^{\otimes 2}$ will follow if we check that $\mathcal{O}_{X_{k}}(1) \otimes L_{k}^{\otimes 2}$ and $T_{X_{k} / X_{k-1}}^{\star} \otimes L_{k}^{\otimes 2}$ are both nef. However, this follows again from the induction hypothesis if we observe that the latter implies

$$
L_{k} \geqslant \pi_{k, k-1}^{*} L_{k-1} \quad \text { and } \quad L_{k} \geqslant \mathcal{O}_{X_{k}}(1) \otimes \pi_{k, k-1}^{*} L_{k-1},
$$

in the sense that $L^{\prime \prime} \geqslant L^{\prime}$ if the "difference" $L^{\prime \prime} \otimes\left(L^{\prime}\right)^{-1}$ is nef. All statements remain valid if we replace "nef" with "generated by sections" in the above arguments.

7.21. Corollary. $A \mathbb{Q}$-line bundle $\mathcal{O}_{X_{k}}\left(a_{\bullet}\right) \otimes \pi_{k, 0}^{*} A^{\otimes p}$ with $a_{\bullet} \in \mathbb{Q}^{k}, p \in \mathbb{Q}$, is nef, resp. ample, on $X_{k}$ as soon as

$$
a_{j} \geqslant 3 a_{j+1} \text { for } j=1,2, \ldots, k-2 \text { and } a_{k-1} \geqslant 2 a_{k} \geqslant 0, p \geqslant 2 \sum a_{j},
$$

resp.

$$
a_{j} \geqslant 3 a_{j+1} \text { for } j=1,2, \ldots, k-2 \text { and } a_{k-1}>2 a_{k}>0, p>2 \sum a_{j} .
$$

Proof. This follows easily by taking convex combinations of the $L_{j}$ and $L_{j}^{\prime}$ and applying Proposition 7.19 and our observation (7.20).

7.22. Remark. As in Green-Griffiths [GrGr80], Riemann's extension theorem shows that for every meromorphic map $\Phi: X \rightarrow Y$ there are well-defined pull-back morphisms

$$
\Phi^{*}: H^{0}\left(Y, E_{k, m}^{\mathrm{GG}} T_{Y}^{*}\right) \rightarrow H^{0}\left(X, E_{k, m}^{\mathrm{GG}} T_{X}^{*}\right), \quad \Phi^{*}: H^{0}\left(Y, E_{k, m} T_{Y}^{*}\right) \rightarrow H^{0}\left(X, E_{k, m} T_{X}^{*}\right) .
$$

In particular the dimensions $h^{0}\left(X, E_{k, m}^{\mathrm{GG}} T_{X}^{*}\right)$ and $h^{0}\left(X, E_{k, m} T_{X}^{*}\right)$ are bimeromorphic invariants of $X$.

7.23. Remark. As $\mathbb{G}_{k}$ is a non-reductive group, it is not a priori clear that the graded ring $\mathcal{A}_{n, k, r}=\bigoplus_{m \in \mathbb{Z}} E_{k, m} V^{\star}$ (even pointwise over $X$ ) is finitely generated. This can be checked by hand ([Dem07a], [Dem07b]) for $n=2$ and $k \leqslant 4$. Rousseau [Rou06] also checked the case $n=3, k=3$, and then Merker [Mer08, Mer10] proved the finiteness for $n=2,3,4, k \leqslant 4$ and $n=2, k=5$. Recently, Bérczi and Kirwan [BeKi12] made an attempt to prove the finiteness in full generality, but it appears that the general case is still unsettled. 


\section{C. Semple tower of a Directed VARIETy of General type}

Even if $(X, V)$ is of general type, it is not true that $\left(X_{k}, V_{k}\right)$ is of general type: the fibers of $X_{k} \rightarrow X$ are towers of $\mathbb{P}^{r-1}$ bundles, and the canonical bundles of projective spaces are always negative! However, a twisted version holds true.

7.24. Lemma. If $(X, V)$ is of general type, there is a modification $(\widehat{X}, \widehat{V})$ such that all pairs $\left(\widehat{X}_{k}, \widehat{V}_{k}\right)$ of the associated Semple tower have a twisted canonical bundle $K_{\widehat{V}_{k}} \otimes \mathcal{O}_{\widehat{X}_{k}}(p)$ that is big when one multiplies $K_{\widehat{V}_{k}}$ by a suitable $\mathbb{Q}$-line bundle $\mathcal{O}_{\widehat{X}_{k}}(p), p \in \mathbb{Q}_{+}$.

Proof. First assume that $V$ has no singularities. The exact sequences (6.4) and $\left(6.4^{\prime}\right)$ provide

$$
K_{V_{k}}:=\operatorname{det} V_{k}^{*}=\operatorname{det}\left(T_{X_{k} / X_{k-1}}^{*}\right) \otimes \mathcal{O}_{X_{k}}(1)=\pi_{k, k-1}^{*} K_{V_{k-1}} \otimes \mathcal{O}_{X_{k}}(-(r-1)),
$$

where $r=\operatorname{rank}(V)$. Inductively we get

$$
K_{V_{k}}=\pi_{k, 0}^{*} K_{V} \otimes \mathcal{O}_{X_{k}}\left(-(r-1) 1_{\bullet}\right), \quad 1_{\bullet}=(1, \ldots, 1) \in \mathbb{N}^{k} .
$$

We know by [Dem95] that $\mathcal{O}_{X_{k}}\left(c_{\boldsymbol{\bullet}}\right)$ is relatively ample over $X$ when we take the special weight $c_{\bullet}=\left(23^{k-2}, \ldots, 23^{k-j-1}, \ldots, 6,2,1\right)$; hence

$$
K_{V_{k}} \otimes \mathcal{O}_{X_{k}}\left((r-1) 1_{\bullet}+\varepsilon c_{\bullet}\right)=\pi_{k, 0}^{*} K_{V} \otimes \mathcal{O}_{X_{k}}\left(\varepsilon c_{\bullet}\right)
$$

is big over $X_{k}$ for any sufficiently small positive rational number $\varepsilon \in \mathbb{Q}_{+}^{*}$. Thanks to Formula (1.9), we can in fact replace the weight $(r-1) 1_{\bullet}+\varepsilon c_{\bullet}$ by its total degree $p=(r-1) k+\varepsilon\left|c_{\bullet}\right| \in \mathbb{Q}_{+}$. The general case of a singular linear space follows by considering suitable "sufficiently high" modifications $\widehat{X}$ of $X$, the related directed structure $\widehat{V}$ on $\widehat{X}$, and embedding $\left(\widehat{X}_{k}, \widehat{V}_{k}\right)$ in the absolute Semple tower $\left(\widehat{X}_{k}^{a}, \widehat{V}_{k}^{a}\right)$ of $\widehat{X}$. We still have a well defined morphism of rank 1 sheaves

$$
\pi_{k, 0}^{*} K_{\widehat{V}} \otimes \mathcal{O}_{\widehat{X}_{k}}\left(-(r-1) 1_{\bullet}\right) \rightarrow K_{\widehat{V}_{k}}
$$

because the multiplier ideal sheaves involved at each stage behave according to the monotonicity principle applied to the projections $\pi_{k, k-1}^{a}: \widehat{X}_{k}^{a} \rightarrow \widehat{X}_{k-1}^{a}$ and their differentials $\left(\pi_{k, k-1}^{a}\right)_{*}$, which yield well-defined transposed morphisms from the $(k-1)$-st stage to the $k$-th stage at the level of exterior differential forms. Our contention follows.

\section{D. InduCED DiRECTED STRUCTURe on A SUbVARIETy of A JET BUndLe}

We discuss here the concept of induced directed structure for subvarieties of the Semple tower of a directed variety $(X, V)$. This will be very important to proceed inductively with the base loci of jet differentials. Let $Z$ be an irreducible algebraic subset of some $k$-jet bundle $X_{k}$ over $X, k \geqslant 0$. We define the linear subspace $W \subset T_{Z} \subset T_{X_{k} \mid Z}$ to be the closure

$$
W:=\overline{T_{Z^{\prime}} \cap V_{k}}
$$

taken on a suitable Zariski open set $Z^{\prime} \subset Z_{\text {reg }}$ where the intersection $T_{Z^{\prime}} \cap V_{k}$ has a constant rank and is a subbundle of $T_{Z^{\prime}}$. Alternatively, we could also take $W$ to be the closure of $T_{Z^{\prime}} \cap V_{k}$ in the $k$-th stage $\left(X_{k}^{a}, V_{k}^{a}\right)$ of the absolute Semple tower, which has the advantage of being non-singular. We say that $(Z, W)$ is the induced directed variety structure; this concept of induced structure already applies of course in the case $k=0$. If $f:\left(\mathbb{C}, T_{\mathbb{C}}\right) \rightarrow(X, V)$ satisfies $f_{[k]}(\mathbb{C}) \subset Z$, then

$$
\text { either } f_{[k]}(\mathbb{C}) \subset Z_{\alpha} \quad \text { or } \quad f_{[k]}^{\prime}(\mathbb{C}) \subset W,
$$

where $Z_{\alpha}$ is one of the connected components of $Z \backslash Z^{\prime}$ and $Z^{\prime}$ is chosen as in (7.27); especially, if $W=0$, we conclude that $f_{[k]}(\mathbb{C})$ must be contained in one of the $Z_{\alpha}$ 's. In the sequel, we always consider such a subvariety $Z$ of $X_{k}$ as a directed pair $(Z, W)$ by taking the induced structure described above. By (7.28), if we proceed by induction on $\operatorname{dim} Z$, the study of curves tangent to $V$ that have an order $k$ lifting $f_{[k]}(\mathbb{C}) \subset Z$ is reduced to the study of curves tangent to $(Z, W)$. Let us first quote the following easy observation. 
7.29. Observation. For $k \geqslant 1$, let $Z \subsetneq X_{k}$ be an irreducible algebraic subset that projects onto $X_{k-1}$, i.e. $\pi_{k, k-1}(Z)=X_{k-1}$. Then the induced directed variety $(Z, W) \subset\left(X_{k}, V_{k}\right)$, satisfies

$$
1 \leqslant \operatorname{rank} W<r:=\operatorname{rank}\left(V_{k}\right) .
$$

Proof. Take a Zariski open subset $Z^{\prime} \subset Z_{\text {reg }}$ such that $W^{\prime}=T_{Z^{\prime}} \cap V_{k}$ is a vector bundle over $Z^{\prime}$. Since $X_{k} \rightarrow X_{k-1}$ is a $\mathbb{P}^{r-1}$-bundle, $Z$ has codimension at most $(r-1)$ in $X_{k}$. Therefore rank $W \geqslant$ $\operatorname{rank} V_{k}-(r-1) \geqslant 1$. On the other hand, if we had $\operatorname{rank} W=\operatorname{rank} V_{k}$ generically, then $T_{Z^{\prime}}$ would contain $V_{k \mid Z^{\prime}}$, in particular it would contain all vertical directions $T_{X_{k}} / X_{k-1} \subset V_{k}$ that are tangent to the fibers of $X_{k} \rightarrow X_{k-1}$. By taking the flow along vertical vector fields, we would conclude that $Z^{\prime}$ is a union of fibers of $X_{k} \rightarrow X_{k-1}$ up to an algebraic set of smaller dimension, but this is excluded since $Z$ projects onto $X_{k-1}$ and $Z \subsetneq X_{k}$.

We introduce the following definition that slightly differs from the one given in [Dem14] - it is actually more flexible and more general.

7.30. Definition. For $k \geqslant 1$, let $Z \subset X_{k}$ be an irreducible algebraic subset of $X_{k}$ and $(Z, W)$ the induced directed structure. We assume moreover that $Z \not \subset D_{k}=P\left(T_{X_{k-1} / X_{k-2}}\right)$ (and put $D_{1}=\emptyset$ in what follows to avoid to have to single out the case $k=1)$. In this situation we say that $(Z, W)$ is of general type modulo the Semple tower $X_{\bullet} \rightarrow X$ if either $W=0$, or rank $W \geqslant 1$ and there exists $\ell \geqslant 0$ and $p \in \mathbb{Q} \geqslant 0$ such that

$$
K_{\widehat{W}_{\ell}}^{\bullet} \otimes \mathcal{O}_{\widehat{Z}_{\ell}}(p)=K_{\widehat{W}_{\ell}}^{\bullet} \otimes \mathcal{O}_{\widehat{X}_{k+\ell}}(p)_{\uparrow \widehat{Z}_{\ell}} \text { is big over } \widehat{Z}_{\ell},
$$

possibly after replacing $\left(Z_{\ell}, W_{\ell}\right)$ by a suitable (non-singular) modification $\left(\widehat{Z}_{\ell}, \widehat{W}_{\ell}\right)$ obtained via an embedded resolution of singularities

$$
\mu_{\ell}:\left(\widehat{Z}_{\ell} \subset \widehat{X}_{k+\ell}\right) \rightarrow\left(Z_{\ell} \subset X_{k+\ell}\right) .
$$

Notice that by (7.26), Condition (7.31) is satisfied if we assume the existence of $p \geqslant 0$ such that

$$
\pi_{k+\ell}^{*} K_{\widehat{W}}^{\bullet} \otimes \mathcal{O}_{\widehat{X}_{k+\ell}}(p)_{\uparrow \widehat{Z}_{\ell}} \text { is big over } \widehat{Z}_{\ell} \subset \widehat{X}_{k+\ell} .
$$

In fact we infer $(7.31)$ with $\mathcal{O}_{\widehat{Z}_{\ell}}(p)$ replaced by $\mathcal{O}_{\widehat{Z}_{\ell}}\left((0, \ldots, 0, p)+\left(r_{W}-1\right) 1.\right) \subset \mathcal{O}_{\widehat{Z}_{\ell}}\left(p+\left(r_{W}-1\right) \ell\right)$. As a consequence, (7.31) is satisfied if $K_{\widehat{W}}^{\bullet}$ is big (i.e. $(Z, W)$ is of general type), or if $\mathcal{O}_{\widehat{Z}_{\ell}}(1)$ is big on some $\widehat{Z}_{\ell}, \ell \geqslant 1$, but (7.32) is weaker than these two bigness conditions, since we only require that some combination is big. Also, we have the following easy observation.

7.33. Proposition. Let $(X, V)$ be a projective directed variety. Assume that there exist $\ell \geqslant 1$ and a weight $a_{\bullet} \in \mathbb{Q}_{>0}^{\ell}$ such that $\mathcal{O}_{X_{\ell}}\left(a_{\bullet}\right)$ is ample over $X_{\ell}$. Then every induced directed variety $(Z, W) \subset\left(X_{k}, V_{k}\right)$ is if general type modulo $X \bullet \rightarrow X$ for every $k \geqslant 1$.

Proof. Corollary 7.21 shows that for $\ell^{\prime}>\ell$ and a suitable weight $b_{\bullet} \in \mathbb{Q}^{\ell^{\prime}}$, the line bundle $\mathcal{O}_{X_{\ell^{\prime}}}\left(b_{\bullet}\right)$ is relatively ample with respect to the projection $X_{\ell^{\prime}} \rightarrow X_{\ell}$. From this, one deduces that the assumption also holds for arbitrary $\ell^{\prime}>\ell$ and a suitable weight $a_{\bullet}^{\prime} \in \mathbb{Q}_{>0}^{\ell^{\prime}}$. Now, we use (7.32), in combination with Lemma 2.9 (b); in fact, $\mathcal{O}_{\widehat{X}_{k+\ell}}(1)_{\uparrow \widehat{Z}_{\ell}}$ is big over $\widehat{Z}_{\ell} \subset \widehat{X}_{k+\ell}$ for $\ell \gg 1$, since we get many sections by pulling back the sections of $\mathcal{O}_{\widehat{X}_{\ell^{\prime}}}\left(m a_{\bullet}^{\prime}\right), \ell^{\prime}=k+\ell$, and by restricting them to $\widehat{Z}_{\ell}$.

\section{E. RELATION BETWEEN INVARIANT AND NON-INVARIANT JET DIFFERENTIALS}

We show here that the existence of $\mathbb{G}_{k}$-invariant global jet differentials is essentially equivalent to the existence of non-invariant ones. We have seen that the direct image sheaf

$$
\pi_{k, 0} \mathcal{O}_{X_{k}}(m):=E_{k, m} V^{*} \subset E_{k, m}^{\mathrm{GG}} V^{*}
$$


has a stalk at point $x \in X$ that consists of algebraic differential operators $P\left(f_{[k]}\right)$ acting on germs of $k$-jets $f:(\mathbb{C}, 0) \rightarrow(X, x)$ tangent to $V$, satisfying the invariance property

$$
P\left((f \circ \varphi)_{[k]}\right)=\left(\varphi^{\prime}\right)^{m} P\left(f_{[k]}\right) \circ \varphi
$$

whenever $\varphi \in \mathbb{G}_{k}$ is in the group of $k$-jets of biholomorphisms $\varphi:(\mathbb{C}, 0) \rightarrow(\mathbb{C}, 0)$. The right action $J_{k} V \times \mathbb{G}_{k} \rightarrow J_{k} V,(f, \varphi) \mapsto f \circ \varphi$ induces a dual left action of $\mathbb{G}_{k}$ on $\bigoplus_{m^{\prime} \leqslant m} E_{k, m^{\prime}}^{\mathrm{GG}} V^{*}$ by

$$
\mathbb{G}_{k} \times \bigoplus_{m^{\prime} \leqslant m} E_{k, m^{\prime}}^{\mathrm{GG}} V_{x}^{*} \rightarrow \bigoplus_{m^{\prime} \leqslant m} E_{k, m^{\prime}}^{\mathrm{GG}} V_{x}^{*},(\varphi, P) \mapsto \varphi^{*} P, \quad\left(\varphi^{*} P\right)\left(f_{[k]}\right)=P\left((f \circ \varphi)_{[k]}\right),
$$

so that $\psi^{*}\left(\varphi^{*} P\right)=(\psi \circ \varphi)^{*} P$. Notice that for a global curve $f:\left(\mathbb{C}, T_{\mathbb{C}}\right) \rightarrow(X, V)$ and a global operator $P \in H^{0}\left(X, E_{k, m}^{\mathrm{GG}} V^{*} \otimes F\right)$ we have to modify a little bit the definition to consider germs of curves at points $t_{0} \in \mathbb{C}$ other than 0 . This leads to putting

$$
\varphi^{*} P\left(f_{[k]}\right)\left(t_{0}\right)=P\left(\left(f \circ \varphi_{t_{0}}\right)_{[k]}\right)(0) \quad \text { where } \varphi_{t_{0}}(t)=t_{0}+\varphi(t), t \in D(0, \varepsilon) .
$$

The $\mathbb{C}^{*}$-action on a homogeneous polynomial of degree $m$ is simply $h_{\lambda}^{*} P=\lambda^{m} P$ for a dilation $h_{\lambda}(t)=\lambda t, \lambda \in \mathbb{C}^{*}$, but since $\varphi \circ h_{\lambda} \neq h_{\lambda} \circ \varphi$ in general, $\varphi^{*} P$ is no longer homogeneous when $P$ is. However, by expanding the derivatives of $t \mapsto f(\varphi(t))$ at $t=0$, we find an expression

$$
\left(\varphi^{*} P\right)\left(f_{[k]}\right)=\sum_{\alpha \in \mathbb{N}^{k},|\alpha|_{w}=m} \varphi^{(\alpha)}(0) P_{\alpha}\left(f_{[k]}\right),
$$

where $\alpha=\left(\alpha_{1}, \ldots, \alpha_{k}\right) \in \mathbb{N}^{k}, \varphi^{(\alpha)}=\left(\varphi^{\prime}\right)^{\alpha_{1}}\left(\varphi^{\prime \prime}\right)^{\alpha_{2}} \ldots\left(\varphi^{(k)}\right)^{\alpha_{k}},|\alpha|_{w}=\alpha_{1}+2 \alpha_{2}+\ldots+k \alpha_{k}$ is the weighted degree and $P_{\alpha}$ is a homogeneous polynomial. Since any additional derivative taken on $\varphi^{\prime}$ means one less derivative left for $f$, it is easy to see that for $P$ homogeneous of degree $m$ we have

$$
m_{\alpha}:=\operatorname{deg}\left(P_{\alpha}\right)=m-\left(\alpha_{2}+2 \alpha_{3}+\ldots+(k-1) \alpha_{k}\right)=\alpha_{1}+\alpha_{2}+\ldots+\alpha_{k},
$$

in particular $\operatorname{deg}\left(P_{\alpha}\right)<m$ unless $\alpha=(m, 0, \ldots, 0)$, in which case $P_{\alpha}=P$. Let us fix a nonzero global section $P \in H^{0}\left(X, E_{k, m}^{\mathrm{GG}} V^{*} \otimes F\right)$ for some line bundle $F$ over $X$, and pick a non-zero component $P_{\alpha_{0}}$ of minimum degree $m_{\alpha_{0}}$ in the decomposition of $P$ (of course $m_{\alpha_{0}}=m$ if and only if $P$ is already invariant). We have by construction

$$
P_{\alpha_{0}} \in H^{0}\left(X, E_{k, m_{\alpha_{0}}}^{\mathrm{GG}} V^{*} \otimes F\right) .
$$

We claim that $P_{\alpha_{0}}$ is $\mathbb{G}_{k}$-invariant. Otherwise, there is for each $\alpha$ a decomposition

$$
\left(\psi^{*} P_{\alpha}\right)\left(f_{[k]}\right)=\sum_{\beta \in \mathbb{N}^{k},|\beta|_{w}=m_{\alpha}} \psi^{(\beta)}(0) P_{\alpha, \beta}\left(f_{[k]}\right),
$$

and the non-invariance of $P_{\alpha_{0}}$ would yield some non-zero term $P_{\alpha_{0}, \beta_{0}}$ of degree

$$
\operatorname{deg}\left(P_{\alpha_{0}, \beta_{0}}\right)<\operatorname{deg}\left(P_{\alpha_{0}}\right) \leqslant \operatorname{deg}(P)=m .
$$

By replacing $f$ with $f \circ \psi$ in (7.36) and plugging (7.37) into it, we would get an identity of the form

$$
(\psi \circ \varphi)^{*} P\left(f_{[k]}\right)=\sum_{\alpha \in \mathbb{N}^{k}}(\psi \circ \varphi)^{(\alpha)}(0) P_{\alpha}\left(f_{[k]}\right)=\sum_{\alpha, \beta \in \mathbb{N}^{k}} \varphi^{(\alpha)}(0) \psi^{(\beta)}(0) P_{\alpha, \beta}\left(f_{[k]}\right),
$$

but the term in the middle would have all components of degree $\geqslant m_{\alpha_{0}}$, while the term on the right possesses a component of degree $<m_{\alpha_{0}}$ for a sufficiently generic choice of $\varphi$ and $\psi$, contradiction. Therefore, we have shown the existence of a non-zero invariant section

$$
P_{\alpha_{0}} \in H^{0}\left(X, E_{k, m_{\alpha_{0}}} V^{*} \otimes F\right), \quad m_{\alpha_{0}} \leqslant m .
$$




\section{8. $k$-JET METRICS WiTH NEGATIVE CURVATURE}

The goal of this section is to show that hyperbolicity is closely related to the existence of $k$ jet metrics with suitable negativity properties of the curvature. The connection between these properties is in fact a simple consequence of the Ahlfors-Schwarz lemma. Such ideas have been already developed long ago by Grauert-Reckziegel [GRe65], Kobayashi [Kob75] for 1-jet metrics (i.e., Finsler metrics on $T_{X}$ ) and by Cowen-Griffiths [CoGr76], Green-Griffiths [GrGr80] and Grauert [Gra89] for higher order jet metrics.

\section{A. Definition of $k$-Jet Metrics}

Even in the standard case $V=T_{X}$, the definition given below differs from that of [GrGr80], in which the $k$-jet metrics are not supposed to be $\mathbb{G}_{k}^{\prime}$-invariant. We prefer to deal here with $\mathbb{G}_{k}^{\prime}$ invariant objects, because they reflect better the intrinsic geometry. Grauert [Gra89] actually deals with $\mathbb{G}_{k}^{\prime}$-invariant metrics, but he apparently does not take care of the way the quotient space $J_{k}^{\text {reg }} V / \mathbb{G}_{k}$ can be compactified; also, his metrics are always induced by the Poincaré metric, and it is not at all clear whether these metrics have the expected curvature properties (see Problem 8.14 below). In the present situation, it is important to allow also Hermitian metrics possessing some singularities ("singular Hermitian metrics" in the sense of [Dem90b]).

8.1. Definition. Let $L \rightarrow X$ be a holomorphic line bundle over a complex manifold $X$. We say that $h$ is a singular metric on $L$ if for any trivialization $L_{\uparrow U} \simeq U \times \mathbb{C}$ of $L$, the metric is given by $|\xi|_{h}^{2}=|\xi|^{2} e^{-\varphi}$ for some real valued weight function $\varphi \in L_{\mathrm{loc}}^{1}(U)$. The curvature current of $L$ is then defined to be the closed $(1,1)$-current $\Theta_{L, h}=\frac{i}{2 \pi} \partial \bar{\partial} \varphi$, computed in the sense of distributions. We say that $h$ admits a closed subset $\Sigma \subset X$ as its degeneration set if $\varphi$ is locally bounded on $X \backslash \Sigma$ and is unbounded on a neighborhood of any point of $\Sigma$.

An especially useful situation is the case when the curvature of $h$ is positive definite. By this, we mean that there exists a smooth positive definite Hermitian metric $\omega$ and a continuous positive function $\varepsilon$ on $X$ such that $\Theta_{L, h} \geqslant \varepsilon \omega$ in the sense of currents, and we write in this case $\Theta_{L, h} \gg 0$. We need the following basic fact (quite standard when $X$ is projective algebraic); however we want to avoid any algebraicity assumption here, so as to cover potential applications to non algebraic complex tori.

8.2. Proposition. Let $L$ be a holomorphic line bundle on a compact complex manifold $X$.

(i) $L$ admits a singular Hermitian metric $h$ with positive definite curvature current $\Theta_{L, h} \gg 0$ if and only if $L$ is big. Now, define $B_{m}$ to be the base locus of the linear system $\left|H^{0}\left(X, L^{\otimes m}\right)\right|$ and let

$$
\Phi_{m}: X \backslash B_{m} \rightarrow \mathbb{P}^{N}
$$

be the corresponding meromorphic map. Let $\Sigma_{m}$ be the closed analytic set equal to the union of $B_{m}$ and of the set of points $x \in X \backslash B_{m}$ such that the fiber $\Phi_{m}^{-1}\left(\Phi_{m}(x)\right)$ is positive dimensional.

(ii) If $\Sigma_{m} \neq X$ and $G$ is any line bundle, the base locus of $L^{\otimes k} \otimes G^{-1}$ is contained in $\Sigma_{m}$ for $k$ large. As a consequence, $L$ admits a singular Hermitian metric $h$ with degeneration set $\Sigma_{m}$ and with $\Theta_{L, h}$ positive definite on $X$.

(iii) Conversely, if $L$ admits a Hermitian metric $h$ with degeneration set $\Sigma$ and positive definite curvature current $\Theta_{L, h}$, there exists an integer $m>0$ such that the base locus $B_{m}$ is contained in $\Sigma$ and $\Phi_{m}: X \backslash \Sigma \rightarrow \mathbb{P}_{m}$ is an embedding.

Proof. (i) is proved e.g. in [Dem90b, 92], and (ii) and (iii) are well-known results in the basic theory of linear systems.

We now come to the main definitions. By (6.6), every regular $k$-jet $f \in J_{k} V$ gives rise to an element $f_{[k-1]}^{\prime}(0) \in \mathcal{O}_{X_{k}}(-1)$. Thus, measuring the "norm of $k$-jets" is the same as taking a Hermitian metric on $\mathcal{O}_{X_{k}}(-1)$. 
8.3. Definition. A smooth, (resp. continuous, resp. singular) $k$-jet metric on a complex directed manifold $(X, V)$ is a Hermitian metric $h_{k}$ on the line bundle $\mathcal{O}_{X_{k}}(-1)$ over $X_{k}$ (i.e. a Finsler metric on the vector bundle $V_{k-1}$ over $\left.X_{k-1}\right)$, such that the weight functions $\varphi$ representing the metric are smooth (resp. continuous, $L_{\text {loc }}^{1}$ ). We let $\Sigma_{h_{k}} \subset X_{k}$ be the singularity set of the metric, i.e., the closed subset of points in a neighborhood of which the weight $\varphi$ is not locally bounded.

We will always assume here that the weight function $\varphi$ is quasi psh. Recall that a function $\varphi$ is said to be quasi psh if $\varphi$ is locally the sum of a plurisubharmonic function and of a smooth function (so that in particular $\varphi \in L_{\mathrm{loc}}^{1}$ ). Then the curvature current

$$
\Theta_{h_{k}^{-1}}\left(\mathcal{O}_{X_{k}}(1)\right)=\frac{i}{2 \pi} \partial \bar{\partial} \varphi .
$$

is well defined as a current and is locally bounded from below by a negative $(1,1)$-form with constant coefficients.

8.4. Definition. Let $h_{k}$ be a $k$-jet metric on $(X, V)$. We say that $h_{k}$ has negative jet curvature (resp. negative total jet curvature) if $\Theta_{h_{k}}\left(\mathcal{O}_{X_{k}}(-1)\right)$ is negative definite along the subbundle $V_{k} \subset T_{X_{k}}$ (resp. on all of $T_{X_{k}}$ ), i.e., if there is $\varepsilon>0$ and a smooth Hermitian metric $\omega_{k}$ on $T_{X_{k}}$ such that

$$
\left.\left\langle\Theta_{h_{k}^{-1}}\left(\mathcal{O}_{X_{k}}(1)\right)\right\rangle(\xi) \geqslant \varepsilon|\xi|_{\omega_{k}}^{2}, \quad \forall \xi \in V_{k} \subset T_{X_{k}} \quad \text { (resp. } \quad \forall \xi \in T_{X_{k}}\right) .
$$

(If the metric $h_{k}$ is not smooth, we suppose that its weights $\varphi$ are quasi psh, and the curvature inequality is taken in the sense of distributions.)

It is important to observe that for $k \geqslant 2$ there cannot exist any smooth Hermitian metric $h_{k}$ on $\mathcal{O}_{X_{k}}(1)$ with positive definite curvature along $T_{X_{k} / X}$, since $\mathcal{O}_{X_{k}}(1)$ is not relatively ample over $X$. However, it is relatively big, and Prop. 8.2 (i) shows that $\mathcal{O}_{X_{k}}(-1)$ admits a singular Hermitian metric with negative total jet curvature (whatever the singularities of the metric are) if and only if $\mathcal{O}_{X_{k}}(1)$ is big over $X_{k}$. It is therefore crucial to allow singularities in the metrics in Def. 8.4.

\section{B. SpeCial CASE OF 1-Jet Metrics}

A 1-jet metric $h_{1}$ on $\mathcal{O}_{X_{1}}(-1)$ is the same as a Finsler metric $N=\sqrt{h_{1}}$ on $V \subset T_{X}$. Assume until the end of this paragraph that $h_{1}$ is smooth. By the well known Kodaira embedding theorem, the existence of a smooth metric $h_{1}$ such that $\Theta_{h_{1}^{-1}}\left(\mathcal{O}_{X_{1}}(1)\right)$ is positive on all of $T_{X_{1}}$ is equivalent to $\mathcal{O}_{X_{1}}(1)$ being ample, that is, $V^{*}$ ample.

8.5 Remark. In the absolute case $V=T_{X}$, there are only few examples of varieties $X$ such that $T_{X}^{*}$ is ample, mainly quotients of the ball $\mathbb{B}_{n} \subset \mathbb{C}^{n}$ by a discrete cocompact group of automorphisms.

The 1-jet negativity condition considered in Definition 8.4 is much weaker. For example, if the Hermitian metric $h_{1}$ comes from a (smooth) Hermitian metric $h$ on $V$, then formula (5.15) implies that $h_{1}$ has negative total jet curvature (i.e. $\Theta_{h_{1}^{-1}}\left(\mathcal{O}_{X_{1}}(1)\right)$ is positive) if and only if $\left\langle\Theta_{V, h}\right\rangle(\zeta \otimes v)<0$ for all $\zeta \in T_{X} \backslash\{0\}, v \in V \backslash\{0\}$, that is, if $(V, h)$ is negative in the sense of Griffiths. On the other hand, $V_{1} \subset T_{X_{1}}$ consists by definition of tangent vectors $\tau \in T_{X_{1},(x,[v])}$ whose horizontal projection $H_{\tau}$ is proportional to $v$. Thus $\Theta_{h_{1}}\left(\mathcal{O}_{X_{1}}(-1)\right)$ is negative definite on $V_{1}$ if and only if $\Theta_{V, h}$ satisfies the much weaker condition that the holomorphic sectional curvature $\left\langle\Theta_{V, h}\right\rangle(v \otimes v)$ is negative on every complex line.

\section{C. VANISHING THEOREM FOR INVARIANT JET DIFFERENTIALS}

We now come back to the general situation of jets of arbitrary order $k$. Our first observation is the fact that the $k$-jet negativity property of the curvature becomes actually weaker and weaker as $k$ increases.

8.6. Lemma. Let $(X, V)$ be a compact complex directed manifold. If $(X, V)$ has a $(k-1)$-jet metric $h_{k-1}$ with negative jet curvature, then there is a $k$-jet metric $h_{k}$ with negative jet curvature such that $\Sigma_{h_{k}} \subset \pi_{k}^{-1}\left(\Sigma_{h_{k-1}}\right) \cup D_{k}$. (The same holds true for negative total jet curvature). 
Proof. Let $\omega_{k-1}, \omega_{k}$ be given smooth Hermitian metrics on $T_{X_{k-1}}$ and $T_{X_{k}}$. The hypothesis implies

$$
\left\langle\Theta_{h_{k-1}^{-1}}\left(\mathcal{O}_{X_{k-1}}(1)\right)\right\rangle(\xi) \geqslant \varepsilon|\xi|_{\omega_{k-1}}^{2}, \quad \forall \xi \in V_{k-1}
$$

for some constant $\varepsilon>0$. On the other hand, as $\mathcal{O}_{X_{k}}\left(D_{k}\right)$ is relatively ample over $X_{k-1}\left(D_{k}\right.$ is a hyperplane section bundle), there exists a smooth metric $\widetilde{h}$ on $\mathcal{O}_{X_{k}}\left(D_{k}\right)$ such that

$$
\left\langle\Theta_{\widetilde{h}}\left(\mathcal{O}_{X_{k}}\left(D_{k}\right)\right)\right\rangle(\xi) \geqslant \delta|\xi|_{\omega_{k}}^{2}-C\left|\left(\pi_{k}\right)_{*} \xi\right|_{\omega_{k-1}}^{2}, \quad \forall \xi \in T_{X_{k}}
$$

for some constants $\delta, C>0$. Combining both inequalities (the second one being applied to $\xi \in V_{k}$ and the first one to $\left.\left(\pi_{k}\right)_{*} \xi \in V_{k-1}\right)$, we get

$$
\begin{aligned}
\left\langle\Theta _ { ( \pi _ { k } ^ { * } h _ { k - 1 } ) ^ { - p } \widetilde { h } } \left(\pi_{k}^{*} \mathcal{O}_{X_{k-1}}(p) \otimes\right.\right. & \left.\left.\mathcal{O}_{X_{k}}\left(D_{k}\right)\right)\right\rangle(\xi) \\
& \geqslant \delta|\xi|_{\omega_{k}}^{2}+(p \varepsilon-C)\left|\left(\pi_{k}\right)_{*} \xi\right|_{\omega_{k-1}}^{2}, \quad \forall \xi \in V_{k} .
\end{aligned}
$$

Hence, for $p$ large enough, $\left(\pi_{k}^{*} h_{k-1}\right)^{-p} \widetilde{h}$ has positive definite curvature along $V_{k}$. Now, by (6.9), there is a sheaf injection

$$
\mathcal{O}_{X_{k}}(-p)=\pi_{k}^{*} \mathcal{O}_{X_{k-1}}(-p) \otimes \mathcal{O}_{X_{k}}\left(-p D_{k}\right) \hookrightarrow\left(\pi_{k}^{*} \mathcal{O}_{X_{k-1}}(p) \otimes \mathcal{O}_{X_{k}}\left(D_{k}\right)\right)^{-1}
$$

obtained by twisting with $\mathcal{O}_{X_{k}}\left((p-1) D_{k}\right)$. Therefore $h_{k}:=\left(\left(\pi_{k}^{*} h_{k-1}\right)^{-p} \widetilde{h}\right)^{-1 / p}=\left(\pi_{k}^{*} h_{k-1}\right) \widetilde{h}^{-1 / p}$ induces a singular metric on $\mathcal{O}_{X_{k}}(1)$ in which an additional degeneration divisor $p^{-1}(p-1) D_{k}$ appears. Hence we get $\Sigma_{h_{k}}=\pi_{k}^{-1} \Sigma_{h_{k-1}} \cup D_{k}$ and

$$
\Theta_{h_{k}^{-1}}\left(\mathcal{O}_{X_{k}}(1)\right)=\frac{1}{p} \Theta_{\left(\pi_{k}^{*} h_{k-1}\right)^{-p} \widetilde{h}}+\frac{p-1}{p}\left[D_{k}\right]
$$

is positive definite along $V_{k}$. The same proof works in the case of negative total jet curvature.

One of the main motivations for the introduction of $k$-jets metrics is the following list of algebraic sufficient conditions.

8.7. Algebraic sufficient conditions. We suppose here that $X$ is projective algebraic, and we make one of the additional assumptions (i), (ii) or (iii) below.

(i) Assume that there exist integers $k, m>0$ and $b_{\bullet} \in \mathbb{N}^{k}$ such that the line bundle $L:=\mathcal{O}_{X_{k}}(m) \otimes$ $\mathcal{O}_{X_{k}}\left(-b_{\bullet} \cdot D^{*}\right)$ is ample over $X_{k}$. Then there is a smooth Hermitian metric $h_{L}$ on $L$ with positive definite curvature on $X_{k}$. By means of the morphism $\mu: \mathcal{O}_{X_{k}}(-m) \rightarrow L^{-1}$, we get an induced metric $h_{k}=\left(\mu^{*} h_{L}^{-1}\right)^{1 / m}$ on $\mathcal{O}_{X_{k}}(-1)$ which is degenerate on the support of the zero $\operatorname{divisor} \operatorname{div}(\mu)=b_{\bullet} \cdot D^{*}$. Hence $\Sigma_{h_{k}}=\operatorname{Supp}\left(b_{\bullet} \cdot D^{*}\right) \subset X_{k}^{\text {sing }}$ and

$$
\Theta_{h_{k}^{-1}}\left(\mathcal{O}_{X_{k}}(1)\right)=\frac{1}{m} \Theta_{h_{L}}(L)+\frac{1}{m}\left[b_{\bullet} \cdot D^{*}\right] \geqslant \frac{1}{m} \Theta_{h_{L}}(L)>0 .
$$

In particular $h_{k}$ has negative total jet curvature.

(ii) Assume more generally that there exist integers $k, m>0$ and an ample line bundle $A$ on $X$ such that $H^{0}\left(X_{k}, \mathcal{O}_{X_{k}}(m) \otimes \pi_{0, k}^{*} A^{-1}\right)$ has non-zero sections $\sigma_{1}, \ldots, \sigma_{N}$. Let $Z \subset X_{k}$ be the base locus of these sections; necessarily $Z \supset X_{k}^{\text {sing }}$ by 7.11 (iii). By taking a smooth metric $h_{A}$ with positive curvature on $A$, we get a singular metric $h_{k}^{\prime}$ on $\mathcal{O}_{X_{k}}(-1)$ such that

$$
h_{k}^{\prime}(\xi)=\left(\sum_{1 \leqslant j \leqslant N}\left|\sigma_{j}(w) \cdot \xi^{m}\right|_{h_{A}^{-1}}^{2}\right)^{1 / m}, \quad w \in X_{k}, \quad \xi \in \mathcal{O}_{X_{k}}(-1)_{w} .
$$

Then $\Sigma_{h_{k}^{\prime}}=Z$, and by computing $\frac{i}{2 \pi} \partial \bar{\partial} \log h_{k}^{\prime}(\xi)$ we obtain

$$
\Theta_{h_{k}^{\prime-1}}\left(\mathcal{O}_{X_{k}}(1)\right) \geqslant \frac{1}{m} \pi_{0, k}^{*} \Theta_{A}
$$


By (7.17) and an induction on $k$, there exists $b_{\bullet} \in \mathbb{Q}_{+}^{k}$ such that $\mathcal{O}_{X_{k}}(1) \otimes \mathcal{O}_{X_{k}}\left(-b_{\bullet} \cdot D^{*}\right)$ is relatively ample over $X$. Hence $L=\mathcal{O}_{X_{k}}(1) \otimes \mathcal{O}_{X_{k}}\left(-b_{\bullet} \cdot D^{*}\right) \otimes \pi_{0, k}^{*} A^{\otimes p}$ is ample on $X$ for $p \gg 0$. The arguments used in (i) show that there is a $k$-jet metric $h_{k}^{\prime \prime}$ on $\mathcal{O}_{X_{k}}(-1)$ with $\Sigma_{h_{k}^{\prime \prime}}=\operatorname{Supp}\left(b_{\bullet} \cdot D^{*}\right)=X_{k}^{\text {sing }}$ and

$$
\Theta_{h_{k}^{\prime \prime}-1}\left(\mathcal{O}_{X_{k}}(1)\right)=\Theta_{L}+\left[b_{\bullet} \cdot D^{*}\right]-p \pi_{0, k}^{*} \Theta_{A},
$$

where $\Theta_{L}$ is positive definite on $X_{k}$. The metric $h_{k}=\left(h_{k}^{\prime m p} h_{k}^{\prime \prime}\right)^{1 /(m p+1)}$ then satisfies $\Sigma_{h_{k}}=\Sigma_{h_{k}^{\prime}}=$ $Z$ and

$$
\Theta_{h_{k}^{-1}}\left(\mathcal{O}_{X_{k}}(1)\right) \geqslant \frac{1}{m p+1} \Theta_{L}>0 .
$$

(iii) If $E_{k, m} V^{*}$ is ample, there is an ample line bundle $A$ and a sufficiently high symmetric power such that $S^{p}\left(E_{k, m} V^{*}\right) \otimes A^{-1}$ is generated by sections. These sections can be viewed as sections of $\mathcal{O}_{X_{k}}(m p) \otimes \pi_{0, k}^{*} A^{-1}$ over $X_{k}$, and their base locus is exactly $Z=X_{k}^{\text {sing }}$ by 7.11 (iii). Hence the $k$-jet metric $h_{k}$ constructed in (ii) has negative total jet curvature and satisfies $\Sigma_{h_{k}}=X_{k}^{\text {sing }}$.

An important fact, first observed by [GRe65] for 1-jet metrics and by [GrGr80] in the higher order case, is that $k$-jet negativity implies hyperbolicity. In particular, the existence of enough global jet differentials implies hyperbolicity.

8.8. Theorem. Let $(X, V)$ be a compact complex directed manifold. If $(X, V)$ has a $k$-jet metric $h_{k}$ with negative jet curvature, then every entire curve $f: \mathbb{C} \rightarrow X$ tangent to $V$ is such that $f_{[k]}(\mathbb{C}) \subset \Sigma_{h_{k}}$. In particular, if $\Sigma_{h_{k}} \subset X_{k}^{\text {sing }}$, then $(X, V)$ is hyperbolic.

Proof. The main idea is to use the Ahlfors-Schwarz lemma, following the approach of [GrGr80]. However we will give here all necessary details because our setting is slightly different. Assume that there is a $k$-jet metric $h_{k}$ as in the hypotheses of Theorem 8.8. Let $\omega_{k}$ be a smooth Hermitian metric on $T_{X_{k}}$. By hypothesis, there exists $\varepsilon>0$ such that

$$
\left\langle\Theta_{h_{k}^{-1}}\left(\mathcal{O}_{X_{k}}(1)\right)\right\rangle(\xi) \geqslant \varepsilon|\xi|_{\omega_{k}}^{2} \quad \forall \xi \in V_{k} .
$$

Moreover, by (6.4), $\left(\pi_{k}\right)_{*}$ maps $V_{k}$ continuously to $\mathcal{O}_{X_{k}}(-1)$ and the weight $e^{\varphi}$ of $h_{k}$ is locally bounded from above. Hence there is a constant $C>0$ such that

$$
\left|\left(\pi_{k}\right)_{*} \xi\right|_{h_{k}}^{2} \leqslant C|\xi|_{\omega_{k}}^{2}, \quad \forall \xi \in V_{k} .
$$

Combining these inequalities, we find

$$
\left\langle\Theta_{h_{k}^{-1}}\left(\mathcal{O}_{X_{k}}(1)\right)\right\rangle(\xi) \geqslant \frac{\varepsilon}{C}\left|\left(\pi_{k}\right)_{*} \xi\right|_{h_{k}}^{2}, \quad \forall \xi \in V_{k} .
$$

Now, let $f: \Delta_{R} \rightarrow X$ be a non-constant holomorphic map tangent to $V$ on the disk $\Delta_{R}$. We use the line bundle morphism (6.6)

$$
F=f_{[k-1]}^{\prime}: T_{\Delta_{R}} \rightarrow f_{[k]}^{*} \mathcal{O}_{X_{k}}(-1)
$$

to obtain a pull-back metric

$$
\gamma=\gamma_{0}(t) d t \otimes d \bar{t}=F^{*} h_{k} \quad \text { on } T_{\Delta_{R}} .
$$

If $f_{[k]}\left(\Delta_{R}\right) \subset \Sigma_{h_{k}}$ then $\gamma \equiv 0$. Otherwise, $F(t)$ has isolated zeroes at all singular points of $f_{[k-1]}$ and so $\gamma(t)$ vanishes only at these points and at points of the degeneration set $\left(f_{[k]}\right)^{-1}\left(\Sigma_{h_{k}}\right)$ which is a polar set in $\Delta_{R}$. At other points, the Gaussian curvature of $\gamma$ satisfies

$$
\frac{i \partial \bar{\partial} \log \gamma_{0}(t)}{\gamma(t)}=\frac{-2 \pi\left(f_{[k]}\right)^{*} \Theta_{h_{k}}\left(\mathcal{O}_{X_{k}}(-1)\right)}{F^{*} h_{k}}=\frac{\left\langle\Theta_{h_{k}^{-1}}\left(\mathcal{O}_{X_{k}}(1)\right)\right\rangle\left(f_{[k]}^{\prime}(t)\right)}{\left|f_{[k-1]}^{\prime}(t)\right|_{h_{k}}^{2}} \geqslant \frac{\varepsilon}{C},
$$

since $f_{[k-1]}^{\prime}(t)=\left(\pi_{k}\right)_{*} f_{[k]}^{\prime}(t)$. The Ahlfors-Schwarz lemma 4.2 implies that $\gamma$ can be compared with the Poincaré metric as follows:

$$
\gamma(t) \leqslant \frac{2 C}{\varepsilon} \frac{R^{-2}|d t|^{2}}{\left(1-|t|^{2} / R^{2}\right)^{2}} \Longrightarrow\left|f_{[k-1]}^{\prime}(t)\right|_{h_{k}}^{2} \leqslant \frac{2 C}{\varepsilon} \frac{R^{-2}}{\left(1-|t|^{2} / R^{2}\right)^{2}} .
$$


If $f: \mathbb{C} \rightarrow X$ is an entire curve tangent to $V$ such that $f_{[k]}(\mathbb{C}) \not \subset \Sigma_{h_{k}}$, the above estimate implies as $R \rightarrow+\infty$ that $f_{[k-1]}$ must be a constant, and hence also $f$. Now, if $\Sigma_{h_{k}} \subset X_{k}^{\text {sing }}$, the inclusion $f_{[k]}(\mathbb{C}) \subset \Sigma_{h_{k}}$ implies $f^{\prime}(t)=0$ at every point. Therefore $f$ is a constant and $(X, V)$ is hyperbolic.

Combining Theorem 8.8 with 8.7 (ii) and (iii), we get the following consequences.

8.9. Vanishing theorem. Assume that there exist integers $k, m>0$ and an ample line bundle $L$ on $X$ such that $H^{0}\left(X_{k}, \mathcal{O}_{X_{k}}(m) \otimes \pi_{0, k}^{*} L^{-1}\right) \simeq H^{0}\left(X, E_{k, m} V^{*} \otimes L^{-1}\right)$ has non-zero sections $\sigma_{1}, \ldots, \sigma_{N}$. Let $Z \subset X_{k}$ be the base locus of these sections. Then every entire curve $f: \mathbb{C} \rightarrow X$ tangent to $V$ is such that $f_{[k]}(\mathbb{C}) \subset Z$. In other words, for every global $\mathbb{G}_{k}$-invariant polynomial differential operator $P$ with values in $L^{-1}$, every entire curve $f$ must satisfy the algebraic differential equation $P\left(f_{[k]}\right)=0$.

8.10. Corollary. Let $(X, V)$ be a compact complex directed manifold. If $E_{k, m} V^{*}$ is ample for some positive integers $k, m$, then $(X, V)$ is hyperbolic.

8.11. Remark. Green and Griffiths [GrGr80] stated that Theorem 8.9 is even true for sections $\sigma_{j} \in H^{0}\left(X, E_{k, m}^{\mathrm{GG}}\left(V^{*}\right) \otimes L^{-1}\right)$, in the special case $V=T_{X}$ they consider. This is proved below in $\S 8 . \mathrm{D}$; the reader is also referred to Siu and Yeung [SiYe97] for a proof based on a use of Nevanlinna theory and the logarithmic derivative lemma (the original proof given in [GrGr80] does not seem to be complete, as it relies on an unsettled pointwise version of the Ahlfors-Schwarz lemma for general jet differentials); other proofs seem to have been circulating in the literature in the last years. Let us first give a very short proof in the case where $f$ is supposed to have a bounded derivative (thanks to the Brody criterion, this is enough if one is merely interested in proving hyperbolicity; thus Corollary 8.10 will be valid with $E_{k, m}^{\mathrm{GG}} V^{*}$ in place of $\left.E_{k, m} V^{*}\right)$. In fact, if $f^{\prime}$ is bounded, one can apply the Cauchy inequalities to all components $f_{j}$ of $f$ with respect to a finite collection of coordinate patches covering $X$. As $f^{\prime}$ is bounded, we can do this on sufficiently small discs $D(t, \delta) \subset \mathbb{C}$ of constant radius $\delta>0$. Therefore all derivatives $f^{\prime}, f^{\prime \prime}, \ldots f^{(k)}$ are bounded. From this we conclude that $\sigma_{j}(f)$ is a bounded section of $f^{*} L^{-1}$. Its norm $\left|\sigma_{j}(f)\right|_{L^{-1}}$ (with respect to any positively curved metric ||$_{L}$ on $\left.L\right)$ is a bounded subharmonic function, which is moreover strictly subharmonic at all points where $f^{\prime} \neq 0$ and $\sigma_{j}(f) \neq 0$. This is a contradiction unless $f$ is constant or $\sigma_{j}(f) \equiv 0$.

The above results justify the following definition and problems.

8.12. Definition. We say that $X$, resp. $(X, V)$, has non degenerate negative $k$-jet curvature if there exists a $k$-jet metric $h_{k}$ on $\mathcal{O}_{X_{k}}(-1)$ with negative jet curvature such that $\Sigma_{h_{k}} \subset X_{k}^{\text {sing }}$.

8.13. Conjecture. Let $(X, V)$ be a compact directed manifold. Then $(X, V)$ is hyperbolic if and only if $(X, V)$ has nondegenerate negative $k$-jet curvature for $k$ large enough.

This is probably a hard problem. In fact, it is shown in [Dem97, Section 8] that the smallest admissible integer $k$ must depend on the geometry of $X$ and need not be uniformly bounded as soon as $\operatorname{dim} X \geqslant 2$ (even in the absolute case $V=T_{X}$ ). On the other hand, if $(X, V)$ is hyperbolic, we get for each integer $k \geqslant 1$ a generalized Kobayashi-Royden metric $\mathbf{k}_{\left(X_{k-1}, V_{k-1}\right)}$ on $V_{k-1}$ (see Definition 2.1), which can be also viewed as a $k$-jet metric $h_{k}$ on $\mathcal{O}_{X_{k}}(-1)$; we will call it the Grauert $k$-jet metric of $(X, V)$, although it formally differs from the jet metric considered in [Gra89] (see also [DGr91]). By looking at the projection $\pi_{k}:\left(X_{k}, V_{k}\right) \rightarrow\left(X_{k-1}, V_{k-1}\right)$, we see that the sequence $h_{k}$ is monotonic, namely $\pi_{k}^{*} h_{k} \leqslant h_{k+1}$ for every $k$. If $(X, V)$ is hyperbolic, then $h_{1}$ is nondegenerate and therefore by monotonicity $\Sigma_{h_{k}} \subset X_{k}^{\text {sing }}$ for $k \geqslant 1$. Conversely, if the Grauert metric satisfies $\Sigma_{h_{k}} \subset X_{k}^{\text {sing }}$, it is easy to see that $(X, V)$ is hyperbolic. The following problem is thus especially meaningful.

8.14. Problem. Estimate the $k$-jet curvature $\Theta_{h_{k}^{-1}}\left(\mathcal{O}_{X_{k}}(1)\right)$ of the Grauert metric $h_{k}$ on $\left(X_{k}, V_{k}\right)$ as $k$ tends to $+\infty$. 


\section{D. VANISHING THEOREM FOR NON-INVARIANT JET DIFFERENTIALS}

As an application of the arguments developed in $\S 7$.E, we indicate here a proof of the basic vanishing theorem for non-invariant jet differentials. This version has been first proved in full generality by Siu [Siu97] (cf. also [Dem97]), with a different and more involved technique based on Nevanlinna theory and the logarithmic derivative lemma.

8.15. Theorem. Let $(X, V)$ be a projective directed and $A$ an ample divisor on $X$. Then one has $P\left(f ; f^{\prime}, f^{\prime \prime}, \ldots, f^{(k)}\right)=0$ for every entire curve $f:\left(\mathbb{C}, T_{\mathbb{C}}\right) \rightarrow(X, V)$ and every global section $P \in H^{0}\left(X, E_{k, m}^{\mathrm{GG}} V^{*} \otimes \mathcal{O}(-A)\right)$.

Sketch of proof. In general, we know by Theorem 8.9 that the result is true when $P$ is invariant, i.e. for $P \in H^{0}\left(X, E_{k, m} V^{*} \otimes \mathcal{O}(-A)\right)$. Now, we prove Theorem 8.15 by induction on $k$ and $m$ (simultaneously for all directed varieties). Let $Z \subset X_{k}$ be the base locus of all polynomials $Q \in H^{0}\left(X, E_{k, m^{\prime}}^{\mathrm{GG}} V^{*} \otimes \mathcal{O}(-A)\right)$ with $m^{\prime}<m$. A priori, this defines merely an algebraic set in the Green-Griffiths bundle $X_{k}^{\mathrm{GG}}=\left(J_{k} V \backslash\{0\}\right) / \mathbb{C}^{*}$, but since the global polynomials $\varphi^{*} Q$ also enter the game, we know that the base locus is $\mathbb{G}_{k}$-invariant, and thus descends to $X_{k}$. Let $f:\left(\mathbb{C}, T_{\mathbb{C}}\right) \rightarrow(X, V)$. By the induction hypothesis, we know that $f_{[k]}(\mathbb{C}) \subset Z$. Therefore $f$ can also be viewed as a entire curve drawn in the directed variety $(Z, W)$ induced by $\left(X_{k}, V_{k}\right)$. By (7.36), we have a decomposition

$$
\left(\varphi^{*} P\right)\left(g_{[k]}\right)=\sum_{\alpha \in \mathbb{N}^{k},|\alpha|_{w}=m} \varphi^{(\alpha)}(0) P_{\alpha}\left(g_{[k]}\right), \quad \text { with } \operatorname{deg}\left(P_{\alpha}\right)<\operatorname{deg}(P) \text { for } \alpha \neq(m, 0, \ldots, 0),
$$

and since $P_{\alpha}\left(g_{[k]}\right)=0$ for all germs of curves $g$ of $(Z, W)$ when $\alpha \neq(m, 0, \ldots, 0)$, we conclude that $P$ defines an invariant jet differential when it is restricted to $(Z, W)$, in other words it still defines a section of

$$
H^{0}\left(Z,\left(\mathcal{O}_{X_{k}}(m) \otimes \pi_{k, 0}^{*} \mathcal{O}_{X}(-A)\right)_{\lceil Z}\right) .
$$

We can then apply the Ahlfors-Schwarz lemma in the way we did it in §8.C to conclude that $P\left(f_{[k]}\right)=0$.

\section{Morse inequalities and the Green-Griffiths-Lang Conjecture}

The goal of this section is to study the existence and properties of entire curves $f: \mathbb{C} \rightarrow X$ drawn in a complex irreducible $n$-dimensional variety $X$, and more specifically to show that they must satisfy certain global algebraic or differential equations as soon as $X$ is projective of general type. By means of holomorphic Morse inequalities and a probabilistic analysis of the cohomology of jet spaces, it is possible to prove a significant step of the generalized Green-Griffiths-Lang conjecture. The use of holomorphic Morse inequalities was first suggested in [Dem07a], and then carried out in an algebraic context by S. Diverio in his $\mathrm{PhD}$ work ([Div08, Div09]). The general more analytic and more powerful results presented here first appeared in [Dem11, Dem12].

\section{A. InTRODUCTION}

Let $(X, V)$ be a directed variety. By definition, proving the algebraic degeneracy of an entire curve $f ;\left(\mathbb{C}, T_{\mathbb{C}}\right) \rightarrow(X, V)$ means finding a non-zero polynomial $P$ on $X$ such that $P(f)=0$. As already explained in $\S 8$, all known methods of proof are based on establishing first the existence of certain algebraic differential equations $P\left(f ; f^{\prime}, f^{\prime \prime}, \ldots, f^{(k)}\right)=0$ of some order $k$, and then trying to find enough such equations so that they cut out a proper algebraic locus $Y \subsetneq X$. We use for this global sections of $H^{0}\left(X, E_{k, m}^{\mathrm{GG}} V^{*} \otimes \mathcal{O}(-A)\right)$ where $A$ is ample, and apply the fundamental vanishing theorem 8.15. It is expected that the global sections of $H^{0}\left(X, E_{k, m}^{\mathrm{GG}} V^{*} \otimes \mathcal{O}(-A)\right)$ are precisely those which ultimately define the algebraic locus $Y \subsetneq X$ where the curve $f$ should lie. The problem is then reduced to (i) showing that there are many non-zero sections of $H^{0}\left(X, E_{k, m}^{\mathrm{GG}} V^{*} \otimes \mathcal{O}(-A)\right)$ and (ii) understanding what is their joint base locus. The first part of this program is the main result of this section. 
9.1. Theorem. Let $(X, V)$ be a directed projective variety such that $K_{V}$ is big and let $A$ be an ample divisor. Then for $k \gg 1$ and $\delta \in \mathbb{Q}_{+}$small enough, $\delta \leqslant c(\log k) / k$, the number of sections $h^{0}\left(X, E_{k, m}^{\mathrm{GG}} V^{*} \otimes \mathcal{O}(-m \delta A)\right)$ has maximal growth, i.e. is larger that $c_{k} m^{n+k r-1}$ for some $m \geqslant m_{k}$, where $c, c_{k}>0, n=\operatorname{dim} X$ and $r=\operatorname{rank} V$. In particular, entire curves $f:\left(\mathbb{C}, T_{\mathbb{C}}\right) \rightarrow(X, V)$ satisfy (many) algebraic differential equations.

The statement is very elementary to check when $r=\operatorname{rank} V=1$, and therefore when $n=$ $\operatorname{dim} X=1$. In higher dimensions $n \geqslant 2$, only very partial results were known before Theorem 9.1 was obtained in [Dem11], [and they dealt merely with the absolute case $V=T_{X}$ ]. In dimension 2, Theorem 9.1 is a consequence of the Riemann-Roch calculation of Green-Griffiths [GrGr80], combined with a vanishing theorem due to Bogomolov [Bog79] - the latter actually only applies to the top cohomology group $H^{n}$, and things become much more delicate when extimates of intermediate cohomology groups are needed. In higher dimensions, Diverio [Div08, Div09] proved the existence of sections of $H^{0}\left(X, E_{k, m}^{\mathrm{GG}} V^{*} \otimes \mathcal{O}(-1)\right)$ whenever $X$ is a hypersurface of $\mathbb{P}_{\mathbb{C}}^{n+1}$ of high degree $d \geqslant d_{n}$, assuming $k \geqslant n$ and $m \geqslant m_{n}$. More recently, Merker [Mer15] was able to treat the case of arbitrary hypersurfaces of general type, i.e. $d \geqslant n+3$, assuming this time $k$ to be very large. The latter result is obtained through explicit algebraic calculations of the spaces of sections, and the proof is computationally very intensive. Bérczi [Ber15, Ber18] also obtained related results with a different approach based on residue formulas, assuming e.g. $d \geqslant n^{9 n}$.

All these approaches are algebraic in nature. Here, however, our techniques are based on more elaborate curvature estimates in the spirit of Cowen-Griffiths [CoGr76]. They require holomorphic Morse inequalities (see 9.10 below) - and we do not know how to translate our method in an algebraic setting. Notice that holomorphic Morse inequalities are essentially insensitive to singularities, as we can pass to non-singular models and blow-up $X$ as much as we want: if $\mu: \widetilde{X} \rightarrow X$ is a modification then $\mu_{*} \mathcal{O}_{\tilde{X}}=\mathcal{O}_{X}$ and for $q \geqslant 1, R^{q} \mu_{*} \mathcal{O}_{\tilde{X}}$ is supported on a codimension 1 analytic subset (even a codimension 2 subset if $X$ is smooth). It follows from the Leray spectral sequence that the cohomology estimates for $L$ on $X$ or for $\widetilde{L}=\mu^{*} L$ on $\widetilde{X}$ differ by negligible terms, i.e.

$$
h^{q}\left(\widetilde{X}, \widetilde{L}^{\otimes m}\right)-h^{q}\left(X, L^{\otimes m}\right)=O\left(m^{n-1}\right) .
$$

Finally, singular holomorphic Morse inequalities (in the form obtained by L. Bonavero [Bon93]) allow us to work with singular Hermitian metrics $h$; this is the reason why we will only require to have big line bundles rather than ample line bundles. In the case of linear subspaces $V \subset T_{X}$, we introduce singular Hermitian metrics as follows.

9.3. Definition. A singular Hermitian metric on a linear subspace $V \subset T_{X}$ is a metric $h$ on the fibers of $V$ such that the function $\log h: \xi \mapsto \log |\xi|_{h}^{2}$ is locally integrable on the total space of $V$.

Such a metric can also be viewed as a singular Hermitian metric on the tautological line bundle $\mathcal{O}_{P(V)}(-1)$ on the projectivized bundle $P(V)=V \backslash\{0\} / \mathbb{C}^{*}$, and therefore its dual metric $h^{*}$ defines a curvature current $\Theta_{\mathcal{O}_{P(V)}(1), h^{*}}$ of type $(1,1)$ on $P(V) \subset P\left(T_{X}\right)$, such that

$$
p^{*} \Theta_{\mathcal{O}_{P(V)}(1), h^{*}}=\frac{i}{2 \pi} \partial \bar{\partial} \log h, \quad \text { where } p: V \backslash\{0\} \rightarrow P(V) .
$$

If $\log h$ is quasi-plurisubharmonic (or quasi-psh, which means psh modulo addition of a smooth function) on $V$, then $\log h$ is indeed locally integrable, and we have moreover

$$
\Theta_{\mathcal{O}_{P(V)}(1), h^{*}} \geqslant-C \omega
$$

for some smooth positive (1,1)-form on $P(V)$ and some constant $C>0$; conversely, if (9.5) holds, then $\log h$ is quasi-psh.

9.6. Definition. We will say that a singular Hermitian metric $h$ on $V$ is admissible if $h$ can be written as $h=e^{\varphi} h_{0 \mid V}$ where $h_{0}$ is a smooth positive definite Hermitian on $T_{X}$ and $\varphi$ is a quasi-psh weight with analytic singularities on $X$, as in Definition 9.3. Then $h$ can be seen as a singular Hermitian metric on $\mathcal{O}_{P(V)}(1)$, with the property that it induces a smooth positive definite metric 
on a Zariski open set $X^{\prime} \subset X \backslash \operatorname{Sing}(V)$; we will denote by $\operatorname{Sing}(h)$ the complement of the largest such Zariski open set $X^{\prime}$ (so that $\operatorname{Sing}(h) \supset \operatorname{Sing}(V)$ ).

If $h$ is an admissible metric on $V$, we define $\mathcal{O}_{h}\left(V^{*}\right)$ to be the sheaf of germs of holomorphic sections of $V_{\lceil X \backslash \operatorname{Sing}(h)}^{*}$ which are $h^{*}$-bounded near $\operatorname{Sing}(h)$; by the assumption on the analytic singularities, this is a coherent sheaf (as the direct image of some coherent sheaf on $P(V)$ ), and actually, since $h^{*}=e^{-\varphi} h_{0}^{*}$, it is a subsheaf of the sheaf $\mathcal{O}\left(V^{*}\right):=\mathcal{O}_{h_{0}}\left(V^{*}\right)$ associated with a smooth positive definite metric $h_{0}$ on $T_{X}$. If $r$ is the generic rank of $V$ and $m$ a positive integer, we define similarly

$$
\begin{aligned}
{ }^{b} K_{V, h}^{[m]}= & \text { sheaf of germs of holomorphic sections of }\left(\operatorname{det} V_{\uparrow X^{\prime}}^{*}\right)^{\otimes m}=\left(\Lambda^{r} V_{\left\lceil X^{\prime}\right.}^{*}\right)^{\otimes m} \\
& \text { which are } \operatorname{det} h^{*} \text {-bounded, }
\end{aligned}
$$

so that ${ }^{b} K_{V}^{[m]}:={ }^{b} K_{V, h_{0}}^{[m]}$ according to Def. 2.7. For a given admissible Hermitian structure $(V, h)$, we define similarly the sheaf $E_{k, m}^{\mathrm{GG}} V_{h}^{*}$ to be the sheaf of polynomials defined over $X \backslash \operatorname{Sing}(h)$ which are " $h$-bounded". This means that when they are viewed as polynomials $P\left(z ; \xi_{1}, \ldots, \xi_{k}\right)$ in terms of $\xi_{j}=\left(\nabla_{h_{0}}^{1,0}\right)^{j} f(0)$ where $\nabla_{h_{0}}^{1,0}$ is the $(1,0)$-component of the induced Chern connection on $\left(V, h_{0}\right)$, there is a uniform bound

$$
\left|P\left(z ; \xi_{1}, \ldots, \xi_{k}\right)\right| \leqslant C\left(\sum\left\|\xi_{j}\right\|_{h}^{1 / j}\right)^{m}
$$

near points of $X \backslash X^{\prime}$ (see section 2 for more details on this). Again, by a direct image argument, one sees that $E_{k, m}^{\mathrm{GG}} V_{h}^{*}$ is always a coherent sheaf. The sheaf $E_{k, m}^{\mathrm{GG}} V^{*}$ is defined to be $E_{k, m}^{\mathrm{GG}} V_{h}^{*}$ when $h=h_{0}$ (it is actually independent of the choice of $h_{0}$, as follows from arguments similar to those given in section 2). Notice that this is exactly what is needed to extend the proof of the vanishing theorem 8.15 to the case of a singular linear space $V$; the value distribution theory argument can only work when the functions $P\left(f ; f^{\prime}, \ldots, f^{(k)}\right)(t)$ do not exhibit poles, and this is guaranteed here by the boundedness assumption.

Our strategy can be described as follows. We consider the Green-Griffiths bundle of $k$-jets $X_{k}^{\mathrm{GG}}=J^{k} V \backslash\{0\} / \mathbb{C}^{*}$, which by $(7.7)$ consists of a fibration in weighted projective spaces, and its associated tautological sheaf

$$
L=\mathcal{O}_{X_{k}^{\mathrm{GG}}}(1),
$$

viewed rather as a virtual $\mathbb{Q}$-line bundle $\mathcal{O}_{X_{k}^{G G}}\left(m_{0}\right)^{1 / m_{0}}$ with $m_{0}=\operatorname{lcm}(1,2, \ldots, k)$. Then, if $\pi_{k}: X_{k}^{\mathrm{GG}} \rightarrow X$ is the natural projection, we have

$$
E_{k, m}^{\mathrm{GG}}=\left(\pi_{k}\right)_{*} \mathcal{O}_{X_{k}^{\mathrm{GG}}}(m) \text { and } R^{q}\left(\pi_{k}\right)_{*} \mathcal{O}_{X_{k}^{\mathrm{GG}}}(m)=0 \text { for } q \geqslant 1 .
$$

Hence, by the Leray spectral sequence we get for every invertible sheaf $F$ on $X$ the isomorphism

$$
H^{q}\left(X, E_{k, m}^{\mathrm{GG}} V^{*} \otimes F\right) \simeq H^{q}\left(X_{k}^{\mathrm{GG}}, \mathcal{O}_{X_{k}^{\mathrm{GG}}}(m) \otimes \pi_{k}^{*} F\right) .
$$

The latter group can be evaluated thanks to holomorphic Morse inequalities. Let us recall the main statement.

9.10. Holomorphic Morse inequalities ([Dem85]). Let $X$ be a compact complex manifolds, $E \rightarrow X$ a holomorphic vector bundle of rank $r$, and $(L, h)$ a Hermitian line bundle. The dimensions $h^{q}\left(X, E \otimes L^{m}\right)$ of cohomology groups of the tensor powers $E \otimes L^{m}$ satisfy the following asymptotic estimates as $m \rightarrow+\infty$ :

(WM) Weak Morse inequalities:

$$
h^{q}\left(X, E \otimes L^{m}\right) \leqslant r \frac{m^{n}}{n !} \int_{X(L, h, q)}(-1)^{q} \Theta_{L, h}^{n}+o\left(m^{n}\right),
$$

where $X(L, h, q)$ denotes the open set of points $x \in X$ at which the curvature form $\Theta_{L, h}(x)$ has signature $(q, n-q)$; 
(SM) Strong Morse inequalities:

$$
\sum_{0 \leqslant j \leqslant q}(-1)^{q-j} h^{j}\left(X, E \otimes L^{m}\right) \leqslant r \frac{m^{n}}{n !} \int_{X(L, h, \leqslant q)}(-1)^{q} \Theta_{L, h}^{n}+o\left(m^{n}\right),
$$

where $X(L, h, \leqslant q)=\bigcup_{j \leqslant q} X(L, h, j)$;

(RR) Asymptotic Riemann-Roch formula:

$$
\chi\left(X, E \otimes L^{m}\right):=\sum_{0 \leqslant j \leqslant n}(-1)^{j} h^{j}\left(X, E \otimes L^{m}\right)=r \frac{m^{n}}{n !} \int_{X} \Theta_{L, h}^{n}+o\left(m^{n}\right) .
$$

Moreover (cf. Bonavero's PhD thesis [Bon93]), if $h=e^{-\varphi}$ is a singular Hermitian metric with analytic singularities of pole set $P=\varphi^{-1}(-\infty)$, the estimates still hold provided all cohomology groups are replaced by cohomology groups $H^{q}\left(X, E \otimes L^{m} \otimes \mathcal{I}\left(h^{m}\right)\right)$ twisted with the corresponding $L^{2}$ multiplier ideal sheaves

$$
\mathcal{I}\left(h^{m}\right)=\mathcal{I}(k \varphi)=\left\{f \in \mathcal{O}_{X, x}, \quad \exists V \ni x, \int_{V}|f(z)|^{2} e^{-m \varphi(z)} d \lambda(z)<+\infty\right\},
$$

and provided the Morse integrals are computed on the regular locus of $h$, namely restricted to $X(L, h, q) \backslash \Sigma:$

$$
\int_{X(L, h, q) \backslash \Sigma}(-1)^{q} \Theta_{L, h}^{n}
$$

The special case of $9.10(\mathrm{SM})$ when $q=1$ yields a very useful criterion for the existence of sections of large multiples of $L$.

9.11. Corollary. Let $L \rightarrow X$ be a holomorphic line bundle equipped with a singular Hermitian metric $h=e^{-\varphi}$ with analytic singularities of pole set $\Sigma=\varphi^{-1}(-\infty)$. Then we have the following lower bounds

(a) at the $h^{0}$ level:

$$
\begin{aligned}
h^{0}\left(X, E \otimes L^{m}\right) & \geqslant h^{0}\left(X, E \otimes L^{m} \otimes \mathcal{I}\left(h^{m}\right)\right) \\
& \geqslant h^{0}\left(X, E \otimes L^{m} \otimes \mathcal{I}\left(h^{m}\right)\right)-h^{1}\left(X, E \otimes L^{m} \otimes \mathcal{I}\left(h^{m}\right)\right) \\
& \geqslant r \frac{k^{n}}{n !} \int_{X(L, h, \leqslant 1) \backslash \Sigma} \Theta_{L, h}^{n}-o\left(k^{n}\right) .
\end{aligned}
$$

Especially $L$ is big as soon as $\int_{X(L, h, \leqslant 1) \backslash \Sigma} \Theta_{L, h}^{n}>0$ for some singular Hermitian metric $h$ on $L$. (b) at the $h^{q}$ level:

$$
h^{q}\left(X, E \otimes L^{m} \otimes \mathcal{I}\left(h^{m}\right)\right) \geqslant r \frac{k^{n}}{n !} \sum_{j=q-1, q, q+1}(-1)^{q} \int_{X(L, h, j) \backslash \Sigma} \Theta_{L, h}^{n}-o\left(k^{n}\right) .
$$

Now, given a directed manifold $(X, V)$, we can associate with any admissible metric $h$ on $V$ a metric (or rather a natural family) of metrics on $L=\mathcal{O}_{X_{k}^{\mathrm{GG}}}(1)$. The space $X_{k}^{\mathrm{GG}}$ always possesses quotient singularities if $k \geqslant 2$ (and even some more if $V$ is singular), but we do not really care since Morse inequalities still work in this setting thanks to Bonavero's generalization. As we will see, it is then possible to get nice asymptotic formulas as $m \rightarrow+\infty$. They appear to be of a probabilistic nature if we take the components of the $k$-jet (i.e. the successive derivatives $\xi_{j}=f^{(j)}(0)$, $1 \leqslant j \leqslant k$ ) as random variables. This probabilistic behaviour was somehow already visible in the Riemann-Roch calculation of [GrGr80]. In this way, assuming $K_{V}$ big, we produce a lot of sections $\sigma_{j}=H^{0}\left(X_{k}^{\mathrm{GG}}, \mathcal{O}_{X_{k}^{\mathrm{GG}}}(m) \otimes \pi_{k}^{*} F\right)$, corresponding to certain divisors $Z_{j} \subset X_{k}^{\mathrm{GG}}$. The hard problem which is left in order to complete a proof of the generalized Green-Griffiths-Lang conjecture is to compute the base locus $Z=\bigcap Z_{j}$ and to show that $Y=\pi_{k}(Z) \subset X$ must be a proper algebraic variety. 


\section{B. Hermitian GeOMETRY of Weighted PROJECTIVE SPACES}

The goal of this section is to introduce natural Kähler metrics on weighted projective spaces, and to evaluate the corresponding volume forms. Here we put $d^{c}=\frac{i}{4 \pi}(\bar{\partial}-\partial)$ so that $d d^{c}=\frac{i}{2 \pi} \partial \bar{\partial}$. The normalization of the $d^{c}$ operator is chosen such that we have precisely $\left(d d^{c} \log |z|^{2}\right)^{n}=\delta_{0}$ (the Dirac mass at 0$)$ for the Monge-Ampère operator in $\mathbb{C}^{n}$. Given a $k$-tuple of "weights" $a=\left(a_{1}, \ldots, a_{k}\right)$, i.e. of integers $a_{s}>0$ with $\operatorname{gcd}\left(a_{1}, \ldots, a_{k}\right)=1$, we introduce the weighted projective space $P\left(a_{1}, \ldots, a_{k}\right)$ to be the quotient of $\mathbb{C}^{k} \backslash\{0\}$ by the corresponding weighted $\mathbb{C}^{*}$ action:

$$
P\left(a_{1}, \ldots, a_{k}\right)=\mathbb{C}^{k} \backslash\{0\} / \mathbb{C}^{*}, \quad \lambda \cdot z=\left(\lambda^{a_{1}} z_{1}, \ldots, \lambda^{a_{k}} z_{k}\right), \quad \lambda \in \mathbb{C}^{*} .
$$

As is well known, this defines a toric $(k-1)$-dimensional algebraic variety with quotient singularities. On this variety, we introduce the possibly singular (but almost everywhere smooth and non-degenerate) Kähler form $\omega_{a, p}$ defined by

$$
\pi_{a}^{*} \omega_{a, p}=d d^{c} \varphi_{a, p}, \quad \varphi_{a, p}(z)=\frac{1}{p} \log \sum_{1 \leqslant s \leqslant k}\left|z_{s}\right|^{2 p / a_{s}}
$$

where $\pi_{a}: \mathbb{C}^{k} \backslash\{0\} \rightarrow P\left(a_{1}, \ldots, a_{k}\right)$ is the canonical projection and $p>0$ is a positive constant. It is clear that $\varphi_{p, a}$ is real analytic on $\mathbb{C}^{k} \backslash\{0\}$ if $p$ is an integer and a common multiple of all weights $a_{s}$, and we will implicitly pick such a $p$ later on to avoid any difficulty. Elementary calculations give the following well-known formula for the volume

$$
\int_{P\left(a_{1}, \ldots, a_{k}\right)} \omega_{a, p}^{k-1}=\frac{1}{a_{1} \ldots a_{k}}
$$

(notice that this is independent of $p$, as it is obvious by Stokes theorem, since the cohomology class of $\omega_{a, p}$ does not depend on $p$ ).

Our later calculations will require a slightly more general setting. Instead of looking at $\mathbb{C}^{k}$, we consider the weighted $\mathbb{C}^{*}$ action defined by

$$
\mathbb{C}^{|r|}=\mathbb{C}^{r_{1}} \times \ldots \times \mathbb{C}^{r_{k}}, \quad \lambda \cdot z=\left(\lambda^{a_{1}} z_{1}, \ldots, \lambda^{a_{k}} z_{k}\right), \quad \lambda \in \mathbb{C}^{*} .
$$

Here $z_{s} \in \mathbb{C}^{r_{s}}$ for some $k$-tuple $r=\left(r_{1}, \ldots, r_{k}\right)$ and $|r|=r_{1}+\ldots+r_{k}$. This gives rise to a weighted projective space

$$
\begin{aligned}
& P\left(a_{1}^{\left[r_{1}\right]}, \ldots, a_{k}^{\left[r_{k}\right]}\right)=P\left(a_{1}, \ldots, a_{1}, \ldots, a_{k}, \ldots, a_{k}\right), \\
& \pi_{a, r}: \mathbb{C}^{r_{1}} \times \ldots \times \mathbb{C}^{r_{k}} \backslash\{0\} \longrightarrow P\left(a_{1}^{\left[r_{1}\right]}, \ldots, a_{k}^{\left[r_{k}\right]}\right),
\end{aligned}
$$

obtained by repeating $r_{s}$ times each weight $a_{s}$. On this space, we introduce the degenerate Kähler metric $\omega_{a, r, p}$ such that

$$
\pi_{a, r}^{*} \omega_{a, r, p}=d d^{c} \varphi_{a, r, p}, \quad \varphi_{a, r, p}(z)=\frac{1}{p} \log \sum_{1 \leqslant s \leqslant k}\left|z_{s}\right|^{2 p / a_{s}},
$$

where $\left|z_{s}\right|$ stands now for the standard Hermitian norm $\left(\sum_{1 \leqslant j \leqslant r_{s}}\left|z_{s, j}\right|^{2}\right)^{1 / 2}$ on $\mathbb{C}^{r_{s}}$. This metric is cohomologous to the corresponding "polydisc-like" metric $\omega_{a, p}$ already defined, and therefore Stokes theorem implies

$$
\int_{P\left(a_{1}^{\left[r_{1}\right]}, \ldots, a_{k}^{\left[r_{k}\right]}\right)} \omega_{a, r, p}^{|r|-1}=\frac{1}{a_{1}^{r_{1}} \ldots a_{k}^{r_{k}}}
$$

Using standard results of integration theory (Fubini, change of variable formula...), one obtains:

9.19. Proposition. Let $f(z)$ be a bounded function on $P\left(a_{1}^{\left[r_{1}\right]}, \ldots, a_{k}^{\left[r_{k}\right]}\right)$ which is continuous outside of the hyperplane sections $z_{s}=0$. We also view $f$ as $a \mathbb{C}^{*}$-invariant continuous function 


$$
\begin{aligned}
& \text { on } \prod\left(\mathbb{C}^{r_{s}} \backslash\{0\}\right) \text {. Then } \\
& \qquad \int_{P\left(a_{1}^{\left[r_{1}\right]}, \ldots, a_{k}^{\left[r_{k}\right]}\right)} f(z) \omega_{a, r, p}^{|r|-1} \\
& \quad=\frac{(|r|-1) !}{\prod_{s} a_{s}^{r_{s}}} \int_{(x, u) \in \Delta_{k-1} \times \prod S^{2 r_{s}-1}} f\left(x_{1}^{a_{1} / 2 p} u_{1}, \ldots, x_{k}^{a_{k} / 2 p} u_{k}\right) \prod_{1 \leqslant s \leqslant k} \frac{x_{s}^{r_{s}-1}}{\left(r_{s}-1\right) !} d x d \mu(u)
\end{aligned}
$$

where $\Delta_{k-1}$ is the $(k-1)$-simplex $\left\{x_{s} \geqslant 0, \sum x_{s}=1\right\}, d x=d x_{1} \wedge \ldots \wedge d x_{k-1}$ its standard measure, and where $d \mu(u)=d \mu_{1}\left(u_{1}\right) \ldots d \mu_{k}\left(u_{k}\right)$ is the rotation invariant probability measure on the product $\prod_{s} S^{2 r_{s}-1}$ of unit spheres in $\mathbb{C}^{r_{1}} \times \ldots \times \mathbb{C}^{r_{k}}$. As a consequence

$$
\lim _{p \rightarrow+\infty} \int_{P\left(a_{1}^{\left[r_{1}\right]}, \ldots, a_{k}^{\left[r_{k}\right]}\right)} f(z) \omega_{a, r, p}^{|r|-1}=\frac{1}{\prod_{s} a_{s}^{r_{s}}} \int_{\prod S^{2 r_{s}-1}} f(u) d \mu(u) .
$$

Also, by elementary integrations by parts and induction on $k, r_{1}, \ldots, r_{k}$, it can be checked that

$$
\int_{x \in \Delta_{k-1}} \prod_{1 \leqslant s \leqslant k} x_{s}^{r_{s}-1} d x_{1} \ldots d x_{k-1}=\frac{1}{(|r|-1) !} \prod_{1 \leqslant s \leqslant k}\left(r_{s}-1\right) !
$$

This implies that $(|r|-1) ! \prod_{1 \leqslant s \leqslant k} \frac{x_{s}^{r_{s}-1}}{\left(r_{s}-1\right) !} d x$ is a probability measure on $\Delta_{k-1}$.

\section{C. Probabilistic estimate of the Curvature of $k$-Jet Bundles}

Let $(X, V)$ be a compact complex directed non-singular variety. To avoid any technical difficulty at this point, we first assume that $V$ is a holomorphic vector subbundle of $T_{X}$, equipped with a smooth Hermitian metric $h$.

According to the notation already specified in $\S 7$, we denote by $J^{k} V$ the bundle of $k$-jets of holomorphic curves $f:(\mathbb{C}, 0) \rightarrow X$ tangent to $V$ at each point. Let us set $n=\operatorname{dim}_{\mathbb{C}} X$ and $r=\operatorname{rank}_{\mathbb{C}} V$. Then $J^{k} V \rightarrow X$ is an algebraic fiber bundle with typical fiber $\mathbb{C}^{r k}$, and we get a projectivized $k$-jet bundle

$$
X_{k}^{\mathrm{GG}}:=\left(J^{k} V \backslash\{0\}\right) / \mathbb{C}^{*}, \quad \pi_{k}: X_{k}^{\mathrm{GG}} \rightarrow X,
$$

which is a $P\left(1^{[r]}, 2^{[r]}, \ldots, k^{[r]}\right)$ weighted projective bundle over $X$, and we have the direct image formula $\left(\pi_{k}\right)_{*} \mathcal{O}_{X_{k}^{\mathrm{GG}}}(m)=\mathcal{O}\left(E_{k, m}^{\mathrm{GG}} V^{*}\right)$ (cf. Proposition 7.9). In the sequel, we do not make a direct use of coordinates, because they need not be related in any way to the Hermitian metric $h$ of $V$. Instead, we choose a local holomorphic coordinate frame $\left(e_{\alpha}(z)\right)_{1 \leqslant \alpha \leqslant r}$ of $V$ on a neighborhood $U$ of $x_{0}$, such that

$$
\left\langle e_{\alpha}(z), e_{\beta}(z)\right\rangle=\delta_{\alpha \beta}+\sum_{1 \leqslant i, j \leqslant n, 1 \leqslant \alpha, \beta \leqslant r} c_{i j \alpha \beta} z_{i} \bar{z}_{j}+O\left(|z|^{3}\right)
$$

for suitable complex coefficients $\left(c_{i j \alpha \beta}\right)$. It is a standard fact that such a normalized coordinate system always exists, and that the Chern curvature tensor $\frac{i}{2 \pi} D_{V, h}^{2}$ of $(V, h)$ at $x_{0}$ is then given by

$$
\Theta_{V, h}\left(x_{0}\right)=-\frac{i}{2 \pi} \sum_{i, j, \alpha, \beta} c_{i j \alpha \beta} d z_{i} \wedge d \bar{z}_{j} \otimes e_{\alpha}^{*} \otimes e_{\beta} .
$$

Consider a local holomorphic connection $\nabla$ on $V_{\uparrow U}$ (e.g. the one which turns $\left(e_{\alpha}\right)$ into a parallel frame), and take $\xi_{k}=\nabla^{k} f(0) \in V_{x}$ defined inductively by $\nabla^{1} f=f^{\prime}$ and $\nabla^{s} f=\nabla_{f^{\prime}}\left(\nabla^{s-1} f\right)$. This gives a local identification

$$
J_{k} V_{\uparrow U} \rightarrow V_{\uparrow U}^{\oplus k}, \quad f \mapsto\left(\xi_{1}, \ldots, \xi_{k}\right)=\left(\nabla f(0), \ldots, \nabla f^{k}(0)\right),
$$

and the weighted $\mathbb{C}^{*}$ action on $J_{k} V$ is expressed in this setting by

$$
\lambda \cdot\left(\xi_{1}, \xi_{2}, \ldots, \xi_{k}\right)=\left(\lambda \xi_{1}, \lambda^{2} \xi_{2}, \ldots, \lambda^{k} \xi_{k}\right) .
$$

Now, we fix a finite open covering $\left(U_{\alpha}\right)_{\alpha \in I}$ of $X$ by open coordinate charts such that $V_{\uparrow U_{\alpha}}$ is trivial, along with holomorphic connections $\nabla_{\alpha}$ on $V_{\uparrow U_{\alpha}}$. Let $\theta_{\alpha}$ be a partition of unity of $X$ subordinate 
to the covering $\left(U_{\alpha}\right)$. Let us fix $p>0$ and small parameters $1=\varepsilon_{1} \gg \varepsilon_{2} \gg \ldots \gg \varepsilon_{k}>0$. Then we define a global weighted Finsler metric on $J^{k} V$ by putting for any $k$-jet $f \in J_{x}^{k} V$

$$
\Psi_{h, p, \varepsilon}(f):=\left(\sum_{\alpha \in I} \theta_{\alpha}(x) \sum_{1 \leqslant s \leqslant k} \varepsilon_{s}^{2 p}\left\|\nabla_{\alpha}^{s} f(0)\right\|_{h(x)}^{2 p / s}\right)^{1 / p},
$$

where \|\|$_{h(x)}$ is the Hermitian metric $h$ of $V$ evaluated on the fiber $V_{x}, x=f(0)$. The function $\Psi_{h, p, \varepsilon}$ satisfies the fundamental homogeneity property

$$
\Psi_{h, p, \varepsilon}(\lambda \cdot f)=\Psi_{h, p, \varepsilon}(f)|\lambda|^{2}
$$

with respect to the $\mathbb{C}^{*}$ action on $J^{k} V$, in other words, it induces a Hermitian metric on the dual $L^{*}$ of the tautological $\mathbb{Q}$-line bundle $L_{k}=\mathcal{O}_{X_{k}^{G G}}(1)$ over $X_{k}^{\mathrm{GG}}$. The curvature of $L_{k}$ is given by

$$
\pi_{k}^{*} \Theta_{L_{k}, \Psi_{h, p, \varepsilon}^{*}}=d d^{c} \log \Psi_{h, p, \varepsilon} .
$$

Our next goal is to compute precisely the curvature and to apply holomorphic Morse inequalities to $L \rightarrow X_{k}^{\mathrm{GG}}$ with the above metric. It might look a priori like an untractable problem, since the definition of $\Psi_{h, p, \varepsilon}$ is a rather unnatural one. However, the "miracle" is that the asymptotic behavior of $\Psi_{h, p, \varepsilon}$ as $\varepsilon_{s} / \varepsilon_{s-1} \rightarrow 0$ is in some sense uniquely defined and very natural. It will lead to a computable asymptotic formula, which is moreover simple enough to produce useful results.

9.27. Lemma. On each coordinate chart $U$ equipped with a holomorphic connection $\nabla$ of $V_{\uparrow U}$, let us define the components of a $k$-jet $f \in J^{k} V$ by $\xi_{s}=\nabla^{s} f(0)$, and consider the rescaling transformation

$$
\rho_{\nabla, \varepsilon}\left(\xi_{1}, \xi_{2}, \ldots, \xi_{k}\right)=\left(\varepsilon_{1}^{1} \xi_{1}, \varepsilon_{2}^{2} \xi_{2}, \ldots, \varepsilon_{k}^{k} \xi_{k}\right) \quad \text { on } J_{x}^{k} V, x \in U
$$

(it commutes with the $\mathbb{C}^{*}$-action but is otherwise unrelated and not canonically defined over $X$ as it depends on the choice of $\nabla)$. Then, if $p$ is a multiple of $\operatorname{lcm}(1,2, \ldots, k)$ and $\varepsilon_{s} / \varepsilon_{s-1} \rightarrow 0$ for all $s=2, \ldots, k$, the rescaled function $\Psi_{h, p, \varepsilon} \circ \rho_{\nabla, \varepsilon}^{-1}\left(\xi_{1}, \ldots, \xi_{k}\right)$ converges towards

$$
\left(\sum_{1 \leqslant s \leqslant k}\left\|\xi_{s}\right\|_{h}^{2 p / s}\right)^{1 / p}
$$

on every compact subset of $J^{k} V_{\uparrow U} \backslash\{0\}$, uniformly in $C^{\infty}$ topology.

Proof. Let $U \subset X$ be an open set on which $V_{\uparrow U}$ is trivial and equipped with some holomorphic connection $\nabla$. Let us pick another holomorphic connection $\widetilde{\nabla}=\nabla+\Gamma$ where $\Gamma \in H^{0}\left(U, \Omega_{X}^{1} \otimes\right.$ $\operatorname{Hom}(V, V))$. Then $\widetilde{\nabla}^{2} f=\nabla^{2} f+\Gamma(f)\left(f^{\prime}\right) \cdot f^{\prime}$, and inductively we get

$$
\widetilde{\nabla}^{s} f=\nabla^{s} f+P_{s}\left(f ; \nabla^{1} f, \ldots, \nabla^{s-1} f\right)
$$

where $P\left(x ; \xi_{1}, \ldots, \xi_{s-1}\right)$ is a polynomial of weighted degree $s$ in $\left(\xi_{1}, \ldots, \xi_{s-1}\right)$ with holomorphic coefficients in $x \in U$. In other words, the corresponding change in the parametrization of $J^{k} V_{\uparrow U}$ is given by a $\mathbb{C}^{*}$-homogeneous transformation

$$
\widetilde{\xi}_{s}=\xi_{s}+P_{s}\left(x ; \xi_{1}, \ldots, \xi_{s-1}\right) .
$$

Let us introduce the corresponding rescaled components

$$
\left(\xi_{1, \varepsilon}, \ldots, \xi_{k, \varepsilon}\right)=\left(\varepsilon_{1}^{1} \xi_{1}, \ldots, \varepsilon_{k}^{k} \xi_{k}\right), \quad\left(\widetilde{\xi}_{1, \varepsilon}, \ldots, \widetilde{\xi}_{k, \varepsilon}\right)=\left(\varepsilon_{1}^{1} \widetilde{\xi}_{1}, \ldots, \varepsilon_{k}^{k} \widetilde{\xi}_{k}\right) .
$$

Then

$$
\begin{aligned}
\widetilde{\xi}_{s, \varepsilon} & =\xi_{s, \varepsilon}+\varepsilon_{s}^{s} P_{s}\left(x ; \varepsilon_{1}^{-1} \xi_{1, \varepsilon}, \ldots, \varepsilon_{s-1}^{-(s-1)} \xi_{s-1, \varepsilon}\right) \\
& =\xi_{s, \varepsilon}+O\left(\varepsilon_{s} / \varepsilon_{s-1}\right)^{s} O\left(\left\|\xi_{1, \varepsilon}\right\|+\ldots+\left\|\xi_{s-1, \varepsilon}\right\|^{1 /(s-1)}\right)^{s}
\end{aligned}
$$


and the error terms are thus polynomials of fixed degree with arbitrarily small coefficients as $\varepsilon_{s} / \varepsilon_{s-1} \rightarrow 0$. Now, the definition of $\Psi_{h, p, \varepsilon}$ consists of glueing the sums

$$
\sum_{1 \leqslant s \leqslant k} \varepsilon_{s}^{2 p}\left\|\xi_{k}\right\|_{h}^{2 p / s}=\sum_{1 \leqslant s \leqslant k}\left\|\xi_{k, \varepsilon}\right\|_{h}^{2 p / s}
$$

corresponding to $\xi_{k}=\nabla_{\alpha}^{s} f(0)$ by means of the partition of unity $\sum \theta_{\alpha}(x)=1$. We see that by using the rescaled variables $\xi_{s, \varepsilon}$ the changes occurring when replacing a connection $\nabla_{\alpha}$ by an alternative one $\nabla_{\beta}$ are arbitrary small in $C^{\infty}$ topology, with error terms uniformly controlled in terms of the ratios $\varepsilon_{s} / \varepsilon_{s-1}$ on all compact subsets of $V^{k} \backslash\{0\}$. This shows that in $C^{\infty}$ topology, $\Psi_{h, p, \varepsilon} \circ \rho_{\nabla, \varepsilon}^{-1}\left(\xi_{1}, \ldots, \xi_{k}\right)$ converges uniformly towards $\left(\sum_{1 \leqslant s \leqslant k}\left\|\xi_{k}\right\|_{h}^{2 p / s}\right)^{1 / p}$, whatever the trivializing open set $U$ and the holomorphic connection $\nabla$ used to evaluate the components and to perform the rescaling are.

Now, we fix a point $x_{0} \in X$ and a local holomorphic frame $\left(e_{\alpha}(z)\right)_{1 \leqslant \alpha \leqslant r}$ satisfying (9.22) on a neighborhood $U$ of $x_{0}$. We introduce the rescaled components $\xi_{s}=\varepsilon_{s}^{s} \nabla^{s} f(0)$ on $J^{k} V_{\uparrow U}$ and compute the curvature of

$$
\Psi_{h, p, \varepsilon} \circ \rho_{\nabla, \varepsilon}^{-1}\left(z ; \xi_{1}, \ldots, \xi_{k}\right) \simeq\left(\sum_{1 \leqslant s \leqslant k}\left\|\xi_{s}\right\|_{h}^{2 p / s}\right)^{1 / p},
$$

(by Lemma 9.27, the errors can be taken arbitrary small in $C^{\infty}$ topology). We write $\xi_{s}=$ $\sum_{1 \leqslant \alpha \leqslant r} \xi_{s \alpha} e_{\alpha}$. By (9.22) we have

$$
\left\|\xi_{s}\right\|_{h}^{2}=\sum_{\alpha}\left|\xi_{s \alpha}\right|^{2}+\sum_{i, j, \alpha, \beta} c_{i j \alpha \beta} z_{i} \bar{z}_{j} \xi_{s \alpha} \bar{\xi}_{s \beta}+O\left(|z|^{3}|\xi|^{2}\right) .
$$

The question is to evaluate the curvature of the weighted metric defined by

$$
\begin{aligned}
\Psi\left(z ; \xi_{1}, \ldots, \xi_{k}\right) & =\left(\sum_{1 \leqslant s \leqslant k}\left\|\xi_{s}\right\|_{h}^{2 p / s}\right)^{1 / p} \\
& =\left(\sum_{1 \leqslant s \leqslant k}\left(\sum_{\alpha}\left|\xi_{s \alpha}\right|^{2}+\sum_{i, j, \alpha, \beta} c_{i j \alpha \beta} z_{i} \bar{z}_{j} \xi_{s \alpha} \bar{\xi}_{s \beta}\right)^{p / s}\right)^{1 / p}+O\left(|z|^{3}\right) .
\end{aligned}
$$

We set $\left|\xi_{s}\right|^{2}=\sum_{\alpha}\left|\xi_{s \alpha}\right|^{2}$. A straightforward calculation yields

$$
\log \Psi\left(z ; \xi_{1}, \ldots, \xi_{k}\right)=\frac{1}{p} \log \sum_{1 \leqslant s \leqslant k}\left|\xi_{s}\right|^{2 p / s}+\sum_{1 \leqslant s \leqslant k} \frac{1}{s} \frac{\left|\xi_{s}\right|^{2 p / s}}{\sum_{t}\left|\xi_{t}\right|^{2 p / t}} \sum_{i, j, \alpha, \beta} c_{i j \alpha \beta} z_{i} \bar{z}_{j} \frac{\xi_{s \alpha} \bar{\xi}_{s \beta}}{\left|\xi_{s}\right|^{2}}+O\left(|z|^{3}\right) .
$$

By (9.26), the curvature form of $L_{k}=\mathcal{O}_{X_{k}^{G G}}(1)$ is given at the central point $x_{0}$ by the following formula.

9.28. Proposition. With the above choice of coordinates and with respect to the rescaled components $\xi_{s}=\varepsilon_{s}^{s} \nabla^{s} f(0)$ at $x_{0} \in X$, we have the approximate expression

$$
\Theta_{L_{k}, \Psi_{h, p, \varepsilon}^{*}}\left(x_{0},[\xi]\right) \simeq \omega_{a, r, p}(\xi)+\frac{i}{2 \pi} \sum_{1 \leqslant s \leqslant k} \frac{1}{s} \frac{\left|\xi_{s}\right|^{2 p / s}}{\sum_{t}\left|\xi_{t}\right|^{2 p / t}} \sum_{i, j, \alpha, \beta} c_{i j \alpha \beta} \frac{\xi_{s \alpha} \bar{\xi}_{s \beta}}{\left|\xi_{s}\right|^{2}} d z_{i} \wedge d \bar{z}_{j},
$$

where the error terms are $O\left(\max _{2 \leqslant s \leqslant k}\left(\varepsilon_{s} / \varepsilon_{s-1}\right)^{s}\right)$ uniformly on the compact variety $X_{k}^{\mathrm{GG}}$. Here $\omega_{a, r, p}$ is the (degenerate) Kähler metric associated with the weight $a=\left(1^{[r]}, 2^{[r]}, \ldots, k^{[r]}\right)$ of the canonical $\mathbb{C}^{*}$ action on $J^{k} V$.

Thanks to the uniform approximation, we can (and will) neglect the error terms in the calculations below. Since $\omega_{a, r, p}$ is positive definite on the fibers of $X_{k}^{\mathrm{GG}} \rightarrow X$ (at least outside of the 
axes $\left.\xi_{s}=0\right)$, the index of the $(1,1)$ curvature form $\Theta_{L_{k}, \Psi_{h, p, \varepsilon}^{*}}(z,[\xi])$ is equal to the index of the $(1,1)$-form

$$
\gamma_{k}(z, \xi):=\frac{i}{2 \pi} \sum_{1 \leqslant s \leqslant k} \frac{1}{s} \frac{\left|\xi_{s}\right|^{2 p / s}}{\sum_{t}\left|\xi_{t}\right|^{2 p / t}} \sum_{i, j, \alpha, \beta} c_{i j \alpha \beta}(z) \frac{\xi_{s \alpha} \bar{\xi}_{s \beta}}{\left|\xi_{s}\right|^{2}} d z_{i} \wedge d \bar{z}_{j}
$$

depending only on the differentials $\left(d z_{j}\right)_{1 \leqslant j \leqslant n}$ on $X$. The $q$-index integral of $\left(L_{k}, \Psi_{h, p, \varepsilon}^{*}\right)$ on $X_{k}^{\mathrm{GG}}$ is therefore equal to

$$
\begin{aligned}
& \int_{X_{k}^{\mathrm{GG}}\left(L_{k}, q\right)} \Theta_{L_{k}, \Psi_{h, p, \varepsilon}^{*}}^{n+k r-1} \\
& =\frac{(n+k r-1) !}{n !(k r-1) !} \int_{z \in X} \int_{\xi \in P\left(1^{[r]}, \ldots, k[r]\right)} \omega_{a, r, p}^{k r-1}(\xi) \mathbb{1}_{\gamma_{k}, q}(z, \xi) \gamma_{k}(z, \xi)^{n},
\end{aligned}
$$

where $\mathbb{1}_{\gamma_{k}, q}(z, \xi)$ is the characteristic function of the open set of points where $\gamma_{k}(z, \xi)$ has signature $(n-q, q)$ in terms of the $d z_{j}$ 's. Notice that since $\gamma_{k}(z, \xi)^{n}$ is a determinant, the product $\mathbb{1}_{\gamma_{k}, q}(z, \xi) \gamma_{k}(z, \xi)^{n}$ gives rise to a continuous function on $X_{k}^{\mathrm{GG}}$. Formula (9.20) with $r_{1}=\ldots=r_{k}=r$ and $a_{s}=s$ yields the slightly more explicit integral

$$
\begin{aligned}
& \int_{X_{k}^{\mathrm{GG}}\left(L_{k}, q\right)} \Theta_{L_{k}, \Psi_{h, p, \varepsilon}^{*}}^{n+k r-1}=\frac{(n+k r-1) !}{n !(k !)^{r}} \times \\
& \quad \int_{z \in X} \int_{(x, u) \in \Delta_{k-1} \times\left(S^{2 r-1}\right)^{k}} \mathbb{1}_{g_{k}, q}(z, x, u) g_{k}(z, x, u)^{n} \frac{\left(x_{1} \ldots x_{k}\right)^{r-1}}{(r-1) !^{k}} d x d \mu(u),
\end{aligned}
$$

where $g_{k}(z, x, u)=\gamma_{k}\left(z, x_{1}^{1 / 2 p} u_{1}, \ldots, x_{k}^{k / 2 p} u_{k}\right)$ is given by

$$
g_{k}(z, x, u)=\frac{i}{2 \pi} \sum_{1 \leqslant s \leqslant k} \frac{1}{s} x_{s} \sum_{i, j, \alpha, \beta} c_{i j \alpha \beta}(z) u_{s \alpha} \bar{u}_{s \beta} d z_{i} \wedge d \bar{z}_{j}
$$

and $\mathbb{1}_{g_{k}, q}(z, x, u)$ is the characteristic function of its $q$-index set. Here

$$
d \nu_{k, r}(x)=(k r-1) ! \frac{\left(x_{1} \ldots x_{k}\right)^{r-1}}{(r-1) !^{k}} d x
$$

is a probability measure on $\Delta_{k-1}$, and we can rewrite

$$
\begin{aligned}
\int_{X_{k}^{\mathrm{GG}}\left(L_{k}, q\right)} \Theta_{L_{k}, \Psi_{h, p, \varepsilon}^{*}}^{n+k r-1}=\frac{(n+k r-1) !}{n !(k !)^{r}(k r-1) !} \times \\
\quad \int_{z \in X} \int_{(x, u) \in \Delta_{k-1} \times\left(S^{2 r-1}\right)^{k}} \mathbb{1}_{g_{k}, q}(z, x, u) g_{k}(z, x, u)^{n} d \nu_{k, r}(x) d \mu(u) .
\end{aligned}
$$

Now, formula (9.30) shows that $g_{k}(z, x, u)$ is a "Monte Carlo" evaluation of the curvature tensor, obtained by averaging the curvature at random points $u_{s} \in S^{2 r-1}$ with certain positive weights $x_{s} / s$; we should then think of the $k$-jet $f$ as some sort of random variable such that the derivatives $\nabla^{k} f(0)$ are uniformly distributed in all directions. Let us compute the expected value of $(x, u) \mapsto$ $g_{k}(z, x, u)$ with respect to the probability measure $d \nu_{k, r}(x) d \mu(u)$. Since $\int_{S^{2 r-1}} u_{s \alpha} \bar{u}_{s \beta} d \mu\left(u_{s}\right)=\frac{1}{r} \delta_{\alpha \beta}$ and $\int_{\Delta_{k-1}} x_{s} d \nu_{k, r}(x)=\frac{1}{k}$, we find

$$
\mathbf{E}\left(g_{k}(z, \bullet, \bullet)\right)=\frac{1}{k r} \sum_{1 \leqslant s \leqslant k} \frac{1}{s} \cdot \frac{i}{2 \pi} \sum_{i, j, \alpha} c_{i j \alpha \alpha}(z) d z_{i} \wedge d \bar{z}_{j} .
$$

In other words, we get the normalized trace of the curvature, i.e.

$$
\mathbf{E}\left(g_{k}(z, \bullet, \bullet)\right)=\frac{1}{k r}\left(1+\frac{1}{2}+\ldots+\frac{1}{k}\right) \Theta_{\operatorname{det}\left(V^{*}\right), \operatorname{det} h^{*},}
$$


where $\Theta_{\operatorname{det}\left(V^{*}\right), \operatorname{det} h^{*}}$ is the $(1,1)$-curvature form of $\operatorname{det}\left(V^{*}\right)$ with the metric induced by $h$. It is natural to guess that $g_{k}(z, x, u)$ behaves asymptotically as its expected value $\mathbf{E}\left(g_{k}(z, \bullet, \bullet)\right)$ when $k$ tends to infinity. If we replace brutally $g_{k}$ by its expected value in (9.32), we get the integral

$$
\frac{(n+k r-1) !}{n !(k !)^{r}(k r-1) !} \frac{1}{(k r)^{n}}\left(1+\frac{1}{2}+\ldots+\frac{1}{k}\right)^{n} \int_{X} \mathbb{1}_{\eta, q} \eta^{n}
$$

where $\eta:=\Theta_{\operatorname{det}\left(V^{*}\right) \text {,det } h^{*}}$ and $\mathbb{1}_{\eta, q}$ is the characteristic function of its $q$-index set in $X$. The leading constant is equivalent to $(\log k)^{n} / n !(k !)^{r}$ modulo a multiplicative factor $(1+O(1 / \log k))$. By working out a more precise analysis of the deviation, the following result has been proved in [Dem11] and [Dem12].

9.34. Probabilistic estimate. Fix smooth Hermitian metrics $h$ on $V$ and $\omega=\frac{i}{2 \pi} \sum \omega_{i j} d z_{i} \wedge d \bar{z}_{j}$ on $X$. Denote by $\Theta_{V, h}=-\frac{i}{2 \pi} \sum c_{i j \alpha \beta} d z_{i} \wedge d \bar{z}_{j} \otimes e_{\alpha}^{*} \otimes e_{\beta}$ the curvature tensor of $V$ with respect to an $h$-orthonormal frame $\left(e_{\alpha}\right)$, and put

$$
\eta(z)=\Theta_{\operatorname{det}\left(V^{*}\right), \operatorname{det} h^{*}}=\frac{i}{2 \pi} \sum_{1 \leqslant i, j \leqslant n} \eta_{i j} d z_{i} \wedge d \bar{z}_{j}, \quad \eta_{i j}=\sum_{1 \leqslant \alpha \leqslant r} c_{i j \alpha \alpha} .
$$

Finally consider the $k$-jet line bundle $L_{k}=\mathcal{O}_{X_{k}^{\mathrm{GG}}}(1) \rightarrow X_{k}^{\mathrm{GG}}$ equipped with the induced metric $\Psi_{h, p, \varepsilon}^{*}$ (as defined above, with $\left.1=\varepsilon_{1} \gg \varepsilon_{2} \gg \ldots \gg \varepsilon_{k}>0\right)$. When $k$ tends to infinity, the integral of the top power of the curvature of $L_{k}$ on its q-index set $X_{k}^{\mathrm{GG}}\left(L_{k}, q\right)$ is given by

$$
\int_{X_{k}^{\mathrm{GG}}\left(L_{k}, q\right)} \Theta_{L_{k}, \Psi_{h, p, \varepsilon}^{*}}^{n+k r-1}=\frac{(\log k)^{n}}{n !(k !)^{r}}\left(\int_{X} \mathbb{1}_{\eta, q} \eta^{n}+O\left((\log k)^{-1}\right)\right)
$$

for all $q=0,1, \ldots, n$, and the error term $O\left((\log k)^{-1}\right)$ can be bounded explicitly in terms of $\Theta_{V}, \eta$ and $\omega$. Moreover, the left hand side is identically zero for $q>n$.

The final statement follows from the observation that the curvature of $L_{k}$ is positive along the fibers of $X_{k}^{\mathrm{GG}} \rightarrow X$, by the plurisubharmonicity of the weight (this is true even when the partition of unity terms are taken into account, since they depend only on the base); therefore the $q$-index sets are empty for $q>n$. It will be useful to extend the above estimates to the case of sections of

$$
L_{k}=\mathcal{O}_{X_{k}^{G G}}(1) \otimes \pi_{k}^{*} \mathcal{O}\left(\frac{1}{k r}\left(1+\frac{1}{2}+\ldots+\frac{1}{k}\right) F\right),
$$

where $F \in \operatorname{Pic}_{\mathbb{Q}}(X)$ is an arbitrary $\mathbb{Q}$-line bundle on $X$ and $\pi_{k}: X_{k}^{\mathrm{GG}} \rightarrow X$ is the natural projection. We assume here that $F$ is also equipped with a smooth Hermitian metric $h_{F}$. In formulas (9.32), (9.33) and estimate 9.34, the renormalized curvature $\eta_{k}(z, x, u)$ of $L_{k}$ takes the form

$$
\eta_{k}(z, x, u)=\frac{1}{\frac{1}{k r}\left(1+\frac{1}{2}+\ldots+\frac{1}{k}\right)} g_{k}(z, x, u)+\Theta_{F, h_{F}}(z),
$$

and by the same calculations its expected value is

$$
\eta(z):=\mathbf{E}\left(\eta_{k}(z, \bullet, \bullet)\right)=\Theta_{\operatorname{det} V^{*}, \operatorname{det} h^{*}}(z)+\Theta_{F, h_{F}}(z) .
$$

Then the variance estimate for $\eta_{k}-\eta$ is unchanged, and the $L^{p}$ bounds for $\eta_{k}$ are still valid, since our forms are just shifted by adding the constant smooth term $\Theta_{F, h_{F}}(z)$. The probabilistic estimate 9.34 is therefore still true exactly in the same form, provided we use $(9.35-9.37)$ instead of the previously defined $L_{k}, \eta_{k}$ and $\eta$. An application of holomorphic Morse inequalities gives the desired cohomology estimates for

$$
\begin{aligned}
h^{q}\left(X, E_{k, m}^{\mathrm{GG}} V^{*}\right. & \left.\otimes \mathcal{O}\left(\frac{m}{k r}\left(1+\frac{1}{2}+\ldots+\frac{1}{k}\right) F\right)\right) \\
& =h^{q}\left(X_{k}^{\mathrm{GG}}, \mathcal{O}_{X_{k}^{\mathrm{GG}}}(m) \otimes \pi_{k}^{*} \mathcal{O}\left(\frac{m}{k r}\left(1+\frac{1}{2}+\ldots+\frac{1}{k}\right) F\right)\right),
\end{aligned}
$$

provided $m$ is sufficiently divisible to give a multiple of $F$ which is a $\mathbb{Z}$-line bundle. 
9.38. Theorem. Let $(X, V)$ be a directed manifold, $F \rightarrow X$ a $\mathbb{Q}$-line bundle, $(V, h)$ and $\left(F, h_{F}\right)$ smooth Hermitian structures on $V$ and $F$ respectively. We define

$$
\begin{aligned}
L_{k} & =\mathcal{O}_{X_{k}^{\mathrm{GG}}}(1) \otimes \pi_{k}^{*} \mathcal{O}\left(\frac{1}{k r}\left(1+\frac{1}{2}+\ldots+\frac{1}{k}\right) F\right), \\
\eta & =\Theta_{\operatorname{det} V^{*}, \operatorname{det} h^{*}}+\Theta_{F, h_{F}},
\end{aligned}
$$

and let $X(\eta, q)$ be the open set of points $x \in X$ where $\eta(x)$ has signature $(q, n-q)$. We also set $X(\eta, \leqslant q)=\bigcup_{j \leqslant q} X(\eta, j)$. Then for all $q \geqslant 0$ and all $m \gg k \gg 1$ such that $m$ is sufficiently divisible, we have

$$
\begin{aligned}
h^{q}\left(X_{k}^{\mathrm{GG}}, \mathcal{O}\left(L_{k}^{\otimes m}\right)\right) \leqslant \frac{m^{n+k r-1}}{(n+k r-1) !} \frac{(\log k)^{n}}{n !(k !)^{r}}\left(\int_{X(\eta, q)}(-1)^{q} \eta^{n}+O\left((\log k)^{-1}\right)\right), \\
h^{0}\left(X_{k}^{\mathrm{GG}}, \mathcal{O}\left(L_{k}^{\otimes m}\right)\right) \geqslant \frac{m^{n+k r-1}}{(n+k r-1) !} \frac{(\log k)^{n}}{n !(k !)^{r}}\left(\int_{X(\eta, \leqslant 1)} \eta^{n}-O\left((\log k)^{-1}\right)\right), \\
\chi\left(X_{k}^{\mathrm{GG}}, \mathcal{O}\left(L_{k}^{\otimes m}\right)\right)=\frac{m^{n+k r-1}}{(n+k r-1) !} \frac{(\log k)^{n}}{n !(k !)^{r}}\left(c_{1}\left(V^{*} \otimes F\right)^{n}+O\left((\log k)^{-1}\right)\right) .
\end{aligned}
$$

Green and Griffiths [GrGr80] already checked the Riemann-Roch calculation (9.38c) in the special case $V=T_{X}^{*}$ and $F=\mathcal{O}_{X}$. Their proof is much simpler since it relies only on Chern class calculations, but it cannot provide any information on the individual cohomology groups, except in very special cases where vanishing theorems can be applied; in fact in dimension 2, the Euler characteristic satisfies $\chi=h^{0}-h^{1}+h^{2} \leqslant h^{0}+h^{2}$, and hence it is enough to get the vanishing of the top cohomology group $H^{2}$ to infer $h^{0} \geqslant \chi$; this works for surfaces by means of a well-known vanishing theorem of Bogomolov which implies in general

$$
\left.H^{n}\left(X, E_{k, m}^{\mathrm{GG}} T_{X}^{*} \otimes \mathcal{O}\left(\frac{m}{k r}\left(1+\frac{1}{2}+\ldots+\frac{1}{k}\right) F\right)\right)\right)=0
$$

as soon as $K_{X} \otimes F$ is big and $m \gg 1$.

In fact, thanks to Bonavero's singular holomorphic Morse inequalities [Bon93], everything works almost unchanged in the case where $V \subset T_{X}$ has singularities and $h$ is an admissible metric on $V$ (see Definition 9.6). We only have to find a blow-up $\mu: \widetilde{X}_{k} \rightarrow X_{k}$ so that the resulting pullbacks $\mu^{*} L_{k}$ and $\mu^{*} V$ are locally free, and $\mu^{*} \operatorname{det} h^{*}, \mu^{*} \Psi_{h, p, \varepsilon}$ only have divisorial singularities. Then $\eta$ is a $(1,1)$-current with logarithmic poles, and we have to deal with smooth metrics on $\mu^{*} L_{k}^{\otimes m} \otimes \mathcal{O}\left(-m E_{k}\right)$ where $E_{k}$ is a certain effective divisor on $X_{k}$ (which, by our assumption in Definition 9.6, does not project onto $X$ ). The cohomology groups involved are then the twisted cohomology groups

$$
H^{q}\left(X_{k}^{\mathrm{GG}}, \mathcal{O}\left(L_{k}^{\otimes m}\right) \otimes \mathcal{J}_{k, m}\right),
$$

where $\mathcal{J}_{k, m}=\mu_{*}\left(\mathcal{O}\left(-m E_{k}\right)\right)$ is the corresponding multiplier ideal sheaf, and the Morse integrals need only be evaluated in the complement of the poles, i.e., on $X(\eta, q) \backslash S$ with $S=\operatorname{Sing}(V) \cup$ $\operatorname{Sing}(h)$. Since

$$
\left.\left(\pi_{k}\right)_{*}\left(\mathcal{O}\left(L_{k}^{\otimes m}\right) \otimes \mathcal{J}_{k, m}\right) \subset E_{k, m}^{\mathrm{GG}} V^{*} \otimes \mathcal{O}\left(\frac{m}{k r}\left(1+\frac{1}{2}+\ldots+\frac{1}{k}\right) F\right)\right)
$$

we still get a lower bound for the $h^{0}$ of the latter sheaf (or for the $h^{0}$ of the un-twisted line bundle $\mathcal{O}\left(L_{k}^{\otimes m}\right)$ on $\left.X_{k}^{\mathrm{GG}}\right)$. If we assume that $K_{V} \otimes F$ is big, these considerations also allow us to obtain a strong estimate in terms of the volume, by using an approximate Zariski decomposition on a suitable blow-up of $(X, V)$. The following corollary implies Theorem 9.1 as a consequence. 
9.39. Corollary. If $F$ is an arbitrary $\mathbb{Q}$-line bundle over $X$, one has

$$
\begin{aligned}
& h^{0}\left(X_{k}^{\mathrm{GG}}, \mathcal{O}_{X_{k}^{\mathrm{GG}}}(m) \otimes \pi_{k}^{*} \mathcal{O}\left(\frac{m}{k r}\left(1+\frac{1}{2}+\ldots+\frac{1}{k}\right) F\right)\right) \\
& \geqslant \frac{m^{n+k r-1}}{(n+k r-1) !} \frac{(\log k)^{n}}{n !(k !)^{r}}\left(\operatorname{Vol}\left(K_{V} \otimes F\right)-O\left((\log k)^{-1}\right)\right)-o\left(m^{n+k r-1}\right),
\end{aligned}
$$

when $m \gg k \gg 1$, in particular there are many sections of the $k$-jet differentials of degree $m$ twisted by the appropriate power of $F$ if $K_{V} \otimes F$ is big.

Proof. The volume is computed here as usual, i.e. after performing a suitable log-resolution $\mu$ : $\widetilde{X} \rightarrow X$ which converts $K_{V}$ into an invertible sheaf. There is of course nothing to prove if $K_{V} \otimes F$ is not big, so we can assume $\operatorname{Vol}\left(K_{V} \otimes F\right)>0$. Let us fix smooth Hermitian metrics $h_{0}$ on $T_{X}$ and $h_{F}$ on $F$. They induce a metric $\mu^{*}\left(\operatorname{det}\left(h_{0}^{-1}\right) \otimes h_{F}\right)$ on $\mu^{*}\left(K_{V} \otimes F\right)$ which, by our definition of $K_{V}$, is a smooth metric (the divisor produced by the log-resolution gets simplified with the degeneration divisor of the pull-back of the quotient metric on $\operatorname{det}\left(V^{*}\right)$ induced by $\left.\mathcal{O}\left(\Lambda^{r} T_{X}^{*}\right) \rightarrow \mathcal{O}\left(\Lambda^{r} V^{*}\right)\right)$. By the result of Fujita [Fuji94] on approximate Zariski decomposition, for every $\delta>0$, one can find a modification $\mu_{\delta}: \widetilde{X}_{\delta} \rightarrow X$ dominating $\mu$ such that

$$
\mu_{\delta}^{*}\left(K_{V} \otimes F\right)=\mathcal{O}_{\widetilde{X}_{\delta}}(A+E)
$$

where $A$ and $E$ are $\mathbb{Q}$-divisors, $A$ ample and $E$ effective, with

$$
\operatorname{Vol}(A)=A^{n} \geqslant \operatorname{Vol}\left(K_{V} \otimes F\right)-\delta .
$$

If we take a smooth metric $h_{A}$ with positive definite curvature form $\Theta_{A, h_{A}}$, then we get a singular Hermitian metric $h_{A} h_{E}$ on $\mu_{\delta}^{*}\left(K_{V} \otimes F\right)$ with poles along $E$, i.e. the quotient $h_{A} h_{E} / \mu_{\delta}^{*}\left(\operatorname{det}\left(h_{0}^{-1}\right) \otimes h_{F}\right)$ is of the form $e^{-\varphi}$ where $\varphi$ is quasi-psh with $\log$ poles $\log \left|\sigma_{E}\right|^{2}\left(\bmod C^{\infty}\left(\widetilde{X}_{\delta}\right)\right)$ precisely given by the divisor $E$. We then only need to take the singular metric $h$ on $T_{X}$ defined by

$$
h=h_{0} e^{\frac{1}{r}\left(\mu_{\delta}\right)_{*} \varphi}
$$

(the choice of the factor $\frac{1}{r}$ is there to correct adequately the metric on $\operatorname{det} V$ ). By construction $h$ induces an admissible metric on $V$ and the resulting curvature current $\eta=\Theta_{K_{V} \text {, det } h^{*}}+\Theta_{F, h_{F}}$ is such that

$$
\mu_{\delta}^{*} \eta=\Theta_{A, h_{A}}+[E], \quad[E]=\text { current of integration on } E .
$$

Then the 0 -index Morse integral in the complement of the poles is given by

$$
\int_{X(\eta, 0) \backslash S} \eta^{n}=\int_{\widetilde{X}_{\delta}} \Theta_{A, h_{A}}^{n}=A^{n} \geqslant \operatorname{Vol}\left(K_{V} \otimes F\right)-\delta
$$

and Corollary 9.39 follows from the fact that $\delta$ can be taken arbitrary small.

The following corollary implies Theorem 0.12 .

9.40. Corollary. Let $(X, V)$ be a projective directed manifold such that $K_{V}^{\bullet}$ is big, and $A$ an ample $\mathbb{Q}$-divisor on $X$ such that $K_{V}^{\bullet} \otimes \mathcal{O}(-A)$ is still big. Then, if we put $r=\operatorname{rank} V$ and $\delta_{k}=\frac{1}{k r}\left(1+\frac{1}{2}+\ldots+\frac{1}{k}\right)$, the space of global invariant jet differentials

$$
H^{0}\left(X, E_{k, m} V^{*} \otimes \mathcal{O}\left(-m \delta_{k} A\right)\right)
$$

has (many) non-zero sections for $m \gg k \gg 1$ and $m$ sufficiently divisible.

Proof. Corollary 9.39 produces a non-zero section $P \in H^{0}\left(E_{k, m}^{\mathrm{GG}} V^{*} \otimes \mathcal{O}_{X}\left(-m \delta_{k} A\right)\right)$ for $m \gg k \gg 1$, and the arguments given in subsection 7.E (cf. (7.36)) yield a non-zero section

$$
Q \in H^{0}\left(E_{k, m^{\prime}} V^{*} \otimes \mathcal{O}_{X}\left(-m \delta_{k} A\right)\right), \quad m^{\prime} \leqslant m .
$$

By raising $Q$ to some power $p$ and using a section $\sigma \in H^{0}\left(X, \mathcal{O}_{X}(d A)\right)$, we obtain a section

$$
Q^{p} \sigma^{m q} \in H^{0}\left(X, E_{k, p m^{\prime}} V^{*} \otimes \mathcal{O}\left(-m\left(p \delta_{k}-q d\right) A\right)\right) .
$$


One can adjust $p$ and $q$ so that $m\left(p \delta_{k}-q d\right)=p m^{\prime} \delta_{k}$ and $p m^{\prime} \delta_{k} A$ is an integral divisor.

9.41. Example. In some simple cases, the above estimates can lead to very explicit results. Take for instance $X$ to be a smooth complete intersection of multidegree $\left(d_{1}, d_{2}, \ldots, d_{s}\right)$ in $\mathbb{P}_{\mathbb{C}}^{n+s}$ and consider the absolute case $V=T_{X}$. Then $K_{X}=\mathcal{O}_{X}\left(d_{1}+\ldots+d_{s}-n-s-1\right)$ and one can check via explicit bounds of the error terms (cf. [Dem11], [Dem12]) that a sufficient condition for the existence of sections is

$$
k \geqslant \exp \left(7.38 n^{n+\frac{1}{2}}\left(\frac{\sum d_{j}+1}{\sum d_{j}-n-s-a-1}\right)^{n}\right) .
$$

This is good in view of the fact that we can cover arbitrary smooth complete intersections of general type. On the other hand, even when the degrees $d_{j}$ tend to $+\infty$, we still get a large lower bound $k \sim \exp \left(7.38 n^{n+\frac{1}{2}}\right)$ on the order of jets, and this is far from being optimal: Diverio [Div08, Div09] has shown e.g. that one can take $k=n$ for smooth hypersurfaces of high degree, using the algebraic Morse inequalities of Trapani [Tra95]. The next paragraph uses essentially the same idea, in our more analytic setting.

\section{D. Non probabilistic estimate of the Morse integrals}

We assume here that the curvature tensor $\left(c_{i j \alpha \beta}\right)$ satisfies a lower bound

$$
\sum_{i, j, \alpha, \beta} c_{i j \alpha \beta} \xi_{i} \bar{\xi}_{j} u_{\alpha} \bar{u}_{\beta} \geqslant-\sum \gamma_{i j} \xi_{i} \bar{\xi}_{j}|u|^{2}, \quad \forall \xi \in T_{X}, u \in V
$$

for some semi-positive $(1,1)$-form $\gamma=\frac{i}{2 \pi} \sum \gamma_{i j}(z) d z_{i} \wedge d \bar{z}_{j}$ on $X$. This is the same as assuming that the curvature tensor of $\left(V^{*}, h^{*}\right)$ satisfies the semi-positivity condition

$$
\Theta_{V^{*}, h^{*}}+\gamma \otimes \operatorname{Id}_{V^{*}} \geqslant 0
$$

in the sense of Griffiths, or equivalently $\Theta_{V, h}-\gamma \otimes \mathrm{Id}_{V} \leqslant 0$. Thanks to the compactness of $X$, such a form $\gamma$ always exists if $h$ is an admissible metric on $V$. Now, instead of replacing $\Theta_{V}$ with its trace free part $\widetilde{\Theta}_{V}$ and exploiting a Monte Carlo convergence process, we replace $\Theta_{V}$ with $\Theta_{V}^{\gamma}=\Theta_{V}-\gamma \otimes \operatorname{Id}_{V} \leqslant 0$, i.e. $c_{i j \alpha \beta}$ by $c_{i j \alpha \beta}^{\gamma}=c_{i j \alpha \beta}+\gamma_{i j} \delta_{\alpha \beta}$. Also, we take a line bundle $F=A^{-1}$ with $\Theta_{A, h_{A}} \geqslant$ 0, i.e. $F$ semi-negative. Then our earlier formulas in Prop. 9.28, and (9.35), (9.36) become instead

$$
\begin{aligned}
& g_{k}^{\gamma}(z, x, u)=\frac{i}{2 \pi} \sum_{1 \leqslant s \leqslant k} \frac{1}{s} x_{s} \sum_{i, j, \alpha, \beta} c_{i j \alpha \beta}^{\gamma}(z) u_{s \alpha} \bar{u}_{s \beta} d z_{i} \wedge d \bar{z}_{j} \geqslant 0, \\
& L_{k}=\mathcal{O}_{X_{k}^{G G}}(1) \otimes \pi_{k}^{*} \mathcal{O}\left(-\frac{1}{k r}\left(1+\frac{1}{2}+\ldots+\frac{1}{k}\right) A\right), \\
& \Theta_{L_{k}}=\eta_{k}(z, x, u)=\frac{1}{\frac{1}{k r}\left(1+\frac{1}{2}+\ldots+\frac{1}{k}\right)} g_{k}^{\gamma}(z, x, u)-\left(\Theta_{A, h_{A}}(z)+r \gamma(z)\right) .
\end{aligned}
$$

In fact, replacing $\Theta_{V}$ by $\Theta_{V}-\gamma \otimes \operatorname{Id}_{V}$ has the effect of replacing $\Theta_{\operatorname{det} V^{*}}=\operatorname{Tr} \Theta_{V^{*}}$ by $\Theta_{\operatorname{det} V^{*}}+r \gamma$. The major gain that we have is that $\eta_{k}=\Theta_{L_{k}}$ is now expressed as a difference of semi-positive $(1,1)$-forms, and we can exploit the following simple lemma, which is the key to derive algebraic Morse inequalities from their analytic form (cf. [Dem94], Theorem 12.3).

9.46. Lemma. Let $\eta=\alpha-\beta$ be a difference of semi-positive (1,1)-forms on an $n$-dimensional complex manifold $X$, and let $\mathbb{1}_{\eta, \leqslant q}$ be the characteristic function of the open set where $\eta$ is nondegenerate with a number of negative eigenvalues at most equal to $q$. Then

$$
(-1)^{q} \mathbb{1}_{\eta, \leqslant q} \eta^{n} \leqslant \sum_{0 \leqslant j \leqslant q}(-1)^{q-j} \alpha^{n-j} \beta^{j},
$$

in particular

$$
\mathbb{1}_{\eta, \leqslant 1} \eta^{n} \geqslant \alpha^{n}-n \alpha^{n-1} \wedge \beta \quad \text { for } q=1 .
$$


Proof. Without loss of generality, we can assume $\alpha>0$ positive definite, so that $\alpha$ can be taken as the base Hermitian metric on $X$. Let us denote by

$$
\lambda_{1} \geqslant \lambda_{2} \geqslant \ldots \geqslant \lambda_{n} \geqslant 0
$$

the eigenvalues of $\beta$ with respect to $\alpha$. The eigenvalues of $\eta=\alpha-\beta$ are then given by

$$
1-\lambda_{1} \leqslant \ldots \leqslant 1-\lambda_{q} \leqslant 1-\lambda_{q+1} \leqslant \ldots \leqslant 1-\lambda_{n}
$$

hence the open set $\left\{\lambda_{q+1}<1\right\}$ coincides with the support of $\mathbb{1}_{\eta, \leqslant q}$, except that it may also contain a part of the degeneration set $\eta^{n}=0$. On the other hand we have

$$
\left(\begin{array}{c}
n \\
j
\end{array}\right) \alpha^{n-j} \wedge \beta^{j}=\sigma_{n}^{j}(\lambda) \alpha^{n}
$$

where $\sigma_{n}^{j}(\lambda)$ is the $j$-th elementary symmetric function in the $\lambda_{j}$ 's. Thus, to prove the lemma, we only have to check that

$$
\sum_{0 \leqslant j \leqslant q}(-1)^{q-j} \sigma_{n}^{j}(\lambda)-\mathbb{1}_{\left\{\lambda_{q+1}<1\right\}}(-1)^{q} \prod_{1 \leqslant j \leqslant n}\left(1-\lambda_{j}\right) \geqslant 0 .
$$

This is easily done by induction on $n$ (just split apart the parameter $\lambda_{n}$ and write $\sigma_{n}^{j}(\lambda)=\sigma_{n-1}^{j}(\lambda)+$ $\left.\sigma_{n-1}^{j-1}(\lambda) \lambda_{n}\right)$.

We apply here Lemma 9.46 with

$$
\alpha=g_{k}^{\gamma}(z, x, u), \quad \beta=\beta_{k}=\frac{1}{k r}\left(1+\frac{1}{2}+\ldots+\frac{1}{k}\right)\left(\Theta_{A, h_{A}}+r \gamma\right),
$$

which are both semi-positive by our assumption. The analogue of (9.32) leads to

$$
\begin{aligned}
& \int_{X_{k}^{\mathrm{GG}}\left(L_{k}, \leqslant 1\right)} \Theta_{L_{k}, \Psi_{h, p, \varepsilon}^{*}+k r-1}^{*} \\
& =\frac{(n+k r-1) !}{n !(k !)^{r}(k r-1) !} \int_{z \in X} \int_{(x, u) \in \Delta_{k-1} \times\left(S^{2 r-1}\right)^{k}} \mathbb{1}_{g_{k}^{\gamma}-\beta_{k}, \leqslant 1}\left(g_{k}^{\gamma}-\beta_{k}\right)^{n} d \nu_{k, r}(x) d \mu(u) \\
& \geqslant \frac{(n+k r-1) !}{n !(k !)^{r}(k r-1) !} \int_{z \in X} \int_{(x, u) \in \Delta_{k-1} \times\left(S^{2 r-1}\right)^{k}}\left(\left(g_{k}^{\gamma}\right)^{n}-n\left(g_{k}^{\gamma}\right)^{n-1} \wedge \beta_{k}\right) d \nu_{k, r}(x) d \mu(u) .
\end{aligned}
$$

The resulting integral now produces a "closed formula" which can be expressed solely in terms of Chern classes (at least if we assume that $\gamma$ is the Chern form of some semi-positive line bundle). It is just a matter of routine to find a sufficient condition for the positivity of the integral. One can first observe that $g_{k}^{\gamma}$ is bounded from above by taking the trace of $\left(c_{i j \alpha \beta}\right)$, in this way we get

$$
0 \leqslant g_{k}^{\gamma} \leqslant\left(\sum_{1 \leqslant s \leqslant k} \frac{x_{s}}{s}\right)\left(\Theta_{\operatorname{det} V^{*}}+r \gamma\right)
$$

where the right hand side no longer depends on $u \in\left(S^{2 r-1}\right)^{k}$. Also, $g_{k}^{\gamma}$ can be written as a sum of semi-positive $(1,1)$-forms

$$
g_{k}^{\gamma}=\sum_{1 \leqslant s \leqslant k} \frac{x_{s}}{s} \theta^{\gamma}\left(u_{s}\right), \quad \theta^{\gamma}(u)=\sum_{i, j, \alpha, \beta} c_{i j \alpha \beta}^{\gamma} u_{\alpha} \bar{u}_{\beta} d z_{i} \wedge d \bar{z}_{j},
$$

and hence for $k \geqslant n$ we have

$$
\left(g_{k}^{\gamma}\right)^{n} \geqslant n ! \sum_{1 \leqslant s_{1}<\ldots<s_{n} \leqslant k} \frac{x_{s_{1}} \ldots x_{s_{n}}}{s_{1} \ldots s_{n}} \theta^{\gamma}\left(u_{s_{1}}\right) \wedge \theta^{\gamma}\left(u_{s_{2}}\right) \wedge \ldots \wedge \theta^{\gamma}\left(u_{s_{n}}\right) .
$$


Since $\int_{S^{2 r-1}} \theta^{\gamma}(u) d \mu(u)=\frac{1}{r} \operatorname{Tr}\left(\Theta_{V^{*}}+\gamma\right)=\frac{1}{r} \Theta_{\operatorname{det} V^{*}}+\gamma$, we infer from this

$$
\begin{aligned}
& \int_{(x, u) \in \Delta_{k-1} \times\left(S^{2 r-1}\right)^{k}}\left(g_{k}^{\gamma}\right)^{n} d \nu_{k, r}(x) d \mu(u) \\
& \geqslant n ! \sum_{1 \leqslant s_{1}<\ldots<s_{n} \leqslant k} \frac{1}{s_{1} \ldots s_{n}}\left(\int_{\Delta_{k-1}} x_{1} \ldots x_{n} d \nu_{k, r}(x)\right)\left(\frac{1}{r} \Theta_{\operatorname{det} V^{*}}+\gamma\right)^{n} .
\end{aligned}
$$

By putting everything together, we conclude:

9.47. Theorem. Assume that $\Theta_{V^{*}} \geqslant-\gamma \otimes \operatorname{Id}_{V^{*}}$ with a semi-positive $(1,1)$-form $\gamma$ on $X$. Then the Morse integral of the line bundle

$$
L_{k}=\mathcal{O}_{X_{k}^{\mathrm{GG}}}(1) \otimes \pi_{k}^{*} \mathcal{O}\left(-\frac{1}{k r}\left(1+\frac{1}{2}+\ldots+\frac{1}{k}\right) A\right), \quad A \geqslant 0
$$

satisfies for $k \geqslant n$ the inequality

$$
\begin{aligned}
& \frac{1}{(n+k r-1) !} \int_{X_{k}^{\mathrm{GG}}\left(L_{k}, \leqslant 1\right)} \Theta_{L_{k}, \Psi_{h, p, \varepsilon}^{*}+k r-1}^{*} \\
& \quad \geqslant \frac{1}{n !(k !)^{r}(k r-1) !} \int_{X} c_{n, r, k}\left(\Theta_{\operatorname{det} V^{*}}+r \gamma\right)^{n}-c_{n, r, k}^{\prime}\left(\Theta_{\operatorname{det} V^{*}}+r \gamma\right)^{n-1} \wedge\left(\Theta_{A, h_{A}}+r \gamma\right)
\end{aligned}
$$

where

$$
\begin{aligned}
c_{n, r, k} & =\frac{n !}{r^{n}}\left(\sum_{1 \leqslant s_{1}<\ldots<s_{n} \leqslant k} \frac{1}{s_{1} \ldots s_{n}}\right) \int_{\Delta_{k-1}} x_{1} \ldots x_{n} d \nu_{k, r}(x), \\
c_{n, r, k}^{\prime} & =\frac{n}{k r}\left(1+\frac{1}{2}+\ldots+\frac{1}{k}\right) \int_{\Delta_{k-1}}\left(\sum_{1 \leqslant s \leqslant k} \frac{x_{s}}{s}\right)^{n-1} d \nu_{k, r}(x) .
\end{aligned}
$$

Especially we have a lot of sections in $H^{0}\left(X_{k}^{\mathrm{GG}}, m L_{k}\right), m \gg 1$, as soon as the difference occurring in $(*)$ is positive.

The statement is also true for $k<n$, but then $c_{n, r, k}=0$ and the lower bound $(*)$ cannot be positive. By Corollary 9.11, it still provides a non-trivial lower bound for $h^{0}\left(X_{k}^{\mathrm{GG}}, m L_{k}\right)-$ $h^{1}\left(X_{k}^{\mathrm{GG}}, m L_{k}\right)$, though. For $k \geqslant n$ we have $c_{n, r, k}>0$ and $(*)$ will be positive if $\Theta_{\operatorname{det} V^{*}}$ is large enough. By Formula 9.20 we have

$$
c_{n, r, k}=\frac{n !(k r-1) !}{(n+k r-1) !} \sum_{1 \leqslant s_{1}<\ldots<s_{n} \leqslant k} \frac{1}{s_{1} \ldots s_{n}} \geqslant \frac{(k r-1) !}{(n+k r-1) !},
$$

(with equality for $k=n$ ). On the other hand, for any multi-index $\left(\beta_{1}, \ldots, \beta_{k}\right) \in \mathbb{N}^{k}$ with $\sum \beta_{s}=p$, the Hölder inequality implies

$$
\int_{\Delta_{k-1}} x_{1}^{\beta_{1}} \ldots x_{k}^{\beta_{k}} d \nu_{k, r}(x) \leqslant \prod_{s=1}\left(\int_{\Delta_{k-1}} x_{s}^{p} d \nu_{k, r}(x)\right)^{\beta_{s} / p}=\int_{\Delta_{k-1}} x_{1}^{p} d \nu_{k, r}(x) .
$$

An expansion of $\left(\sum_{1 \leqslant s \leqslant k} \frac{x_{s}}{s}\right)^{n-1}$ by means of the multinomial formula then yields

$$
\int_{\Delta_{k-1}}\left(\sum_{1 \leqslant s \leqslant k} \frac{x_{s}}{s}\right)^{n-1} d \nu_{k, r}(x) \leqslant \int_{\Delta_{k-1}}\left(\sum_{1 \leqslant s \leqslant k} \frac{1}{s}\right)^{n-1} x_{1}^{n-1} d \nu_{k, r}(x) .
$$

On the other hand, it is obvious that $\int_{\Delta_{k-1}}\left(\sum_{1 \leqslant s \leqslant k} \frac{x_{s}}{s}\right)^{n-1} d \nu_{k, r}(x) \geqslant \int_{\Delta_{k-1}} x_{1}^{n-1} d \nu_{k, r}(x)$, thus the error in the above upper bound is at most by a factor $\left(1+\frac{1}{2}+\ldots+\frac{1}{k}\right)^{n} \leqslant(1+\log k)^{n}$. From this, we infer again by Formula (9.20) that

$$
\begin{aligned}
c_{n, r, k}^{\prime} & \leqslant \frac{n}{k r}\left(1+\frac{1}{2}+\ldots+\frac{1}{k}\right)^{n} \int_{\Delta_{k-1}} x_{1}^{n-1} d \nu_{k, r}(x), \\
& =\frac{n}{k r}\left(1+\frac{1}{2}+\ldots+\frac{1}{k}\right)^{n} \frac{(n+r-2) !}{(r-1) !} \frac{(k r-1) !}{(n+k r-2) !} .
\end{aligned}
$$


Since $\frac{n+k r-1}{k}=r+\frac{n-1}{k} \leqslant n+r-1$, our bounds (9.48) and (9.49) imply

$$
\begin{aligned}
& \frac{c_{n, r, k}^{\prime}}{c_{n, r, k}} \leqslant \frac{n}{k}\left(1+\frac{1}{2}+\ldots+\frac{1}{k}\right)^{n} \frac{(n+r-2) !}{r !}(n+k r-1), \\
& \frac{c_{n, r, k}^{\prime}}{c_{n, r, k}} \leqslant n\left(1+\frac{1}{2}+\ldots+\frac{1}{k}\right)^{n} \frac{(n+r-1) !}{r !} .
\end{aligned}
$$

The right hand side of (9.51) increases with $r$. For $r \leqslant n$, the Stirling formula yields

$$
\frac{c_{n, r, k}^{\prime}}{c_{n, r, k}}<(1+\log k)^{n} \frac{(2 n) !}{2 n !}<(1+\log k)^{n} \frac{\sqrt{2 n}\left(\frac{2 n}{e}\right)^{2 n}}{2 \sqrt{n}\left(\frac{n}{e}\right)^{n}}=\frac{1}{\sqrt{2}}\left(4 e^{-1} n(1+\log k)\right)^{n} .
$$

Up to the constant $4 e^{-1}$, this is essentially the same bound as the one obtained in [Dem12], which, however, included a numerical mistake, making unclear whether the constant $4 e^{-1}>1$ could be dropped there, as would follow from the claimed estimate. We will later need the following particular values (cf. Formula (9.20) and [Dem11, Lemma 2.20]):

$$
\begin{array}{lll}
c_{2,2,2}=\frac{1}{20}, & c_{2,2,2}^{\prime}=\frac{9}{16}, & \frac{c_{2,2,2}^{\prime}}{c_{2,2,2}}=\frac{45}{4}, \\
c_{3,3,3}=\frac{1}{990}, & c_{3,3,3}^{\prime}=\frac{451}{4860}, & \frac{c_{3,3,3}^{\prime}}{c_{3,3,3}}=\frac{4961}{54} .
\end{array}
$$

\section{Hyperbolicity Properties of Hypersurfaces of High DEGREe}

\section{A. Global generation of the twisted tangent space of the universal family}

In [Siu02, Siu04], Y.T. Siu developed a new strategy to produce jet differentials, involving meromorphic vector fields on the total space of jet bundles - these vector fields are used to differentiate the sections of $E_{k, m}^{\mathrm{GG}}$ so as to produce new ones with less zeroes. The approach works especially well on universal families of hypersurfaces in projective space, thanks to the good positivity properties of the relative tangent bundle, as shown by L. Ein [Ein88, Ein91] and C. Voisin [Voi96]. This allows at least to prove the hyperbolicity of generic surfaces and generic 3-dimensional hypersurfaces of sufficiently high degree. We reproduce here the improved approach given by [Pau08] for the twisted global generation of the tangent space of the space of vertical two jets. The situation of $k$-jets in arbitrary dimension $n$ is substantially more involved, details can be found in [Mer09].

Consider the universal hypersurface $\mathcal{X} \subset \mathbb{P}^{n+1} \times \mathbb{P}^{N_{d}}$ of degree $d$ given by the equation

$$
\sum_{|\alpha|=d} A_{\alpha} Z^{\alpha}=0
$$

where $[Z] \in \mathbb{P}^{n+1},[A] \in \mathbb{P}^{N_{d}}, \alpha=\left(\alpha_{0}, \ldots, \alpha_{n+1}\right) \in \mathbb{N}^{n+2}$ and

$$
N_{d}=\left(\begin{array}{c}
n+d+1 \\
d
\end{array}\right)-1
$$

Finally, we denote by $\mathcal{V} \subset \mathcal{X}$ the vertical tangent space, i.e. the kernel of the projection

$$
\pi: \mathcal{X} \rightarrow U \subset \mathbb{P}^{N_{d}}
$$

where $U$ is the Zariski open set parametrizing smooth hypersurfaces, and by $J_{k} \mathcal{V}$ the bundle of $k$-jets of curves tangent to $\mathcal{V}$, i.e. curves contained in the fibers $X_{s}=\pi^{-1}(s)$. The goal is to describe certain meromorphic vector fields on the total space of $J_{k} \mathcal{V}$. By an explicit calculation of vector fields in coordinates, according to Siu's strategy, Păun [Pau08] was able to prove:

10.1. Theorem. The twisted tangent space $T_{J_{2}} \mathcal{V} \otimes \mathcal{O}_{\mathbb{P} 3}(7) \otimes \mathcal{O}_{\mathbb{P} N_{d}}(1)$ is generated over by its global sections over the complement $J_{2} \mathcal{V} \backslash \mathcal{W}$ of the Wronskian locus $\mathcal{W}$. Moreover, one can choose generating global sections that are invariant with respect to the action of $\mathbb{G}_{2}$ on $J_{2} \mathcal{V}$. 
By similar, but more computationally intensive arguments [Mer09], one can investigate the higher dimensional case. The following result strengthens the initial announcement of [Siu04].

10.2. Theorem. Let $J_{k}^{\mathrm{vert}}(\mathcal{X})$ be the space of vertical $k$-jets of the universal hypersurface

$$
\mathcal{X} \subset \mathbb{P}^{n+1} \times \mathbb{P}^{N_{d}}
$$

parametrizing all projective hypersurfaces $X \subset \mathbb{P}^{n+1}$ of degree $d$. Then for $k=n$, there exist constants $c_{n}$ and $c_{n}^{\prime}$ such that the twisted tangent bundle

$$
T_{J_{k}^{\text {vert }}(\mathcal{X})} \otimes \mathcal{O}_{\mathbb{P}^{n+1}}\left(c_{n}\right) \otimes \mathcal{O}_{\mathbb{P}^{N_{d}}}\left(c_{n}^{\prime}\right)
$$

is generated by its global $\mathbb{G}_{k}$-invariant sections outside a certain exceptional algebraic subset $\Sigma \subset$ $J_{k}^{\text {vert }}(\mathcal{X})$. One can take either $c_{n}=\frac{1}{2}\left(n^{2}+5 n\right), c_{n}^{\prime}=1$ and $\Sigma$ defined by the vanishing of certain Wronskians, or $c_{n}=n^{2}+2 n$ and a smaller set $\widetilde{\Sigma} \subset \Sigma$ defined by the vanishing of the 1-jet part.

\section{B. General strategy of Proof}

Let again $\mathcal{X} \subset \mathbb{P}^{n+1} \times \mathbb{P}^{N_{d}}$ be the universal hypersurface of degree $d$ in $\mathbb{P}^{n+1}$. (10.3) Assume that we can prove the existence of a non-zero polynomial differential operator

$$
P \in H^{0}\left(\mathcal{X}, E_{k, m}^{\mathrm{GG}} T_{\mathcal{X}}^{*} \otimes \mathcal{O}(-A)\right)
$$

where $A$ is an ample divisor on $\mathcal{X}$, at least over some Zariski open set $U$ in the base of the projection $\pi: \mathcal{X} \rightarrow U \subset \mathbb{P}^{N_{d}}$.

Observe that we now have a lot of techniques to do this; the existence of $P$ over the family follows from lower semi-continuity in the Zariski topology, once we know that such a section $P$ exists on a generic fiber $X_{s}=\pi^{-1}(s)$. Let $\mathcal{Y} \subset \mathcal{X}$ be the set of points $x \in \mathcal{X}$ where $P(x)=0$, as an element in the fiber of the vector bundle $\left.E_{k, m}^{\mathrm{GG}} T_{\mathcal{X}}^{*} \otimes \mathcal{O}(-A)\right)$ at $x$. Then $\mathcal{Y}$ is a proper algebraic subset of $\mathcal{X}$, and after shrinking $U$ we may assume that $Y_{s}=\mathcal{Y} \cap X_{s}$ is a proper algebraic subset of $X_{s}$ for every $s \in U$.

(10.4) Assume also, according to Theorems 10.1 and 10.2, that we have enough global holomorphic $\mathbb{G}_{k}$-invariant vector fields $\theta_{i}$ on $J_{k} \mathcal{V}$ with values in the pull-back of some ample divisor $B$ on $\mathcal{X}$, in such a way that they generate $T_{J_{k}} \mathcal{V} \otimes p_{k}^{*} B$ over the dense open set $\left(J_{k} \mathcal{V}\right)^{\mathrm{reg}}$ of regular $k$-jets, i.e. $k$-jets with non-zero first derivative (here $p_{k}: J_{k} \mathcal{V} \rightarrow \mathcal{X}$ is the natural projection).

Considering jet differentials $P$ as functions on $J_{k} \mathcal{V}$, the idea is to produce new ones by taking differentiations

$$
Q_{j}:=\theta_{j_{1}} \ldots \theta_{j_{\ell}} P, \quad 0 \leqslant \ell \leqslant m, j=\left(j_{1}, \ldots, j_{\ell}\right) .
$$

Since the $\theta_{j}$ 's are $\mathbb{G}_{k}$-invariant, they are in particular $\mathbb{C}^{*}$-invariant; thus

$$
Q_{j} \in H^{0}\left(\mathcal{X}, E_{k, m}^{\mathrm{GG}} T_{\mathcal{X}}^{*} \otimes \mathcal{O}(-A+\ell B)\right)
$$

(and $Q$ is in fact $\mathbb{G}_{k}^{\prime}$ invariant as soon as $P$ is). In order to be able to apply the vanishing theorems of $\S 8$, we need $(A-m B)$ to be ample, so $A$ has to be large compared to $B$. If $f: \mathbb{C} \rightarrow X_{s}$ is an entire curve contained in some fiber $X_{s} \subset \mathcal{X}$, its lifting $j_{k}(f): \mathbb{C} \rightarrow J_{k} \mathcal{V}$ has to lie in the zero divisors of all sections $Q_{j}$. However, every non-zero polynomial of degree $m$ has at any point some non-zero derivative of order $\ell \leqslant m$. Therefore, at any point where the $\theta_{i}$ generate the tangent space to $J_{k} \mathcal{V}$, we can find some non-vanishing section $Q_{j}$. By the assumptions on the $\theta_{i}$, the base locus of the $Q_{j}$ 's is contained in the union of $p_{k}^{-1}(\mathcal{Y}) \cup\left(J_{k} \mathcal{V}\right)^{\text {sing }}$; there is of course no way of getting a non-zero polynomial at points of $\mathcal{Y}$ where $P$ vanishes. Finally, we observe that $j_{k}(f)(\mathbb{C}) \not \subset\left(J_{k} \mathcal{V}\right)^{\text {sing }}$ (otherwise $f$ is constant). Therefore $j_{k}(f)(\mathbb{C}) \subset p_{k}^{-1}(\mathcal{Y})$ and thus $f(\mathbb{C}) \subset \mathcal{Y}$, i.e. $f(\mathbb{C}) \subset Y_{s}=\mathcal{Y} \cap X_{s}$.

10.5. Corollary. Let $\mathcal{X} \subset \mathbb{P}^{n+1} \times \mathbb{P}^{N_{d}}$ be the universal hypersurface of degree $d$ in $\mathbb{P}^{n+1}$. If $d \geqslant d_{n}$ is taken so large that conditions (10.3) and (10.4) are met with $(A-m B)$ ample, then the generic fiber $X_{s}$ of the universal family $\mathcal{X} \rightarrow U$ satisfies the Green-Griffiths conjecture, namely all entire curves $f: \mathbb{C} \rightarrow X_{s}$ are contained in a proper algebraic subvariety $Y_{s} \subset X_{s}$, and the $Y_{s}$ can be taken to form an algebraic subset $\mathcal{Y} \subset \mathcal{X}$. 
This is unfortunately not enough to get the hyperbolicity of $X_{s}$, because we would have to know that $Y_{s}$ itself is hyperbolic. However, one can use the following simple observation due to Diverio and Trapani [DT10]. The starting point is the following general, straightforward remark. Let $\mathcal{E} \rightarrow \mathcal{X}$ be a holomorphic vector bundle let $\sigma \in H^{0}(\mathcal{X}, \mathcal{E}) \neq 0$; then, up to factorizing by an effective divisor $D$ contained in the common zeroes of the components of $\sigma$, one can view $\sigma$ as a section

$$
\sigma \in H^{0}\left(\mathcal{X}, \mathcal{E} \otimes \mathcal{O}_{\mathcal{X}}(-D)\right)
$$

and this section now has a zero locus without divisorial components. Here, when $n \geqslant 2$, a very generic fiber $X_{s}$ has Picard number one by the Noether-Lefschetz theorem, and so, after shrinking $U$ if necessary, we can assume that $\mathcal{O}_{\mathcal{X}}(-D)$ is the restriction of $\mathcal{O}_{\mathbb{P}^{n+1}}(-p), p \geqslant 0$ by the effectivity of $D$. Hence $D$ can be assumed to be nef. After performing this simplification, $(A-m B)$ is replaced by $(A-m B+D)$, which is still ample if $(A-m B)$ is ample. As a consequence, we may assume $\operatorname{codim} \mathcal{Y} \geqslant 2$, and after shrinking $U$ again, that all $Y_{s}$ have $\operatorname{codim} Y_{s} \geqslant 2$.

10.6. Additional statement. In corollary 10.5, under the same hypotheses (10.3) and (10.4), one can take all fibers $Y_{s}$ to have codim $Y_{s} \geqslant 2$.

This is enough to conclude that $X_{s}$ is hyperbolic if $n=\operatorname{dim} X_{s} \leqslant 3$. In fact, this is clear if $n=2$ since the $Y_{s}$ are then reduced to points. If $n=3$, the $Y_{s}$ are at most curves, but we know by Ein and Voisin that a very generic hypersurface $X_{s} \subset \mathbb{P}^{4}$ of degree $d \geqslant 7$ does not possess any rational or elliptic curve. Hence $Y_{s}$ is hyperbolic and so is $X_{s}$, for $s$ generic.

10.7. Corollary. Assume that $n=2$ or $n=3$, and that $\mathcal{X} \subset \mathbb{P}^{n+1} \times \mathbb{P}^{N_{d}}$ is the universal hypersurface of degree $d \geqslant d_{n} \geqslant 2 n+1$ so large that conditions (10.3) and (10.4) are met with $(A-m B)$ ample. Then the very generic hypersurface $X_{s} \subset \mathbb{P}^{n+1}$ of degree $d$ is hyperbolic.

\section{C. Proof of the Green-Griffiths ConjeCture for Generic hypersurfaces in $\mathbb{P}^{n+1}$}

One of the first significant steps towards the Green-Griffiths conjecture is the result of Diverio, Merker and Rousseau [DMR10], confirming the statement when $X \subset \mathbb{P}_{\mathbb{C}}^{n+1}$ is a generic hypersurface of large degree $d$. Their proof yields a non-optimal lower bound $d \geqslant 2^{n^{5}}$ for the degree; it is based on an essential way on Siu's strategy as detailed in $\S 10 . \mathrm{B}$, combined with the earlier techniques of [Dem95]. Using our improved bounds from $\S 9 . D$, we obtain here a better estimate (actually, an estimate $O\left(\exp \left(n^{1+\varepsilon}\right)\right)$ of exponential order 1 rather than 5$)$. For the algebraic degeneracy of entire curves in open complements $X=\mathbb{P}^{n} \backslash H$, a better bound $d \geqslant 5 n^{2} n^{n}$ has been obtained by Darondeau [Dar14, Dar16b].

10.8. Theorem. A generic hypersurface $X \subset \mathbb{P}^{n+1}$ of degree $d \geqslant d_{n}$ with

$$
d_{2}=286, \quad d_{3}=7316, \quad d_{n}=\left\lfloor\frac{n^{4}}{\sqrt{2}}\left(4 e^{-1} n(1+\log n)\right)^{n}\right\rfloor \quad \text { for } n \geqslant 4,
$$

satisfies the Green-Griffiths conjecture.

Proof. Let us apply Theorem 9.47 with $V=T_{X}, r=n$ and $k=n$. The main starting point is the well known fact that $T_{\mathbb{P}^{n+1}}^{*} \otimes \mathcal{O}_{\mathbb{P}^{n+1}}(2)$ is semi-positive (in fact, generated by its sections). Hence the exact sequence

$$
0 \rightarrow \mathcal{O}_{\mathbb{P}^{n+1}}(-d) \rightarrow T_{\mathbb{P}^{n+1} \mid X}^{*} \rightarrow T_{X}^{*} \rightarrow 0
$$

implies that $T_{X}^{*} \otimes \mathcal{O}_{X}(2) \geqslant 0$. We can therefore take $\gamma=\Theta_{\mathcal{O}(2)}=2 \omega$ where $\omega$ is the FubiniStudy metric. Moreover $\operatorname{det}\left(V^{*}\right)=K_{X}=\mathcal{O}_{X}(d-n-2)$ has curvature $(d-n-2) \omega$, and thus $\Theta_{\operatorname{det}\left(V^{*}\right)}+r \gamma=(d+n-2) \omega$. The Morse integral to be computed when $A=\mathcal{O}_{X}(p)$ is

$$
\int_{X}\left(c_{n, n, n}(d+n-2)^{n}-c_{n, n, n}^{\prime}(d+n-2)^{n-1}(p+2 n)\right) \omega^{n}
$$


so the critical condition we need is

$$
d+n-2>\frac{c_{n, n, n}^{\prime}}{c_{n, n, n}}(p+2 n) .
$$

On the other hand, Siu's differentiation technique requires $\frac{m}{n^{2}}\left(1+\frac{1}{2}+\ldots+\frac{1}{n}\right) A-m B$ to be ample, where $B=\mathcal{O}_{X}\left(n^{2}+2 n\right)$ by Merker's result (Theorem 10.2). This ampleness condition yields

$$
\frac{1}{n^{2}}\left(1+\frac{1}{2}+\ldots+\frac{1}{n}\right) p-\left(n^{2}+2 n\right)>0,
$$

so one easily sees that it is enough to take $p=n^{4}-2 n$ for $n \geqslant 3$. Our estimates $\left(9.52_{n}\right)$ give the expected bound $d_{n}$.

Thanks to 10.6, one also obtains the generic hyperbolicity of 2 and 3-dimensional hypersurfaces of large degree.

10.9. Theorem. For $n=2$ or $n=3$, a generic hypersurface $X \subset \mathbb{P}^{n+1}$ of degree $d \geqslant d_{n}$ is Kobayashi hyperbolic.

By using more explicit calculations of Chern classes (and invariant jets rather than GreenGriffiths jets) Diverio-Trapani [DT10] obtained the better lower bound $d \geqslant d_{3}=593$ in dimension 3 . In the case of surfaces, Păun [Pau08] obtained $d \geqslant d_{2}=18$, using deep results of McQuillan [McQ98].

One may wonder whether it is possible to use jets of order $k<n$ in the proof of Theorems 10.8 and 10.9. Diverio [Div08] showed that the answer is negative (his proof is based on elementary facts of representation theory and a vanishing theorem of Brückmann-Rackwitz [BR90]):

10.10. Proposition ([Div08]). Let $X \subset \mathbb{P}^{n+1}$ be a smooth hypersurface. Then

$$
H^{0}\left(X, E_{k, m}^{\mathrm{GG}} T_{X}^{*}\right)=0
$$

for $m \geqslant 1$ and $1 \leqslant k<n$. More generally, if $X \subset \mathbb{P}^{n+s}$ is a smooth complete intersection of codimension $s$, there are no global jet differentials for $m \geqslant 1$ and $k<n / s$.

\section{Strong General type Condition and the GGL COnjecture}

\section{A. A partial Result towards the Green-Griffiths-Lang Conjecture}

The main result of this section is a proof of the partial solution to the Green-Griffiths-Lang conjecture asserted in Theorem 0.15. The following important "induction step" can be derived by Corollary 9.39. Here $D_{k}$ denotes again the sequence of "vertical divsors" defined in (6.9).

11.1. Proposition. Let $(X, V)$ be a directed pair where $X$ is projective algebraic. Take an irreducible algebraic subset $Z \not \subset D_{k}$ of the associated $k$-jet Semple bundle $X_{k}$ that projects onto $X_{k-1}$, $k \geqslant 1$, and assume that the induced directed space $(Z, W) \subset\left(X_{k}, V_{k}\right)$ is of general type modulo $X_{\bullet} \rightarrow X, \operatorname{rank} W \geqslant 1$. Then there exists a divisor $\Sigma \subset Z_{\ell}$ in a sufficiently high stage of the Semple tower $\left(Z_{\ell}, W_{\ell}\right)$ associated with $(Z, W)$, such that every non-constant holomorphic map $f: \mathbb{C} \rightarrow X$ whose $k$-jet defines a morphism $f_{[k]}:\left(\mathbb{C}, T_{\mathbb{C}}\right) \rightarrow(Z, W)$ also satisfies $f_{[k+\ell]}(\mathbb{C}) \subset \Sigma$.

Proof. Our hypothesis is that we can find an embedded resolution of singularities

$$
\mu_{\ell_{0}}:\left(\widehat{Z}_{\ell_{0}} \subset \widehat{X}_{k+\ell_{0}}\right) \rightarrow\left(Z_{\ell_{0}} \subset X_{k+\ell_{0}}\right), \quad \ell_{0} \geqslant 0
$$

and $p \in \mathbb{Q}_{\geqslant 0}$ such that

$$
K_{\widehat{W}_{\ell_{0}}}^{\bullet} \otimes \mathcal{O}_{\widehat{Z}_{\ell_{0}}}(p)_{\uparrow \widehat{Z}_{\ell_{0}}} \quad \text { is big over } \widehat{Z}_{\ell_{0}} .
$$

Since Corollary 9.39 and the related lower bound of $h^{0}$ are universal in the category of directed varieties, we can apply them by replacing $(X, V)$ with $\left(\widehat{Z}_{\ell_{0}}, \widehat{W}_{\ell_{0}}\right), r$ with $r_{0}=\operatorname{rank} W$, and $F$ by

$$
F_{\ell_{0}}=\mathcal{O}_{\widehat{Z}_{\ell_{0}}}(p) \otimes \mu_{\ell_{0}}^{*} \pi_{k+\ell_{0}, 0}^{*} \mathcal{O}_{X}(-\varepsilon A),
$$


where $A$ is an ample divisor on $X$ and $\varepsilon \in \mathbb{Q}_{>0}$. The assumptions show that $K_{\widehat{W}_{\ell_{0}}} \otimes F_{\ell_{0}}$ is still big on $\widehat{Z}_{\ell_{0}}$ for $\varepsilon$ small enough, therefore, by applying our theorem and taking $m \gg \ell \gg \ell_{0}$, we get a large number of (metric bounded) sections of

$$
\begin{aligned}
\mathcal{O}_{\widehat{Z}_{\ell}}(m) & \otimes \widehat{\pi}_{k+\ell, k+\ell_{0}}^{*} \mathcal{O}\left(\frac{m}{\ell r_{0}}\left(1+\frac{1}{2}+\ldots+\frac{1}{\ell}\right) F_{\ell_{0}}\right) \\
& =\mathcal{O}_{\widehat{Z}_{\ell}}(m a \bullet) \otimes \mu_{\ell}^{*} \pi_{k+\ell, 0}^{*} \mathcal{O}\left(-\frac{m \varepsilon}{\ell r_{0}}\left(1+\frac{1}{2}+\ldots+\frac{1}{\ell}\right) A\right)_{\mid \widehat{Z}_{\ell}} \\
& \subset \mathcal{O}_{\widehat{Z}_{\ell}}((1+\lambda) m) \otimes \mu_{\ell}^{*} \pi_{k+\ell, 0}^{*} \mathcal{O}\left(-\frac{m \varepsilon}{\ell r_{0}}\left(1+\frac{1}{2}+\ldots+\frac{1}{\ell}\right) A\right)_{\mid \widehat{Z}_{\ell}},
\end{aligned}
$$

where $\mu_{\ell}:\left(\widehat{Z}_{\ell} \subset \widehat{X}_{k+\ell}\right) \rightarrow\left(Z_{\ell} \subset X_{k+\ell}\right)$ is an embedded resolution dominating $\widehat{X}_{k+\ell_{0}}$, and $a_{\bullet} \in \mathbb{Q}_{+}^{\ell^{\prime}}$ a positive weight of the form $(0, \ldots, \lambda, \ldots, 0,1)$ with some non-zero component $\lambda \in \mathbb{Q}_{+}$at index $\ell_{0}$. Let $\widehat{\Sigma} \subset \widehat{Z}_{\ell}$ be the divisor of such a section. We apply the fundamental vanishing theorem 8.9 to lifted curves $\widehat{f}_{[k+\ell]}: \mathbb{C} \rightarrow \widehat{Z}_{\ell}$ and sections of (11.2), and conclude that $\widehat{f}_{[k+\ell}(\mathbb{C}) \subset \widehat{\Sigma}$. Therefore $f_{[k+\ell]}(\mathbb{C}) \subset \Sigma:=\mu_{\ell}(\widehat{\Sigma})$ and Proposition 11.1 is proved.

We now introduce the ad hoc condition that will enable us to check the GGL conjecture.

11.3. Definition. Let $(X, V)$ be a directed pair where $X$ is projective algebraic. We say that $(X, V)$ is "strongly of general type" if it is of general type and for every irreducible algebraic set $Z \subsetneq X_{k}$, $Z \not \subset D_{k}$, that projects onto $X$, the induced directed structure $(Z, W) \subset\left(X_{k}, V_{k}\right)$ is of general type modulo $X_{\bullet} \rightarrow X$.

11.4. Example. The situation of a product $(X, V)=\left(X^{\prime}, V^{\prime}\right) \times\left(X^{\prime \prime}, V^{\prime \prime}\right)$ described in $(0.14)$ shows that $(X, V)$ can be of general type without being strongly of general type. In fact, if $\left(X^{\prime}, V^{\prime}\right)$ and $\left(X^{\prime \prime}, V^{\prime \prime}\right)$ are of general type, then $K_{V}=\mathrm{pr}^{*} K_{V^{\prime}} \otimes \mathrm{pr}^{\prime \prime *} K_{V^{\prime \prime}}$ is big, so $(X, V)$ is again of general type. However

$$
Z=P\left(\mathrm{pr}^{\prime *} V^{\prime}\right)=X_{1}^{\prime} \times X^{\prime \prime} \subset X_{1}
$$

has a directed structure $W=\operatorname{pr}^{\prime *} V_{1}^{\prime}$ which does not possess a big canonical bundle over $Z$, since the restriction of $K_{W}$ to any fiber $\left\{x^{\prime}\right\} \times X^{\prime \prime}$ is trivial. The higher stages $\left(Z_{k}, W_{k}\right)$ of the Semple tower of $(Z, W)$ are given by $Z_{k}=X_{k+1}^{\prime} \times X^{\prime \prime}$ and $W_{k}=\operatorname{pr}^{*} V_{k+1}^{\prime}$, so it is easy to see that $\mathrm{GG}_{k}(X, V)$ contains $Z_{k-1}$. Since $Z_{k}$ projects onto $X$, we have here $\mathrm{GG}(X, V)=X$ (see [DR15] for more sophisticated indecomposable examples).

11.5. Hypersurface case. Assume that $Z \neq D_{k}$ is an irreducible hypersurface of $X_{k}$ that projects onto $X_{k-1}$. To simplify things further, also assume that $V$ is non-singular. Since the Semple jetbundles $X_{k}$ form a tower of $\mathbb{P}^{r-1}$-bundles, their Picard groups satisfy $\operatorname{Pic}\left(X_{k}\right) \simeq \operatorname{Pic}(X) \oplus \mathbb{Z}^{k}$ and we have $\mathcal{O}_{X_{k}}(Z) \simeq \mathcal{O}_{X_{k}}\left(a_{\bullet}\right) \otimes \pi_{k, 0}^{*} B$ for some $a_{\bullet} \in \mathbb{Z}^{k}$ and $B \in \operatorname{Pic}(X)$, where $a_{k}=d>0$ is the relative degree of the hypersurface over $X_{k-1}$. Let $\sigma \in H^{0}\left(X_{k}, \mathcal{O}_{X_{k}}(Z)\right)$ be the section defining $Z$ in $X_{k}$. The induced directed variety $(Z, W)$ has $\operatorname{rank} W=r-1=\operatorname{rank}(V)-1$ and Formula (7.25) yields $K_{V_{k}}=\mathcal{O}_{X_{k}}\left(-(r-1) 1_{\bullet}\right) \otimes \pi_{k, 0}^{*}\left(K_{V}\right)$. We claim that

$$
K_{W} \supset\left(K_{V_{k}} \otimes \mathcal{O}_{X_{k}}(Z)\right)_{\lceil Z} \otimes \mathcal{J}_{S}=\left(\mathcal{O}_{X_{k}}\left(a_{\bullet}-(r-1) 1_{\bullet}\right) \otimes \pi_{k, 0}^{*}\left(B \otimes K_{V}\right)\right)_{\lceil Z} \otimes \mathcal{J}_{S}
$$

where $S \subsetneq Z$ is the set (containing $Z_{\text {sing }}$ ) where $\sigma$ and $d \sigma_{\uparrow V_{k}}$ both vanish, and $\mathcal{J}_{S}$ is the ideal locally generated by the coefficients of $d \sigma_{\uparrow V_{k}}$ along $Z=\sigma^{-1}(0)$. In fact, the intersection $W=T_{Z} \cap V_{k}$ is transverse on $Z \backslash S$; then (11.5.1) can be seen by looking at the morphism

$$
V_{k \mid Z} \stackrel{d \sigma_{\mid V_{k}}}{\longrightarrow} \mathcal{O}_{X_{k}}(Z)_{\mid Z}
$$

and observing that the contraction by $K_{V_{k}}=\Lambda^{r} V_{k}^{*}$ provides a metric bounded section of the canonical sheaf $K_{W}$. In order to investigate the positivity properties of $K_{W}$, one has to show that $B$ cannot be too negative, and in addition to control the singularity set $S$. The second point is 
a priori very challenging, but we get useful information for the first point by observing that $\sigma$ provides a morphism $\pi_{k, 0}^{*} \mathcal{O}_{X}(-B) \rightarrow \mathcal{O}_{X_{k}}\left(a_{\bullet}\right)$, whence a non-trivial morphism

$$
\mathcal{O}_{X}(-B) \rightarrow E_{a \bullet}:=\left(\pi_{k, 0}\right)_{*} \mathcal{O}_{X_{k}}\left(a_{\bullet}\right)
$$

By [Dem95, Section 12], there exists a filtration on $E_{a}$ such that the graded pieces are irreducible representations of GL $(V)$ contained in $\left(V^{*}\right)^{\otimes \ell}, \ell \leqslant\left|a_{\bullet}\right|$. Therefore we get a non-trivial morphism

$$
\mathcal{O}_{X}(-B) \rightarrow\left(V^{*}\right)^{\otimes \ell}, \quad \ell \leqslant\left|a_{\bullet}\right| .
$$

If we know about certain (semi-)stability properties of $V$, this can be used to control the negativity of $B$.

We further need the following useful concept that slightly generalizes entire curve loci.

11.6. Definition. If $Z$ is an algebraic set contained in some stage $X_{k}$ of the Semple tower of $(X, V)$, we define its "induced entire curve locus" $\operatorname{IEL}_{X, V}(Z) \subset Z$ to be the Zariski closure of the union $\bigcup f_{[k]}(\mathbb{C})$ of all jets of entire curves $f:\left(\mathbb{C}, T_{\mathbb{C}}\right) \rightarrow(X, V)$ such that $f_{[k]}(\mathbb{C}) \subset Z$.

We have of course $\operatorname{IEL}_{X, V}\left(\operatorname{IEL}_{X, V}(Z)\right)=\operatorname{IEL}_{X, V}(Z)$ by definition. It is not hard to check that modulo certain "vertical divisors" of $X_{k}$, the $\operatorname{IEL}_{X, V}(Z)$ locus is essentially the same as the entire curve locus $\operatorname{ECL}(Z, W)$ of the induced directed variety, but we will not use this fact here. Notice that if $Z=\bigcup Z_{\alpha}$ is a decomposition of $Z$ into irreducible components, then

$$
\operatorname{IEL}_{X, V}(Z)=\bigcup_{\alpha} \operatorname{IEL}_{X, V}\left(Z_{\alpha}\right) .
$$

Since IEL $\mathrm{IL}_{X, V}\left(X_{k}\right)=\mathrm{ECL}_{k}(X, V)$, proving the Green-Griffiths-Lang property amounts to showing that $\operatorname{IEL}_{X, V}(X) \subsetneq X$ in the stage $k=0$ of the tower. The basic step of our approach is expressed in the following statement.

11.7. Proposition. Let $(X, V)$ be a directed variety and $p_{0} \leqslant n=\operatorname{dim} X, p_{0} \geqslant 1$. Assume that there is an integer $k_{0} \geqslant 0$ such that for every $k \geqslant k_{0}$ and every irreducible algebraic set $Z \subsetneq X_{k}$, $Z \not \subset D_{k}$, such that $\operatorname{dim} \pi_{k, k_{0}}(Z) \geqslant p_{0}$, the induced directed structure $(Z, W) \subset\left(X_{k}, V_{k}\right)$ is of general type modulo $X_{\bullet} \rightarrow X$. Then $\operatorname{dim} \mathrm{ECL}_{k_{0}}(X, V)<p_{0}$.

Proof. We argue here by contradiction, assuming that $\operatorname{dim} \mathrm{ECL}_{k_{0}}(X, V) \geqslant p_{0}$. If

$$
p_{0}^{\prime}:=\operatorname{dim} \operatorname{ECL}_{k_{0}}(X, V)>p_{0}
$$

and if we can prove the result for $p_{0}^{\prime}$, we will already get a contradiction. Hence we can assume without loss of generality that $\operatorname{dim} \mathrm{ECL}_{k_{0}}(X, V)=p_{0}$. The main argument consists of producing inductively an increasing sequence of integers

$$
k_{0}<k_{1}<\ldots<k_{j}<\ldots
$$

and directed varieties $\left(Z^{j}, W^{j}\right) \subset\left(X_{k_{j}}, V_{k_{j}}\right)$ satisfying the following properties :

(11.7.1) $Z^{0}$ is one of the irreducible components of $\operatorname{ECL}_{k_{0}}(X, V)$ and $\operatorname{dim} Z^{0}=p_{0}$;

(11.7.2) $Z^{j}$ is one of the irreducible components of $\operatorname{ECL}_{k_{j}}(X, V)$ and $\pi_{k_{j}, k_{0}}\left(Z^{j}\right)=Z^{0}$;

(11.7.3) for all $j \geqslant 0, \operatorname{IEL}_{X, V}\left(Z^{j}\right)=Z^{j}$ and $\operatorname{rank} W_{j} \geqslant 1$;

(11.7.4) for all $j \geqslant 0$, the directed variety $\left(Z^{j+1}, W^{j+1}\right)$ is contained in some stage (of order $\ell_{j}=$ $\left.k_{j+1}-k_{j}\right)$ of the Semple tower of $\left(Z^{j}, W^{j}\right)$, namely

$$
\left(Z^{j+1}, W^{j+1}\right) \subsetneq\left(Z_{\ell_{j}}^{j}, W_{\ell_{j}}^{j}\right) \subset\left(X_{k_{j+1}}, V_{k_{j+1}}\right)
$$

and

$$
W^{j+1}=\overline{T_{Z^{j+1}}, \cap W_{\ell_{j}}^{j}}=\overline{T_{Z^{j+1}}, \cap V_{k_{j}}}
$$

is the induced directed structure; moreover $\pi_{k_{j+1}, k_{j}}\left(Z^{j+1}\right)=Z^{j}$. 
(11.7.5) for all $j \geqslant 0$, we have $Z^{j+1} \subsetneq Z_{\ell_{j}}^{j}$ but $\pi_{k_{j+1}, k_{j+1}-1}\left(Z^{j+1}\right)=Z_{\ell_{j}-1}^{j}$.

For $j=0$, we simply take $Z^{0}$ to be one of the irreducible components $S_{\alpha}$ of $\mathrm{ECL}_{k_{0}}(X, V)$ such that $\operatorname{dim} S_{\alpha}=p_{0}$, which exists by our hypothesis that $\operatorname{dim} \mathrm{ECL}_{k_{0}}(X, V)=p_{0}$. Clearly, $\mathrm{ECL}_{k_{0}}(X, V)$ is the union of the $\operatorname{IEL}_{X, V}\left(S_{\alpha}\right)$ and we have $\operatorname{IEL}_{X, V}\left(S_{\alpha}\right)=S_{\alpha}$ for all those components. Thus $\operatorname{IEL}_{X, V}\left(Z^{0}\right)=Z^{0}$ and $\operatorname{dim} Z^{0}=p_{0}$. Assume that $\left(Z^{j}, W^{j}\right)$ has been constructed. The subvariety $Z^{j}$ cannot be contained in the vertical divisor $D_{k_{j}}$. In fact no irreducible algebraic set $Z$ such that $\operatorname{IEL}_{X, V}(Z)=Z$ can be contained in a vertical divisor $D_{k}$, because $\pi_{k, k-2}\left(D_{k}\right)$ corresponds to stationary jets in $X_{k-2}$; as every non-constant curve $f$ has non-stationary points, its $k$-jet $f_{[k]}$ cannot be entirely contained in $D_{k}$; also the induced directed structure $(Z, W)$ must satisfy rank $W \geqslant 1$, otherwise $\operatorname{IEL}_{X, V}(Z) \subsetneq Z$. Condition (11.7.2) implies that $\operatorname{dim} \pi_{k_{j}, k_{0}}\left(Z^{j}\right) \geqslant p_{0}$. Therefore $\left(Z^{j}, W^{j}\right)$ is of general type modulo $X_{\bullet} \rightarrow X$ by the assumptions of the proposition. Thanks to Proposition 2.5, we get an algebraic subset $\Sigma \subsetneq Z_{\ell}^{j}$ in some stage of the Semple tower $\left(Z_{\ell}^{j}\right)$ of $Z^{j}$ such that every entire curve $f:\left(\mathbb{C}, T_{\mathbb{C}}\right) \rightarrow(X, V)$ satisfying $f_{\left[k_{j}\right]}(\mathbb{C}) \subset Z^{j}$ also satisfies $f_{\left[k_{j}+\ell\right]}(\mathbb{C}) \subset \Sigma$. By definition, this implies the first inclusion in the sequence

$$
Z^{j}=\operatorname{IEL}_{X, V}\left(Z^{j}\right) \subset \pi_{k_{j}+\ell, k_{j}}\left(\operatorname{IEL}_{X, V}(\Sigma)\right) \subset \pi_{k_{j}+\ell, k_{j}}(\Sigma) \subset Z^{j}
$$

(the other ones being obvious), so we have in fact an equality throughout. Let $\left(S_{\alpha}^{\prime}\right)$ be the irreducible components of $\operatorname{IEL}_{X, V}(\Sigma)$. We have $\operatorname{IEL}_{X, V}\left(S_{\alpha}^{\prime}\right)=S_{\alpha}^{\prime}$ and one of the components $S_{\alpha}^{\prime}$ must satisfy

$$
\pi_{k_{j}+\ell, k_{j}}\left(S_{\alpha}^{\prime}\right)=Z^{j}=Z_{0}^{j} .
$$

We take $\ell_{j} \in[1, \ell]$ to be the smallest order such that $Z^{j+1}:=\pi_{k_{j}+\ell, k_{j}+\ell_{j}}\left(S_{\alpha}^{\prime}\right) \subsetneq Z_{\ell_{j}}^{j}$, and set $k_{j+1}=k_{j}+\ell_{j}>k_{j}$. By definition of $\ell_{j}$, we have $\pi_{k_{j+1}, k_{j+1}-1}\left(Z^{j+1}\right)=Z_{\ell_{j}-1}^{j}$, otherwise $\ell_{j}$ would not be minimal. We then get $\pi_{k_{j+1}, k_{j}}\left(Z^{j+1}\right)=Z^{j}$ and thus $\pi_{k_{j+1}, k_{0}}\left(Z^{j+1}\right)=Z^{0}$ by induction, and all properties $(11.7 .1-11.7 .5)$ follow easily. Now, by Observation 7.29, we have

$$
\operatorname{rank} W^{j}<\operatorname{rank} W^{j-1}<\ldots<\operatorname{rank} W^{1}<\operatorname{rank} W^{0}=\operatorname{rank} V .
$$

This is a contradiction because we cannot have such an infinite sequence. Proposition 11.7 is proved.

The special case $k_{0}=0, p_{0}=n$ of Proposition 11.7 yields the following consequence.

11.8. Partial solution to the generalized GGL conjecture. Let $(X, V)$ be a directed pair that is strongly of general type. Then the Green-Griffiths-Lang conjecture holds true for $(X, V)$, namely $\operatorname{ECL}(X, V) \subsetneq X$; in other words there exists a proper algebraic variety $Y \subsetneq X$ such that every nonconstant holomorphic curve $f: \mathbb{C} \rightarrow X$ tangent to $V$ satisfies $f(\mathbb{C}) \subset Y$.

11.9. Remark. The proof is not very constructive, but it is however theoretically effective. By this we mean that if $(X, V)$ is strongly of general type and is taken in a bounded family of directed varieties, i.e. $X$ is embedded in some projective space $\mathbb{P}^{N}$ with some bound $\delta$ on the degree, and $P(V)$ also has bounded degree $\leqslant \delta^{\prime}$ when viewed as a subvariety of $P\left(T_{\mathbb{P}^{N}}\right)$, then one could theoretically derive bounds $d_{Y}\left(n, \delta, \delta^{\prime}\right)$ for the degree of the locus $Y$. Also, there would exist bounds $k_{0}\left(n, \delta, \delta^{\prime}\right)$ for the orders $k$ and bounds $d_{k}\left(n, \delta, \delta^{\prime}\right)$ for the degrees of subvarieties $Z \subset X_{k}$ that have to be checked in the definition of a pair of strong general type. In fact, [Dem11] produces more or less explicit bounds for the order $k$ such that Corollary 9.39 holds true. The degree of the divisor $\Sigma$ is given by a section of a certain twisted line bundle $\mathcal{O}_{X_{k}}(m) \otimes \pi_{k, 0}^{*} \mathcal{O}_{X}(-A)$ that we know to be big by an application of holomorphic Morse inequalities - and the bounds for the degrees of $\left(X_{k}, V_{k}\right)$ then provide bounds for $m$.

11.10. Remark. The condition that $(X, V)$ is strongly of general type seems to be related to some sort of stability condition. We are unsure what is the most appropriate definition, but here is one that makes sense. Fix an ample divisor $A$ on $X$. For every irreducible subvariety $Z \subset X_{k}$ 
that projects onto $X_{k-1}$ for $k \geqslant 1$, and $Z=X=X_{0}$ for $k=0$, we define the slope $\mu_{A}(Z, W)$ of the corresponding directed variety $(Z, W)$ to be

$$
\mu_{A}(Z, W)=\frac{\inf \lambda}{\operatorname{rank} W}
$$

where $\lambda$ runs over all rational numbers such that there exists $\ell \geqslant 0$, a modification $\widehat{Z}_{\ell} \rightarrow Z_{\ell}$ and $p \in \mathbb{Q}_{+}$for which

$$
K_{\widehat{W}_{\ell}} \otimes\left(\mathcal{O}_{\widehat{Z}_{\ell}}(p) \otimes \pi_{k+\ell, 0}^{*} \mathcal{O}(\lambda A)\right)_{\uparrow \widehat{Z}_{\ell}} \text { is big on } \widehat{Z}_{\ell}
$$

(again, we assume here that $Z \not \subset D_{k}$ for $k \geqslant 2$ ). Notice that by definition $(Z, W)$ is of general type modulo $X_{\bullet} \rightarrow X$ if and only if $\mu_{A}(Z, W)<0$, and that $\mu_{A}(Z, W)=-\infty$ if $\mathcal{O}_{\widehat{Z}_{\ell}}(1)$ is big for some $\ell$. Also, the proof of Lemma 7.24 shows that for any $(Z, W)$ we have $\mu_{A}\left(Z_{\ell}, W_{\ell}\right)=\mu_{A}(Z, W)$ for all $\ell \geqslant 0$. We say that $(X, V)$ is $A$-jet-stable (resp. A-jet-semi-stable) if $\mu_{A}(Z, W)<\mu_{A}(X, V)$ (resp. $\left.\mu_{A}(Z, W) \leqslant \mu_{A}(X, V)\right)$ for all $Z \subsetneq X_{k}$ as above. It is then clear that if $(X, V)$ is of general type and $A$-jet-semi-stable, then it is strongly of general type in the sense of Definition 11.3. It would be useful to have a better understanding of this condition of stability (or any other one that would have better properties).

\section{B. Algebraic Jet-hyperbolicity implies Kobayashi hyperbolicity}

Let $(X, V)$ be a directed variety, where $X$ is an irreducible projective variety; the concept still makes sense when $X$ is singular, by embedding $(X, V)$ in a projective space $\left(\mathbb{P}^{N}, T_{\mathbb{P}^{N}}\right)$ and taking the linear space $V$ to be an irreducible algebraic subset of $T_{\mathbb{P} n}$ that is contained in $T_{X}$ at regular points of $X$.

11.11. Definition. Let $(X, V)$ be a directed variety. We say that $(X, V)$ is algebraically jethyperbolic if for every $k \geqslant 0$ and every irreducible algebraic subvariety $Z \subset X_{k}$ that is not contained in the union $\Delta_{k}$ of vertical divisors, the induced directed structure $(Z, W)$ either satisfies $W=0$, or is of general type modulo $X_{\bullet} \rightarrow X$, i.e. there exists $\ell \geqslant 0$ and $p \in \mathbb{Q} \geqslant 0$ such that $K_{\widehat{W}_{\ell}}^{\bullet} \otimes \mathcal{O}_{\widehat{Z}_{\ell}}(p)$ is big over $\widehat{Z}_{\ell}$, for some modification $\left(\widehat{Z}_{\ell}, \widehat{W}_{\ell}\right)$ of the $\ell$-stage of the Semple tower of $(Z, W)$.

Proposition 7.33 can be restated:

11.12. Proposition. If a projective directed variety $(X, V)$ is such that $\mathcal{O}_{X_{\ell}}\left(a_{\bullet}\right)$ is ample for some $\ell \geqslant 1$ and some weight $a_{\bullet} \in \mathbb{Q}_{>0}^{\ell}$, then $(X, V)$ is algebraically jet-hyperbolic.

In a similar vein, one would prove that if $\mathcal{O}_{X_{\ell}}\left(a_{\bullet}\right)$ is big and the "augmented base locus" $B=\operatorname{Bs}\left(\mathcal{O}_{X_{\ell}}\left(a_{\bullet}\right) \otimes \pi_{l, 0}^{*} A^{-1}\right)$ projects onto a proper subvariety $B^{\prime}=\pi_{\ell, 0}(B) \subsetneq X$, then $(X, V)$ is strongly of general type. In general, Proposition 11.7 gives the following:

11.13. Theorem. Let $(X, V)$ be an irreducible projective directed variety that is algebraically jethyperbolic in the sense of the above definition. Then $(X, V)$ is Brody (or Kobayashi) hyperbolic, i.e. $\operatorname{ECL}(X, V)=\emptyset$.

Proof. Here we apply Proposition 11.7 with $k_{0}=0$ and $p_{0}=1$. It is enough to deal with subvarieties $Z \subset X_{k}$ such that $\operatorname{dim} \pi_{k, 0}(Z) \geqslant 1$; otherwise $W=0$ and can reduce $Z$ to a smaller subvariety by $(2.2)$. Then we conclude that $\operatorname{dim} \operatorname{ECL}(X, V)<1$. All entire curves tangent to $V$ have to be constant, and we conclude in fact that $\operatorname{ECL}(X, V)=\emptyset$.

\section{Proof of the Kobayashi CONJECture on Generic hyperbolicity}

We give here a simple proof of the Kobayashi conjecture, combining ideas of Green-Griffiths [GrGr80], Nadel [Nad89], Masuda-Noguchi [MaNo96], Demailly [Dem95], Siu-Yeung [SiYe96a], Shiffman-Zaidenberg [ShZa02], Brotbek [Brot17], Ya Deng [Deng16], in chronological order. Related ideas had been used earlier in [Xie15], and then in [BrDa17], to establish Debarre's conjecture on the ampleness of the cotangent bundle of generic complete intersections of codimension at least equal to dimension. 


\section{A. General Wronskian operators}

This section follows closely the work of D. Brotbek [Brot17]. Let $U$ be an open set of a complex manifold $X, \operatorname{dim} X=n$, and $s_{0}, \ldots, s_{k} \in \mathcal{O}_{X}(U)$ be holomorphic functions. To these functions, we can associate a Wronskian operator of order $k$ defined by

$$
W_{k}\left(s_{0}, \ldots, s_{k}\right)(f)=\left|\begin{array}{cccc}
s_{0}(f) & s_{1}(f) & \ldots & s_{k}(f) \\
D\left(s_{0}(f)\right) & D\left(s_{1}(f)\right) & \ldots & D\left(s_{k}(f)\right) \\
\vdots & \vdots & & \vdots \\
D^{k}\left(s_{0}(f)\right) & D^{k}\left(s_{1}(f)\right) & \ldots & D^{k}\left(s_{k}(f)\right)
\end{array}\right|
$$

where $f:(\mathbb{C}, 0) \ni t \mapsto f(t) \in U \subset X$ is a germ of holomorphic curve (or a $k$-jet of curve), and $D=\frac{d}{d t}$. For a biholomorphic change of variable $\varphi:(\mathbb{C}, 0) \rightarrow(\mathbb{C}, 0)$, we find by induction on $\ell$ a polynomial differential operator $p_{\ell, i}$ of order $\leqslant \ell$ acting on $\varphi$ satisfying

$$
D^{\ell}\left(s_{j}(f \circ \varphi)\right)=\varphi^{\prime \ell} D^{\ell}\left(s_{j}(f)\right) \circ \varphi+\sum_{i<\ell} p_{\ell, i}(\varphi) D^{i}\left(s_{j}(f)\right) \circ \varphi .
$$

It follows easily from this that

$$
W_{k}\left(s_{0}, \ldots, s_{k}\right)(f \circ \varphi)=\left(\varphi^{\prime}\right)^{1+2+\cdots+k} W_{k}\left(s_{0}, \ldots, s_{k}\right)(f) \circ \varphi,
$$

and hence $W_{k}\left(s_{0}, \ldots, s_{k}\right)(f)$ is an invariant differential operator of degree $k^{\prime}=\frac{1}{2} k(k+1)$. Especially, we get in this way a section that we denote somewhat sloppily

$$
W_{k}\left(s_{0}, \ldots, s_{k}\right)=\left|\begin{array}{cccc}
s_{0} & s_{1} & \ldots & s_{k} \\
D\left(s_{0}\right) & D\left(s_{1}\right) & \ldots & D\left(s_{k}\right) \\
\vdots & \vdots & & \vdots \\
D^{k}\left(s_{0}\right) & D^{k}\left(s_{1}\right) & \ldots & D^{k}\left(s_{k}\right)
\end{array}\right| \in H^{0}\left(U, E_{k, k^{\prime}} T_{X}^{*}\right) .
$$

12.3. Proposition. These Wronskian operators satisfy the following properties.

(a) $W_{k}\left(s_{0}, \ldots, s_{k}\right)$ is $\mathbb{C}$-multilinear and alternate in $\left(s_{0}, \ldots, s_{k}\right)$.

(b) For any $g \in \mathcal{O}_{X}(U)$, we have

$$
W_{k}\left(g s_{0}, \ldots, g s_{k}\right)=g^{k+1} W_{k}\left(s_{0}, \ldots, s_{k}\right) .
$$

Property 12.3 (b) is an easy consequence of the Leibniz formula

$$
D^{\ell}\left(g(f) s_{j}(f)\right)=\sum_{k=0}^{\ell}\left(\begin{array}{l}
\ell \\
k
\end{array}\right) D^{k}(g(f)) D^{\ell-k}\left(s_{j}(f)\right),
$$

by performing linear combinations of rows in the determinants. This property implies in its turn that for any $(k+1)$-tuple of sections $s_{0}, \ldots, s_{k} \in H^{0}(U, L)$ of a holomorphic line bundle $L \rightarrow X$, one can define more generally an operator

$$
W_{k}\left(s_{0}, \ldots, s_{k}\right) \in H^{0}\left(U, E_{k, k^{\prime}} T_{X}^{*} \otimes L^{k+1}\right) .
$$

In fact, when we compute the Wronskian in a local trivialization of $L_{\uparrow U}$, Property 12.3 (b) shows that the determinant is independent of the trivialization. Moreover, if $g \in H^{0}(U, G)$ for some line bundle $G \rightarrow X$, we have

$$
W_{k}\left(g s_{0}, \ldots, g s_{k}\right)=g^{k+1} W_{k}\left(s_{0}, \ldots, s_{k}\right) \in H^{0}\left(U, E_{k, k^{\prime}} T_{X}^{*} \otimes L^{k+1} \otimes G^{k+1}\right) .
$$

We consider here a line bundle $L \rightarrow X$ possessing a linear system $\Sigma \subset H^{0}(X, L)$ of global sections such that $W_{k}\left(s_{0}, \ldots, s_{k}\right) \not \equiv 0$ for generic elements $s_{0}, \ldots, s_{k} \in \Sigma$. We can then view $W_{k}\left(s_{0}, \ldots, s_{k}\right)$ as a section of $H^{0}\left(X_{k}, \mathcal{O}_{X_{k}}\left(k^{\prime}\right) \otimes \pi_{k, 0}^{*} L^{k+1}\right)$ on the $k$-stage $X_{k}$ of the Semple tower. Very roughly, the idea for the proof of the Kobayashi conjecture is to produce many such Wronskians, and to apply the fundamental vanishing theorem 8.15 to exclude the existence of entire curves. However, 
the vanishing theorem only holds for jet differentials in $H^{0}\left(X_{k}, \mathcal{O}_{X_{k}}\left(k^{\prime}\right) \otimes \pi_{k, 0}^{*} A^{-1}\right)$ with $A>0$, while the existence of sufficiently many sections $s_{j} \in H^{0}(X, L)$ can be achieved only when $L$ is ample, so the strategy seems a priori unapplicable. It turns out that one can sometimes arrange the Wronkian operator coefficients to be divisible by a section $\sigma_{\Delta} \in H^{0}\left(X, \mathcal{O}_{X}(\Delta)\right)$ possessing a large zero divisor $\Delta$, so that

$$
\sigma_{\Delta}^{-1} W_{k}\left(s_{0}, \ldots, s_{k}\right) \in H^{0}\left(X_{k}, \mathcal{O}_{X_{k}}\left(k^{\prime}\right) \otimes \pi_{k, 0}^{*}\left(L^{k+1} \otimes \mathcal{O}_{X}(-\Delta)\right)\right),
$$

and we can then hope that $\left.L^{k+1} \otimes \mathcal{O}_{X}(-\Delta)\right)<0$. Our goal is thus to find a variety $X$ and linear systems $\Sigma \subset H^{0}(X, L)$ for which the associated Wronskians $W_{k}\left(s_{0}, \ldots, s_{k}\right)$ have a very high divisibility. The study of the base locus of line bundles $\mathcal{O}_{X_{k}}\left(k^{\prime}\right) \otimes \pi_{k, 0}^{*}\left(L^{k+1} \otimes \mathcal{O}_{X}(-\Delta)\right)$ and their related positivity properties will be taken care of by using suitable blow-ups.

\section{B. Using a BLOW-UP of the Wronskian IDEAL SHEAF}

We consider again a linear system $\Sigma \subset H^{0}(X, L)$ producing some non-zero Wronskian sections $W_{k}\left(s_{0}, \ldots, s_{k}\right)$, so that $\operatorname{dim} \Sigma \geqslant k+1$. As the Wronskian is alternate and multilinear in the arguments $s_{j}$, we get a meromorphic map $X_{k}--\rightarrow P\left(\Lambda^{k+1} \Sigma^{*}\right)$ by sending a $k$-jet $\gamma=f_{[k]}(0) \in X_{k}$ to the point of projective coordinates $\left[W_{k}\left(u_{i_{0}}, \ldots, u_{i_{k}}\right)(f)(0)\right]_{i_{0}, \ldots, i_{k}}$ where $\left(u_{j}\right)_{j \in J}$ is a basis of $\Sigma$ and $i_{0}, \ldots, i_{k} \in J$ are in increasing order. This assignment factorizes through the Plücker embedding into a meromorphic map

$$
\Phi: X_{k} \rightarrow \operatorname{Gr}_{k+1}(\Sigma)
$$

into the Grassmannian of dimension $(k+1)$ subspaces of $\Sigma^{*}$ (or codimension $(k+1)$ subspaces of $\Sigma$, alternatively). In fact, if $L_{\uparrow U} \simeq U \times \mathbb{C}$ is a trivialization of $L$ in a neighborhood of a point $x_{0}=f(0) \in X$, we can consider the map $\Psi_{U}: X_{k} \rightarrow \operatorname{Hom}\left(\Sigma, \mathbb{C}^{k+1}\right)$ given by

$$
\pi_{k, 0}^{-1}(U) \ni f_{[k]} \mapsto\left(s \mapsto\left(D^{\ell}(s(f))_{0 \leqslant \ell \leqslant k}\right)\right),
$$

and associate either the kernel $\Xi \subset \Sigma$ of $\Psi_{U}\left(f_{[k]}\right)$, seen as a point $\Xi \in \operatorname{Gr}_{k+1}(\Sigma)$, or $\Lambda^{k+1} \Xi^{\perp} \subset$ $\Lambda^{k+1} \Sigma^{*}$, seen as a point of $P\left(\Lambda^{k+1} \Sigma^{*}\right)$ (assuming that we are at a point where the rank is equal to $(k+1)$ ). Let $\mathcal{O}_{\mathrm{Gr}}(1)$ be the tautological very ample line bundle on $\operatorname{Gr}_{k+1}(\Sigma)$ (equal to the restriction of $\left.\mathcal{O}_{P\left(\Lambda^{k+1} \Sigma^{*}\right)}(1)\right)$. By construction, $\Phi$ is induced by the linear system of sections

$$
W_{k}\left(u_{i_{0}}, \ldots, u_{i_{k}}\right) \in H^{0}\left(X_{k}, \mathcal{O}_{X_{k}}\left(k^{\prime}\right) \otimes \pi_{k, 0}^{*} L^{k+1}\right),
$$

and we thus get a natural isomorphism

$$
\mathcal{O}_{X_{k}}\left(k^{\prime}\right) \otimes \pi_{k, 0}^{*} L^{k+1} \simeq \Phi^{*} \mathcal{O}_{\mathrm{Gr}}(1) \quad \text { on } X_{k} \backslash B_{k},
$$

where $B_{k} \subset X_{k}$ is the base locus of our linear system of Wronskians. The presence of the indeterminacy set $B_{k}$ may create trouble in analyzing the positivity of our line bundles, so we are going to use an appropriate blow-up to resolve the indeterminacies. For this purpose, we introduce the ideal sheaf $\mathcal{J}_{k, \Sigma} \subset \mathcal{O}_{X_{k}}$ generated by the linear system $\Sigma$, and take a modification $\mu_{k, \Sigma}: \widehat{X}_{k, \Sigma} \rightarrow X_{k}$ in such a way that $\mu_{k, \Sigma}^{*} \mathcal{J}_{k, \Sigma}=\mathcal{O}_{\widehat{X}_{k, \Sigma}}\left(-F_{k, \Sigma}\right)$ for some divisor $F_{k, \Sigma}$ in $\widehat{X}_{k, \Sigma}$. Then $\Phi$ is resolved into a morphism $\Phi \circ \mu_{k, \Sigma}: \widehat{X}_{k, \Sigma} \rightarrow \operatorname{Gr}_{k+1}(\Sigma)$, and on $\widehat{X}_{k, \Sigma}$, (12.6) becomes an everywhere defined isomorphism

$$
\mu_{k, \Sigma}^{*}\left(\mathcal{O}_{X_{k}}\left(k^{\prime}\right) \otimes \pi_{k, 0}^{*} L^{k+1}\right) \otimes \mathcal{O}_{\widehat{X}_{k, \Sigma}}\left(-F_{k, \Sigma}\right) \simeq\left(\Phi \circ \mu_{k, \Sigma}\right)^{*} \mathcal{O}_{\mathrm{Gr}}(1)
$$

In fact, we can simply take $\widehat{X}_{k}$ to be the normalized blow-up of $\mathcal{J}_{k, \Sigma}$, i.e. the normalization of the closure $\Gamma \subset X_{k} \times \operatorname{Gr}_{k+1}(\Sigma)$ of the graph of $\Phi$ and $\mu_{k, \Sigma}: \widehat{X}_{k} \rightarrow X_{k}$ to be the composition of the normalization map $\widehat{X}_{k} \rightarrow \Gamma$ with the first projection $\Gamma \rightarrow X_{k}$. [The Hironaka desingularization theorem would possibly allow us to replace $\widehat{X}_{k}$ by a nonsingular modification, and $F_{k, \Sigma}$ by a simple normal crossing divisor on the desingularization; we will avoid doing so here, as we would otherwise need to show the existence of universal desingularizations when $\left(X_{t}, \Sigma_{t}\right)$ is a family of linear systems 
of $k$-jets of sections associated with a family of algebraic varieties]. The following basic lemma was observed by Ya Deng [Deng16].

12.8. Lemma. Locally over coordinate open sets $U \subset X$ on which $L_{\uparrow U}$ is trivial, there is a maximal "Wronskian ideal sheaf" $\mathcal{J}_{k}^{X} \supset \mathcal{J}_{k, \Sigma}$ in $\mathcal{O}_{X_{k}}$ achieved by linear systems $\Sigma \subset H^{0}(U, L)$. It is attained globally on $X$ whenever the linear system $\Sigma \subset H^{0}(X, L)$ generates $k$-jets of sections of $L$ at every point. Finally, it is "universal" in the sense that is does not depend on $L$ and behaves functorially under immersions: if $\psi: X \rightarrow Y$ is an immersion and $\mathcal{J}_{k}^{X}, \mathcal{J}_{k}^{Y}$ are the corresponding Wronskian ideal sheaves in $\mathcal{O}_{X_{k}}, \mathcal{O}_{Y_{k}}$, then $\psi_{k}^{*} \mathcal{J}_{k}^{Y}=\mathcal{J}_{k}^{X}$ with respect to the induced immersion $\psi_{k}: X_{k} \rightarrow Y_{k}$.

Proof. The (local) existence of such a maximal ideal sheaf is merely a consequence of the strong Noetherian property of coherent ideals. As observed at the end of section 6.A, the bundle $X_{k} \rightarrow X$ is a locally trivial tower of $\mathbb{P}^{n-1}$-bundles, with a fiber $\mathcal{R}_{n, k}$ that is a rational $k(n-1)$-dimensional variety; over any coordinate open set $U \subset X$ equipped with local coordinates $\left(z_{1}, \ldots, z_{n}\right) \in B(0, r) \subset \mathbb{C}^{n}$, it is isomorphic to the product $U \times \mathcal{R}_{n, k}$, the fiber over a point $x_{0} \in U$ being identified with the central fiber through a translation $(t \mapsto f(t)) \mapsto\left(t \mapsto x_{0}+f(t)\right)$ of germs of curves. In this setting, $\mathcal{J}_{k}^{X}$ is generated by the functions in $\mathcal{O}_{X_{k}}$ associated with Wronskians

$$
X_{k \uparrow U} \ni \xi=f_{[k]} \mapsto W_{k}\left(s_{0}, \ldots, s_{k}\right)(f) \in \mathcal{O}_{X_{k}}\left(k^{\prime}\right)_{\uparrow \mathcal{R}_{n, k}}, \quad s_{j} \in H^{0}\left(U, \mathcal{O}_{X}\right),
$$

by taking local trivializations $\mathcal{O}_{X_{k}}\left(k^{\prime}\right)_{\xi_{0}} \simeq \mathcal{O}_{X_{k}, \xi_{0}}$ at points $\xi_{0} \in X_{k}$. In fact, it is enough to take Wronskians associated with polynomials $s_{j} \in \mathbb{C}\left[z_{1}, \ldots, z_{n}\right]$. To see this, one can e.g. invoke Krull's lemma for local rings, which implies $\mathcal{J}_{k, \xi_{0}}^{X}=\bigcap_{\ell \geqslant 0}\left(\mathcal{J}_{k, \xi_{0}}^{X}+\mathfrak{m}_{\xi_{0}}^{\ell+1}\right)$, and to observe that $\ell$-jets of Wronskians $W_{k}\left(s_{0}, \ldots, s_{k}\right)\left(\bmod \mathfrak{m}_{\xi_{0}}^{\ell+1}\right)$ depend only on the $(k+\ell)$-jets of the sections $s_{j}$ in $\mathcal{O}_{X, x_{0}} / \mathfrak{m}_{x_{0}}^{k+\ell+1}$, where $x_{0}=\pi_{k, 0}\left(\xi_{0}\right)$. Therefore, polynomial sections $s_{j}$ or arbitrary holomorphic functions $s_{j}$ define the same $\ell$-jets of Wronskians for any $\ell$. Now, in the case of polynomials, it is clear that translations $(t \mapsto f(t)) \mapsto\left(t \mapsto x_{0}+f(t)\right)$ leave $\mathcal{J}_{k}^{X}$ invariant, hence $\mathcal{J}_{k}^{X}$ is the pull-back by the second projection $X_{k \mid U} \simeq U \times \mathcal{R}_{n, k} \rightarrow \mathcal{R}_{n, k}$ of its restriction to any of the fibers $\pi_{k, 0}^{-1}\left(x_{0}\right) \simeq \mathcal{R}_{n, k}$. As the $k$-jets of the $s_{j}$ 's at $x_{0}$ are sufficient to determine the restriction of our Wronskians to $\pi_{k, 0}^{-1}\left(x_{0}\right)$, the first two claims of Lemma 5.3 follow. The universality property comes from the fact that $L_{\uparrow U}$ is trivial (cf. $(12.3 \mathrm{~b})$ ) and that germs of sections of $\mathcal{O}_{X}$ extend to germs of sections of $\mathcal{O}_{Y}$ via the immersion $\psi$. (Notice that in this discussion, one may have to pick Taylor expansions of order $>k$ for $f$ to reach all points of the fiber $\pi_{k, 0}^{-1}\left(x_{0}\right)$, the order $2 k-1$ being sufficient by [Dem95, Prop. 5.11], but this fact does not play any role here). A consequence of universality is that $\mathcal{J}_{k}^{X}$ does not depend on coordinates nor on the geometry of $X$.

The above discussion combined with Lemma 12.8 leads to the following statement.

12.9. Proposition. Assume that $L$ generates all $k$-jets of sections (e.g. take $L=A^{p}$ with $A$ very ample and $p \geqslant k)$, and let $\Sigma \subset H^{0}(X, L)$ be a linear system that also generates $k$-jets of sections at any point of $X$. Then we have a universal isomorphism

$$
\mu_{k}^{*}\left(\mathcal{O}_{X_{k}}\left(k^{\prime}\right) \otimes \pi_{k, 0}^{*} L^{k+1}\right) \otimes \mathcal{O}_{\widehat{X}_{k, \Sigma}}\left(-F_{k}\right) \simeq\left(\Phi \circ \mu_{k}\right)^{*} \mathcal{O}_{\mathrm{Gr}_{k+1}(\Sigma)}(1),
$$

where $\mu_{k}: \widehat{X}_{k} \rightarrow X_{k}$ is the normalized blow-up of the (maximal) ideal sheaf $\mathcal{J}_{k}^{X} \subset \mathcal{O}_{X_{k}}$ associated with order $k$ Wronskians, and $F_{k}$ the universal divisor of $\widehat{X}_{k}$ resolving $\mathcal{J}_{k}^{X}$.

\section{C. Specialization to suitable hypersurfaces}

Let $Z$ be a non-singular $(n+1)$-dimensional projective variety, and let $A$ be a very ample divisor on $Z$; the fundamental example is of course $Z=\mathbb{P}^{n+1}$ and $A=\mathcal{O}_{\mathbb{P}^{n+1}}$ (1). Our goal is to show that a sufficiently general ( $n$-dimensional) hypersurface $X=\{x \in Z ; \sigma(x)=0\}$ defined by a section $\sigma \in H^{0}\left(Z, A^{d}\right), d \gg 1$, is Kobayashi hyperbolic. A basic idea, inspired by some of the main past contributions, such as Brody-Green [BrGr77], Nadel [Nad89], Masuda-Noguchi [MaNo96], Shiffman-Zaidenberg [ShZa02] and [Xie15], is to consider hypersurfaces defined by special equations, 
e.g. deformations of unions of hyperplane sections $\tau_{1} \cdots \tau_{d}=0$ or of Fermat-Waring hypersurfaces $\sum_{0 \leqslant j \leqslant N} \tau_{j}^{d}=0$, for suitable sections $\tau_{j} \in H^{0}(Z, A)$. Brotbek's main idea developed in [Brot17] is that a carefully selected hypersurface may have enough Wronskian sections to imply the ampleness of some tautological jet line bundle - a Zariski open property. Here, we take $\sigma \in H^{0}\left(X, A^{d}\right)$ equal to a sum of terms

$$
\sigma=\sum_{0 \leqslant j \leqslant N} a_{j} m_{j}^{\delta}, \quad a_{j} \in H^{0}\left(Z, A^{\rho}\right), m_{j} \in H^{0}\left(Z, A^{b}\right), n<N \leqslant k, d=\delta b+\rho,
$$

where $\delta \gg 1$ and the $m_{j}$ are "monomials" of the same degree $b$, i.e. products of $b$ "linear" sections $\tau_{I} \in H^{0}(Z, A)$, and the factors $a_{j}$ are general enough. The integer $\rho$ is taken in the range $[k, k+b-1]$, first to ensure that $H^{0}\left(Z, A^{\rho}\right)$ generates $k$-jets of sections, and second, to allow $d$ to be an arbitrary large integer (once $\delta \geqslant \delta_{0}$ has been chosen large enough).

The monomials $m_{j}$ will be chosen in such a way that for suitable $c \in \mathbb{N}, 1 \leqslant c \leqslant N$, any subfamily of $c$ terms $m_{j}$ shares one common factor $\tau_{I} \in H^{0}(X, A)$. To this end, we consider all subsets $I \subset\{0,1, \ldots, N\}$ with card $I=c$; there are $B:=\left(\begin{array}{c}N+1 \\ c\end{array}\right)$ subsets of this type. For all such $I$, we select sections $\tau_{I} \in H^{0}(Z, A)$ such that $\prod_{I} \tau_{I}=0$ is a simple normal crossing divisor in $Z$ (with all of its components of multiplicity 1 ). For $j=0,1, \ldots, N$ given, the number of subsets $I$ containing $j$ is $b:=\left(\begin{array}{c}N \\ c-1\end{array}\right)$. We put

$$
m_{j}=\prod_{I \ni j} \tau_{I} \in H^{0}\left(Z, A^{b}\right) .
$$

By construction, every family $m_{i_{1}}, \ldots, m_{i_{c}}$ of sections shares the common factor $\tau_{I} \in H^{0}(X, A)$ where $I=\left\{i_{1}, \ldots, i_{c}\right\}$. The first step consists in checking that we can achieve $X$ to be smooth with these constraints.

12.12. Lemma. Assume $N \geqslant c(n+1)$. Then, for a generic choice of the sections $a_{j} \in H^{0}\left(Z, A^{\rho}\right)$ and $\tau_{I} \in H^{0}(Z, A)$, the hypersurface $X=\sigma^{-1}(0) \subset Z$ defined by (12.10-12.11) is non-singular. Moreover, under the same condition for $N$, the intersection of $\prod \tau_{I}=0$ with $X$ can be taken to be a simple normal crossing divisor in $X$.

Proof. As the properties considered in the Lemma are Zariski open properties in terms of the $(N+B+1)$-tuple $\left(a_{j}, \tau_{I}\right)$, it is sufficient to prove the result for a specific choice of the $a_{j}$ 's: we fix here $a_{j}=\tilde{\tau}_{j} \tau_{I(j)}^{\rho-1}$ where $\tilde{\tau}_{j} \in H^{0}(X, A), 0 \leqslant j \leqslant N$ are new sections such that $\prod \tilde{\tau}_{j} \prod \tau_{I}=0$ is a simple normal crossing divisor, and $I(j)$ is any subset of cardinal $c$ containing $j$. Let $H$ be the hypersurface of degree $d$ of $\mathbb{P}^{N+B}$ defined in homogeneous coordinates $\left(z_{j}, z_{I}\right) \in \mathbb{C}^{N+B+1}$ by $h(z)=0$ where

$$
h(z)=\sum_{0 \leqslant j \leqslant N} z_{j} z_{I(j)}^{\rho-1} \prod_{I \ni j} z_{I}^{\delta},
$$

and consider the morphism $\Phi: Z \rightarrow \mathbb{P}^{N+B}$ such that $\Phi(x)=\left(\tilde{\tau}_{j}(x), \tau_{I}(x)\right)$. With our choice of the $a_{j}$ 's, we have $\sigma=h \circ \Phi$. Now, when the $\tilde{\tau}_{j}$ and $\tau_{I}$ are general enough, the map $\Phi$ defines an embedding of $Z$ into $\mathbb{P}^{N+B}$ (for this, one needs $N+B \geqslant 2(\operatorname{dim} Z)+1=2 n+3$, which is the case by our assumptions). Then, by definition, $X$ is isomorphic to the intersection of $H$ with $\Phi(Z)$. Changing generically the $\tilde{\tau}_{j}$ and $\tau_{I}$ 's can be achieved by composing $\Phi$ with a generic automorphism $g \in \operatorname{Aut}\left(\mathbb{P}^{N+B}\right)=\mathrm{PGL}_{N+B+1}(\mathbb{C})\left(\right.$ as $\mathrm{GL}_{N+B+1}(\mathbb{C})$ acts transitively on $(N+B+1)$-tuples of linearly independent linear forms). As $\operatorname{dim} g \circ \Phi(Z)=\operatorname{dim} Z=n+1$, Lemma 12.12 will follow from a standard Bertini argument if we can check that $\operatorname{Sing}(H)$ has codimension at least $(n+2)$ in $\mathbb{P}^{N+B}$. In fact, this condition implies $\operatorname{Sing}(H) \cap(g \circ \Phi(Z))=\emptyset$ for $g$ generic, while $g \circ \Phi(Z)$ can be chosen transverse to $\operatorname{Reg}(H)$. Now, a sufficient condition for smoothness is that one of the differentials $d z_{j}$, $0 \leqslant j \leqslant N$, appears with a non-zero factor in $d h(z)$ (just neglect the other differentials $* d z_{I}$ in this argument). We infer from this and the fact that $\delta \geqslant 2$ that $\operatorname{Sing}(H)$ consists of the locus defined by $\prod_{I \ni j} z_{I}=0$ for all $j=0,1, \ldots, N$. It is the union of the linear subspaces $z_{I_{0}}=\ldots=z_{I_{N}}=0$ for all possible choices of subsets $I_{j}$ such that $I_{j} \ni j$. Since card $I_{j}=c$, the equality $\bigcup I_{j}=\{0,1, \ldots, N\}$ 
implies that there are at least $\lceil(N+1) / c\rceil$ distinct subsets $I_{j}$ involved in each of these linear subspaces, and the equality can be reached. Therefore codim $\operatorname{Sing}(H)=\lceil(N+1) / c\rceil \geqslant n+2$ as soon as $N \geqslant c(n+1)$. By the same argument, we can assume that the intersection of $Z$ with at least $(n+2)$ distinct hyperplanes $z_{I}=0$ is empty. In order that $\prod \tau_{I}=0$ defines a normal crossing divisor at a point $x \in X$, it is sufficient to ensure that for any family $\mathcal{G}$ of coordinate hyperplanes $z_{I}=0, I \in \mathcal{G}$, with card $\mathcal{G} \leqslant n+1$, we have a "free" index $j \notin \bigcup_{I \in \mathcal{G}} I$ such that $x_{I} \neq 0$ for all $I \ni j$, so that $d h$ involves a non-zero term $* d z_{j}$ independent of the $d z_{I}, I \in \mathcal{G}$. If this fails, there must be at least $(n+2)$ hyperplanes $z_{I}=0$ containing $x$, associated either with $I \in \mathcal{G}$, or with other $I$ 's covering $\complement\left(\bigcup_{I \in \mathcal{G}} I\right)$. The corresponding bad locus is of codimension at least $(n+2)$ in $\mathbb{P}^{N+B}$ and can be avoided by $g(\Phi(Z))$ for a generic choice of $g \in \operatorname{Aut}\left(\mathbb{P}^{N+B}\right)$. Then $X \cap \bigcap_{I \in \mathcal{G}} \tau_{I}^{-1}(0)$ is smooth of codimension equal to card $\mathcal{G}$.

\section{D. Construction of highly Divisible Wronskians}

To any families $s, \hat{\tau}$ of sections $s_{1}, \ldots, s_{r} \in H^{0}\left(Z, A^{k}\right), \hat{\tau}_{1}, \ldots, \hat{\tau}_{r} \in H^{0}(Z, A)$, and to each subset $J \subset\{0,1, \ldots, N\}$ with card $J=c$, we associate a Wronskian operator of order $k$ (i.e. a $(k+1) \times(k+1)$-determinant $)$

$$
W_{k, s, \hat{\tau}, a, J}=W_{k}\left(s_{1} \hat{\tau}_{1}^{d-k}, \ldots, s_{r} \hat{\tau}_{r}^{d-k},\left(a_{j} m_{j}^{\delta}\right)_{j \in \mathrm{C} J}\right), \quad r=k+c-N,|\complement J|=N-c .
$$

We assume here again that the $\hat{\tau}_{j}$ are chosen so that $\prod \hat{\tau}_{j} \prod \tau_{I}=0$ defines a simple normal crossing divisor in $Z$ and $X$. Since $s_{j} \hat{\tau}_{j}^{d-k}, a_{j} m_{j}^{\delta} \in H^{0}\left(Z, A^{d}\right)$, formula (12.4) applied with $L=A^{d}$ implies that

$$
W_{k, s, \hat{\tau}, a, J} \in H^{0}\left(Z, E_{k, k^{\prime}} T_{Z}^{*} \otimes A^{(k+1) d}\right) .
$$

However, we are going to see that $W_{k, s, \hat{\tau}, a, J}$ and its restriction $W_{k, s, \hat{\tau}, a, J \mid X}$ are divisible by monomials $\hat{\tau}^{\alpha} \tau^{\beta}$ of very large degree, where $\hat{\tau}$, resp. $\tau$, denotes the collection of sections $\hat{\tau}_{j}$, resp. $\tau_{I}$ in $H^{0}(Z, A)$. In this way, we will see that we can even obtain a negative exponent of $A$ after simplifying $\hat{\tau}^{\alpha} \tau^{\beta}$ in $W_{k, s, \hat{\tau}, a, J\lceil X}$. This simplification process is a generalization of techniques already considered by [Siu87] and [Nad89] (and later [DeEG97]) in relation with the use of meromorphic connections of low pole order.

12.15. Lemma. Assume that $\delta \geqslant k$. Then the Wronskian operator $W_{k, s, \hat{\tau}, a, J}$, resp. $W_{k, s, \hat{\tau}, a, J\lceil X}$, is divisible by a monomial $\hat{\tau}^{\alpha} \tau^{\beta}$, resp. $\hat{\tau}^{\alpha} \tau^{\beta} \tau_{J}^{\delta-k}$ (with a multiindex notation $\hat{\tau}^{\alpha} \tau^{\beta}=\prod \hat{\tau}_{j}^{\alpha_{j}} \prod \tau_{I}^{\beta_{I}}$ ), and

$$
\alpha, \beta \geqslant 0, \quad|\alpha|=r(d-2 k), \quad|\beta|=(N+1-c)(\delta-k) b .
$$

Proof. $W_{k, s, \hat{\tau}, a, J}$ is obtained as a determinant whose $r$ first columns are the derivatives $D^{\ell}\left(s_{j} \hat{\tau}_{j}^{d-k}\right)$ and the last $(N+1-c)$ columns are the $D^{\ell}\left(a_{j} m_{j}^{\delta}\right)$, divisible respectively by $\hat{\tau}_{j}^{d-2 k}$ and $m_{j}^{\delta-k}$. As $m_{j}$ is of the form $\tau^{\gamma},|\gamma|=b$, this implies the divisibility of $W_{k, s, \hat{\tau}, a, J}$ by a monomial of the form $\hat{\tau}^{\alpha} \tau^{\beta}$, as asserted. Now, we explain why one can gain the additional factor $\tau_{J}^{\delta-k}$ dividing the

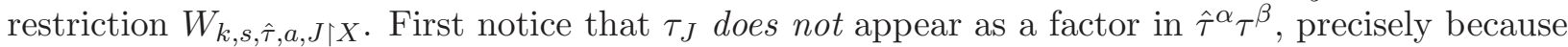
the Wronskian involves only terms $a_{j} m_{j}^{\delta}$ with $j \notin J$, and thus these $m_{j}$ 's do not contain $\tau_{J}$. Let us pick $j_{0}=\min (\complement J) \in\{0,1, \ldots, N\}$. Since $X$ is defined by $\sum_{0 \leqslant j \leqslant N} a_{j} m_{j}^{\delta}=0$, we have identically

$$
a_{j_{0}} m_{j_{0}}^{\delta}=-\sum_{i \in J} a_{i} m_{i}^{\delta}-\sum_{i \in \mathrm{C} J \backslash\left\{j_{0}\right\}} a_{i} m_{i}^{\delta}
$$

in restriction to $X$, whence (by the alternate property of $W_{k}(\bullet)$ )

$$
W_{k, s, \hat{\tau}, a, J\lceil X}=-\sum_{i \in J} W_{k}\left(s_{1} \hat{\tau}_{1}^{d-k}, \ldots, s_{r} \hat{\tau}_{r}^{d-k}, a_{i} m_{i}^{\delta},\left(a_{j} m_{j}^{\delta}\right)_{j \in \complement J \backslash\left\{j_{0}\right\}}\right)_{\lceil X} .
$$


However, all terms $m_{i}, i \in J$, contain by definition the factor $\tau_{J}$, and the derivatives $D^{\ell}(\bullet)$ leave us a factor $m_{i}^{\delta-k}$ at least. Therefore, the above restricted Wronskian is also divisible by $\tau_{J}^{\delta-k}$, thanks to the fact that $\prod \hat{\tau}_{j} \prod \tau_{I}=0$ forms a simple normal crossing divisor in $X$.

12.16. Corollary. For $\delta \geqslant k$, there exists a monomial $\hat{\tau}^{\alpha_{J}} \tau^{\beta_{J}}$ dividing $W_{k, s, \hat{\tau}, a, J \mid X}$ such that

$$
\left|\alpha_{J}\right|+\left|\beta_{J}\right|=(k+c-N)(d-2 k)+(N+1-c)(\delta-k) b+(\delta-k),
$$

and we have

$$
\widetilde{W}_{k, s, \hat{\tau}, a, J \mid X}:=\left(\hat{\tau}^{\alpha_{J}} \tau^{\beta_{J}}\right)^{-1} W_{k, s, \hat{\tau}, a, J\lceil X} \in H^{0}\left(X, E_{k, k^{\prime}} T_{X}^{*} \otimes A^{-p}\right)
$$

where

$$
p=\left|\alpha_{J}\right|+\left|\beta_{J}\right|-(k+1) d=(\delta-k)-(k+c-N) 2 k-(N+1+c)(k b+\rho) .
$$

In particular, we have $p>0$ for $\delta$ large enough (all other parameters being fixed or bounded), and under this assumption, the fundamental vanishing theorem 8.15 implies that all entire curves $f: \mathbb{C} \rightarrow X$ are annihilated by these Wronskian operators.

Proof. In fact,

$$
(k+1) d=(k+c-N) d+(N+1-c) d=(k+c-N) d+(N+1-c)(\delta b+\rho),
$$

and we get (12.17) by subtraction.

\section{E. Control of the base locus for sufficiently general COefFicients $a_{j}$ IN $\sigma$}

The next step is to control more precisely the base locus of these Wronskians and to find conditions on $N, k, c, d=b \delta+\rho$ ensuring that the base locus is empty for a generic choice of the sections $a_{j}$ in $\sigma=\sum a_{j} m_{j}$. Although we will not formally use it, the next lemma is useful to realize that the base locus is related to a natural rank condition.

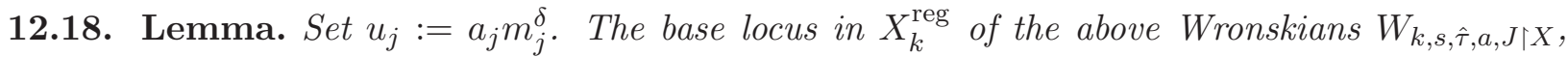
when $s, \hat{\tau}$ vary, consists of jets $f_{[k]}(0) \in X_{k}^{\mathrm{reg}}$ such that the matrix $\left(D^{\ell}\left(u_{j} \circ f\right)(0)\right)_{0 \leqslant \ell \leqslant k, j \in \mathrm{C} J}$ is not of maximal rank (i.e., of rank $<\operatorname{card} \complement J=N+1-c)$; if $\delta>k$, the base locus includes all jets $f_{[k]}(0)$ such that $f(0) \in \bigcup_{I \neq J} \tau_{I}^{-1}(0)$. When $J$ also varies, the base locus of all $W_{k, s, \hat{\tau}, a, J \mid X}$ in the Zariski open set $X_{k}^{\prime}:=X_{k}^{\mathrm{reg}} \backslash \bigcup_{|I|=c} \tau_{I}^{-1}(0)$ consists of all $k$-jets such that $\operatorname{rank}\left(D^{\ell}\left(u_{j} \circ f\right)(0)\right)_{0 \leqslant \ell \leqslant k, 0 \leqslant j \leqslant N} \leqslant$ $N-c$.

Proof. If $\delta>k$ and $m_{j} \circ f(0)=0$ for some $j \in J$, we have in fact $D^{\ell}\left(u_{j} \circ f\right)(0)=0$ for all derivatives $\ell \leqslant k$, because the exponents involved in all factors of the differentiated monomial $a_{j} m_{j}^{\delta}$ are at least equal to $\delta-k>0$. Hence the rank of the matrix cannot be maximal. Now, assume that $m_{j} \circ f(0) \neq 0$ for all $j \in \complement J$, i.e.

$$
x_{0}:=f(0) \in X \backslash \bigcup_{j \in \complement J} m_{j}^{-1}(0)=X \backslash \bigcup_{I \neq J} \tau_{I}^{-1}(0) .
$$

We take sections $\hat{\tau}_{j}$ so that $\hat{\tau}_{j}\left(x_{0}\right) \neq 0$, and then adjust the $k$-jet of the sections $s_{1}, \ldots, s_{r}$ in order to generate any matrix of derivatives $\left(D^{\ell}\left(s_{j}(f) \hat{\tau}_{j}(f)^{d-k}\right)(0)\right)_{0 \leqslant \ell \leqslant k, j \in \mathrm{C} J}$ (the fact that $f^{\prime}(0) \neq 0$ is used for this!). Therefore, by expanding the determinant according to the last $(N+1-c)$ columns, we see that the base locus is defined by the equations

$$
\operatorname{det}\left(D^{\ell}\left(u_{j}(f)\right)(0)\right)_{\ell \in L, j \in \complement J}=0, \quad \forall L \subset\{0,1, \ldots, k\},|L|=N+1-c,
$$

equivalent to the non-maximality of the rank. The last assertion follows by a simple linear algebra argument.

For a finer control of the base locus, we adjust the family of coefficients

$$
a=\left(a_{j}\right)_{0 \leqslant j \leqslant N} \in S:=H^{0}\left(Z, A^{\rho}\right)^{\oplus(N+1)}
$$


in our section $\sigma=\sum a_{j} m_{j}^{\delta} \in H^{0}\left(Z, A^{d}\right)$, and denote by $X_{a}=\sigma^{-1}(0) \subset Z$ the corresponding hypersurface. By Lemma 12.12, we know that there is a Zariski open set $U \subset S$ such that $X_{a}$ is smooth and $\prod \tau_{I}=0$ is a simple normal crossing divisor in $X_{a}$ for all $a \in U$. We consider the Semple tower $X_{a, k}:=\left(X_{a}\right)_{k}$ of $X_{a}$, the "universal blow-up" $\mu_{a, k}: \widehat{X}_{a, k} \rightarrow X_{a, k}$ of the Wronskian ideal sheaf $\mathcal{J}_{a, k}$ such that $\mu_{a, k}^{*} \mathcal{J}_{a, k}=\mathcal{O}_{\widehat{X}_{a, k}}\left(-F_{a, k}\right)$ for some "Wronskian divisor" $F_{a, k}$ in $\widehat{X}_{a, k}$. By the universality of this construction (cf. Lemma 12.8), we can also embed $X_{a, k}$ in the Semple tower $Z_{k}$ of $Z$, blow up the Wronskian ideal sheaf $\mathcal{J}_{k}^{Z}$ of $Z_{k}$ to get a Wronskian divisor $F_{k}^{Z}$ in $\widehat{Z}_{k}$ where $\mu_{k}: \widehat{Z}_{k} \rightarrow Z_{k}$ is the blow-up map. Then $F_{a, k}$ is the restriction of $F_{k}^{Z}$ to $\widehat{X}_{a, k} \subset \widehat{Z}_{k}$. Our section $\widetilde{W}_{k, s, \hat{\tau}, a, J\left\lceil X_{a}\right.}$ is the restriction of a meromorphic section defined on $Z$, namely

$$
\left(\hat{\tau}^{\alpha_{J}} \tau^{\beta_{J}}\right)^{-1} W_{k, s, \hat{\tau}, a, J}=\left(\hat{\tau}^{\alpha_{J}} \tau^{\beta_{J}}\right)^{-1} W_{k}\left(s_{1} \hat{\tau}_{1}^{d-k}, \ldots, s_{r} \hat{\tau}_{r}^{d-k},\left(a_{j} m_{j}^{\delta}\right)_{j \in \complement J}\right) .
$$

It induces over the Zariski open set $Z^{\prime}=Z \backslash \bigcup_{I} \tau_{I}^{-1}(0)$ a holomorphic section

$$
\sigma_{k, s, \hat{\tau}, a, J} \in H^{0}\left(\widehat{Z}_{k}^{\prime}, \mu_{k}^{*}\left(\mathcal{O}_{Z_{k}}\left(k^{\prime}\right) \otimes \pi_{k, 0}^{*} A^{-p}\right) \otimes \mathcal{O}_{\widehat{Z}_{k}}\left(-F_{k}^{Z}\right)\right)
$$

(notice that the relevant factors $\hat{\tau}_{j}$ remain divisible on the whole variety $Z$ ). By construction, thanks to the divisibility property explained in Lemma 12.15, the restriction of this section to $\widehat{X}_{a, k}^{\prime}=\widehat{X}_{a, k} \cap \widehat{Z}_{k}^{\prime}$ extends holomorphically to $\widehat{X}_{a, k}$, i.e.

$$
\sigma_{k, s, \hat{\tau}, a, J \mid \widehat{X}_{a, k}} \in H^{0}\left(\widehat{X}_{a, k}, \mu_{a, k}^{*}\left(\mathcal{O}_{X_{a, k}}\left(k^{\prime}\right) \otimes \pi_{k, 0}^{*} A^{-p}\right) \otimes \mathcal{O}_{\widehat{X}_{a, k}}\left(-F_{a, k}\right)\right) .
$$

(Here the fact that we took $\widehat{X}_{k, a}$ to be normal implies that the divided section is indeed holomorphic on $\widehat{X}_{k, a}$, as $\widehat{X}_{k, a} \cap \mu_{k}^{-1}\left(\pi_{k, 0}^{-1} \bigcap_{I \in \mathcal{G}} \tau_{I}^{-1}(0)\right)$ has the expected codimension $=$ card $\mathcal{G}$ for any family $\left.\mathcal{G}\right)$.

12.25. Lemma. Let $V$ be a finite dimensional vector space over $\mathbb{C}, \Psi: V^{p} \rightarrow \mathbb{C}$ a non-zero alternating multilinear form, and let $m, c \in \mathbb{N}, c<m \leqslant p, r=p+c-m \geqslant 0$. Then the subset $T \subset V^{m}$ of vectors $\left(v_{1}, \ldots, v_{m}\right) \in V^{m}$ such that

$$
\Psi\left(h_{1}, \ldots, h_{r},\left(v_{j}\right)_{j \in \complement}\right)=0 \quad \text { for all } J \subset\{1, \ldots, m\},|J|=c \text {, and all } h_{1}, \ldots, h_{r} \in V,
$$

is a closed algebraic subset of codimension $\geqslant(c+1)(r+1)$.

Proof. A typical example is $\Psi=\operatorname{det}$ on a $p$-dimensional vector space $V$, then $T$ consists of $m$-tuples of vectors of rank $<p-r$, and the assertion concerning the codimension is well known (we will reprove it anyway). In general, the algebraicity of $T$ is obvious. We argue by induction on $p$, the result being trivial for $p=1$ (the kernel of a non-zero linear form is indeed of codimension $\geqslant 1$ ). If $K$ is the kernel of $\Psi$, i.e. the subspace of vectors $v \in V$ such that $\Psi\left(h_{1}, \ldots, h_{p-1}, v\right)=0$ for all $h_{j} \in V$, then $\Psi$ induces an alternating multilinear form $\bar{\Psi}$ on $V / K$, whose kernel is equal to $\{0\}$. The proof is thus reduced to the case when $\operatorname{Ker} \Psi=\{0\}$. Notice that we must have $\operatorname{dim} V \geqslant p$, otherwise $\Psi$ would vanish. If card $\complement J=m-c=1$, condition $(*)$ implies that $v_{j} \in \operatorname{Ker} \Psi=\{0\}$ for all $j$, and hence $\operatorname{codim} T=\operatorname{dim} V^{m} \geqslant m p=(c+1)(r+1)$, as desired. Now, assume $m-c \geqslant 2$, fix $v_{m} \in V \backslash\{0\}$ and consider the non-zero alternating multilinear form on $V^{p-1}$ such that

$$
\Psi_{v_{m}}^{\prime}\left(w_{1}, \ldots, w_{p-1}\right):=\Psi\left(w_{1}, \ldots, w_{p-1}, v_{m}\right) .
$$

If $\left(v_{1}, \ldots, v_{m}\right) \in T$, then $\left(v_{1}, \ldots, v_{m-1}\right)$ belongs to the set $T_{v_{m}}^{\prime}$ associated with the new data $\left(\Psi_{v_{m}}^{\prime}, p-1, m-1, c, r\right)$. The induction hypothesis implies that $\operatorname{codim} T_{v_{m}}^{\prime} \geqslant(c+1)(r+1)$, and since the projection $T \rightarrow V$ to the first factor admits the $T_{v_{m}}^{\prime}$ as its fibers, we conclude that

$$
\operatorname{codim} T \cap\left((V \backslash\{0\}) \times V^{m-1}\right) \geqslant(c+1)(r+1) .
$$

By permuting the arguments $v_{j}$, we also conclude that

$$
\operatorname{codim} T \cap\left(V^{k-1} \times(V \backslash\{0\}) \times V^{m-k}\right) \geqslant(c+1)(r+1)
$$

for all $k=1, \ldots, m$. The union $\bigcup_{k}\left(V^{k-1} \times(V \backslash\{0\}) \times V^{m-k}\right) \subset V^{m}$ leaves out only $\{0\} \subset V^{m}$ whose codimension is at least $m p \geqslant(c+1)(r+1)$, so Lemma 12.25 follows. 
12.26. Proposition. Consider in $U \times \widehat{Z}_{k}^{\prime}$ the set $\Gamma$ of pairs $(a, \xi)$ such that $\sigma_{k, s, \hat{\tau}, a, J}(\xi)=0$ for all choices of $s, \hat{\tau}$ and $J \subset\{0,1, \ldots, N\}$ with card $J=c$. Then $\Gamma$ is an algebraic set of dimension

$$
\operatorname{dim} \Gamma \leqslant \operatorname{dim} S-(c+1)(k+c-N+1)+n+1+k n .
$$

As a consequence, if $(c+1)(k+c-N+1)>n+1+k n$, there exists $a \in U \subset S$ such that the base locus of the family of sections $\sigma_{k, s, \hat{\tau}, a, J}$ in $\widehat{X}_{a, k}$ lies over $\bigcup_{I} X_{a} \cap \tau_{I}^{-1}(0)$.

Proof. The idea is similar to [Brot17, Lemma 3.8], but somewhat simpler in the present context. Let us consider a point $\xi \in \widehat{Z}_{k}^{\prime}$ and the $k$-jet $f_{[k]}=\mu_{k}(\xi) \in Z_{k}^{\prime}$, so that $x=f(0) \in Z^{\prime}=Z \backslash \bigcup_{I} \tau_{I}^{-1}(0)$. Let us take the $\hat{\tau}_{j}$ such that $\hat{\tau}_{j}(x) \neq 0$. Then, we do not have to pay attention to the non-vanishing factors $\hat{\tau}^{\alpha_{J}} \tau^{\beta_{J}}$, and the $k$-jets of sections $m_{j}$ and $\hat{\tau}_{j}^{d-k}$ are invertible near $x$. Let $e_{A}$ be a local generator of $A$ near $x$ and $e_{\mathcal{L}}$ a local generator of the invertible sheaf

$$
\mathcal{L}=\mu_{k}^{*} \mathcal{O}_{Z_{k}}\left(k^{\prime}\right) \otimes \mathcal{O}_{\widehat{Z}_{k}}\left(-F_{k}^{Z}\right)
$$

near $\xi \in \widehat{Z}_{k}^{\prime}$. Let $J^{k} \mathcal{O}_{Z, x}=\mathcal{O}_{Z, x} / \mathfrak{m}_{Z, x}^{k+1}$ be the vector space of $k$-jets of functions on $Z$ at $x$. By definition of the Wronskian ideal and of the associated divisor $F_{k}^{Z}$, we have a non-zero alternating multilinear form

$$
\Psi:\left(J^{k} \mathcal{O}_{Z, x}\right)^{k+1} \rightarrow \mathbb{C}, \quad\left(g_{0}, \ldots, g_{k}\right) \mapsto \mu_{k}^{*} W_{k}\left(g_{0}, \ldots, g_{k}\right)(\xi) / e_{\mathcal{L}}(\xi) .
$$

The simultaneous vanishing of our sections at $\xi$ is equivalent to the vanishing of

$$
\Psi\left(s_{1} \hat{\tau}_{1}^{d-k} e_{A}^{-d}, \ldots, s_{r} \hat{\tau}_{r}^{d-k} e_{A}^{-d},\left(a_{j} m_{j}^{\delta} e_{A}^{-d}\right)_{j \in \mathrm{C} J}\right)
$$

for all $\left(s_{1}, \ldots, s_{r}\right)$. Since $A$ is very ample and $\rho \geqslant k$, the power $A^{\rho}$ generates $k$-jets at every point $x \in Z$, and thus the morphisms

$$
H^{0}\left(Z, A^{\rho}\right) \rightarrow J^{k} \mathcal{O}_{Z, x}, \quad a \mapsto a m_{j}^{\delta} e_{A}^{-d} \quad \text { and } \quad H^{0}\left(Z, A^{k}\right) \rightarrow J^{k} \mathcal{O}_{Z, x}, \quad s \mapsto s \hat{\tau}_{j}^{d-k} e_{A}^{-d}
$$

are surjective. Lemma 12.25 applied with $r=k+c-N$ and $(p, m)$ replaced by $(k+1, N+1)$ implies that the codimension of families $a=\left(a_{0}, \ldots, a_{N}\right) \in S=H^{0}\left(Z, A^{\rho}\right)^{\oplus(N+1)}$ for which $\sigma_{k, s, \hat{\tau}, a, J}(\xi)=0$ for all choices of $s, \hat{\tau}$ and $J$ is at least $(c+1)(k+c-N+1)$, i.e. the dimension is at most $\operatorname{dim} S-(c+1)(k+c-N+1)$. When we let $\xi$ vary over $\widehat{Z}_{k}^{\prime}$ which has dimension $(n+1)+k n$ and take into account the fibration $(a, \xi) \mapsto \xi$, the dimension estimate of Proposition 12.26 follows. Under the assumption

$$
(c+1)(k+c-N+1)>n+1+k n
$$

we have $\operatorname{dim} \Gamma<\operatorname{dim} S$, and so the image of the projection $\Gamma \rightarrow S,(a, \xi) \mapsto a$, is a constructible algebraic subset distinct from $S$. This concludes the proof.

Our final goal is to completely eliminate the base locus. Proposition 12.26 indicates that we have to pay attention to the intersections $X_{a} \cap \tau_{I}^{-1}(0)$. For $x \in Z$, we let $\mathcal{G}$ be the family of hyperplane sections $\tau_{I}=0$ that contain $x$. We introduce the set $P=\{0,1, \ldots, N\} \backslash \bigcup_{I \in \mathcal{G}} I$ and the smooth intersection

$$
Z_{\mathcal{G}}=Z \cap \bigcap_{I \in \mathcal{G}} \tau_{I}^{-1}(0)
$$

so that $N^{\prime}+1:=\operatorname{card} P \geqslant N+1-c \operatorname{card} \mathcal{G}$ and $\operatorname{dim} Z_{\mathcal{G}}=n+1-\operatorname{card} \mathcal{G}$. If $a \in U$ is such that $x \in X_{a}$, we also look at the intersection

$$
X_{\mathcal{G}, a}=X_{a} \cap \bigcap_{I \in \mathcal{G}} \tau_{I}^{-1}(0),
$$

which is a smooth hypersurface of $Z_{\mathcal{G}}$. In that situation, we consider Wronskians $W_{k, s, \hat{\tau}, a, J}$ as defined above, but we now take $J \subset P$, card $J=c$, СJ $=P \backslash J, r^{\prime}=k+c-N^{\prime}$. 
12.29. Lemma. In the above setting, if we assume $\delta>k$, the restriction $W_{k, s, \hat{\tau}, a, J \mid X_{\mathcal{G}, a}}$ is still divisible by a monomial $\hat{\tau}^{\alpha_{J}} \tau^{\beta_{J}}$ such that

$$
\left|\alpha_{J}\right|+\left|\beta_{J}\right|=\left(k+c-N^{\prime}\right)(d-2 k)+\left(N^{\prime}+1-c\right)(\delta-k) b+(\delta-k) .
$$

Therefore, if

$$
p^{\prime}=\left|\alpha_{J}\right|+\left|\beta_{J}\right|-(k+1) d=(\delta-k)-\left(k+c-N^{\prime}\right) 2 k-\left(N^{\prime}+1+c\right)(k b+\rho)
$$

as in (12.17), we obtain again holomorphic sections

$$
\begin{aligned}
& \widetilde{W}_{k, s, \hat{\tau}, a, J\left\lceil X_{\mathcal{G}, a}\right.}:=\left(\hat{\tau}^{\alpha_{J}} \tau^{\beta_{J}}\right)^{-1} W_{k, s, \hat{\tau}, a, J\left\lceil X_{\mathcal{G}, a}\right.} \in H^{0}\left(X_{\mathcal{G}, a}, E_{k, k^{\prime}} T_{X}^{*} \otimes A^{-p^{\prime}}\right), \\
& \sigma_{k, s, \hat{\tau}, a, J \mid \pi_{k, 0}^{-1}\left(X_{\mathcal{G}, a}\right)} \in H^{0}\left(\pi_{k, 0}^{-1}\left(X_{\mathcal{G}, a}\right), \mu_{a, k}^{*}\left(\mathcal{O}_{X_{a, k}}\left(k^{\prime}\right) \otimes \pi_{k, 0}^{*} A^{-p^{\prime}}\right) \otimes \mathcal{O}_{\widehat{X}_{a, k}}\left(-F_{a, k}\right)\right) .
\end{aligned}
$$

Proof. The arguments are similar to those employed in the proof of Lemma 12.15. Let $f_{[k]} \in X_{a, k}$ be a $k$-jet such that $f(0) \in X_{\mathcal{G}, a}$ (the $k$-jet need not be entirely contained in $X_{\mathcal{G}, a}$ ). Putting $j_{0}=\min (\complement J)$, we observe that we have on $X_{\mathcal{G}, a}$ an identity

$$
a_{j_{0}} m_{j_{0}}^{\delta}=-\sum_{i \in P \backslash\left\{j_{0}\right\}} a_{i} m_{i}^{\delta}=-\sum_{i \in J} a_{i} m_{i}^{\delta}-\sum_{P \backslash\left(J \cup\left\{j_{0}\right\}\right)} a_{i} m_{i}^{\delta},
$$

because $m_{i}=\prod_{I \ni i} \tau_{I}=0$ on $X_{\mathcal{G}, a}$ for $i \in \mathcal{C} P=\bigcup_{I \in \mathcal{G}} I$ (one of the factors $\tau_{I}$ is such that $I \in \mathcal{G}$, so $\tau_{I}=0$ ). If we compose with a germ $t \mapsto f(t)$ such that $f(0) \in X_{\mathcal{G}, a}$ (even though $f$ does not necessarily lie entirely in $\left.X_{\mathcal{G}, a}\right)$, we get

$$
a_{j_{0}} m_{j_{0}}^{\delta}(f(t))=-\sum_{i \in J} a_{i} m_{i}^{\delta}(f(t))-\sum_{P \backslash\left(J \cup\left\{j_{0}\right\}\right)} a_{i} m_{i}^{\delta}(f(t))+O\left(t^{k+1}\right)
$$

as soon as $\delta>k$. Hence we have an equality for all derivatives $D^{\ell}(\bullet), \ell \leqslant k$ at $t=0$, and

$$
W_{k, s, \hat{\tau}, a, J\left\lceil X_{\mathcal{G}, a}\right.}\left(f_{[k]}\right)=-\sum_{i \in J} W_{k}\left(s_{1} \hat{\tau}_{1}^{d-k}, \ldots, s_{r^{\prime}} \hat{\tau}_{r^{\prime}}^{d-k}, a_{i} m_{i}^{\delta},\left(a_{j} m_{j}^{\delta}\right)_{j \in P \backslash\left(J \cup\left\{j_{0}\right\}\right)}\right)_{\left\lceil X_{\mathcal{G}, a}\right.}\left(f_{[k]}\right) .
$$

Then, again, $\tau_{J}^{\delta-k}$ is a new additional common factor of all terms in the sum, and we conclude as in Lemma 12.15 and Corollary 12.16.

Now, we analyze the base locus of these new sections on

$$
\bigcup_{a \in U} \mu_{a, k}^{-1} \pi_{k, 0}^{-1}\left(X_{\mathcal{G}, a}\right) \subset \mu_{k}^{-1} \pi_{k, 0}^{-1}\left(Z_{\mathcal{G}}\right) \subset \widehat{Z}_{k} .
$$

As $x$ runs in $Z_{\mathcal{G}}$ and $N^{\prime}<N$, Lemma 12.25 shows that (12.28) can be replaced by the less demanding condition

$$
(c+1)\left(k+c-N^{\prime}+1\right)>n+1-\operatorname{card} \mathcal{G}+k n=\operatorname{dim} \mu_{k}^{-1} \pi_{k, 0}^{-1}\left(Z_{\mathcal{G}}\right) .
$$

A proof entirely similar to that of Proposition 12.26 shows that for a generic choice of $a \in U$, the base locus of these sections on $\widehat{X}_{\mathcal{G}, a, k}$ projects onto $\bigcup_{I \in \complement \mathcal{G}} X_{\mathcal{G}, a} \cap \tau_{I}^{-1}(0)$. Arguing inductively on card $\mathcal{G}$, the base locus can be shrinked step by step down to empty set (but it is in fact sufficient to stop when $X_{\mathcal{G}, a} \cap \tau_{I}^{-1}(0)$ reaches dimension 0).

\section{F. Nefness and ampleness of appropriate taUtological line Bundles}

At this point, we have produced a smooth family $\mathcal{X}_{S} \rightarrow U \subset S$ of particular hypersurfaces in $Z$, namely $X_{a}=\left\{\sigma_{a}(z)=0\right\}, a \in U$, for which a certain "tautological" line bundle has an empty base locus for sufficiently general coefficients:

12.30. Corollary. Under condition (12.28) and the hypothesis $p>0$ in (12.17), the following properties hold.

(a) The line bundle

$$
\mathcal{L}_{a}:=\mu_{a, k}^{*}\left(\mathcal{O}_{X_{a, k}}\left(k^{\prime}\right) \otimes \pi_{k, 0}^{*} A^{-1}\right) \otimes \mathcal{O}_{\widehat{X}_{a, k}}\left(-F_{a, k}\right)
$$


is nef on $\widehat{X}_{a, k}$ for general $a \in U$, i.e. for $a \in U^{\prime} \subset U$, where $U^{\prime}$ is a dense Zariski open subset. (b) Let $\Delta_{a}=\sum_{2 \leqslant \ell \leqslant k} \lambda_{\ell} D_{a, \ell}$ be a positive rational combination of vertical divisors of the Semple tower and $q \in \mathbb{N}, q \gg 1$, an integer such that

$$
\mathcal{L}_{a}^{\prime}:=\mathcal{O}_{X_{a, k}}(1) \otimes \mathcal{O}_{a, k}\left(-\Delta_{a}\right) \otimes \pi_{k, 0}^{*} A^{q}
$$

is ample on $X_{a, k}$. Then the $\mathbb{Q}$-line bundle

$$
\mathcal{L}_{a, \varepsilon, \eta}^{\prime}:=\mu_{a, k}^{*}\left(\mathcal{O}_{X_{a, k}}\left(k^{\prime}\right) \otimes \mathcal{O}_{X_{a, k}}\left(-\varepsilon \Delta_{a}\right) \otimes \pi_{k, 0}^{*} A^{-1+q \varepsilon}\right) \otimes \mathcal{O}_{\widehat{X}_{a, k}}\left(-(1+\varepsilon \eta) F_{a, k}\right)
$$

is ample on $\widehat{X}_{a, k}$ for $a \in U^{\prime}$, for some $q \in \mathbb{N}$ and $\varepsilon, \eta \in \mathbb{Q}_{>0}$ arbitrarily small.

Proof. (a) This would be obvious if we had global sections generating $\mathcal{L}_{a}$ on the whole of $\widehat{X}_{a, k}$, but our sections are only defined on a stratification of $\widehat{X}_{a, k}$. In any case, if $C \subset \widehat{X}_{a, k}$ is an irreducible curve, we take a maximal family $\mathcal{G}$ such that $C \subset X_{\mathcal{G}, a, k}$. Then, by what we have seen, for $a \in U$ general enough, we can find global sections of $\mathcal{L}_{a}$ on $\widehat{X}_{\mathcal{G}, a, k}$ such that $C$ is not contained in their base locus. Hence $\mathcal{L}_{a} \cdot C \geqslant 0$ and $\mathcal{L}_{a}$ is nef for $a$ in a dense Zariski open set $U^{\prime} \subset U$.

(b) The existence of $\Delta_{a}$ and $q$ follows from Proposition 7.19 and Corollary 7.21, which even provide universal values for $\lambda_{\ell}$ and $q$. After taking the blow up $\mu_{a, k}: \widehat{X}_{a, k} \rightarrow X_{a, k}$ (cf. (12.7)), we infer that

$$
\mathcal{L}_{a, \eta}^{\prime}:=\mu_{a, k}^{*} \mathcal{L}^{\prime} \otimes \mathcal{O}_{\widehat{X}_{a, k}}\left(-\eta F_{a, k}\right)=\mu_{a, k}^{*}\left(\mathcal{O}_{X_{a, k}}(1) \otimes \mathcal{O}_{X_{a, k}}\left(-\Delta_{a}\right) \otimes \pi_{k, 0}^{*} A^{q}\right) \otimes \mathcal{O}_{\widehat{X}_{a, k}}\left(-\eta F_{a, k}\right)
$$

is ample for $\eta>0$ small. The result now follows by taking a combination

$$
\mathcal{L}_{a, \varepsilon, \eta}=\mathcal{L}_{a}^{1-\varepsilon / k^{\prime}} \otimes\left(\mathcal{L}_{a, \eta}^{\prime}\right)^{\varepsilon} .
$$

12.31. Corollary. Let $\mathcal{X} \rightarrow \Omega$ be the universal family of hypersurfaces $X_{\sigma}=\{\sigma(z)=0\}, \sigma \in \Omega$, where $\Omega \subset P\left(H^{0}\left(Z, A^{d}\right)\right)$ is the dense Zariski open set over which the family is smooth. On the "Wronskian blow-up" $\widehat{X}_{\sigma, k}$ of $X_{\sigma, k}$, let us consider the line bundle

$$
\mathcal{L}_{\sigma, \varepsilon, \eta}:=\mu_{\sigma, k}^{*}\left(\mathcal{O}_{X_{\sigma, k}}\left(k^{\prime}\right) \otimes \mathcal{O}_{X_{\sigma, k}}\left(-\varepsilon \Delta_{\sigma}\right) \otimes \pi_{k, 0}^{*} A^{-1+q \varepsilon}\right) \otimes \mathcal{O}_{\widehat{X}_{\sigma, k}}\left(-(1+\varepsilon \eta) F_{\sigma, k}\right)
$$

associated with the same choice of constants as in Cor. 12.30. Then $\mathcal{L}_{\sigma, \varepsilon, \eta}^{\prime}$ is ample on $\widehat{X}_{\sigma, k}$ for $\sigma$ in a dense Zariski open set $\Omega^{\prime} \subset \Omega$.

Proof. By Cor. 12.30 (b), we can find $\sigma_{0} \in H^{0}\left(Z, A^{d}\right)$ such that $X_{\sigma_{0}}=\sigma_{0}^{-1}(0)$ is smooth and $\mathcal{L}_{\sigma_{0}, \varepsilon, \eta}^{m}$ is an ample line bundle on $\widehat{X}_{\sigma_{0}, k}\left(m \in \mathbb{N}^{*}\right)$. As ampleness is a Zariski open condition, we conclude that $\mathcal{L}_{\sigma, \varepsilon, \eta}^{m}$ remains ample for a general section $\sigma \in H^{0}\left(Z, A^{d}\right)$, i.e. for $[\sigma]$ in some Zariski open set $\Omega^{\prime} \subset \Omega$. Since $\mu_{\sigma, k}\left(F_{\sigma, k}\right)$ is contained in the vertical divisor of $X_{\sigma, k}$, we conclude by Theorem 8.8 that $X_{\sigma}$ is Kobayashi hyperbolic for $[\sigma] \in \Omega$.

\section{G. FinAl CONCLUSion AND COMPUtATion OF DEGREe BOUNDS}

At this point, we fix our integer parameters to meet all conditions that have been found. We must have $N \geqslant c(n+1)$ by Lemma 12.12, and for such a large value of $N$, condition (12.28) can hold only when $c \geqslant n$, so we take $c=n$ and $N=n(n+1)$. Inequality (12.28) then requires $k$ large enough, $k=n^{3}+n^{2}+1$ being the smallest possible value. We find

$$
b=\left(\begin{array}{c}
N \\
c-1
\end{array}\right)=\left(\begin{array}{c}
n^{2}+n \\
n-1
\end{array}\right)=n \frac{\left(n^{2}+n\right) \ldots\left(n^{2}+2\right)}{n !} .
$$

We have $n^{2}+k=n^{2}\left(1+k / n^{2}\right)<n^{2} \exp \left(k / n^{2}\right)$ and by Stirling's formula, $n !>\sqrt{2 \pi n}(n / e)^{n}$. Therefore

$$
b<\frac{n^{2 n-1} \exp \left((2+\cdots+n) / n^{2}\right)}{\sqrt{2 \pi n}(n / e)^{n}}<\frac{e^{n+\frac{1}{2}+\frac{1}{2 n}}}{\sqrt{2 \pi}} n^{n-\frac{3}{2}} .
$$


Finally, we divide $d-k$ by $b$, get in this way $d-k=b \delta+\lambda, 0 \leqslant \lambda<b$, and put $\rho=\lambda+k \geqslant k$. Then $\delta+1 \geqslant(d-k+1) / b$ and formula (12.17) yields

$$
\begin{aligned}
p & =(\delta-k)-\left(n^{3}+1\right) 2 k-\left(n^{2}+2 n+1\right)(k b+\rho) \\
& \geqslant(d-k+1) / b-1-\left(2 n^{3}+3\right) k-\left(n^{2}+2 n+1\right)(k b+k+b-1) .
\end{aligned}
$$

Therefore $p>0$ is achieved as soon as

$$
d \geqslant d_{n}=k+b\left(1+\left(2 n^{3}+3\right) k+\left(n^{2}+2 n+1\right)(k b+k+b-1)\right),
$$

where

$$
k=n^{3}+n^{2}+1, \quad b=\left(\begin{array}{c}
n^{2}+n \\
n-1
\end{array}\right) .
$$

The dominant term in $d_{n}$ is $k\left(n^{2}+2 n+1\right) b^{2} \sim e^{2 n+1} n^{2 n+2} / 2 \pi$. By more precise numerical calculations and Stirling's asymptotic expansion, one can show in fact that $d_{n} \leqslant\left\lfloor(n+4)(e n)^{2 n+1} / 2 \pi\right\rfloor$ for $n \geqslant 4$ (which is also an equivalent and a close approximation as $n \rightarrow+\infty$ ), while $d_{1}=61$, $d_{2}=6685, d_{3}=2825761$. We can now state the main result of this section.

12.32. Theorem. Let $Z$ be a projective $(n+1)$-dimensional manifold and $A$ a very ample line bundle on $Z$. Then, for a general section $\sigma \in H^{0}\left(Z, A^{d}\right)$ and $d \geqslant d_{n}$, the hypersurface $X_{\sigma}=\sigma^{-1}(0)$ is Kobayashi hyperbolic, and in fact, algebraically jet hyperbolic in the sense of Definition 11.11. The bound $d_{n}$ for the degree can be taken to be

$$
d_{n}=\left\lfloor(n+4)(e n)^{2 n+1} / 2 \pi\right\rfloor \text { for } n \geqslant 4,
$$

and for $n \leqslant 3$, one can take $d_{1}=4, d_{2}=6685, d_{3}=2825761$.

A simpler (and less refined) choice is $\tilde{d}_{n}=\left\lfloor\frac{1}{3}(e n)^{2 n+2}\right\rfloor$, which is valid for all $n$. These bounds are only slightly weaker than the ones found by Ya Deng in [Deng16, Deng17], namely $\tilde{d}_{n}=O\left(n^{2 n+6}\right)$.

Proof. The bound $d_{1}=4$ (instead the insane value $d_{1}=61$ ) can be obtained in an elementary way by adjunction: sections of $A$ can be used to embed any polarized surface $(Z, A)$ in $\mathbb{P}^{N}$ (one can always take $N=5$ ), and we have $K_{X_{\sigma}}=K_{Z \backslash X_{\sigma}} \otimes A^{d}$, along with a surjective morphism $\Omega_{\mathbb{P}^{N}}^{2} \rightarrow K_{Z}$. As $\Omega_{\mathbb{P}^{N}}^{2} \otimes \mathcal{O}(3)=\Lambda^{N-2}\left(T_{\mathbb{P}^{N}} \otimes \mathcal{O}(-1)\right)$ is generated by sections, this implies that $K_{Z} \otimes A^{3}$ is also generated by sections, and hence $K_{X_{\sigma}}$ is ample for $d \geqslant 4$.

\section{H. FURTHER COMMENTS}

12.33. Our bound $d_{n}$ is rather large, but just as in Ya Deng's effective approach of Brotbek's theorem [Deng17], the bound holds for a property that looks substantially stronger than hyperbolicity, namely the ampleness of the pull-back of some (twisted) jet bundle $\mu_{k}^{*} \mathcal{O}_{\widehat{X}_{k}}\left(a_{\bullet}\right) \otimes \mathcal{O}_{\widehat{X}_{k}}\left(-F_{k}^{\prime}\right)$. Section 11.B provides much weaker conditions for hyperbolicity, but checking them is probably more involved.

12.34. After these notes were written, Riedl and Yang [RiYa18] proved the important and somewhat surprising result that the lower bound estimates $d_{\mathrm{GG}}(n)$ and $d_{\mathrm{Kob}}(n)$, respectively for the Green-Griffiths-Lang and Kobayashi conjectures for general hypersurfaces in $\mathbb{P}^{n+1}$, can be related by $d_{\mathrm{Kob}}(n):=d_{\mathrm{GG}}(2 n-2)$. This should be understood in the sense that a solution of the generic $(2 n-2)$-dimensional Green-Griffiths conjecture for $d \geqslant d_{\mathrm{GG}}(2 n-2)$ implies a solution of the $n$-dimensional Kobayashi conjecture for the same lower bound. We refer to [RiYa18] for the precise statement, which requires an ad hoc assumption on the algebraic dependence of the Green-Griffiths locus with respect to a variation of coefficients in the defining polynomials. In combination with [DMR10], this gives a completely new proof of the Kobayashi conjecture, and the order 1 bound $d_{\mathrm{GG}}(n)=O\left(\exp \left(n^{1+\varepsilon}\right)\right)$ of [Dem12] implies a similar bound $d_{\mathrm{Kob}}(n)=O\left(\exp \left(n^{1+\varepsilon}\right)\right)$ for the Kobayashi conjecture - just a little bit weaker than what our direct proof gave (Theorem 12.32). In [MeTa19], Merker and Ta were able to improve the Green-Griffiths bound to $d_{\mathrm{GG}}(n)=o\left((\sqrt{n} \log n)^{n}\right)$, using a strengthening of Darondeau's estimates [Dar16a, Dar16b], along 
with very delicate calculations. The Riedl-Yang result then implies $d_{\text {Kob }}(n)=O\left((n \log n)^{n+1}\right)$, which is the best bound known at this time.

12.35. In [Ber18], G. Bérczi stated a positivity conjecture for Thom polynomials of Morin singularities (see also [BeSz12]), and showed that it would imply a polynomial bound $d_{n}=2 n^{9}+1$ for the generic hyperbolicity of hypersurfaces.

12.36. In the unpublished preprint [Dem15], we introduced an alternative strategy for the proof of the Kobayashi conjecture which appears to be still incomplete at this point. We nevertheless hope that a refined version could one day lead to linear bounds such as $d_{\mathrm{Kob}}(n)=2 n+1$. The rough idea was to establish a $k$-jet analogue of Claire Voisin's proof [Voi96] of the Clemens conjecture. Unfortunately, Lemma 5.1.18 as stated in [Dem15] is incorrect - the assertion concerning the $\Delta$ divisor introduced there simply does not hold. It is however conceivable that a weaker statement holds, in the form of a control of the degree of the divisor $\Delta$, and in a way that would still be sufficient to imply similar consequences for the generic positivity of tautological jet bundles, as demanded e.g. in section 11.B.

\section{REFERENCES}

[Ahl41] Ahlfors, L.V.: The theory of meromorphic curves. Acta Soc. Sci. Finn. Nova Ser. A., 3 (1941), $1-31$.

[ASS97] Arrondo, E., Sols, I., Speiser, R.: Global moduli for contacts. Ark. Mat. 35 (1997), 1-57.

[AzSu80] Azukawa, K. and Suzuki, M. Some examples of algebraic degeneracy and hyperbolic manifolds. Rocky Mountain J. Math. 10 (1980), 655-659.

[Ber15] Bérczi, G.: Towards the Green-Griffiths-Lang conjecture via equivariant localisation. arXiv: math.AG/1509.03406.

[Ber18] Bérczi, G.: Thom polynomials and the Green-Griffiths-Lang conjecture for hypersurfaces with polynomial degree. Intern. Math. Res. Not. https://doi.org/10.1093/imrn/rnx332 (2018), 1-56.

[BeKi12] Bérczi, G., Kirwan, F.: A geometric construction for invariant jet differentials. Surveys in Diff. Geom., Vol XVII (2012), 79-125.

[BeSz12] Bérczi, G., Szenes, A.: Thom polynomials of Morin singularities. Annals of Math. 175 (2012), 567-629.

[Blo26a] Bloch, A.: Sur les systèmes de fonctions uniformes satisfaisant à l'équation d'une variété algébrique dont l'irrégularité dépasse la dimension. J. Math. ¿Pures Appl., 5 (1926), 19-66.

[Blo26b] Bloch, A.: Sur les systèmes de fonctions holomorphes à variétés linéaires lacunaires. Ann. Ecole Normale, 43 (1926), 309-362.

[Bog77] Bogomolov, F.A.: Families of curves on a surface of general type. Soviet Math. Dokl. 236 (1977), 1294-1297.

[Bog79] Bogomolov, F.A.: Holomorphic tensors and vector bundles on projective manifolds. Math. USSR Izvestija 13/3 (1979), 499-555.

[Bon93] Bonavero, L.: Inégalités de Morse holomorphes singulières. C. R. Acad. Sci. Paris Sér. I Math. 317 (1993) 1163-1166.

[Bro78] Brody, R.: Compact manifolds and hyperbolicity. Trans. Amer. Math. Soc. 235 (1978), 213-219.

[BrGr77] Brody, R., Green, M.: A family of smooth hyperbolic hypersurfaces in $\mathbb{P}^{3}$. Duke Math. J. 44 (1977), 873-874.

[Brot17] Brotbek, D.: On the hyperbolicity of general hypersurfaces. Publications mathématiques de l'IHÉS, 126 (2017), 1-34.

[BrDa17] Brotbek, D., Darondeau, L. Complete intersection varieties with ample cotangent bundles. Invent. Math. 212 (2018), 913-940.

[BR90] Brückmann P., Rackwitz, H.-G.: T-Symmetrical Tensor Forms on Complete Intersections. Math. Ann. 288 (1990), 627-635.

[Bru02] Brunella, M.: Courbes entières dans les surfaces algébriques complexes (d'après McQuillan, Demailly-El Goul, ...). Séminaire Bourbaki, Vol. 2000/2001. Astérisque 282 (2002), Exp. No. 881, 39-61. 
[Bru03] Brunella, M.: Plurisubharmonic variation of the leafwise Poincaré metric. Int. J. Math. 14 (2003) 139-151.

[Bru05] Brunella, M.: On the plurisubharmonicity of the leafwise Poincaré metric on projective manifolds. J. Math. Kyoto Univ. 45 (2005) 381-390.

[Bru06] Brunella, M.: A positivity property for foliations on compact Kähler manifolds. Internat. J. Math. 17 (2006) 35-43.

[Can00] Cantat, S.: Deux exemples concernant une conjecture de Serge Lang. C. R. Acad. Sci. Paris Sér. I Math. 330 (2000), 581-586.

[CaGr72] Carlson, J.A., Griffiths, P.A.: A defect relation for equidimensional holomorphic mappings between algebraic varieties. Ann. Math. 95 (1972), 557-584.

[Cart28] Cartan, H.: Sur les systèmes de fonctions holomorphes à variétés linéaires lacunaires et leurs applications, Thèse, Paris. Ann. Ecole Normale, 45 (1928), 255-346.

[Carl72] Carlson, J.A.: Some degeneracy theorems for entire functions with values in an algebraic variety. Trans. Amer. Math. Soc. 168 (1972), 273-301.

[CFZ17] Ciliberto, C., Flamini, F., Zaidenberg, M.: A remark on the intersection of plane curves. math.AG, arXiv:1704.00320, 16p.

[CKM88] Clemens, H., Kollár, J., Mori, S.: Higher dimensional complex geometry. Astérisque $166,1988$.

[Cle86] Clemens, H.: Curves on generic hypersurfaces. Ann. Sci. Ec. Norm. Sup. 19 (1986), 629-636.

[ClR04] Clemens, H., Ran, Z.: Twisted genus bounds for subvarieties of generic hypersurfaces. Amer. J. Math. 126 (2004), 89-120.

[CoKe94] Colley, S.J., Kennedy, G.: The enumeration of simultaneous higher order contacts between plane curves. Compositio Math. 93 (1994), 171-209.

[Coll88] Collino, A.: Evidence for a conjecture of Ellingsrud and Strømme on the Chow ring of $\mathbf{H i l b}_{d}\left(\mathbb{P}^{2}\right)$. Illinois J. Math. 32 (1988), 171-210.

[CoGr76] Cowen, M., Griffiths, P.A.: Holomorphic curves and metrics of negative curvature. J. Analyse Math. 29 (1976), 93-153.

[Dar14] Darondeau, L.: Effective algebraic degeneracy of entire curves in complements of smooth projective hypersurfaces. preprint Univ. Paris Sud Orday, arXiv:1402.1396.

[Dar16a] Darondeau, L.: Fiber integration on the Demailly tower. Ann. Inst. Fourier 66 (2016), $29-54$.

[Dar16b] Darondeau, L.: On the logarithmic Green-Griffiths conjecture. Int. Math. Res. Not. (2016), no. 6, 1871-1923.

[DPP06] Debarre, O., Pacienza, G., Păun, M.: Non-deformability of entire curves in projective hypersurfaces of high degree. Ann. Inst. Fourier (Grenoble) 56 (2006) 247-253.

[Dem85] Demailly, J.-P.: Champs magnétiques et inégalités de Morse pour la d" -cohomologie. Ann. Inst. Fourier (Grenoble) 35 (1985) 189-229.

[Dem90a] Demailly, J.-P.: Cohomology of q-convex spaces in top degrees. Math. Zeitschrift 203 (1990) 283-295.

[Dem90b] Demailly, J.-P.: Singular hermitian metrics on positive line bundles. Proceedings of the Bayreuth conference "Complex algebraic varieties", April 2-6, 1990, edited by K. Hulek, T. Peternell, M. Schneider, F. Schreyer, Lecture Notes in Math. n 1507, Springer-Verlag (1992), 87-104.

[Dem92] Demailly, J.-P.: Regularization of closed positive currents and Intersection Theory. J. Alg. Geom. 1 (1992) 361-409.

[Dem94] Demailly, J.-P.: $L^{2}$ vanishing theorems for positive line bundles and adjunction theory. alggeom/9410022; Lecture Notes of the CIME Session "Transcendental methods in Algebraic Geometry", Cetraro, Italy, July 1994, Ed. F. Catanese, C. Ciliberto, Lecture Notes in Math., Vol. 1646, CIME Found. Subser., Springer-Verlag, 1-97.

[Dem95] Demailly, J.-P.: Algebraic criteria for Kobayashi hyperbolic projective varieties and jet differentials. AMS Summer School on Algebraic Geometry, Santa Cruz 1995, Proc. Symposia in Pure Math., vol. 062.2, ed. by J. Kollár and R. Lazarsfeld, Amer. Math. Soc., Providence, RI (1997), 285-360.

[Dem97] Demailly, J.-P.: Variétés hyperboliques et équations différentielles algébriques. Gaz. Math. 73 (juillet 1997) 3-23.

[Dem07a] Demailly, J.-P.: Structure of jet differential rings and holomorphic Morse inequalities. Talk at the CRM Workshop "The geometry of holomorphic and algebraic curves in complex algebraic varieties", Montréal, May 2007. 
[Dem07b] Demailly, J.-P.: On the algebraic structure of the ring of jet differential operators. Talk at the conference "Effective aspects of complex hyperbolic varieties", Aber Wrac'h, France, September 10-14, 2007.

[Dem11] Demailly, J.-P.: Holomorphic Morse Inequalities and the Green-Griffiths-Lang Conjecture. Pure and Applied Math. Quarterly 7 (2011), 1165-1208.

[Dem12] Demailly, J.-P.: Hyperbolic algebraic varieties and holomorphic differential equations. expanded version of the lectures given at the annual meeting of VIASM, Acta Math. Vietnam. 37 (2012), 441-512.

[Dem14] Demailly, J.-P.: Towards the Green-Griffiths-Lang conjecture. Conference "Analysis and Geometry", Tunis, March 2014, in honor of Mohammed Salah Baouendi, ed. by A. Baklouti, A. El Kacimi, S. Kallel, N. Mir, Springer Proc. Math. Stat., 127, Springer-Verlag, 2015, 141-159.

[Dem15] Demailly, J.-P.: Proof of the Kobayashi conjecture on the hyperbolicity of very general hypersurfaces. arXiv:1501.07625, math.CV, unpublished.

[DeEG97] Demailly, J.-P., El Goul, J.: Connexions méromorphes projectives et variétés algébriques hyperboliques. C. R. Acad. Sci. Paris, Série I, 324 (1997), 1385-1390.

DeEG00 Demailly, J.-P., El Goul, J.: Hyperbolicity of generic surfaces of high degree in projective 3-space. Amer. J. Math. 122 (2000), 515-546.

[DeLS94] Demailly, J.-P., Lempert L., Shiffman, B.: Algebraic approximation of holomorphic maps from Stein domains to projective manifolds. Duke Math. J. 76 (1994) 333-363.

DePS94 Demailly, J.-P., Peternell, Th., Schneider, M.: Compact complex manifolds with numerically effective tangent bundles. Algebraic Geometry 3 (1994), 295-345.

[Deng16] Deng, Ya.: Effectivity in the hyperbolicity related problems. Chap. 4 of the PhD memoir "Generalized Okounkov Bodies, Hyperbolicity-Related and Direct Image Problems" defended on June 26, 2017 at Université Grenoble Alpes, Institut Fourier, arXiv:1606.03831, math.CV.

[Deng17] Deng, Ya.: On the Diverio-Trapani Conjecture arXiv:1703.07560, math.CV.

[DGr91] Dethloff, G., Grauert, H.: On the infinitesimal deformation of simply connected domains in one complex variable. International Symposium in Memory of Hua Loo Keng, Vol. II (Beijing, 1988), Springer, Berlin, (1991), 57-88.

[DLu01] Dethloff, G., Lu, S.: Logarithmic jet bundles and applications. Osaka J. Math. 38 (2001), $185-237$.

[DTH16a] Dinh, Tuan Huynh: Examples of hyperbolic hypersurfaces of low degree in projective spaces. Int. Math. Res. Not. 18 (2016) 5518-5558.

[DTH16b] Dinh, Tuan Huynh: Construction of hyperbolic hypersurfaces of low degree in $\mathbb{P}^{n}(\mathbb{C})$. Int. J. Math. 27 (2016) 1650059 (9 pages).

[Div08] Diverio, S.: Differential equations on complex projective hypersurfaces of low dimension. Compos. Math. 144 (2008) 920-932.

[Div09] Diverio, S.: Existence of global invariant jet differentials on projective hypersurfaces of high degree. Math. Ann. 344 (2009) 293-315.

[DMR10] Diverio, S., Merker, J., Rousseau, E.: Effective algebraic degeneracy. Invent. Math. 180 (2010) $161-223$.

[DR11] Diverio, S., Rousseau, E.: A survey on hyperbolicity of projective hypersurfaces. Publ. Mat. IMPA, Inst. Nac. Mat. Pura. Apl., Rio de Janeiro, 2011.

[DR15] Diverio, S., Rousseau, E.: The exceptional set and the GreenGriffiths locus do not always coincide. Enseign. Math. 61 (2015) 417-452.

[DT10] Diverio, S., Trapani, S.: A remark on the codimension of the Green-Griffiths locus of generic projective hypersurfaces of high degree. J. Reine Angew. Math. 649 (2010) 55-61.

[Dol81] Dolgachev, I.: Weighted projective varieties. Proceedings Polish-North Amer. Sem. on Group Actions and Vector Fields, Vancouver, 1981, J.B. Carrels editor, Lecture Notes in Math. 956, Springer-Verlag (1982), 34-71.

[Duv04] Duval J.: Une sextique hyperbolique dans $\mathbb{P}^{3}(\mathbb{C})$. Math. Ann. 330 (2004) 473-476.

[Duv08] Duval J.: Sur le lemme de Brody. Invent. Math. 173 (2008), 305-314.

[Ein88] Ein L.: Subvarieties of generic complete intersections. Invent. Math. 94 (1988), $163-169$.

[Ein91] Ein L.: Subvarieties of generic complete intersections, II. Math. Ann. 289 (1991), 465-471.

[EG96] El Goul, J.: Algebraic families of smooth hyperbolic surfaces of low degree in $\mathbb{P}_{\mathbb{C}}^{3}$. Manuscripta Math. 90 (1996), 521-532. 
[EG97] El Goul, J.: Propriétés de négativité de courbure des variétés algébriques hyperboliques. Thèse de Doctorat, Univ. de Grenoble I (1997).

[Fuj72] Fujimoto H.: On holomorphic maps into a taut complex space Nagoya Math. J. 46 (1972), 49-61.

[Fuj01] Fujimoto, H.: A family of hyperbolic hypersurfaces in the complex projective space. Complex Variables, Theory Appl. 43, no. 3-4, (2001) 273-283.

[Fuji94] Fujita, T.: Approximating Zariski decomposition of big line bundles. Kodai Math. J. 17 (1994) $1-3$.

[Ghe41] Gherardelli, G.: Sul modello minimo della varieta degli elementi differenziali del $2^{\circ}$ ordine del piano projettivo. Atti Accad. Italia. Rend., Cl. Sci. Fis. Mat. Nat. (7) 2 (1941), 821-828.

[Gra89] Grauert, H.: Jetmetriken und hyperbolische Geometrie. Math. Zeitschrift 200 (1989), 149-168.

[GRe65] Grauert, H., Reckziegel, H.: Hermitesche Metriken und normale Familien holomorpher Abbildungen. Math. Zeitschrift 89 (1965), 108-125.

[GrGr80] Green, M., Griffiths, P.A.: Two applications of algebraic geometry to entire holomorphic mappings. The Chern Symposium 1979, Proc. Internal. Sympos. Berkeley, CA, 1979, SpringerVerlag, New York (1980), 41-74.

[Gri71] Griffiths, P.: Holomorphic mappings into canonical algebraic varieties. Ann. of Math. 98 (1971), $439-458$.

[Har77] Hartshorne, R.: Algebraic geometry. Gard. Texts in Math., 52, Springer-Verlag, 1977.

[Hir64] Hironaka, H.: Resolution of singularities of an algebraic variety over a field of characteristic zero. Ann. of Math. 79 (1964) 109-326.

[HVX17] Huynh, D.T., Vu, D.V., Xie, S.Y.: Entire holomorphic curves into projective plane intersecting few generic algebraic curves. math.AG, arXiv:1704.03358, 9p.

[Kaw80] Kawamata, Y.: On Bloch's conjecture. Invent. Math. 57 (1980), 97-100.

[Kob70] Kobayashi, S.: Hyperbolic manifolds and holomorphic mappings. Pure Appl. Math., 2, Marcel Dekker, New York, NY, 1970.

[Kob75] Kobayashi, S.: Negative vector bundles and complex Finsler structures. Nagoya Math. J. 57 (1975), 153-166.

[Kob76] Kobayashi, S.: Intrinsic distances, measures and geometric function theory. Bull. Amer. Math. Soc. 82 (1976), 357-416.

[Kob80] Kobayashi, S.: The first Chern class and holomorphic tensor fields. J. Math. Soc. Japan 32 (1980), 325-329.

[Kob81] Kobayashi, S.: Recent results in complex differential geometry. Jber. dt. Math.-Verein. 83 (1981), 147-158.

[Kob98] Kobayashi, S.: Hyperbolic complex spaces. Grundlehren der Mathematischen Wissenschaften, volume 318, Springer-Verlag, Berlin, 1998.

[KobO71] Kobayashi, S., Ochiai, T.: Mappings into compact complex manifolds with negative first Chern class. J. Math. Soc. Japan 23 (1971), 137-148.

[KobO75] Kobayashi, S., Ochiai, T.: Meromorphic mappings onto compact complex spaces of general type. Invent. Math. 31 (1975), 7-16.

[KobR91] Kobayashi, R.: Holomorphic curves into algebraic subvarieties of an abelian variety. Internat. J. Math. 2 (1991), 711-724.

[LaTh96] Laksov, D., Thorup, A.: These are the differentials of order $n$. Trans. Amer. Math. Soc. 351 (1999), 1293-1353.

[Lang86] Lang, S.: Hyperbolic and Diophantine analysis. Bull. Amer. Math. Soc. 14 (1986), 159-205.

[Lang87] Lang, S.: Introduction to complex hyperbolic spaces. Springer-Verlag, New York (1987).

[Lu96]

Lu, S.S.Y.: On hyperbolicity and the Green-Griffiths conjecture for surfaces. Geometric Complex Analysis, ed. by J. Noguchi et al., World Scientific Publishing Co. (1996), 401-408.

[LuMi95] Lu, S.S.Y., Miyaoka, Y.: Bounding curves in algebraic surfaces by genus and Chern numbers. Math. Research Letters 2 (1995), 663-676.

[LuMi96] Lu, S.S.Y., Miyaoka, Y.: Bounding codimension one subvarieties and a general inequality between Chern numbers. Amer. J. of Math. 119 (1997), 487-502.

[LuWi12] Lu, S.S.Y., Winkelmann, J.: Quasiprojective varieties admitting Zariski dense entire holomorphic curves. Forum Math. 24 (2012), 399-418. 
[LuYa90] Lu, S.S.Y., Yau, S.T.: Holomorphic curves in surfaces of general type. Proc. Nat. Acad. Sci. USA, 87 (January 1990), 80-82.

[MaNo96] Masuda, K., Noguchi, J.: A construction of hyperbolic hypersurface of $\mathbb{P}^{n}(\mathbb{C})$. Math. Ann. 304 (1996), 339-362.

[McQ96] McQuillan, M.: A new proof of the Bloch conjecture. J. Alg. Geom. 5 (1996), 107-117.

[McQ98] McQuillan, M.: Diophantine approximation and foliations. Inst. Hautes Études Sci. Publ. Math. 87 (1998), 121-174.

[McQ99] McQuillan, M.: Holomorphic curves on hyperplane sections of 3-folds. Geom. Funct. Anal. 9 (1999), 370-392.

[Mer08] Merker, J.: Jets de Demailly-Semple d'ordres 4 et 5 en dimension 2. Int. J. Contemp. Math. Sci. 3-18 (2008), 861-933.

[Mer09] Merker, J.: Low pole order frames on vertical jets of the universal hypersurface Ann. Inst. Fourier (Grenoble), 59 (2009), 1077-1104.

[Mer10] Merker, J.:Application of computational invariant theory to Kobayashi hyperbolicity and to GreenGriffiths algebraic degeneracy J. of Symbolic Computation, 45 (2010) 9861074.

[Mer15] Merker, J.: Complex projective hypersurfaces of general type: towards a conjecture of Green and Griffiths arXiv:1005.0405; Algebraic differential equations for entire holomophic curves in projective hypersurfaces of general type: optimal lower degree bound. In: Geometry and Analysis on Manifolds, in memory of Professor Shoshichi Kobayashi, (eds. T. Ochiai, T. Mabuchi, Y. Maeda, J. Noguchi and A. Weinstein), Progress in Mathematics, Birkhäuser 308 (2015), $41-142$.

[MeTa19] Merker, J., Ta, The-Anh: Degrees $d \geqslant(\sqrt{n} \log n)^{n}$ and $d \geqslant(n \log n)^{n}$ in the Conjectures of Green-Griffiths and of Kobayashi. arXiv:1901.04042, math.AG.

[Mey89] Meyer, P.-A.: Qu'est ce qu'une différentielle d'ordre n? Expositiones Math. 7 (1989), 249-264.

[Miy82] Miyaoka, Y.: Algebraic surfaces with positive indices. Classification of algebraic and analytic manifolds. Katata Symp. Proc. 1982, Progress in Math., vol. 39, Birkhäuser, 1983, 281-301.

[MoMu82] Mori, S., Mukai, S.: The uniruledness of the moduli space of curves of genus 11. In: Algebraic Geometry Conference Tokyo-Kyoto 1982, Lecture Notes in Math. 1016, 334-353.

[Nad89] Nadel, A.M.: Hyperbolic surfaces in $\mathbb{P}^{3}$. Duke Math. J. 58 (1989), 749-771.

[Nog77a] Noguchi, J.: Holomorphic curves in algebraic varieties. Hiroshima Math. J. 7 (1977), 833-853.

[Nog77b] Noguchi, J.: Meromorphic mappings into a compact complex space. Hiroshima Math. J. 7 (1977), 411-425.

[Nog81a] Noguchi, J.: A higher-dimensional analogue of Mordell's conjecture over function fields. Math. Ann. 258 (1981), 207-212.

[Nog81b] Noguchi, J.: Lemma on logarithmic derivatives and holomorphic curves in algebraic varieties. Nagoya Math. J. 83 (1981), 213-233.

[Nog86] Noguchi, J.: Logarithmic jet spaces and extensions of de Franchis' theorem. Contributions to Several Complex Variables, Aspects of Math., E9, Vieweg, Braunschweig (1986), 227-249.

[Nog91] Noguchi, J.: Hyperbolic manifolds and diophantine geometry. Sugaku Expositiones 4, Amer. Math. Soc. Providence, RI (1991), 63-81.

[Nog96] Noguchi, J.: Chronicle of Bloch's conjecture. Private communication.

[Nog98] Noguchi, J.: On holomorphic curves in semi-abelian varieties. Math. Z. 228 (1998), 713-721.

[NoOc90] Noguchi, J.; Ochiai, T.: Geometric function theory in several complex variables. Japanese edition, Iwanami, Tokyo, 1984 ; English translation, xi + 282 p., Transl. Math. Monographs 80, Amer. Math. Soc., Providence, Rhode Island, 1990.

[NoWi13] Noguchi, J., Winkelmann, J.: Nevanlinna Theory in Several Complex Variables and Diophantine Approximation. GrundLehren der Math. Wiss. Vol. 350, Springer, Tokyo-Heidelberg-New York-Dordrecht-London, 2014.

[NWY07] Noguchi, J., Winkelmann, J., Yamanoi, K.: Degeneracy of holomorphic curves into algebraic varieties. J. Math. Pures Appl. (9) 88, No. 3, (2007), 293-306.

[NWY13] Noguchi, J., Winkelmann, J., Yamanoi, K.: Degeneracy of holomorphic curves into algebraic varieties. II. Vietnam J. Math. 41, No. 4, (2013), 519-525.

[Och77] Ochiai, T.: On holomorphic curves in algebraic varieties with ample irregularity. Invent. Math. 43 (1977), 83-96. 
[Pac04] Pacienza, G.: Subvarieties of general type on a general projective hypersurface. Trans. Amer. Math. Soc. 356 (2004), 2649-2661.

[PaRo07] Pacienza, G., Rousseau, E.: On the logarithmic Kobayashi conjecture. J. reine angew. Math. 611 (2007), 221-235.

[Pau08] Păun, M.: Vector fields on the total space of hypersurfaces in the projective space and hyperbolicity. Math. Ann. 340 (2008) 875-892.

[RiYa18] Riedl, E., Yang, D.: Applications of a grassmannian technique in hypersurfaces. June 2018, arXiv:1806.02364, math.AG.

[Rou06] Rousseau, E.: Étude des jets de Demailly-Semple en dimension 3. Ann. Inst. Fourier (Grenoble) 56 (2006), 397-421.

[Roy71] Royden, H.: Remarks on the Kobayashi metric. In: Several Complex Variables II, Proc. Maryland Conference on Several Complex Variables, Lecture Notes, Vol. 185, Springer-Verlag, Berlin (1971), 125-137.

[Roy74] Royden, H.: The extension of regular holomorphic maps. Proc. Amer. Math. Soc. 43 (1974), 306-310.

[Sei68] Seidenberg, A.: Reduction of the singularities of the differential equation Ady $=B d x$. Amer. J. of Math. 90 (1968), 248-269.

[Sem54] Semple, J.G.: Some investigations in the geometry of curves and surface elements. Proc. London Math. Soc. (3) 4 (1954), 24-49.

[ShZa02] Shiffman, B., Zaidenberg, M.: Hyperbolic hypersurfaces in in $\mathbb{P}^{n}$ of Fermat-Waring type. Proc. Amer. Math. Soc. 130 (2002), 2031-2035.

[Shi98] Shirosaki, M.: On some hypersurfaces and holomorphic mappings. Kodai Math. J. 21, no. 1, (1998), 29-34.

[Siu76] Siu, Y.T.: Every Stein subvariety admits a Stein neighborhood. Invent. Math. 38 (1976) 89-100.

[Siu87] Siu, Y.T.: Defect relations for holomorphic maps between spaces of different dimensions. Duke Math. J. 55 (1987), 213-251.

[Siu93] Siu, Y.T.: An effective Matsusaka big theorem. Ann. Inst. Fourier (Grenoble), 43 (1993), 1387-1405.

[Siu97] Siu, Y.T.: A proof of the general Schwarz lemma using the logarithmic derivative lemma. Personal communication, April 1997.

[Siu02] Siu, Y.T.: Some recent transcendental techniques in algebraic and complex geometry. Proceedings of the International Congress of Mathematicians, Vol. I (Beijing, 2002), Higher Ed. Press, Beijing (2002) 439-448.

[Siu04] Siu, Y.T.: Hyperbolicity in complex geometry. The legacy of Niels Henrik Abel, Springer, Berlin (2004) 543-566.

[Siu15] Siu, Y.T: Hyperbolicity of generic high-degree hypersurfaces in complex projective spaces. Inventiones Math. 202 (2015) 1069-1166.

[SiYe96a] Siu, Y.T., Yeung, S.K.: Hyperbolicity of the complement of a generic smooth curve of high degree in the complex projective plane. Invent. Math. 124 (1996), 573-618.

[SiYe96b] Siu, Y.T., Yeung, S.K.: A generalized Bloch's theorem and the hyperbolicity of the complement of an ample divisor in an abelian variety. Math. Ann. 306 (1996), 743-758.

[SiYe97] Siu, Y.T., Yeung, S.K.: Defects for ample divisors of Abelian varieties, Schwarz lemma and hyperbolic hypersurfaces of low degree. Amer. J. Math. 119 (1997), 1139-1172.

[Tra95] Trapani, S.: Numerical criteria for the positivity of the difference of ample divisors. Math. Z. 219 (1995), 387-401.

[Tsu88] Tsuji, H.: Stability of tangent bundles of minimal algebraic varieties. Topology 27 (1988), 429-442.

[Ven96] Venturini S.: The Kobayashi metric on complex spaces. Math. Ann. 305 (1996), 25-44.

[Voi96] Voisin, C.: On a conjecture of Clemens on rational curves on hypersurfaces. J. Diff. Geom. 44 (1996) 200-213, Correction: J. Diff. Geom. 49 (1998), 601-611.

[Voj87] Vojta, P.: Diophantine approximations and value distribution theory. Lecture Notes in Math. 1239, Springer-Verlag, Berlin, 1987.

[Win07] Winkelmann, J.: On Brody and entire curves. Bull. Soc. Math. France 135 (2007), 25-46.

[Xie15] Xie, S.-Y:: On the ampleness of the cotangent bundles of complete intersections. Inventiones Math. 212 (2018) 941-996. 
[Xu94] Xu, G.: Subvarieties of general hypersurfaces in projective space. J. Differential Geometry 39 (1994), 139-172.

[Zai87] Zaidenberg, M.G.: The complement of a generic hypersurface of degree $2 n$ in $\mathbb{C P}^{n}$ is not hyperbolic. Siberian Math. J. 28 (1987), 425-432.

[Zai93] Zaidenberg, M.G.: Hyperbolicity in projective spaces. International Symposium on Holomorphic mappings, Diophantine Geometry and Related topics, 1992, R.I.M.S. Lecture Notes ser. 819, R.I.M.S. Kyoto University (1993), 136-156.

Version of June 19, 2019, proof corrected on November 28, 2019, printed on February 14, 2020, 2:06

The original publication is available at www.springerlink. com (Japanese Journal of Mathematics)

Jean-Pierre Demailly

Université Grenoble Alpes, Institut Fourier (Mathématiques)

UMR 5582 du C.N.R.S., 100 rue des Maths, 38610 Gières, France

e-mail: jean-pierre.demailly@univ-grenoble-alpes.fr 